UNIVERSITY OF THESSALY

SCHOOL OF HEALTH SCIENCES

FACULTY OF VETERINARY SCIENCE

DEPARTMENT OF SURGERY

\title{
ULTRASONOGRAPHIC, \\ COSMETIC, CLINICAL AND HISTOLOGICAL STUDY OF THE HEALING PROCESS IN THE CANINE SKIN FOLLOWING SURGICAL INCISION AND CLOSURE USING VARIOUS TECHNIQUES
}

\author{
DIMITRIOS B. BALOMENOS \\ DVM
}

Ph.D. THESIS

KARDITSA 2018 
Institutional Repository - Library \& Information Centre - University of Thessaly 26/04/2023 13:56:30 EEST - 54.147.182.235 
UNIVERSITY OF THESSALY

SCHOOL OF HEALTH SCIENCES

FACULTY OF VETERINARY SCIENCE

DEPARTMENT OF SURGERY

\title{
ULTRASONOGRAPHIC, COSMETIC, CLINICAL AND HISTOLOGICAL STUDY OF THE HEALING PROCESS IN THE CANINE SKIN FOLLOWING SURGICAL INCISION AND CLOSURE USING VARIOUS TECHNIQUES
}

\author{
DIMITRIOS B. BALOMENOS \\ DVM
}

\section{Ph.D. THESIS}

\begin{abstract}
Examination Committee
Apostolos D. Galatos, Professor

Supervisor Jimmy Saunders, Professor

Member of the Advisory Committee

Pagona G. Gouletsou, Associate Professor

Member of the Advisory Committee Lysimachos Papazoglou, Professor Member of the Examination Committee Nikolaos Papaioannou, Professor Member of the Examination Committee Serafim Papadimitriou, Associate Professor Member of the Examination Committee Nikitas Prassinos, Associate Professor Member of the Examination Committee
\end{abstract}


Institutional Repository - Library \& Information Centre - University of Thessaly 26/04/2023 13:56:30 EEST - 54.147.182.235 


\section{To my family}


Institutional Repository - Library \& Information Centre - University of Thessaly 26/04/2023 13:56:30 EEST - 54.147.182.235 


\section{CONTENTS}

\section{PREFACE}

INTRODUCTION

\section{PART ONE}

SKIN ANATOMY OF THE DOG 3

Skin anatomy 3

Epidermis 3

Dermis or Corium 3

Hypodermis or subcutis or subcutaneous tissue $\quad 4$

Skin glands $\quad 4$

Skin muscles $\quad 4$

Vessels and nerves of the skin 4

PRINCIPLES OF PLASTIC SURGERY 5

Wound healing 5

Inflammation phase 5

$\begin{array}{ll}\text { Proliferation and repair phase } & 6\end{array}$

$\begin{array}{ll}\text { Maturation and remodeling phase } & 7\end{array}$

Complications of skin healing 8

Delayed wound healing $\quad 8$

$\begin{array}{lr}\text { Excessive scar tissue formation } & 8\end{array}$

$\begin{array}{ll}\text { Infection } & 9\end{array}$

Abscess or fistula formation $\quad 9$

Oedema 9

Wound bleeding - haematoma formation 9

Seroma formation $\quad 9$

$\begin{array}{ll}\text { Wound dehiscence } & 10\end{array}$

$\begin{array}{ll}\text { Repair by first intention- wound closure techniques } & 10\end{array}$

$\begin{array}{ll}\text { Skin closure techniques } & 10\end{array}$

$\begin{array}{ll}\text { Appositional Sutures } & 10\end{array}$

$\begin{array}{ll}\text { Intradermal suture pattern } & 11\end{array}$

$\begin{array}{lr}\text { Tissue reaction to suture materials } & 12\end{array}$

$\begin{array}{ll}\text { Suture material selection } & 12\end{array}$

$\begin{array}{ll}\text { Suture materials used for intradermal skin suturing } & 13\end{array}$

$\begin{array}{ll}\text { Skin tissue adhesives } & 14\end{array}$

$\begin{array}{lr}\text { Surgical skin staples } & 18\end{array}$

Cutaneous ultrasonography $\quad 22$

$\begin{array}{ll}\text { Principles of ultrasonography and instrumentation } & 23\end{array}$

Ultrasound appearance of normal skin anatomy and main uses $\begin{array}{ll}\text { of cutaneous ultrasonography } & 24\end{array}$

B-scan ultrasound wound area measurement of skin in the dog 26 
PART TWO

MATERIALS AND METHODS 28

Dogs 28

$\begin{array}{lr}\text { Inclusion criteria } & 28\end{array}$

Initial and continuous evaluation of health status $\quad 28$

Experimental protocol 28

$\begin{array}{ll}\text { Anaesthesia and surgical operation } & 29\end{array}$

Postoperative treatment 30

$\begin{array}{ll}\text { Evaluation of wound healing of skin incisions } & 30\end{array}$

Cosmetic evaluation $\quad 30$

Clinical evaluation 31

Ultrasonographic evaluation of the healing process 31

Histological evaluation of the healing process 32

Statistical Analysis 33

RESULTS $\quad 35$

Time required for each technique $\quad 35$

$\begin{array}{ll}\text { Cosmetic evaluation } & 35\end{array}$

Comparison of cosmetic evaluation among assessors 35

$\begin{array}{ll}\text { Total cosmetic evaluation } & 37\end{array}$

Clinical evaluation 38

Skin thickening 38

Erythema $\quad 40$

Scar width $\quad 41$

Inflammation and Abscessation $\quad 45$

Exudate 46

Comedones 46

Hyperpigmentation at the wound area $\quad 46$

Staples loss $\quad 48$

Wound dehiscence $\quad 48$

Cross scaring formation $\quad 48$

$\begin{array}{ll}\text { Total clinical evaluation } & 49\end{array}$

Ultrasonographic evaluation $\quad 50$

Histological evaluation $\quad 59$

$\begin{array}{lr}\text { Oedema } & 59\end{array}$

Inflammation $\quad 59$

$\begin{array}{ll}\text { Thickness of the epidermis } & 60\end{array}$

$\begin{array}{ll}\text { Epithelial gap } & 61\end{array}$

$\begin{array}{ll}\text { Scar width } & 62\end{array}$

Presence of suture material $\quad 63$

Tissue reaction around the suture material 63

Fibroblasts presence $\quad 64$

Collagen deposition $\quad 64$ 
Angiogenesis

$\begin{array}{ll}\text { Total histological evaluation } & 66\end{array}$

$\begin{array}{ll}\text { Histological features } & 67\end{array}$

$\begin{array}{ll}\text { Skin stapling } & 67\end{array}$

$\begin{array}{ll}\text { Skin glue } & 73\end{array}$

Intradermal suture pattern with burying of the knot 82

$\begin{array}{ll}\text { Intradermal suture pattern with clips } & 91\end{array}$

Total evaluation of each technique on the $7^{\text {th }}, 14^{\text {th }}, 28^{\text {th }}, 180^{\text {th }}$ and $365^{\text {th }}$ po.d. $\quad 96$

$\begin{array}{ll}\text { Correlations } & 99\end{array}$

Correlation between u/s wound area and hp inflammation 99

Correlation between u/s wound area and histopathologicaly scar width 99

Correlation between u/s wound area and clinical evaluated skin thickening $\quad 99$

Correlation between $\mathrm{u} / \mathrm{s}$ wound area and clinical evaluated scar width 99

$\begin{array}{lr}\text { DISCUSSION } & 99\end{array}$

Surgical operation $\quad 99$

$\begin{array}{ll}\text { Cosmetic evaluation of the incisions } & 100\end{array}$

$\begin{array}{ll}\text { Clinical evaluation } & 101\end{array}$

$\begin{array}{ll}\text { Skin thickening at the wound area } & 102\end{array}$

$\begin{array}{ll}\text { Erythema presence } & 103\end{array}$

$\begin{array}{ll}\text { Scar width } & 103\end{array}$

$\begin{array}{ll}\text { Absessation or inflammation and exudate } & 105\end{array}$

$\begin{array}{lr}\text { Comedones } & 105\end{array}$

$\begin{array}{lr}\text { Scar hyperpigmentation } & 106\end{array}$

$\begin{array}{ll}\text { Wound dehiscence } & 107\end{array}$

Cross scaring formation 107

$\begin{array}{lr}\text { Total clinical evaluation } & 108\end{array}$

$\begin{array}{ll}\text { Ultrasonographic evaluation } & 109\end{array}$

Histological evaluation $\quad 110$

$\begin{array}{ll}\text { Necrosis } & 111\end{array}$

Oedema 111

$\begin{array}{ll}\text { Inflammation } & 112\end{array}$

$\begin{array}{ll}\text { Epidermal thickness at wound area } & 114\end{array}$

$\begin{array}{ll}\text { Epithelial gap } & 115\end{array}$

$\begin{array}{ll}\text { Histological scar width } & 115\end{array}$

Suture material presence and tissue reaction around the suture material 116

Fibroblasts presence, collagen deposition and angiogenesis 117

$\begin{array}{ll}\text { Total histological evaluation } & 118\end{array}$

$\begin{array}{ll}\text { Total evaluation of each technique } & 118\end{array}$

$\begin{array}{ll}\text { Correlations } & 119\end{array}$

Correlation between u/s wound area and histologically evaluated 120

Correlation between u/s wound area and histologically evaluated scar width $\quad 120$

$\begin{array}{ll}\text { Correlation between } \mathrm{u} / \mathrm{s} \text { wound area and clinical evaluated skin thickening } & 120\end{array}$

$\begin{array}{ll}\text { Correlation between u/s wound area and clinical evaluated scar width } & 120\end{array}$ 
CONCLUSIONS 120

ABSTRACT

124

REFERENCES

128

ADDENDUM 1a

ADDENDUM $1 b$

ADDENDUM 2 


\section{PREFACE}

In the present study, the ultrasonographic, cosmetic, clinical and histological evaluation of the surgical skin wound healing in dogs - using three closure techniques and four closure materials - was investigated. The research was conducted at the Department of Surgery, Faculty of Veterinary Medicine, School of Health Sciences, University of Thessaly and was completed with the contribution of several people who I would like to thank for their valuable assistance.

First of all, my special thanks go to the members of the supervising committee for their continual guidance, patience, and faith in this demanding research project, in particular to:

Dr. A.D. Galatos, Professor, Head of the Department of Surgery, Faculty of Veterinary Medicine, School of Health Sciences, University of Thessaly, and supervisor of the Ph.D. thesis, for his collaboration, invaluable help, and support during the preparation of the thesis and especially during the final stage of writing.

Dr. P.G. Gouletsou, Associate Professor at the Department of Obstetrics and Reproduction, Faculty of Veterinary Medicine, School of Health Sciences, University of Thessaly, for her valuable assistance during the preparation of the research protocol, and her vital contribution to the experimental surgery. Furthermore, I am grateful to her for her constructive criticism and useful suggestions during the preparation of the thesis, as well as for her confidence in me from the beginning of our working relationship.

Dr. J.Saunders, Professor, Head of the Department of Medical Imaging and Orthopaedics of Small Animals, for his valuable contribution to the $\mathrm{PhD}$ thesis

In addition, my heartfelt thanks for their contribution to the study and their continuous support and cooperation go to:

Dr. V. Tsioli and Dr. A.I. Sideri, Associate and Assistant Professor, respectively, at the Department of Surgery, Faculty of Veterinary Medicine, School of Health Sciences, University of Thessaly,

Dr. A. Lymperis and Mr. G. Kaltsas, veterinarians and $\mathrm{PhD}$ holder and $\mathrm{PhD}$ candidate, respectively, at the Department of Surgery, Faculty of Veterinary Medicine, School of Health Sciences, University of Thessaly, for their friendship and constant encouragement during the conducting of the research,

Mr. A.X. Lemas, veterinarian, for his constant support, encouragement, patience, and friendship throughout the study,

Mrs. F. Rizava and Mr. A. Troukis, staff of the Department of Surgery, for their valuable assistance in the care of dogs used in the study,

Mr. P. Mantis, Senior Lecturer at the Royal Veterinary College, for his special contribution and his invaluable help and constructive suggestions regarding the interpretation of the ultrasound images

Longport INC. High-Resolution Ultrasound company, for the provision of the specialized ultrasound instrumentation to the Department of Surgery and the continuing support. 
Mr. K. Krikonis, mathematician, MSc, Chief of DatAnalysis Company, for his invaluable help in the statistical analysis of the data.

Finally, unlimited thanks go to my beloved wife, Ntina, and my children, ByronPaul and Olga, for their patience, support and love, and to my dog, Tito, for his warm companionship throughout the writing of this thesis. 


\section{INTRODUCTION}

Most surgical wounds are typically treated by first intention healing with the use of sutures; however alternative closure techniques or materials, such as tissue adhesives and skin staples have also been used. Sutures enable meticulous closure and staples and tissue adhesives are credited for faster closure. The closed edges of the wound should be held in apposition until tensile strength of the wound is sufficient to withstand stress. Most of the studies that determine the effects of various techniques used for closure of surgical wounds in humans and dogs focus on the cosmetic appearance and the patient's and/or surgeon's satisfaction (Hopkinson and Bullen, 1982, Leaper and Benson 1985, Orozco-Covarrubias and Ruiz-Maldonado 1999, Runk et al. 1999, Sylvestre et al. 2002, Ridgway et al. 2007). Traditionally, non-invasive assessment of wound healing has relied on photography. However, photography provides a visual impression of the wound at the surface level only. Surgical wound healing assessment often involves scoring of erythema and exudate or infection. The surgical scar remains the only visible evidence of the surgeon's skill and very often his efforts are judged by its final appearance. More detailed information on the state of the wound has relied on invasive skin biopsy, providing tissue samples that yield objective quantitative data relevant to the healing process but this inevitably produces further damage. Cutaneous ultrasonography is a novel technology for skin wound assessment. The conversion and recording of sound as cinematic image and its application as a method of diagnostic imaging in human and other animal species offers the possibility of monitoring and recording changes to organs and tissues (shape and position) of the body over time, and permits the evaluation, comparison and additional assistance in the clinical interpretation of findings. Cutaneous ultrasonography was first mentioned in 1979, as a modern non-invasive technique for the measurement of skin thickness by 15 $\mathrm{MHz}$ ultrasound (Alexander- Miller). Since then, the interest in the use and application of ultrasonography in the examination of the skin has grown rapidly. The development of larger ultrasonic frequencies (above $20 \mathrm{MHz}$ ) and the introduction of "ultrasound biomicroscopy" in diagnostic imaging enhanced qualitative assessment of skin composition, monitoring and evaluation of skin function in various pathological conditions, and enabled assessment of small open skin wounds which heal by second intention (Mantis et al. 2005, 2007). By measuring the area of the skin wound the process and progression of open wound healing can be assessed without interfering with healing. However, up to date, ultrasonography has not been used as a means of studying the healing process of wounds or surgical incisions closed by different methods and materials neither in humans nor in other animal species.

The aims of the present study was to compare the efficacy of various techniques used for the closure of skin incisions in dogs via ultrasonographic (50 MHz), cosmetic, clinical and histological evaluation, to describe the ultrasonographic appearance of the healing skin, and to compare it with clinical and histological measurements. Whether the findings, obtained when very high frequencies are used, actually represent a valid adjunct to clinical and histological assessment of the healing process of the skin was 
also investigated. Furthermore, the difficulty and the time required to close each wound was also estimated. The following techniques and materials for wound closure were compared: (a) Skin staples, (b) n-butyl cyanoacrylate tissue glue, (c) Continuous intradermal suture pattern (with burying of the knot) by using 4-0 poliglecaprone 25 and (d) Continuous intradermal suture pattern by using polypropylene 4-0 with attachment clips (without burying the knot).

In the first part of this thesis, the essential elements of the skin's anatomy and the principles of plastic surgery and of ultrasonography are presented. In the second part of the thesis, the materials and techniques used in the present study are described in detail, followed by the statistical analysis of the data of the present study, and the results of the study are discussed in association with the current literature. The thesis ends with the conclusions of the study, an abstract, the list of references and addendum with the photos of skin wounds and ultrasonograms. 


\section{PART ONE}

\section{SKIN ANATOMY}

The skin or integument is the largest organ of the body and in mammals consists of the epidermis and the dermis or corium. Subcutaneous tissue or hypodermis or subcutis (typically described with the skin), is not part of the skin but a superficial layer (Monteiro-Riviere et al 1993), which contributes to the connection of the skin to the underlying tissues (Banks 1993, Monteiro-Riviere et al 1993).

\section{Epidermis}

The epidermis is the outer layer of the skin and consists of multilayer squamous keratinized epithelium, which is continuously subjected to proliferation procedures, differentiation, and keratinization (Monteiro-Riviere et al 1993). The number of its layers varies depending on body area (Banks 1993, McGeady et al 2006b). The layers of the skin, from inside out, are stratum basale, stratum spinosum, stratum granulosum, stratum lucidum and the stratum corneum (Monteiro-Riviere et al 1993).

The epithelial cells of stratum basale have a continuous mitotic activity which plays a role in the renewal (or regeneration) of the epidermal epithelial cells. Melanocytes and cells of Merkel are situated in this layer. The first is responsible for skin pigmentation and for ultraviolet radiation protection. (Banks 1993, Monteiro-Riviere et

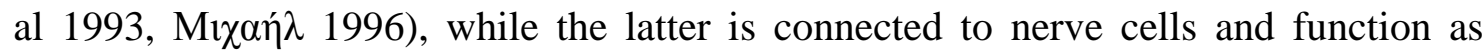
mechanoreceptors of touch (Banks 1993, Monteiro-Riviere et al 1993, Scott et al 2001a).The stratum spinosum is thicker on paw pads, nasal planum, and mucocutaneous areas compared to other areas of the body. Langerhans cells are located in this layer and are thought to be involved in skin immune reactions (Monteiro-Riviere

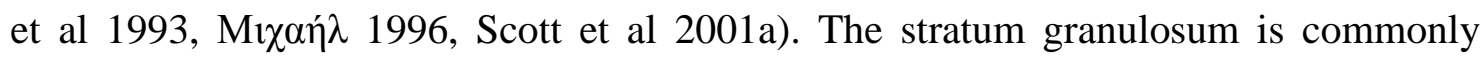
found in hairy skin areas and is highly developed in paw pads (Monteiro-Riviere et al 1993, Scott et al 2001a). The stratum lucidum exists only in areas of the body where the skin is thick, e.g. the palmar and plantar surfaces and the nasal planum (MonteiroRiviere et al 1993, Scott et al 2001a). The stratum corneum consists of many layers of fully keratinized epithelial cells. At the most superficial layer, cells normally form thin and free keratin flakes which compose the stratum disjuctum. (Monteiro-Riviere et al

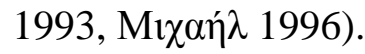

\section{Dermis or corium}

The dermis is located internally of the epidermis, from which it is separated by the basement membrane, and extends to the subcutis. It also contributes to the structure and function of the epidermis and plays an important role in the reconstruction of the skin (Scott et al 2001a). The dermis is mainly composed of dense connective tissue and collagen, elastic and reticular fibers (McGeady et al 2006b). Dermis cells are mainly fibroblasts, mast cells, and macrophages, and often include plasmocytes, fat cells, and 
extravascular leukocytes. Skin blood vessels, lymphatics, nerves, sebaceous and sweat glands and the hair follicles and the hair retractor muscles are found in the dermis (Banks 1993, Monteiro-Riviere et al 1993, Mıфй́ 1996).

\section{Hypodermis or subcutis or subcutaneous tissue}

The subcutaneous tissue makes the skin flexible and more elastic in relation to the underlying tissues, due to its content of loose connective tissue and collagen and elastic fibers. Furthermore, it contains fat cells, the number of which varies depending on the area of the body and the nutritional status of the animal, which form the subcutaneous fat (panniculus adiposus). Finally, in the subcutaneous tissue, there are blood vessels, lymph vessels, nerves and various receptors (Banks 1993, Monteiro-Riviere et al 1993,

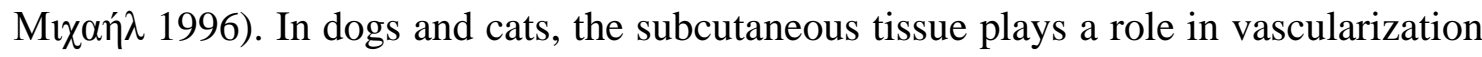
and skin healing. (Bohling et al 2006).

\section{Skin glands}

The glands of the skin are divided into sebaceous and sweat glands. In domestic mammals, the sebaceous glands are located throughout the skin except in the keratinized skin of nasal planum and paw pads (Banks 1993, Monteiro-Riviere et al

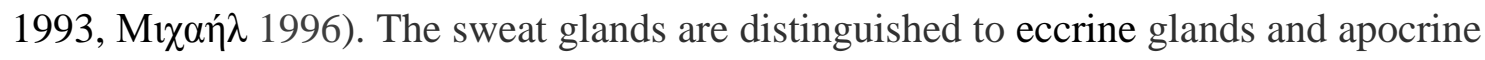
glands according to their morphological and functional characteristics. Eccrine sweat

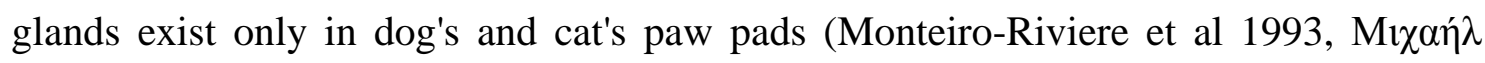
1996). Apocrine sweat glands are the main type of sweat glands in domestic mammals and are distributed almost all over the body. However, these glands' secretion is not

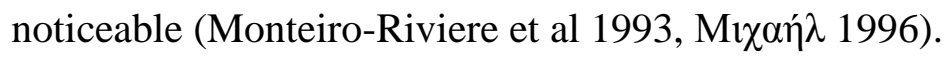

\section{Skin muscles}

Skin muscles are the panniculus muscle or panniculus carnosus and the hair retractor muscles. In dogs, the panniculus muscle consists of minute cutaneous muscles located under the skin throughout most of the body. This muscle is located under the dermis, is connected to it and contributes to the overlying skin movement and vasculature (Pavletic 2010).

\section{Vessels and nerves of the skin}

The vessels of the skin form three interconnected plexi of arteries and veins: the deep, subdermal or subcutaneous, the middle or cutaneous and superficial or subpapillary plexus. The deep vascular plexus is the major skin vascular network. The course vessels extend more superficially in the dermis, giving branches from which the middle vascular plexus is formed. In the superficial dermis, the superficial vascular plexus consists of a middle plexus branche (Pavletic 2010).

Skin lymphatic vessels derive from capillary networks located in the superficial dermis and drain into the subcutaneous lymphatic vessels matrix (Scott et al 2001a).

The skin nervous plexus derives from small subcutis nerves and the nervous plexus fibers which penetrate the dermis and provide epidermic nervous endings (MonteiroRiviere et al 1993, Scott et al 2001a). 


\section{PRINCIPLES OF PLASTIC SURGERY}

\section{Wound Healing}

Wound healing is a complex normal physiological process that restores the continuity of tissues after an injury, characterized by biochemical, microscopic and macroscopic events and characteristics (Hosgood 2009).

Although regeneration, i.e. the formation of tissue with a similar structure and comparable functions to intact skin, is uncommon (with notable exceptions such as early fetal healing and partial thickness epidermal wounds), healing does result in a structurally and functionally satisfactory but not identical outcome ( $\mathrm{Li}$ et al 2007). Generally, in total thickness skin wound healing, only the epidermis regenerates, while dermis with the glands and hair follicles are replenished from scar tissue by repair. In contrast, subcutaneous tissue is repaired and is usually accompanied by normal appearance and function (Johnston 1990). Two different modes of wound repair may be distinguished, relating mainly to surgical approximation or not of the wound edges. When repair by first intention is performed, wound edges are approximated directly by using a wound closure technique. When repair by second intention is chosen, the edges of the wound are not approximated but are allowed to heal through contraction and epithelialization of the wound. Repair by first intention is always faster than repair by second intention (Johnston 1990).

The overlapping segments of the repair process are conceptually defined as inflammation, proliferation, and remodeling. During the inflammatory phase, hemostasis occurs and an acute inflammatory infiltrate ensues. The proliferative phase is characterized by angiogenesis, fibroplasia, contraction, and epithelialization. The final phase is remodeling, which is commonly described as scar maturation (Peter Lorenz and Longaker 2008).

\section{Inflammation Phase}

The inflammatory phase begins immediately after the injury, lasts for four or five days and is marked by a hypoxic, ischemic environment (Wysocki 1989, Greenberg and Clark 2009). Vasoconstriction and haemostasis, vasodilatation and increased capillary permeability, secretion of chemotactic growth factors and cytokines, and phagocytosis constitute the main processes that take place during this phase, leading to an influx of white blood cells (neutrophils, monocytes, macrophages, lymphocytes), connective tissue cells and endothelial cells to the wound site, responsible for wound debridement, fibroplasia stimulation and subsequent collagen production. Blood clotting within the vessel lumen reestablishes haemostasis, whereas the extravascular clot dries and forms an eschar or scab that stabilizes wound edges, protects the wound from infection and fluid loss, and later provides a provisional matrix or scaffold for cellular migration as substrata for the early reorganization of the wound (Pavletic 2010). This stage is easily recognized by the four cardinal signs of inflammation: redness, heat, pain, and swelling (Wysocki 1989). During inflammation, the biomechanical strength of the wound is still weak, and most of this strength is provided by fibrin within the blood clot (Hosgood 2003). 


\section{Proliferation and Repair Phase}

The proliferation and repair phase usually begins three to five days after injury and lasts up to twenty days after injury. Angiogenesis, fibroplasia, and epithelialization are all proliferative processes that occur during this phase. Wound contraction also occurs during this phase (Hedlund 2007, Hosgood 2009).

New capillaries develop from pre-existing vessels at the wound edges and from endothelial cells (proliferation) which have migrated into the provisional extracellular matrix. Capillary buds extend into the matrix, where their tips eventually branch and connect, forming capillary loops. New branches extend from the loops to form a capillary plexus. Angiogenesis is stimulated by factors produced by macrophages, platelets and endothelial cells and local wound changes such as low oxygen tension, increased lactate and low pH (Hosgood 2009, Pavletic 2010).

At the same time that endothelial cells are entering the wound, populations of mesenchymatic cells that are fibroblastic, also move into the wound through the filamentous fibrin-reach provisional extracellular matrix for dermal reconstitution (Hosgood 2003, Li et al 2007). Fibroblasts originate from the viable "peri-wound" tissues, although other viable mesenchymal cells are also capable of differentiating into fibroblasts (Pavletic 2010). After migration and proliferation into the wound, fibroblasts synthesize proteins and the provisional extracellular matrix is gradually replaced by a collagenous extracellular matrix including collagens, proteoglycans, and elastin, and progressively reorganized into a durable connective tissue (Pavletic 2010). The greatest rate of accumulation of connective tissue occurs at the wound site 7-14 days after injury, corresponding to the rapid accumulation of collagen in the wound and the rapid gain in wound strength. The combination of new capillaries, fibroblasts, and fibrous connective tissue forms the characteristic red fleshy granulation tissue that fills and protects the wound, provides a barrier to infection, and promotes wound epithelialization and contraction The first interstitial collagen present is type III, but as the wound matures, type I collagen overtakes the type III collagen (Hosgood 2009). Collagen is directly responsible for the tensile strength of a healing wound. Days 4-5 is the time at which fibroplasia and early collagen deposits are noted, and net collagen synthesis is increased for at least 4-5 weeks after wounding, spanning the proliferative and maturation phases of healing. The termination of this phase is noted by a decline in the number of capillaries and fibroblasts and by a greater deposit of collagen (Pavletic 2010).

The predominant early activity in epithelialization is mobilization and migration of epidermal cells at the margins of the wound, followed by proliferation of epidermal cells behind these leading cells 1-2 days after injury. The migrating epidermal cells move under the eschar, separating it from the wound surface (Hosgood 2003). When migration ceases, possibly as a result of contact inhibition, keratinocytes reattach themselves to the underlying substratum, reconstitute the basement membrane, and then resume the process of terminal differentiation to generate a stratified epidermis (Li et al 2007). In sutured wounds, the epidermal edges invert into the dermal portion of the incision and the epidermal cells bridge the small gap within 48 hours. In moderate to large wounds, epithelial migration may take weeks or may never 
completely cover the open wound (Pavletic 2010). Melanocytes in the skin adjacent to the wound will undergo mitosis and migrate into the regenerating epidermis. Repigmentation is progressive from the periphery of the wound to the center and there is a lag of 1-2 weeks before pigmentation is noted. Maximal melanocyte proliferation may not be seen for several months (Hedlund 2007).

\section{Maturation and Remodeling phase}

The maturation and remodeling phase is generally considered to take place from day twenty to approximately 1 year after injury. The final part of the wound healing phase is marked by the evolution of the matrix into a highly refined and ordered collagen complex. Through the introduction of contractile forces of myofibroblasts, wound contraction decreases the size of the wound and minimizes the amount of collagen deposits required, while collagen remodeling occurs, with a balance between collagen deposits and collagen catabolism, type I collagen increases in larger collagen bundles with intermolecular cross-links with adjacent collagen fibers, taking on specific orientation along the lines of tension (Greenberg and Clark 2009, Pavletic 2010). This is manifested as increasing tensile strength of the wound over the postoperative period. In the end, there is less collagen than initially noted in the early healing process but it has a structurally superior configuration (Pavletic 2010). The collagen that is initially laid down is thinner than collagen in the uninjured skin and is oriented parallel to the skin. The collagen in the scar (even after a year of maturation) will never become as organized as the collagen found in uninjured skin (Broughton II et al 2006). The final result of tissue repair is a scar, which is brittle, less elastic than normal skin, and does not contain any skin appendages such as hair follicles or sweat glands (Peter Lorenz and Longaker 2008). During remodeling, wounds gradually become stronger. At the $1^{\text {st }}$ week, the wound has only $3 \%$ of its final strength. Three weeks after injury, the scar has $20 \%$ of its final strength. Thereafter, gains of tensile strength occur at a much slower rate. Over the next several weeks, a scar will achieve only $80 \%$ of the tensile strength of normal skin and never attain the same tensile strength of normal tissue (Gregory 1999).

These phases can be allowed to proceed uninterrupted, as in healing by second intention, or influenced by primary closure with sutures, staples, or adhesives (Rivera and Spencer 2007). In sutured skin wounds, the healing process is faster than in a wound healing by second intention, if aseptic techniques, gentle tissue manipulation (handling), adequate hemostasis and materials with biological inertness are used. Specifically, the inflammation phase is very quick and the repair phase is completed within the first two postoperative days. The keratinization of the superficial layers of cells is evident on the $5^{\text {th }}$ postoperative day. The reparation of the underlying epithelial tissue begins during the third or fourth day with the increased activity of fibroblasts and new capillaries. The time of formation, deposition, modification, and orientation of collagen fibers is shorter and the tensile strength of the skin is at a satisfactory level between the $4^{\text {th }}$ and $6^{\text {th }}$ day. However, in general, tensile strength remains at levels mentioned above (Johnston 1990, Pope 1993). 
The complexity of wound healing makes it vulnerable to interruption at many levels. Factors that affect physiological responses and cellular function may influence wound healing (Broughton II et al 2006). Inadequate nutritional support, both in energy and essential amino acids, vitamins (C, B12, B6, A, E) and minerals (zinc), may delay wound healing and increase infection risk. Additionally, several drug substances can cause anorexia or gastrointestinal disturbance, leading also to inadequate food intake, while others, such as corticosteroids, adversely affect the initial phase of healing and increase the infection possibilities due to immunosuppression. Furthermore, hypovolemia, dehydration and severe anemia have negative effects on healing as do the presence of necrotic tissues and foreign bodies, violent surgical manipulations, improper post-operative treatment, systemic diseases etc (Hosgood 2003).

\section{Complications of skin healing}

The most important complications of skin healing include delayed wound healing, excessive scar tissue formation, abscess or fistula formation, and functional limitation of a body region, e.g. a limb, due to excessive wound contraction. Furthermore, in sutured surgical or other wounds, seroma formation or wound dehiscence can be observed (Pavletic 2010).

\section{Delayed wound healing}

This term is used to describe wounds that fail to heal in a timely fashion (Pavletic 2010). Apart from the factors mentioned above, other factors that may affect the completion of healing phases are: failure of the surgeon to adhere to the basic surgical principles (minimal surgical trauma, adequate hemostasis, adequate perfusion, aseptic surgical technique, avoidance of wound tension during suturing, atraumatic wound edges apposition, dead space elimination), failure to manage successfully the wound (to eliminate harmful factors, to permit tissue resting and adequate wound drainage and debridement, to prevent blood venous stasis), and presence of necrotic tissue (Pope 1993, Remedios 1999).

\section{Excessive scar tissue formation}

Formation of scar tissue is a normal response to wound healing. During wound healing, excessive scar tissue formation is undesirable, because it causes functional problems and worsens the cosmetic outcome. Atraumatic surgical techniques minimize the formation of scar tissue. In many tension wounds with initially thin scars, scar tissue may extend afterwards during maturation of the wound. Reduced tension can be achieved by suturing of subcutaneous tissue, using special skin suturing techniques, changing the angle of the incision and applying wound dressings. Suturing techniques and suture material play a significant role in the formation and final appearance of scar tissue. The use of sutures of smaller diameter permits the implantation of more sutures and a better wound edge approximation (Pavletic 2010). 


\section{Infection}

Wound bacterial infection depends on many factors, i.e. condition of the wound at the time of suturing, the extent of the wound, handling, operation timing, surgeon's experience, and pre-, intra- or postoperative administration of antibiotics (Remedios 1999). The resistance to infection and the promotion of healing critically depend upon maintaining adequate blood perfusion in the wound's tissues. The delivery of antibiotics also depends on maintaining adequate blood perfusion in the compromised tissues. The mere presence of bacteria is less important than the level of bacterial growth. Bacterial growth greater than 100,000 microorganisms per gram of tissue is necessary to cause wound infection (Pavletic 2010). Increased risk of infection is usually observed in obese or geriatric patients and patients with endocrine disorders or inflammations in body-areas other than the wound area. In a retrospective study in dogs and cats, after clean wounds suturing by experienced surgeons, without administration of antibiotics, the infection rate was $1.6 \%$ (Vasseur et al 1988). Bacterial infection results in the release of toxins and of associated inflammatory infiltrates that can result in cell death and vascular thrombosis (Pope 1995, Pavletic 2010).

\section{Abscess or fistula formation}

Abscess or fistula formation is common in dogs and cats after skin injury as a result of bites. The microorganisms that are usually isolated are Staphylococcus spp, Streptococcus spp, Pseudomonas spp., Proteus spp. and Pasteurella spp., while nocardiosis, actinomycosis, and other atypical mycobacterial infections are rarely observed. An abscess is usually formed in the inflammation area, which may later become a fistula (Pavletic 2010).

\section{Oedema}

Oedema is the accumulation of fluid in the interstitial space It is usually the result of damage to the vascular or lymphatic vessels due to trauma or surgery and may cause wound dehiscence due to a mechanical effect on the wound (Friend 2009).

\section{Wound bleeding - haematoma formation}

Intra- or postoperative wound bleeding can have a negative effect on wound healing, by causing mechanical separation of wound edges, while haematoma formation causes mechanical inhibition of neoangiogenesis and macrophage infiltration. Furthermore, haematoma constitutes a nutritious substrate for the growth of microorganisms in wound site (Friend 2009).

\section{Seroma formation}

A seroma is an accumulation of sterile fluid underneath a wound presenting as a soft swelling, non-painful on palpation that usually develops 2-5 days after surgery. Seroma can be caused by excessive "dead space" during surgery as a result of excessive tissue trauma, by leakage from capillaries or lymphatics, by traumatic surgical technique and increased mobility in the region and secondarily due to inflammation (Remedios 1999, 
Friend 2009). A seroma does not always have a catastrophic effect on the wound; however, it is desirable to avoid it because phagocytic cells find it harder to function, the fluid provides a nutrient source for bacteria and increased pressure because of the fluid causes an increase in wound bursting tension and the potential for wound dehisce (Pavletic 2010).

\section{Wound dehiscence}

Wound dehiscence is the most common complication after wound suturing and the result of a combination of several factors. Most often it is the result of the premature removal of sutures by the animal or a consequence of self-harm of the suturing area.

Wound dehiscence may be the result of an inappropriate technique of suturing. Sutures tied too tightly can compromise cutaneous circulation and, as a result, may cause tissue necrosis, suture material removal, and wound dehiscence. Suture cut - out can occur from placement too close to the incision. Because collagenase activity remains high within $5 \mathrm{~mm}$ of the skin incision, sutures placed near or within this zone are more likely to cut through the weakened dermal collagen weave, especially in the presence of motion and incisional tension

Furthermore, the cutting needles may tear the tissue at the wound edges and cause suture material removal. The use of inadequate suture material and inappropriate skin closure technique relative to the thickness, durability, tension, and mobility of the sutured skin can also be a cause of wound dehiscence (Pavletic 2010).

Inflammation and tissue necrosis or foreign body presence increases the risk of wound dehiscence, as does the closure of severely compromised skin, with subsequent necrosis. Furthermore, malnutrition, inadequate perfusion of wound, moisture accumulation contributing to tissue overhydration and maceration, absence of postoperative protection from increased mobility, licking and external injury, premature removal of suture material and suture material placement into scar tissue, which has a reduced supportive capacity, increase the risk of dehiscence (Pavletic 2010).

Finally, wound dehiscence may be the result of increased tension in the suture line, of wound oedema, seroma or hematoma formation (Friend 2009).

\section{Repair by first intention - wound closure techniques Skin closure techniques}

Ideally, the selection of wound closure technique should be based on the biologic interaction of the materials employed, tissue configuration, and biomechanical properties of the wound. The tissue should be held in apposition until tensile strength of the wound is sufficient to withstand stress. Appositional sutures, tapes, staples, and tissue adhesives are several different methods used to provide an accurate and secure approximation of the skin edges (Edlich et al 2010).

\section{Appositional sutures}

There are two main techniques for sutural closure of skin: percutaneous and dermal. Percutaneous sutures are passed through the epidermal and dermal layers of skin; synthetic nonabsorbable suture materials are preferred because they have the least 
effect on the wound defenses. Dermal or intradermal sutures reapproximate the divided edges of the dermis; either synthetic absorbable or nonabsorbable (removable) suture materials are preferred for this technique. Either technique can be used as a continuous (“running”) suture or as an interrupted suture (Edlich et al 2010).

\section{Intradermal suture pattern}

The continuous intradermal suture pattern is a plastic skin closure technique and is used for minimal scar production in wound closure. It ensures better wound edge apposition, promotes epithelialization and helps avoid wound site infections, skin irritation or track infection because the suture material is not passed through the skin into deeper tissues, (Kirpensteijn et al 1997, Trout 2003). Its disadvantages are the potentially disastrous effects of suture breakage resulting in wound dehiscence, technical difficulty and increased wound tissue handling during implementation (Smeak 1992, Trout 2009).

This skin closure technique reapproximates the divided edges of the dermis and ensures a uniform wound closure. Sutures are placed in the dermis or in areas where the subcutaneous tissue blends with the dermis. Suture material should not penetrate the epidermis because it causes foreign body tissue reaction and impairs scar tissue appearance. Either synthetic absorbable or nonabsorbable suture materials are preferred for this technique.

When synthetic absorbable suture material is used, the initial knot is buried in the subcutaneous tissue on one side of the incision and then the suture material is directed more superficially in the middle of the dermis. Then it passes horizontally from one side of the wound edge to the other, at the same depth of the dermis, thus ensuring uniform closure of the wound on a vertical plane. Carefully placed final bites are required for correct apposition and complete knot burying. The last superficial suture is left loose and then is directed back, deep into the subcutaneous tissue, where the final knot is buried while the suture material is tightened (Trout 2003). Suture material need not be removed and the implementation causes less pain (Smeac et al 1988). The buried continuous intradermal suture pattern requires substantial implanted suture material, while no suture material is protruding from the sutured wound, reducing the risk of suture material and subsequent wound contamination, and avoiding wound dehiscence caused from the animal by wound-licking or scratching (Kirpensteijn et al 1997, Trout 2003). The buried knot continuous intradermal suture pattern has been used in veterinary medicine as an alternative to the interrupted suture pattern for skin closure (Smeak 1992).

When synthetic nonabsorbable suture material is used, the suture material is anchored just before and after the commissure of the wound with 2 fixation clips or sterile buttons (with split shots) or superficial suture material loops. At the start and at the end of the wound, the needle is passed through the full skin thickness. No knots are buried in the subcutaneous tissue, thus reducing the amount of foreign material left in the wound site. The suture material is removed after several days. Non-buried knot continuous intradermal suture pattern can also be used to apply gradual tension to wound edges that cannot be initially closed (adjustable horizontal mattress suture as 
tension suture pattern) and the suture material can be periodically tightened or loosened without new suture placement (Johnson et al 1997, Trout 2003, Papazoglou et al 2010).

\section{Tissue reaction to suture materials}

The presence of foreign bodies, such as the suture material, in wounds, induces excessive inflammatory tissue responses that lower the body's defense mechanisms against infection, interfere with the proliferative phase of wound healing, and ultimately lead to inferior wound strength due to the formation of excessive scar tissue. Although normal wound healing following surgical trauma involves an inflammatory process, as briefly described previously, these reactions typically subside within a week as the inflammatory phase moves into the proliferative and repair phase. However, inflammatory tissue reactions due to the presence of suture material will persist as long as the foreign body remains within the tissue. The degree of tissue reaction, in turn, depends largely on the chemical nature and physical characteristics of the various suture materials (Greenberg and Clark 2009).

Tissue responses to implant materials occur either adjacent to the implanted material or as a systemic effect in which breakdown products of the material affect remote tissues. Four types of responses may be observed: 1) a minimal response, in which a thin layer of fibrous tissue forms adjacent to the implant, 2) a chemically induced response, in which an acute, mild inflammatory response occurs or a chronic inflammatory response develops, 3) a physically induced response, such as an inflammatory response to particulate material or to implant movement in conjunction with shape or surface roughness, and 4) necrosis (Roush 2003).

There are two distinct mechanisms of tissue reaction to foreign substances. Insoluble substances, such as metal, release corrosion products through various means, and these products are phagocytosed by either macrophages or multinucleated giant cells, depending on their size. Synthetic absorbable materials, including poliglecaprone 25, are degraded through nonenzymatic hydrolysis of ester bonds that occurs independently of inflammation. After hydrolysis, and depending on particle size, phagocytosis may take place. Absorbable synthetic suture materials gradually lose tensile strength and are absorbed in varying rates, depending on the size and composition of the suture (Roush 2003).

\section{Suture material selection}

The goal of suture material selection is to provide secure wound closure while minimizing morbidity. Suture materials are chosen on the basis of suture characteristics (initial tensile strength, relative knot security, and handling characteristics) and suture-tissue interaction (tissue reactivity, the rate of loss of tensile strength, and degradation mechanism). Although there is generally not an ideal suture material for every possible indication, certain suture materials are superior to others in different wound environments (Boothe 2003).

Certain principles are considered when selecting a suture material. Suture materials should be at least as strong as the normal tissue through which they are placed. As 
suture materials potentially cause the development of wound infection, the ideal would be that the suture material was removed or absorbed, when the sutured tissue healed.

The relative rates at which the suture loses strength and the wound gains strength are important and should be taken into consideration in suture material selection. Skin heals slowly and has been reported to achieve $20 \%$ and $42 \%$ of the normal skin's strength, 21 days and 60 days, respectively, after suturing (Booth 1998). As well as skin percutaneous suture materials are removed 10-14 days after implementation, most of the synthetic suture materials can be used for skin suturing, as they continue to keep their strength for more than 10 days. Another characteristic of the suture material is whether it is multifilament or monofilament. Multifilament suture materials are easier to handle (more pliable, without memory, making secure knots easier), but are not used for skin closure due to increased friction and subsequent injury during their passage through the tissues (Kowalsky et al 2008), and the dead space between their filaments into which microorganisms can move and grow (Bucknall 1983). Monofilament, nonabsorbable suture materials are commonly used for percutaneous suture patterns and removable intradermal suture patterns, whilst multifilament absorbable sutures are used for buried knot intradermal suture patterns.

\section{Suture materials used for intradermal skin suturing}

Poliglecaprone 25 (Monocryl ${ }^{\circledR}$, Ethilon) is an absorbable, synthetic, monofilament suture material, consisting of co-polymerization glycolic acid and caprolactone, and has excellent characteristics in handling and knot security (Roush 2003). It has high initial tensile strength, excellent pliability, and relatively rapid loss of tensile strength. It loses approximately $75 \%$ of its initial tensile strength at 14 days and essentially $100 \%$ at 21 days. It is completely absorbed within 4 months of implantation. Poliglecaprone 25 causes minimal tissue reaction, which is mainly characterized by the presence of macrophages, fibroblasts, and few neutrophils, and is inert (Boothe 2003), has no capillary action, and a low coefficient of friction (Bezwada et al 1995).

Polypropylene is a synthetic (polymer of propylene), non-absorbable, monofilament suture material (in either blue or fluorescent pink color). Polypropylene retains its strength after implantation as it is not weakened by tissue enzymes. Polypropylene is suitable for suturing tissues such as skin and cardiac muscle as it has great elongation capability. It has a relatively lower tensile strength than nylon, and relatively high knot strength and greater knot security than all the other monofilament non-metallic materials. It is resistant to bacterial contamination, inert and rather difficult to handle because it has a tendency to be stiff and has material memory. Polypropylene suture material produces minimal cellular reaction (Boothe 2003). As it has an extremely smooth surface and a very low coefficient of friction and slides easily even after 1 or 2 weeks in tissue, it is especially ideal for use in a running intradermal suture pattern (Bennett 1988).

\section{Clinical trials-findings}

Kirpensteinjn et al (1997) did not find any differences in clinical and histopathological evaluation when they compared poliglecaprone 25 with polyglactin 910 suture 
materials for skin suturing with intradermal technique in dogs. Sylvestre et al (2002) compared for 14 days the clinical and cosmetic outcome of skin incisions sutured with either poliglecaprone 25 with intradermal and simple interrupted suture patterns or polypropylene with simple interrupted suture pattern and observed that the intradermal suture pattern with poliglecaprone 25 had a superior outcome. Gouletsou (2014) compared the cosmetic, clinical and histological evaluation after suturing skin incisions with simple interrupted suture pattern and intradermal suture pattern with burying of the knots, by using two different suture materials (poliglecaprone 25 and polyglytone 6211) and two different suture diameters (3-0 and 4-0) until the $1095^{\text {th }}$ postoperative day, and found that the continuous intradermal suture pattern with burying of the knots performed with $4 / 0$ poliglecaprone 25 suture achieved a better cosmetic outcome; furthermore the intradermal suture patterns using 4-0 suture materials (polyglecaprone 25 and polyglytone 6211) were clinically evaluated as the best from the third month until the $1095^{\text {th }}$ post-operative.day. In rats, van Heerden (2005) compared with histopathological evaluation until $10^{\text {th }}$ post day poliglecaprone 25 with polyglitone 6211 in intradermal suture pattern and observed no differences. In humans, no difference was observed during cosmetic evaluation when poliglecaprone 25 was used for skin closure compared to staples until the $3^{\text {rd }}$ postoperative month (Obermair et al 2007), to polypropylene until the $4^{\text {th }}$ postoperative month (Rosenzweig et al 2010) and to skin staples until the $6^{\text {th }}$ postoperative month (de Graaf et al 2012).

Polypropylene sutures have been used for an intradermal pattern without burying the knots in cats by Papazoglou et al (2010), and the macroscopic and histologic appearance of the incisions was compared to that of the intradermal with the use of glycolide, $\varepsilon$-caprolactone, and trimethylene-carbonate suture until the $9^{\text {th }}$ postoperative day. They found no significant difference in gross evaluation scores between the closure techniques and incomplete epithelialization with glycolide, $\varepsilon$-caprolactone, and trimethylene-carbonate suture only. In humans, Vipond and Higgins (1991) did not find differences in the clinical outcome between polypropylene and polydioxanone suture until the $3^{\text {rd }}$ postoperative month and Alam et al (2006) observed better cosmetic outcome with polyglactin 910 in comparison to propylene suture until the $9^{\text {th }}$ postoperative month.

\section{Skin tissue adhesives}

In the past, the options for wound closure had been largely limited to sutures and staples, with other alternatives such as tissue adhesives having entered clinical practice more recently (Coulthard et al 2002). Tissue adhesives have been used in various forms for more than 35 years since the first cyanoacrylate adhesives were synthesized in 1949 (Cooper et al 1959). Further development led to the introduction of the cyanoacrylate derivatives that were purer and stronger, but limitations of low tensile strength and brittleness prevented widespread acceptance (Quinn et al 1993, Bruns et al 1996). More recently tissue adhesives with further improved strength have been developed, combining plasticisers and stabilizers to increase flexibility (Quinn et al 1997). 
Tissue adhesives have been used primarily in emergency rooms and in the literature is increasing support for their effectiveness in the closure of various traumatic lacerations (Quinn et al 1997, Osmond 1999, Perron et al 2000, Farion et al 2001). Surgeons have begun to use tissue adhesives in the operating room for the closure of surgical skin incisions although there are few clinical trials to support this indication (Coulthard et al 2002).

\section{Cyanoacrylates}

Cyanoacrylate tissue adhesives are currently the principal synthetic polymer sealants in clinical usage (Bhatia 2010). Cyanoacrylates were first synthesized by Ardis in 1949 (Ronis et al 1984). However, it was not until 1959 when Cooper discovered their adhesive properties that an interest arose in using these substances in surgical procedures (Ronis et al 1984). In 1966, Bhaskar et al. (1967) showed that the cyanoacrylate $(\mathrm{CH} 2=\mathrm{C}(\mathrm{CN})-\mathrm{COOR})$ chemical adhesive adheres to moist tissues, presents haemostatic properties, and is phagocytized by histiocytes. The authors carried out tests with several types of cyanoacrylates, such as ethyl cyanoacrylate, methyl cyanoacrylate, propyl cyanoacrylate, isobutyl cyanoacrylate, and butyl cyanoacrylate, with the last one showing better clinical results, followed by ethyl cyanoacrylate (Lins et al 2012). In veterinary medicine, cyanoacrylates such as N-butyl and isobutyl-2-cyanoacrylate are commonly used during some surgical procedures, such as dec1awing, tail docking, and ear cropping. Products advocated for use in veterinary patients include Tissue glue, Vetbond, and Nexabond (MacPhail 2014).

\section{Application}

Before applying a tissue adhesive, the wound should be cleaned and the bleeding should be controlled. However, absolute dryness is not required (MacPhail (2007, 2014). Cyanoacrylates should only be used for superficial skin closure and not deposited below the epidermis. When implanted subcutaneously, patients may present with signs consistent with chronic inflammation including oedema, erythema, pain, or purulent drainage. Successful use of cyanoacrylates for wound repair relies on manual skill and meticulous wound closure technique. The wound edges must be held in apposition as the adhesive is applied not only to promote eversion of the margins but also to prevent deposition of the cyanoacrylate polymer into the wound. Optimal cosmetic results will be best obtained when wound edges are well apposed with proper eversion (Mobley et al 2002).The short-chain butyl-cyanoacrylate adhesives polymerize rapidly and should be applied to the apposed wound edges only as a thin, single layer, either as discrete beads or as a continuous layer and never build up multiple layers. The high viscosity octylcyanoacrylate needs an initiator and is applied in 2 thin continuous layers, allowing 30 seconds to polymerize between layers. Care should be taken to avoid pooling or thick application of the adhesive to prevent excessive release of heat, which may cause patient discomfort and has the potential for thermal injury. No further dressings are required, no liquids or ointments, such as topical antibiotics, should be applied as they may loosen the adhesive film (Singer and Thode 2004, Singer et al 2008). 
After application, tissue adhesives rapidly polymerize in the presence of moisture and produce a strong, flexible bond. Adhesion of the contacted tissue generally takes less than a minute but may be prolonged by excessive haemorrhage (MacPhail (2014) 2007). Cyanoacrylate glues achieve adhesion through two independent mechanisms: molecular interaction via covalent bonding to the tissue surface and mechanical interlocking of the poly(cyanoacrylate) with underlying tissue. The polymerization reaction is exothermic, and the rate of polymerization is inversely proportional to the amount of the monomer. The reaction rate is also inversely proportional to the length of the alkyl-side chain (Bhatia 2010). Longer carbon side-chain lengths yielded better elasticity, reduced adhesive strength, and lowered cytotoxicity (Mizrahi et al 2011). In general, the strength and other physical properties of the cyanoacrylate adhesives are directly related to the length and complexity of their alkyl-side chain. Short, straightchain derivatives (ethyl- or butyl-cyanoacrylate) form tight and stronger bonds compared with complex or long-chain derivatives (propoxy propyl cyanoacrylate and octylcyanoacrylate) (Singer et al 2008).

\section{Removal}

The adhesive film generally sloughs off within 5-10 days as the epidermis regenerates, so there is no need to remove the adhesive. Cyanoacrylate glues undergo hydrolytic degradation which takes place through non-enzymatic reactions; the main degradation products are formaldehyde and the corresponding alkyl-cyanoacetate (Bhatia 2010). The side chain is also responsible for the set time and degradation rate of the polymer (faster with short chains). Although formaldehyde is known to be a histotoxic irritant, any significant degradation occurs long after the adhesives have sloughed off the skin and these breakdown products do not contribute to any toxicity when cyanoacrylate adhesives are used topically (Singer et al 2008).

\section{Advantages}

The application of the adhesives is relatively rapid and painless. No local anesthetic is necessary and the amount of time saved is magnified in proportion to the length of the wounds. Thus the greatest time savings were for the longest wounds (Singer and Thode 2003). Tissue adhesives do not require special instrumentation or a removal procedure, thus there is no risk of needlestick injury to the surgeon or assistant. They decrease the amount of required wound care by serving as their own dressings (Bruns and Worthington 2000). Furthermore, wound closure with tissue glues, as opposed to traditional methods, requires less training (Trott 1997), as the cosmetic result of wound closure with tissue adhesive was judged independently of the surgeon's experience ( Quinn et al 1993).

The use of tissue adhesives is particularly helpful in wounds that are under splints or casts since they do not require removal and result in minimal if any irritation. The tissue adhesives are also particularly well suited for the closure of flaps and fragile skin since they do neither tear through tissues nor strangulate them, as do the encircling sutures. They may also be used in place of sutures or staples to secure partial thickness skin grafts (Singer and Thode 2004, Singer et al 2008). 
The cyanoacrylate tissue adhesives have also been shown to have a barrier function against microbial penetration and serve as an optimal wound dressing that creates a moist wound environment, thus enhancing wound healing (Singer and Thode 2004, Singer et al 2008). They may well be the most appropriate form of wound closure because they do not leave any foreign material that may serve as a carrier or reservoir of infection in the wound (Aksoy et al 2006).

\section{Limitations}

However, tissue adhesives should not be used alone for the closure of high-tension wounds (Singer et al 2008). Wound edges which are more than $5 \mathrm{~mm}$ apart due to the underlining skin tension are unlikely to stay in apposition with tissue adhesives alone and should be supported with subcutaneous sutures (MacPhail 2014). Wounds over joints should be immobilized if closed with tissue glues to prevent early peeling of the adhesive caused by excessive motion (Bruns et al 1998). Furthermore, adhesives should not be used for bite wounds, severely contaminated wounds, ulcers, puncture wounds, wounds which are near the eye or in areas with high moisture content (MacPhail 2014). Since repeated exposure to moisture may facilitate premature sloughing of the adhesives, tissue adhesives should be avoided over mucous membranes and over areas exposed to frequent moisture and/or friction such as the hands and feet (Singer and Thode 2004, Singer et al 2008).

Because they break down to formaldehyde, the cyanoacrylate tissue adhesives are contraindicated in patients with an allergy to the cyanoacrylates or formaldehyde (Singer et al 2008).

\section{Indications}

The tissue adhesives are indicated for any low-tension surgical incision or laceration whose edges are easily approximated with the operator's fingers or forceps, regardless of their length. Many long surgical incisions are deep and are closed in multiple layers, distributing the tension over a much larger area and resulting in less tension per unit of length, reducing the amount of tension on the most superficial layers, thus making the tissue adhesives an ideal alternative for closing the outermost layers of the skin. The butyl-cyanoacrylates have generally been limited to lacerations and incisions shorter than 4-8 cm in length (Singer and Thode 2004).

While the use of tissue adhesives might seem intuitive, there are most useful when they are used on wounds that close spontaneously, have clean or sharp edges, and are located on clean, non-mobile areas (MacPhail 2014). Improper wound selection and technique may result in suboptimal results (Singer and Thode 2004). The conditions for the application of tissue adhesives are intraoperative bleeding control to obtain optimal adhesion of the skin surface, subcutaneous sutures in deep tissue defects to minimize tension in both the epidermis and the film of the glue, and crack-free adaptation of wound margins to avoid a flow of glue in the wound region. Toxic reactions of liquid glues in patients are possible only in adapted wound edges with cracks (Nitsch et al 2005). 


\section{Clinical trials}

When clinically compared with sutures alone (Quinn et al 1993, 1998, Toriumi et al 1998, Mattick 2002, Holger et al 2004, Nagpal et al 2004) or with several traditional methods (sutures, staples, or adhesive tape) (Singer et al 2002, Chow et al 2010) for closure of surgical and/or traumatic wounds, tissue glues were credited for faster (Quinn et al 1997, Toriumi et al 1998, Singer et al 2002, Mattick 2002, Nagpal et al 2004, Chow et al 2010) and less painful closure (Quinn et al 1997, Mattick 2002), similar [Quinn et al 1998, Singer et al 2002, Holger et al 2004 ], improved [Toriumi et al 1998, Nagpal et al 2004] or worst (Song et al 2012) cosmetic outcome, and similar (Toriumi et al 1998, Singer et al 2002) or higher (Chow et al 2010) rates of dehiscence. Other favorable reports of tissue glues focused on applications including surgical incisions of the head and neck (Maw et al 1997, Bozkurt and Saydam 2008, Amin et al 2009), parotidectomy wounds (Greenhill \& O’Regan 2009) in external dacryocystorhinostomy (Hovaghimian et al 2015) and in surgical closing of postparotidectomy salivary fistulas (Pesic Z et al 2011), elective surgical incisions in a paediatric population (Amiel et al 1999, Arunachalam et al 2003, Brown et al 2009, Elemen et al 2011), blepharoplasty (Greene et al 1999), lacerations at various sites (Applebaum et al 1993, Bruns et al 1998, Singer et al 1998, Mattick 2002)] and in extremities that were immobilized to decrease high skin tension [Saxena \& Willital 1999], laparoscopic trocar site wounds (Rosin et al 2001), wound closure in cervical and lumbar spinal surgery (Wachter et al 2010), wound closure in brain surgery (Chibbaro \& Tacconi 2009), in cleft lip repair (Magee et al 2003, Knott et al 2007), in primary closure of sacrococcygeal pilonidal disease (Othman 2010).

\section{Surgical skin staples}

Use of mechanical stapling instruments is an effective substitute for manual suturing of a number of tissues (Boothe 2003). The first stapling instrument was invented in 1908 by Professor Humer Hultl, known as the father of surgical stapling. He developed an instrument to place straight double rows of staples (B-shaped and made of fine wire), in an alternating manner across the stomach (Doshi 1992, Robicsek et al 2001, United States Surgical Corporation History). In 1951 a stapling instrument for use in vascular surgery was developed at the Scientific Research Institute for Experimental Surgical Apparatus and Instruments in Moscow and was introduced into the United States, by Ravitch in the 1960s (Doshi 1992, Murphy et al 2004, Tobias 2007, United States Surgical Corporation History). In 1978, pre-assembled disposable stapling instruments were developed (Doshi 1992). Surgical staplers have a common principal design. They include a pusher system that advances a U-shaped wire through two or more tissues to be joined and into an anvil that bends the wire in some fashion to secure them (De la Cruz \& Cohn 2011).

Staples have been used in various surgical procedures, and they are having an increasing impact on noncardiac laparoscopic and minimally invasive lung, gastrointestinal and colorectal surgery (De la Cruz \& Cohn 2011, Konstantinov 2004).

Staples have been used in veterinary medicine for pulmonary, gastrointestinal, vascular, and skin procedures and may also be used for subcutaneous or fascial 
closures or vascular anastomosis (absorbable subcuticular staples) (O'Brien 1998, MacPhail 2002, Tobias 2007, Dresner and Hilger 2009, MacPhail 2014).

One of the first types of a surgical staple to become available was the skin staple, which has been used in wound closure for 40 years. The primary utility of staples is in the closure of wounds under high tension on the trunk, extremities, and scalp. Skin staples are made of inert 316L stainless steel. The width and height of the staples vary according to the manufacturer. Most regular staples are 4-6 mm wide and 3.5-4 mm high and wide staples for use in thicker skin are 6.5-7.5 mm wide and 4-5 mm high with a diameter of 0.51-0.58mm (Tobias 2007, Dresner and Hilger 2009, De la Cruz \& Cohn 2011, Satteson et al 2015), combining the advantage of high tensile strength with low tissue reaction (Thomas 2005). The non-crushing action of the stapler as it crimps the staple causes minimal tissue trauma (Trout 2003). The development of mechanically satisfactory skin staplers appears to be an important advance in rapid and effective means of primary wound closure of long skin incisions, skin grafts and scalp incisions (Gatt et al 1982, Stegmaier 1982, Tobias 2007).

\section{Application}

Before application, the skin staple has a straight cross member with 2 shorter, perpendicular, straight or curved legs. During application, the cross member is bent over an anvil, crimping it at 2 sites, bringing the legs together to form a rectangular shape that is narrower than the original staple. When the staple is placed it simulates a rectangular shape (an arcuate or triangular configuration is less common). A gap exists between the staple and the skin incision to allow postoperative tissue swelling (Trout 2003). Subcutaneous sutures should be inserted first in order to minimize tension at the wound margins for optimal cosmesis. Each stapler should engage the full thickness of the tissues being joined together. Before inserting the staples, it is important to line up the wound edges with the centerline indicator on the head of the stapler, thus making certain that the legs of the staple will enter the skin at the same distance from the wound edges on either side (Stegmaier 1982). The correct placement of staples is critical to avoid complications such as tissue strangulation and crosshatch marking. Staples should be inserted at $45^{\circ}$ or $60^{\circ}$ angles. As a wound swells, a staple placed at an acute angle rotates into a vertical position, leaving a space between the crossmember and the skin surface to accommodate swelling. If placed at a $90^{\circ}$ angle, the staple can't move and is likely to strangulate the tissue during swelling (Satteson et al 2015). In the case of long incisions requiring 10 or more staples, it is convenient to place a staple in the center and then closing first one and then the other part of the wound from the two ends inward (Stegmaier 1982). The ideal distance between staples, in a region subject to early active movement, is the result of a balance between achieving optimum wound oxygen tension levels and secure mechanical closure. Wound oxygenation characteristics are similar in subcuticular vicryl and stapled skin closure with an interstaple distance of $6 \mathrm{~mm}$. Greater spacing between staples is beneficial in terms of oxygenation but the surgeon must be certain that the wound closure is stable (Graham et al, 2000). The stapler is disposable, but it can be sterilized and reused if only a few staples are used for a given procedure. An instrument with 
thirty-five or fifty-five staples can be cleaned ultrasonically and resterilized in the autoclave until most of the staples have been used (Stegmaier 1982).

\section{Removal}

Skin staples should be removed at the same time that sutures would be removed, based on wound location and tension. For scalp wounds in humans, staples should be removed on day 7 after insertion and for trunk and extremity wounds, between days 67 and 12-14 (Stegmaier 1982, Dunn and Phillips 2004). Staples removal is significantly faster than that of sutures (Murphy et al 2004). A specifically designed staple extractor is optimal to compress the cross-member of the staple straightening and painlessly opening the legs to permit easy extraction in one motion. If necessary, a mosquito haemostat can be used to open the staple before removal (Gatt et al 1982, Stegmaier 1982, Teoh 1987, Trout 2003).

\section{Advantages}

Staples provide a rapid, accurate, and everted skin closure (Trout 2003). Potential benefits of stapling include reduction in surgical time, tissue trauma, and intraoperative contamination, and preservation of vascular supply (Tobias 2007). Staples can be placed faster than sutures and have a lower predisposition to infection as they do not penetrate entirely through the wound and do not produce a complete track from one wound edge to the other (Thomas 2005). An additional advantage of staples is their low level of tissue reactivity. There is uniform agreement that wounds closed by metal staples exhibit a superior resistance to infection than wounds subjected to the least reactive suture (Edlich et al 2010). Experience with the technique is likely to increase even more the speed of staple closures as compared to suture closures (Shuster 1989). The use of staplers for skin closure allows saving in time, it can speed up closure by $80 \%$ which is an important factor especially for the closure of large or multiple incisions, and neither compromises the final cosmetic result nor increases the incidence of complications (Medina dos Santos et al 1995). Time-saving with staples may not be of importance in an elective setting but may be more relevant in an emergency situation in the setting of a busy labor and delivery unit (Cromi et al 2010). Moreover, a short scalp laceration can be quickly stapled without anesthesia: one or two staples take less time to apply and seem to cause less discomfort than the injection of anesthetic (Shuster 1989). The superficial part of the staple rarely lies in tight approximation to the healing skin edge, unlike a simple interrupted suture. The 'open rectangle' formation of the staple allows for tissue expansion and thus contributes to the absence of 'cross-hatched' scarring (Graham et al 2000). No increased predisposition to infection is apparent with reuse of the stapling device. Local suture removal is necessary for moderately infected wounds to allow drainage but staples allow satisfactory drainage without removal (Gatt et al 1982). Moreover, other potential benefits of using staples is a reduction in the risk of needlestick injury to the surgeon and assistant (Cromi et al 2010), and the fact that no postoperative dressing is required (Stegmaier 1982). Patients may bath or shower the next day but should avoid prolonged exposure to moisture (Dunn and Phillips 2004). 


\section{Limitations}

Staples are not ideal for the closure of wounds under moderate tension because they present a higher risk of wound dehiscence. Furthermore, they are not placed over bone or viscera if the distance between the skin and the underlying tissues is less than 4-6.5 $\mathrm{mm}$ (Trout 2003). They should not be used in delicate tissues or wounds in finely contoured areas (Satteson et al 2015). The staples are uncomfortable at groin wounds or on gross, uneven surfaces (Stegmaier 1982). Finally, skin staples do not provide the same meticulous cooptation of lacerations with irregular skin edges as sutures do. Wound edges must be accurately aligned before wound closure to permit simultaneous implantation of the staple points. Because accurate pre-positioning of wound edges is very difficult in most lacerations, staple implantation will often result in malapposition of wound edges, resulting in the development of scar deformity (Edlich et al 2010).

As with conventional suturing, surgical stapling will not be successful if staples are improperly applied (Stegmaier 1982). Poor technique in staple placement, such as the application of a staple at an angle, may result in overlapping or inverted wound edges that lead to oozing from wound edges, delays healing and possible promotes infection. So, an assistant who everts wound edges with toothed forceps in advance of the skin stapling may be beneficial (Murphy et al 2004). Furthermore, if a staple sits deep in the tissue, so that the cross-member is in contact with the skin, the staple can become embedded in the incision during normal postoperative swelling, and cross marks may result (Trout 2003). Sometimes a partial removal of staples after several days can be helpful, while complete removal is considered safe after at least two weeks (Mondini et al 2012). In some staples, the pointed ends almost touch, whereas certain types retain a distinct space that may allow greater tissue swelling without vascular obstruction. Unfortunately, this space may permit excessive motion or rotation of the staple, particularly in the thin skin of cats, in which it can rotate through $90^{\circ}$, during normal body movement (Trout 2003).

\section{Indications}

Staples are ideal for closing scalp incisions. They are also indicated for linear nonfacial lacerations caused by shear forces (sharp objects) and surgical incisions of the torso and extremities, especially if they are relatively long. The staples provide eversion of wound edges so that subcutaneous suturing is less often necessary but in any case in which optimal cosmesis must be sought, subcutaneous sutures should be inserted first in order to minimize tension at the wound margins (Stegmaier 1982, Dunn and Phillips 2004). In humans, skin staples are reserved for lacerations in anatomic sites in which the healing scar is not readily apparent (e.g., hair-covered scalp) (Edlich et al 2010). The peripheries of split-thickness skin grafts can be secured with staples (Stegmaier 1982). In patients at substantial risk for intraoperative contamination or morbidity as a result of prolonged anesthesia, use of stapling devices should be considered (Tobias 2007). 


\section{Clinical trials}

When clinically compared with sutures alone (Gatt et al 1982, Shuster 1989, Ranaboldo et al 1992, Medina dos Santos et al 1995, Kanegaye et al 1997, Moore et al 1999, Rousseau et al 2009, Clay et al 2011) or with several traditional techniques (sutures, glue) (Khan et al 2006), staples were credited for faster closure, and faster and less painful removal (Gatt et al 1982, Murphy et al 2004). When clinically compared with sutures (Stockley 1987, Shuster et al 1989, Medina dos Santos et al 1995, Murphy et al 2004, Obermair et al 2007, Gaertner et al 2008, Rousseau et al 2009, de Graaf et al 2012) or with glues (Ridgway et al 2007, Amin et al 2009, Cromi et al 2010, Ong et al 2010, Pronio et al 2010) or with several traditional techniques (sutures, glues or adhesive tape) (Singer et al 2002, Blondeel et al 2004, Khan et al 2006, Chibbaro and Tacconi 2009, Eggers et al 2011, Mondini et al 2012) ) they were credited with having similar wound outcome.

In orthopaedic and cardiovascular surgery, staple closure was associated with a significantly higher risk of superficial wound infection compared to sutures (Stockley 1987, Johnson et al 1997, Sanni and Dunning 2007, Basha et al 2010, Smith 2010), or fewer risk (Newman et al 2011), or no association between closure type and wound complications was found (Murphy et al 2004, Khan et al 2006, Eggers et al 2011, Mondini et al 2012). In obstetrics/gynecology, abdominal, head/neck, and vascular operations, staples were found to be superior to sutures as far as the development of wound infections are concerned (Iavazzo et al 2011) or no difference was observed (Obernair et al 2007, Moore et al 1999).

\section{Cutaneous ultrasonography}

Since the discovery of X-rays, imaging technology has an important role in medicine. Technological advancements have led to the development of various imaging modalities, most of which have been used to image organs deep within the body. Significant research to further develop techniques for specifically examining the skin has been undertaken only in the last decades. Advances in both the technology of imaging instruments and computer systems have greatly assisted this process and brought it closer to the clinical reality. Specialized photography, surface microscopy, ultrasound, laser Doppler perfusion imaging and magnetic resonance imaging are some of the techniques that are currently being used to specifically examine the skin (Manning et al. 1991, Aspres et al 2003, Tsioli et al 2015). Although many of these techniques are still under research, they are promising useful clinical tools in dermatology (Aspres et al 2003).

Traditionally, non-invasive estimation of wound healing has relied on photography. This provides a visual impression of the wound at the surface level only. It also allows an objective overview of the wound site, which can be displayed as a series of images to show the surface's changes that occur during healing. More detailed information on the state of the wound has relied on invasive skin biopsy, providing tissue samples that yield objective quantitative data relevant to the healing process. However, the sampling procedure inevitably produces further damage, and, as a result, has an impact on the healing process (Dyson et al 2003). 


\section{Principles of ultrasonography and instrumentation}

Ultrasonography has an established diagnostic role in many fields of medicine (Aspres et al 2003). It detects and displays interfaces between different organs or different tissue types and some internal tissue structures may also be detected. When the signal meets a boundary between two different media, a percentage of the sound is reflected, refracted or transmitted through the tissue (Rippon et al 1998). Transducers, which are thin disk-shaped crystals made out of piezoelectric materials, generate acoustic energy when a voltage is applied to them. Acoustic vibrations (frequencies) are generated when those piezoelectric materials expand and contract. Early transducers were made from quartz; newer materials include lithium sulfate, ceramics, and plastic polymers. These newer substances have allowed the development of transducers that produce higher frequencies, which are of special interest for dermatologists because the wavelengths of higher frequencies are smaller and, therefore, allow better resolution of small objects located near the skin surface. Currently, B-mode scans are the mainstay of all ultrasonographic procedures in dermatology, using intermediate- or highfrequency ultrasound systems (Schmid Wendtner and Burgdorf 2005). The depth to which sound penetrates into soft tissues is directly related to the frequency employed. Higher frequency sound waves are attenuated more than lower frequency waves. This means that any attempt to improve spatial resolution by increasing the frequency invariably decreases the depth of penetration (Nyland et al 2002). Terms that are used to describe the appearance of ultrasound images should relate to a tissue's echo intensity (echogenic, hyperechoic, hypoechoic and anechoic), attenuation (high and low), and image texture (homogeneous and heterogeneous) and relative to surrounding tissue or other structures (Nyland et al 2002).

Accurate interpretation depends directly on the differentiation of normal and abnormal anatomy. Normal parenchymal organs and tissues are visualized at various shades of gray, which are fairly constant. Diseases that diffusely involve organs or tissues may alter the usual echogenicity relationship (Nyland et al 2002). Clinical diagnostic ultrasound scanners use frequencies in the range of $1-20 \mathrm{MHz}$ (LiangBlomley 2003). Improvements in instrumentation have resulted in the development of scanners that can operate at much higher frequencies than when ultrasound biomicroscopy began, typically were 20MHz and higher (Dyson et al 2003). Ultrasound biomicroscopy (UBM) typically operates at frequencies of 40-200 $\mathrm{MHz}$, while scanning acoustic microscopy (SAM) uses frequencies higher than $200 \mathrm{MHz}$ (Liang and Blomley 2003). The ultrasonic visualization of living tissue at microscopic resolution is referred to in the literature as "ultrasound backscatter microscopy" or "ultrasound biomicroscopy” or "high-frequency ultrasound” or "very high-frequency ultrasound".

By using a $20 \mathrm{MHz}$ transducer, it is possible to visualize structures up to $6-8 \mathrm{~mm}$ in depth (Schmid Wendtner \& Burgdorf 2005, Bleve et al 2012). This means that the zones of diagnostic interest are covered, i.e. epidermis, corium, and a portion of subcutaneous fatty tissue. Particularly, if the subcutis is not very well developed, 
evaluation of the muscle fasciae is also possible (Bleve et al 2012). The use of the higher frequency range from 40 to $100 \mathrm{MHz}$ transducers, allows a more detailed examination of the epidermis and upper dermis. The maximum achievable frequency of commercially available equipment is $100 \mathrm{MHz}$; however, the penetration depth, in this case, is limited to $1.5 \mathrm{~mm}$ (Turnbull et al 1995, Raišutis et al 2010).

\section{Ultrasound appearance of normal skin anatomy and main uses of cutaneous ultrasonography}

Ultrasonographically, skin (both human and canine) is characterized by 3 distinct and recognizable layers. Most superficially, there is a well-defined and regular echoic line (epidermal entry echo). Because of its' typical, very low thickness, the epidermis appears ill-defined on ultrasound scans. The epidermis is a highly reflective structure and its echogenicity depends on the thickness of the stratum corneum and on the amount of air trapped between the desquamating corneocytes (Szymanska et al 2000). At $40 \mathrm{MHz}$ the unresolved stratum corneum/epidermis is shown as a hyperintense band overlying dermis (Foster et al 2000). At $50 \mathrm{MHz}$, the epidermis is clearly visible as a relatively thick, mildly non-uniform, hyperechoic linear layer. The thickness of this layer varies and probably depends on the angle of the ultrasound beam, the pressure applied and the examination site. The margin between epidermis and dermis is also visible (Mantis et al 2014), however, the lower margin at the epidermal-dermal interface is not distinct enough to allow accurate measurement of the epidermal thickness (Mantis et al 2014). A hyperechogenic layer corresponds to the dermis, where the superficial and deep layers can be differentiated with transducers of $13 \mathrm{MHz}$ or more: the first layer, slightly hypoechogenic and heterogeneous, corresponds in the histology sections to areas of the papillary dermis, and the second one, more hyperechogenic and homogeneous, corresponds to reticular dermis (Rippon et al 1998, Bouer et al 2008). At $50 \mathrm{MHz}$, the dermis in dogs has a granular echotexture that appears to become more linear in the deeper parts, though this distinction between the superficial and deep parts of the dermis is not always apparent. The superficial dermis has a granular appearance more loosely arranged, with thin and irregularly distributed small echoes, that probably arises from the collagen fibers. The deep dermis contains thicker linear echoes oriented more parallel to the skin surface; however, this distinction is not always clearly visible (Mantis et al 2014). In healthy skin small, hypoechoic areas which correspond to hair follicles, vessels, and sebaceous glands, can be imaged (Szymanska et al 2000, Bouer et al 2008). At 50MHz, in dogs, the hair follicles, especially their isthmus and inferior segment, are regularly identified. Their appearance varies depending on the orientation of their axis in relation to the orientation of the ultrasound beam. When the hair follicles are viewed along their long axis they appear as oblique, roughly tubular hypoechogenicities with faint echogenic linear areas at the margins, while when viewed in an oblique fashion they appear as mildly irregular in outline oblong hypoechogenicities. The fat around the root of the anagen hair follicles appears as a moderately indistinct in margination "cloudy" hypoechogenicity (Mantis et al 2014). 
Dermal echogenicity depends on the number of collagen fibers that increase dermis echogenicity and lessen echogenic intercellular matrix. Water influx causes a decrease in the echogenicity, probably due to distension of the fiber network (Szymanska et al 2000, Bouer et al 2008). In humans, the dermis echogenicity varies with age; it appears hypoechogenic in neonates, with a mild increase in echogenicity in older infants, and shows a pronounced increase in adults. In the elderly, a more echogenic layer is observed at the level of superficial dermis, mainly because of exposure to the sunlight (Bouer et al 2008). A subepidermal nonechogenic band appears with age, which is a sign of skin-aging (chronological and photoinduced) (Vaillant 2004). This likely corresponds to elastosis, changes in collagen structure, and water accumulation (Waller and Maibac 2005). Skin roughness parameters increase with age and had a strong correlation with dermal density and skin thickness (Lee et al, 2008). In general, echogenicity is decreased in dermal oedema, but there are marked differences in the distribution of the low echogenic region in various diseases (Szymanska et al 2000, Diana et al 2008).

Skin thickness in humans, varies according to body area (extremity skin is thinner than truncal skin), patient's age (skin thickness increases from birth to the age of 15years and reduces after 60 years of age and at 80years in less than infants) and sex (female skin is thinner than male skin) (Olsen et al 1995, Diridolloy et al 2000, Szymanska et al 2000, Vaillant 2004). The apparent thickness of skin varies slightly with the amount of pressure on the transducer, with the dermis becoming thinner and the echogenicity of the dermis increasing slightly as the pressure increases (Fornage et al, 1993). Canine skin thickness has been reported to decrease dorsally to ventrally in the trunk and proximally to distally in the limbs, the thickest skin located on the head, dorsum of the neck, back and sacrum (Mantis et al 2014). Diana et al (2008) ultrasonographically $(13 \mathrm{MHz})$ confirmed that skin thickness in dogs varies at different cutaneous sites within an individual dog and that skin hydration is positively correlated with skin thickness in this animal species. In particular, among the cutaneous sites she examined, the epidermis-dermis zone of the sacral region had the greatest thickness and skin was less thick in the frontal, flank, and metatarsal regions (in decreasing order). Moreover, dermal echogenicity and the double-layered appearance of the dermis (the deeper layer of which is less echogenic than the more superficial layer) were correlated with cutaneous fluid content. (Mantis et al (2014) found no significant differences in ultrasonographically estimated skin thickness between male and female dogs, as well as between dogs of different age. Zana et al (2012) observed a weak correlation between ultrasonographically measured skin thickness and sex, and skin thickness in the dorsal neck and frontal regions and age in Beagles. However, no correlation was found between ultrasonographically measured skin thickness and sex in Shar-Peis.

At greater depth, there is the thicker layer of subcutaneous tissue (Zana et al 2012), characterized by an inhomogeneous hypoechoic or non-echogenic pattern (compared with the overlying layers), containing thin linear hyperechoic areas or bands representing connective septa (adipose nature) (Fornage et al 1993, Szymanska et al 2000, Bouer et al 2008, Diana et al 2008, Mantis et al 2014). 
One of the main uses of cutaneous ultrasonography is in the measurement of skin thickness, which has been shown to be reliable and accurate in dogs (Mantis et al 2005, 2007, Diana et al 2008, Mantis et al 2014) and humans (Fornage et al. 1993, Olsen et al 1995, El Gammal et al 1999, Diridolloy et al 2000). Ultrasonography has been also used to assess skin-thickness changes in a variety of skin diseases, as scleroderma (Szymanska et al 2000, Bouer et al 2008), localized morphea (Szymanska et al 2000) and psoriatic lesions (El Gammal et al 1999, Szymanska et al 2000), photodamaged and chronologically aged skin (Crisan et al 2012, Gniadecka 2001), to calculate nail plate and nail matrix parameters in patients with skin disease (Wollina et al 2001), to investigate the effect of hormone replacement therapy on skin thickness (Chen et al 2001), to asses cutaneous changes induced by topical flavonoid therapy (Crisan et al 2012), to serve as a potential assessment technique for irradiated skins (Huang et al 2007), to examine hypertrophic scars, providing an innocuous way to objectively visualize the dynamics of the lesions while undergoing different therapeutic approaches (Bessonart et al 2005), to support management of skin and nail lesions under common rheumatologic conditions (Wortsman et al 2011), to evaluate the use of an aliamide-containing gel in the treatment of skin wounds (Mantis et al 2007), to assess the possibility of osteoporosis or osteopenia improving the predictability of bone density levels in women (Cagle et al 2007), and to evaluate skin hydration status and pathologic modifications of skin hydration in dogs (Diana et al 2008).

However, high-frequency ultrasound has not helped in the differentiation of benign from malignant skin lesions (Fornage et al. 1993) but contributed to the assessment of skin tumours by providing information on tumour depth and margins. These features may be useful in the preoperative evaluation of skin tumours and in monitoring the response to therapy of certain inflammatory conditions (Foster et al 2000).

High-frequency ultrasound has been employed for evaluating wounds, including burn scars, surgery wounds, and pressure ulcers (Moghimi et al 2011). In particular, ultrasound has allowed visualisation and quantification of wound healing components such as collagen accumulation, reepithelialisation and wound area, in vivo and noninvasively, which is an attractive alternative to biopsy in the clinical environment. Measurements using this technique are likely to provide a more accurate indication of tissue thickness than histological measurements that were subject to changes postfixation and post-processing (Rippon et al 1998). High-frequency ultrasound scanning permits the quantitative assessment of structural changes deep within the wound and those temporal changes in the width of the wound base can be used as an indication of the progress of repair (Dyson et al 2003).

\section{B-scan ultrasound wound area measurement of skin in the dog}

With a normal use, canning does no damage the wound and allows serial scans to be taken so that temporal changes within the wound can be monitored (Dyson et al 2003). High Resolution Ultrasound (HRU) enables differentiation between the wound area and the surrounding healthy tissues during wound healing. Even when the formation of eschar obscures the clinical evaluation of small healing wounds, they could be readily visualized by HRU (Mantis et al 2005). The main clinical role of ultrasonography has 
been in the objective monitoring of disease progression, with serial measurements allowing the effectiveness of treatment in patients to be assessed more accurately (Aspres et al 2003). 
Institutional Repository - Library \& Information Centre - University of Thessaly 26/04/2023 13:56:30 EEST - 54.147.182.235 


\section{PART TWO}

\section{MATERIALS AND METHODS}

The study was performed in the research facility area of the Department of Surgery, Faculty of Veterinary Medicine, University of Thessaly. The research protocol of the study was approved by the Greek National Animal Ethics Committee (license number: 1174/13.4.2009), as being in accordance with the European Union legislation concerning humane animal treatment and welfare of laboratory animals. Throughout the study, all legal and ethical requirements with regards to humane treatment of animals have been met.

\section{Dogs}

Ten healthy purpose-bred laboratory Beagles (5 males and 5 females), aged 1-5 years old, from the breeding colony of the Department of Surgery, Faculty of Veterinary Medicine, University of Thessaly, were included in the study. All dogs were housed individually during the first month of the experiment, with access to outdoor pens permitting eye-contact between animals. Following this period of privacy, they were housed as before, i.e. in groups of 3-4 dogs.

\section{Inclusion criteria}

The dogs were healthy clinically and dermatologically, without active skin lesions, without traumas or scars in the areas to be examined (old post-traumatic scars in other areas of the skin were permitted) and without evidence of infection or systemic disease. They had received no medical treatment during the study and the previous 30 days, with the exception of antibiotics and opioids, given during the first postoperative days, as well as the annual vaccinations and the routine antiparasitic medication.

\section{Initial and continuous evaluation of health status}

Before and during the experiments, each dog's health status was evaluated by physical and laboratory examinations; physical examinations were performed every fifteen days and laboratory examinations every six months. Complete blood count, biochemistry examination of serum for measurement of total proteins, albumins, globulins, urea nitrogen, creatinine, glucose, alkaline phosphatase, alaninoaminotransferase, serological examination for canine leishmaniosis, monocytic ehrlichiosis and Dirofilaria immitis and urinalysis. Throughout the study, the animals were subjected periodically to all required vaccinations and preventive antiparasitic treatment for endoparasites and ectoparasites. They were housed in enclosures of appropriate area size and had free outdoor access. They were fed a standard commercial dry maintenance diet and water was offered ad libitum.

\section{Experimental protocol}

Two incisions, $12 \mathrm{~cm}$ long, were made through the skin and subcutaneous tissue at the lateral surface of each thigh, which were parallel to the long axis of the femur, and 7 
cm apart from each other. Afterwards, in all incisions, the subcutaneous tissue was closed primarily with continuous subdermal suture pattern by using 3-0 polyglactin 910 (Vicryl, Ethicon Inc, Somerville, NJ). Secondary, the skin of each incision was closed with a different technique, which was selected after opening a sealed envelope. Skin staples (Proximate, Ethicon Inc, Somerville, NJ) [staples], n-butyl cyanoacrylate tissue glue (Vetbond, 3M) [glue], continuous intradermal suture pattern (with burying of the knot) by using 4-0 poliglecaprone 25 (Monocryl, Ethicon, Inc, Somerville, NJ) [intradermal B] and continuous intradermal suture pattern with clips (without burying the knot) by using 4-0 polypropylene (Securex, B Braun Aesculap AG \& Co KG, Tuttlingen, Germany) [intradermal C], were used. The time required to perform each technique was recorded.

\section{Anaesthesia and surgical procedure}

Dogs were premedicated by intramuscular injection of dexmedetomidine (300 $\mu \mathrm{g} / \mathrm{m}^{2}$, Dexdomitor, Pfizer Hellas, Greece) and morphine $(0.5 \mathrm{mg} / \mathrm{kg}$, Morphina cloridrato, Molteni Pharmaceutici, Florence, Italy). After 30 minutes, anaesthesia was induced by intravenous administration of thiopental sodium 2.5\% (5-7 mg/kg, Pentothal, Abbott Hellas, Greece), and, after intubation of the trachea, it was maintained with isoflurane (1.5-2\%, Aerrane, Baxter, UK) in oxygen (1 L/min), using a semi-closed anesthesia circuit. During anesthesia Lactated Ringer's solution $(10 \mathrm{ml} / \mathrm{kg} /$ hour, Lactated Ringer's, VIOSER, Greece) was administered by continuous intravenous infusion.

After hair clipping of the lateral side of the thighs, surgical field scrubbing with antiseptic soap containing chlorhexidine gluconate (Hibitane scrub 4\%, Cana SA, Greece) and spraying with antiseptic solution of $0.5 \%$ chlorhexidine gluconate and isopropyl alcohol 60\% (Hibitane alcoholic solution, Cana SA, Greece), surgical drapes were placed. A sterile ruler and a sterile marker were used to mark the incision lines. Then two $12 \mathrm{~cm}$-long skin and subcutaneous tissue incisions were performed with a No 10 lancet at the lateral aspect of each thigh, the distance between them being $7 \mathrm{~cm}$. After vascular clamping with haemostatic forceps for bleeding control, the chosen skin closure technique was performed. The procedure was performed initially at the right thigh and subsequently at the left thigh.

In the skin stapling closure method, the staples were placed along the surgical incision in such a way that the distance between them was $8 \mathrm{~mm}$ and the distance from the wound edges was 6-7 mm.

In the tissue adhesive application, the n-butyl glue was applied to the apposed wound edges as a thin, single and continuous layer. Care was taken to avoid adhesive pooling or thick application of glue.

In the continuous intradermal suture pattern (with burying of the knot), the initial knot of suture material was buried in the subcutaneous tissue at a distance of $4 \mathrm{~mm}$ from the commissure of the wound, and then the suture material was directed at the start of the incision in the middle of the dermis. The course of suture was followed in the middle of the dermis, and special care was taken that the suture material did not pass through the basal lamina of the epidermis. Approximately $2.5 \mathrm{~cm}$ before the end 
of the wound, the suture was anchored with a buried knot, so that a biopsy could be performed at the last $2 \mathrm{~cm}$ of the suture line, without the collapse of the entire suturing. At the last passage of the suture from the dermis, the needle was directed backward and the suture was fixed with an Aberdeen knot (6 loops) in the subcutaneous tissue. After knot formation, the tip of the suture material was directed to the subcutaneous tissue at the lateral side of the incision and finally outside the skin, so that the knot was moved away from the skin healing layer and the suture material was cut at the level of the skin surface.

In the continuous intradermal suture pattern (without burying of the knot), the suture material was anchored to one end of the skin incision with a fixation clip just before the commissure of the surgical skin incision and the needle was passed from this point through the wound commissure. The suturing was continued by passing the suture material horizontally in the middle of the dermis. Approximately $2.5 \mathrm{~cm}$ before the end of the wound, the suture was anchored with an extra clip, so that a biopsy could be performed at the last $2 \mathrm{~cm}$ of the suture line, without the collapse of the entire suturing. On the final bite, suture material passed through the full thickness of the skin and was secured through a third fixation clip.

\section{Postoperative treatment}

Immediately after surgery and until the $10^{\text {th }}$ postoperative day (po.d.), Elisabeth collars were placed on the dogs and bandages were applied on the thighs in order to protect the wound site. On the $10^{\text {th }}$ po.d., the staples and polypropylene suture material with clips were removed. Dogs received morphine (Morphina cloridrato, Molteni Pharmaceutici, Florence, Italy) at a dose of $0.5 \mathrm{mg} / \mathrm{kg}$, im, every four hours for 3 postoperative days and every six hours for the following 4 postoperative days, while until the $10^{\text {th }}$ po.d., they also received amoxicillin and clavulanic acid (Synulox) at the dose rate of $12.5 \mathrm{mg} / \mathrm{kg}$, sc, twice daily.

\section{Evaluation of wound healing of skin incisions \\ Cosmetic evaluation}

In order to access the cosmetic appearance of wound healing, photographs (Digital High Resolution Camera (DSC-T3, Sony) of the wound area were taken before the surgical skin incision, immediately after skin closure (Day 0), every day until the $10^{\text {th }}$ po.d., on the $12^{\text {th }}, 14^{\text {th }}, 16^{\text {th }}, 18^{\text {th }}, 21^{\text {st }}, 24^{\text {th }}$ and $28^{\text {th }}$ po.d., once a week until the end of the $3^{\text {rd }}$ postoperative month, and once a month until the end of the experiment, i.e. one year after the incision. The closed skin was photographed in its entirety and at the central part of it (4-5 cm long). A $3.2 \mathrm{~cm}$ long label, with information about the animal identity, the postoperative date and the technique used, was placed next to the incision line for future identification and measuring.

Cosmetic appearance was blindly evaluated by two experienced surgeons (P. Gouletsou, A. Galatos) on a 1-10 visual analogue scale (1: excellent cosmetic result, 10: bad cosmetic result), by assessing the photographs (with the labels of photographs covered) of the $7^{\text {th }}, 14^{\text {th }}, 28^{\text {th }}, 63^{\text {rd }}, 91^{\text {st }}, 120^{\text {th }}, 150^{\text {th }}, 180^{\text {th }}, 210^{\text {th }}, 240^{\text {th }}, 270^{\text {th }}, 300^{\text {th }}$, $330^{\text {th }}$ and $365^{\text {th }}$ postoperative days. The scores from the two assessors were averaged 
to generate the total cosmetic appearance score for each wound. The higher the total score, the poorer the wound cosmetic appearance.

\section{Clinical evaluation}

Clinical evaluation was performed immediately after surgical skin closure (Day 0), every day until the $10^{\text {th }}$ po.d., on the $12^{\text {th }}, 14^{\text {th }}, 16^{\text {th }}, 18^{\text {th }}, 21^{\text {st }}, 24^{\text {th }}$ and $28^{\text {th }}$ po.d., once a week until the end of the $3^{\text {rd }}$ postoperative month, and once a month until the end of the experiment. The following parameters were evaluated:

1. Oedema (skin thickening at the central part of the incision, measurement by skin caliper, in mm)

2. Erythema, (width of skin redness at the central part of the incision, measurement by electronic calipers, in $\mathrm{mm}$ )

3. Scar width in the central part of the incision (measurement by electronic calipers, in $\mathrm{mm}$ )

4. Abscessation or inflammation (0: absence, 1: mild inflammation, 2: 1-2 microabscesses, 3: strong inflammation, or more than 3 microabscesses)

5. Exudate (0: absence, 1 : serosanguineous, 2: seropurulent, 3: purulent)

6. Comedones (0: absence, 1: 1-3, 2: 4-6, 3: more than 7)

7. Hyperchromia of the wound area (hyperpigmentation), compared with the adjacent skin color (0: absence, 1: mild, 2: moderate, 3: intense)

8. Staples loss (number of staples detached)

9. Wound dehiscence (length in $\mathrm{cm}$ )

10. Cross scaring (0: absence of skin marks, 1: 1-2 marks, 2: 3-6 marks, 3: more than 7 marks).

If skin thickening, erythema and scar width were uneven along the incision, more than one measurement was taken and the average value was used.

\section{Ultrasonographic evaluation of the healing process}

B-mode real-time ultrasonographic examination of the skin was performed by the use of a real-time ultrasound machine. The ultrasound unit (Longport Digital Scanner [LDS1], Longport International Ltd., Silchester, United Kingdom) was fitted with a $50 \mathrm{MHz}$ polyvinylidene difluoride transducer incorporated into a probe filled with distilled water and scanned using a digital stepping motor. The ultrasound beam was propagated through an aperture covered with a disposable rubber membrane; a new membrane was used for each wound. The transducer was applied to the wound area using light pressure and coupling gel as a transmission medium. Scans were taken perpendicular to the long axis of the incision and to the adjacent intact skin. Four transverse images (a, b, c, d) were taken from each wound, in such a way that the distance between the scans was $2-3 \mathrm{~cm}$. Wounds were examined daily until the 10 th po.d., on the $12^{\text {th }}, 14^{\text {th }}, 16^{\text {th }}, 18^{\text {th }}, 21^{\text {st }}, 24^{\text {th }}$ and $28^{\text {th }}$ po.d., once a week until the end of the $3^{\text {rd }}$ postoperative month, and once a month until the end of the experiment. Furthermore, a scan was performed in the area where skin punch biopsy would be performed, just a few minutes before the biopsy. The digitized scans were stored on the associated hard drive and were visualized using a color palette (rainbow). Images 
were compressed laterally to facilitate viewing of the wound area. The wound area calculations were performed by using the computer software.

\section{Histological evaluation of the healing process}

Eight-millimetre punch biopsies (Scardino et al. 1998) were taken from selected sites of the incisions on the $7^{\text {th }}, 14^{\text {th }}, 28^{\text {th }}, 180^{\text {th }}$ and $365^{\text {th }}$ po.d. These time points were chosen in order to assess early and late stages of wound healing and scar maturation over time (Court and Bellenger 1989; Hunt et al. 2000; Molea et al. 2000; Ribeiro et al. 2005).

The biopsy specimens were fixed in buffered $4 \%$ formalin, cut in a direction perpendicular to the longitudinal axis of the incision, embedded in paraffin blocks, cut into 5 microns sections and stained with hematoxylin and eosin. Subsequently, these stained tissue samples were examined microscopically (simple observation) and skin healing parameters were evaluated. The evaluator knew only the biopsy timing but not the method of skin closure. These parameters were scored based on grading systems that have been used in relative studies (Kirpensteijn et al 1997, Gillette et al 2001, Winkler et al 2002, Gouletsou 2014). The histological variables were scored (0 to 3) at $40 \mathrm{x}$ or $400 \mathrm{x}$ magnification as follows:

1. Necrosis (0: none identified, 1: few scattered areas, 2: multiple focal dense areas, and 3: necrosis present throughout the slide) (Winkler et al. 2002)

2. Oedema (0: absence, 1: mild (slight) separation of cells and collagen fibers by nonstained or poorly stained acellular material [mild oedema], 2: by 30-50 microns separation [moderate oedema], 3: by more than 50 microns separation [marked oedema]) (Gillette et al 2001)

3. Inflammatory reaction, by counting the total number of inflammatory cells (neutrophils, eosinophils, macrophages, lymphocytes, plasma cells and mast cells) in 10 high magnification visual fields (400x) (0: less than 3 cells per field, 1: 3-10 cells per field 2: 11-30 cells per field, 3 : more than 31 cells per field) (Winkler et al 2002)

4. The thickness of the epidermis at the area of wound healing (number of times the thickness of adjacent healthy epidermis) (Kirpensteijn et al 1997, modified)

5. Epithelial gap (mm)

6. Scar width, as a region with a different appearance from healthy skin (in $\mathrm{mm}$ )

7. Suture material presence (0: no suture material identified, 1 : presence of a small part of the initial diameter of the suture material, 2: presence of most of the initial diameter of the suture material, 3: presence of the entire suture material)

8. Tissue reaction around the suture material (1: up to2 cell layers, 2: 3-5 cell layers, 3: 6-10 cell layers, 4: more than 10 cell layers) (Kirpensteijn et al 1997)

9. Fibroblasts' presence (0: less than 3 fibroblasts per 400x field, 1: 3-10 fibroblasts per 400x field, 2: 11-30 fibroblasts per 400x field, 3: more than 31 fibroblasts per 400x field) (Gillette et al 2001, Winkler et al 2002)

10. Collagen deposition (0: absence of collagen, 1: few scattered bundles of collagen separating fibroblasts, 2: dense collagen between fibroblasts, 3: severe fibroblast separation by collagen) (Gillette et al 2001, Winkler et al 2002) 
11. Angiogenesis (0: less than 3 capillaries per 400x field, 1: 3-10 capillaries per 400x field, 2: 11-30 capillaries per 400x field, 3: more than 31 capillaries per 400x field) (Winkler et al 2002).

\section{Statistical Analysis}

An initial exploratory analysis was conducted to ensure the fitting of the quantitative data to the normal distribution, with the use of the Shapiro Wilk test (Shapiro and Wilk 1965) (the size of each group was less or equal to 50). In order to compare the differences between the techniques over time, we formulated the Friedman two-way analysis by ranks dividing the experimental period into 5 time periods (Sheskin 2005). The periods were chosen to reflect the different phases of wound healing:

- Time period A $\left(1^{\text {st }}-8^{\text {th }}\right.$, when inflammation, debridement, and proliferation take place

- $\quad$ Time period B $\left(9^{\text {nd }}-21^{\text {th }}\right.$ po.d. $)$, when proliferation takes place

- $\quad$ Time period C $\left(22^{\text {nd }}-63^{\text {th }}\right.$ po.d. $)$, when the early stage of maturation takes place

- Time period D $\left(64^{\text {nd }}-180^{\text {th }}\right.$ po.d. $)$, when the median stage of maturation takes place

- $\quad$ Time period E ( $181^{\text {th }}-365^{\text {th }}$ po.d.), when the late stage of maturation takes place.

In order to find a representative (total) score for the cosmetic, the clinical, the ultrasonographical and the histopathological evaluation, at each time period, separate scores of some of the parameters of each category were added.

For cosmetic examination, intra-observer variability was first examined with the Mann-Whitney U test with the Bonferroni-Dunn method for controlling the type I error rate. Afterwards, the total score was calculated by averaging the separate scores given by the two observers.

For total clinical evaluation, the scores of skin thickening, scar width, hyperpigmentation, cross scar formation and inflammation were added. The values of the skin thickening and the scar width, before being added, were transformed to a 4scale score $(0-3)$, based on the $25^{\text {th }}, 50^{\text {th }}$ and $75^{\text {th }}$ percentile of the distribution of the total values.

For the ultrasonographic evaluation, four different tomographic sections (a, b, c, d) were examined at four sites along the scar in each incision, "a" being proximally and " $d$ " being distally to the hip joint. Wound area was calculated for each tomographic plan. The difference in the volumes between the tomographic sections was tested with Wilcoxon Signed Ranks Test, due to saliently violation of the normal distribution. In all the pairwise comparisons, we utilized the Bonferroni-Dunn correction to control the type I error. Furthermore, mean u/s wound area of each wound (derived by the four volume values taken in each wound) was transformed to a 4- scale score (0-3), based on the $25^{\text {th }}, 50^{\text {th }}$ and $75^{\text {th }}$ percentile of the distribution of the total values. Finally, in order to find if there is any correlation between $\mathrm{u} / \mathrm{s}$ estimated mean wound areas and various histopathological and clinical parameters, the Spearman's rank order correlation coefficient was used.

For total histological evaluation, the scores of edema, inflammatory reaction, tissue reaction around the suture material, thickness of the epidermis at the area of wound 
healing, epithelial gap and scar width were added. The values of the thickness of the epidermis at the area of wound healing, the epithelial gap and the scar width, before summation, were transformed to a 4 - scale score $(0-3)$, based on the $25^{\text {th }}, 50^{\text {th }}$ and $75^{\text {th }}$ percentile of the distribution of the total values. Tissue necrosis was not included, as there was no sign of it in any of the samples, and no further analysis was conducted. Collagen deposition, fibroblast presence, and angeiogenesis were also not included in the summation, as they did not differ between the techniques.

Furthermore, in order to compare the techniques over time, the total representative score of each technique was evaluated, on the $7^{\text {th }}, 14^{\text {th }}, 28^{\text {th }}, 180^{\text {th }}$ and $365^{\text {th }}$ po.d, by adding each time the cosmetic, clinical, ultrasonographical and histological total scores for the particular examination days. 


\section{RESULTS}

\section{Time required for each technique}

The time required for the performance of each skin closure technique is shown in Table 1 and Figure 1. The comparisons between techniques (Mann-Whitney test with Bonferroni correction) revealed that they all differed significantly from each other (all $\mathrm{P}<0.001)$.

Table 1. Time required for the performance of each technique

\begin{tabular}{|l|c|c|c|c|c|}
\hline \multicolumn{1}{|c|}{ Technique } & Percentile 25 & Median & Percentile 75 & Mean & $\begin{array}{c}\text { Standard } \\
\text { Deviation }\end{array}$ \\
\hline staples & $18^{\prime \prime}$ & $21^{\prime \prime}$ & 25 & $21^{\prime \prime}$ & $5^{\prime \prime}$ \\
\hline glue & $1^{\prime} 45^{\prime \prime}$ & $2^{\prime} 16^{\prime \prime}$ & $2^{\prime} 32^{\prime \prime}$ & $2^{\prime} 12^{\prime \prime}$ & $3^{\prime \prime}$ \\
\hline intradermal B & $15^{\prime} 32^{\prime \prime}$ & $15^{\prime} 37^{\prime \prime}$ & $16^{\prime} 55^{\prime \prime}$ & $16^{\prime} 13^{\prime \prime}$ & $1^{\prime} 30^{\prime \prime}$ \\
\hline intradermal C & $13^{\prime} 39^{\prime \prime}$ & $13^{\prime} 48^{\prime \prime}$ & $14^{\prime} 05^{\prime \prime}$ & $13^{\prime} 33^{\prime \prime}$ & $44^{\prime \prime}$ \\
\hline
\end{tabular}

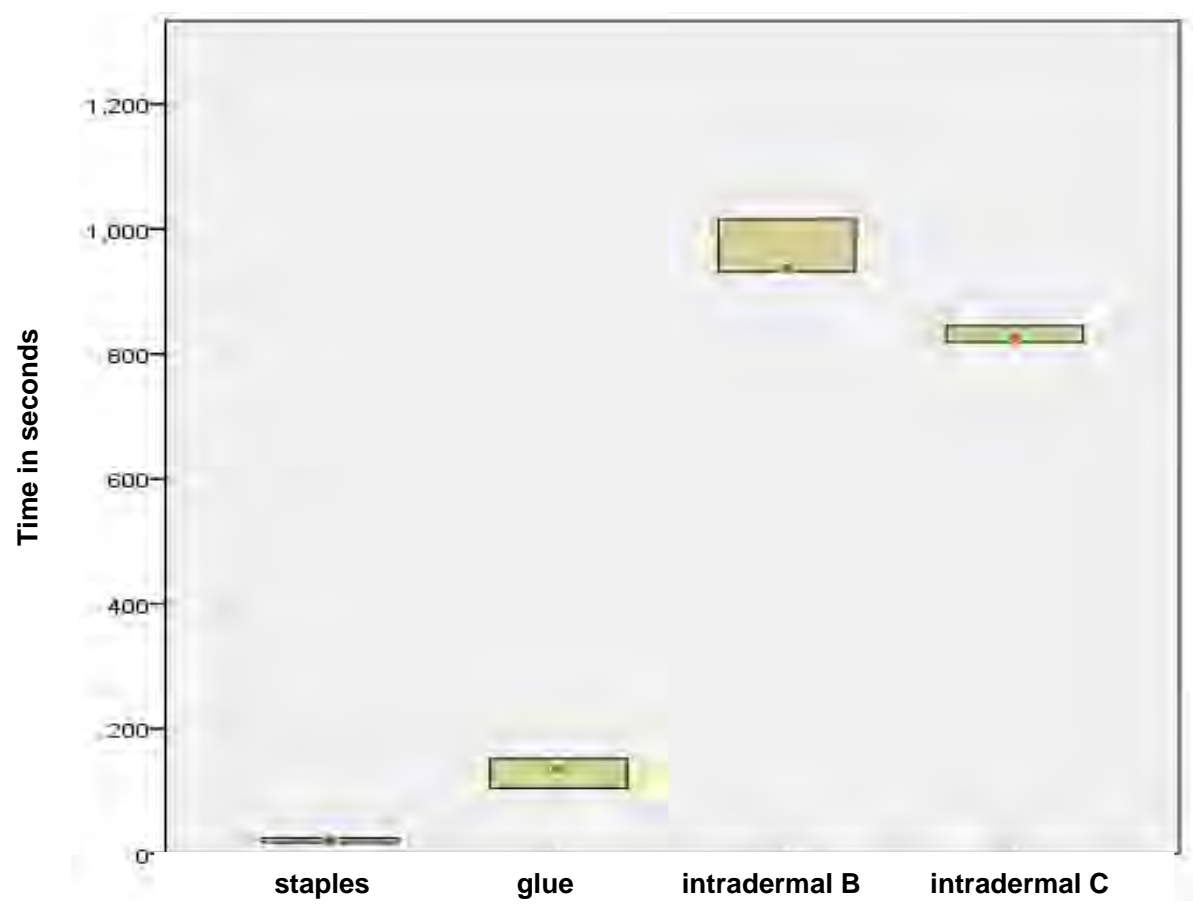

Figure 1. Median (red dot) and interquartile range (column) of the time required (in seconds) for the performance of each technique

\section{Cosmetic evaluation}

\section{Comparison of cosmetic evaluation among assessors}

Generally, there was no statistical difference between the scores of the two assessors during the evaluation of the cosmetic appearance of the wounds $(\mathrm{P}=0.914)$. 
However, there was a significant difference between the assessors' cosmetic evaluation scores $(\mathrm{P}<0.001)$ in Period $\mathrm{A}\left(1^{\text {st }}-8^{\text {th }}\right.$ day), with the median score given from the first assessor being 4 (3-4) and from the second assessor 5 (4-5). In period B $\left(9^{\text {th }}-21^{\text {st }}\right.$ po.d. $)$, a significant difference between the scores of the assessors was also observed $(\mathrm{P}<0.001)$; the median score for the first assessor was $3(2.5-3.5)$ and for the second assessor was 2 (1-3). In periods C, D and E no statistical difference was observed $(\mathrm{P}>0.166)$. The median score given by each assessor in each time period is shown in Figure 2.

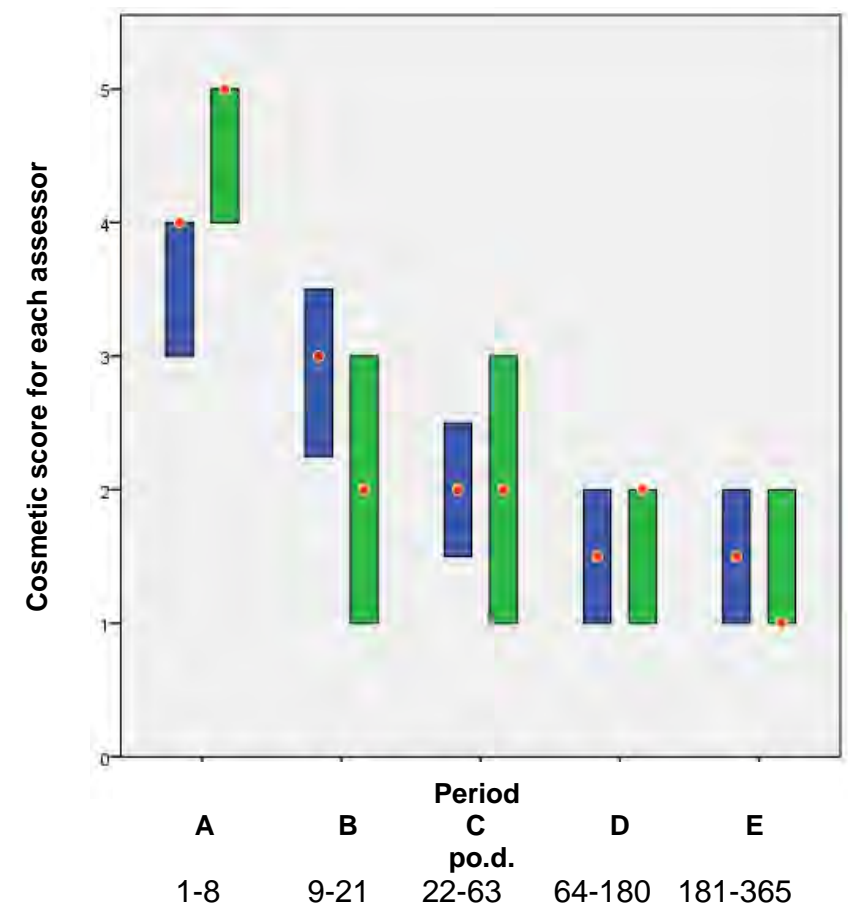

Figure 2. Median score (red dot) and interquartile range (column) of the cosmetic score for each assessor (blue or green columns) in each time period.

Furthermore, no statistical difference was observed between the assessors' median score regarding the cosmetic appearance of each technique. As regards staples, the median score for the first assessor was 2 (1-2.5), and for the second assessor was 2 (1$2)$. As regards glue, the median score for the first assessor was 2 (1.5-2.5), and for the second assessor was 2 (1-3). As regards intradermal B, the median score for the first assessor was 1.5 (1-2), and the second assessor's median score was 2 (1-2). As regards intradermal $\mathrm{C}$, the median score for the first assessor was 1.5 (1-2), and the second assessor's was 1 (1-2). The median score for each assessor for each technique is presented in Figure 3. 


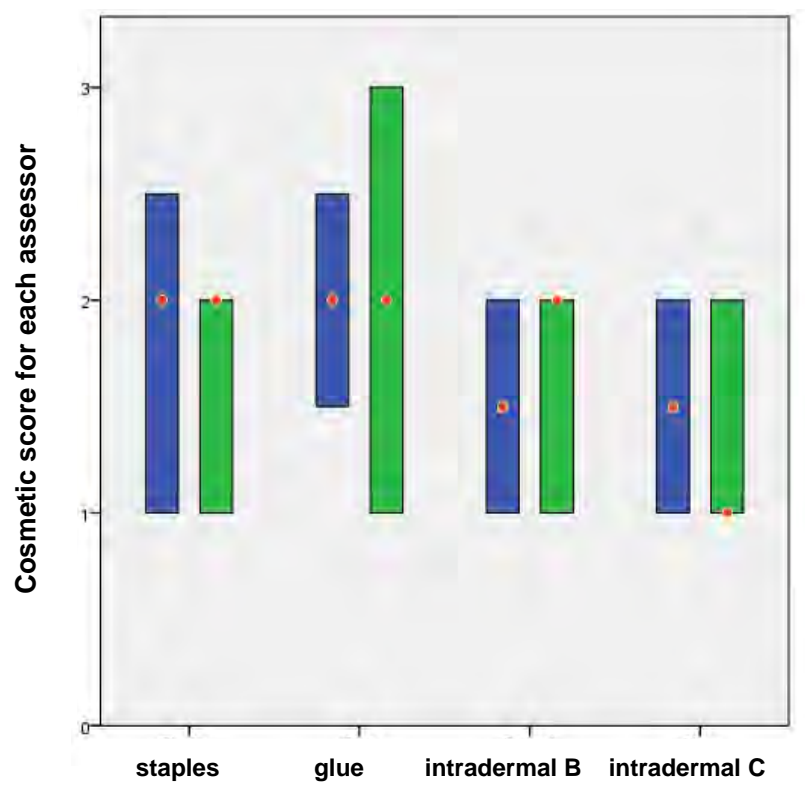

Figure 3. Median (red dot) and interquartile range (column) of the cosmetic score for each assessor (blue or green columns) for each technique.

\section{Total cosmetic evaluation}

In Period $\mathrm{A}$, the median score of the total cosmetic evaluation (average score) was 4 (4-5) as regards staples, 4.8 (4-5) as regards glue, 4 (4-4.5) as regards intradermal B and 4 (3.3-4.5) as regards the intradermal C. In period B, it was 2 (1.5-3) as regards staples, 3 (2.5 -4) as regards glue, 2.8 (2-3) as regards intradermal B and 2 (1-3) as regards intradermal C. In period C, it was 2 (2-2.8) as regards staples, 3 (2-3) as regards glue, $2(1.8-3)$ as regards intradermal $\mathrm{B}$ and $1.8(1.2)$ as regards intradermal C. No statistical difference was observed between the techniques during these time periods. In period D, the median score was 2 (1-2) as regards staples, 2 (1-2.5) as regards glue, 1 (1-2) as regards intradermal B and 1 (1-2) as regards intradermal C. Statistically significant difference was observed between glue and intradermal $\mathrm{C}(\mathrm{P}=$ 0.006). In period $\mathrm{E}$ the median score was 1.5 (1-2) as regards staples, $2(1.3-2)$ as regards glue, 1 (1-2) as regards intradermal $\mathrm{B}$ and 1.3 (1-2) as regards intradermal C, and statistical differences were observed between glue and intradermal B $(\mathrm{P}<0.001)$, and between glue and intradermal $\mathrm{C}(\mathrm{P}<0.001)$. The median score for each technique in each time period is presented in figure 4. 


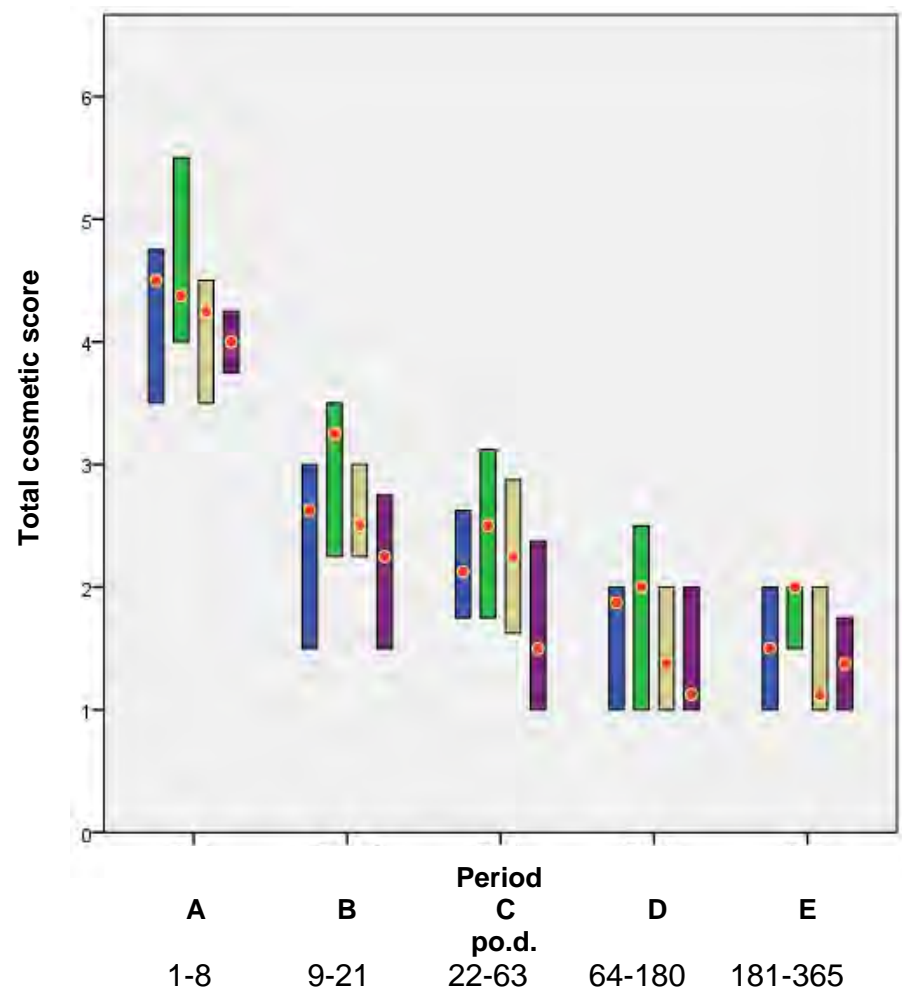

Figure 4. Median score (red dot) and interquartile range (column) of the total cosmetic score for each technique (blue: staples, green: glue, gray: intradermal B and purple: intradermal C) in each time period.

\section{Clinical evaluation}

The healing process progressed normally without severe complications in all animals and with all techniques. Photographs of the incisions taken at various times postoperatively are presented in the Addendums 1a and $1 \mathrm{~b}$.

\section{Skin thickening}

Skin thickening at the wound area was observed from the $1^{\text {st }}$ po.d. with all techniques and in all animals, due to the swelling of the tissues. The thickness remained increased until the $3^{\text {rd }}$ to $6^{\text {th }}$ po.d. with all techniques, while the largest skin thickening with all techniques was observed on the $1^{\text {st }}$ po.d. Skin thickness decreased and subsided completely on the $70^{\text {th }}$ po.d. with all techniques. The median skin thickening for each technique at each time point is presented in figure 5. 


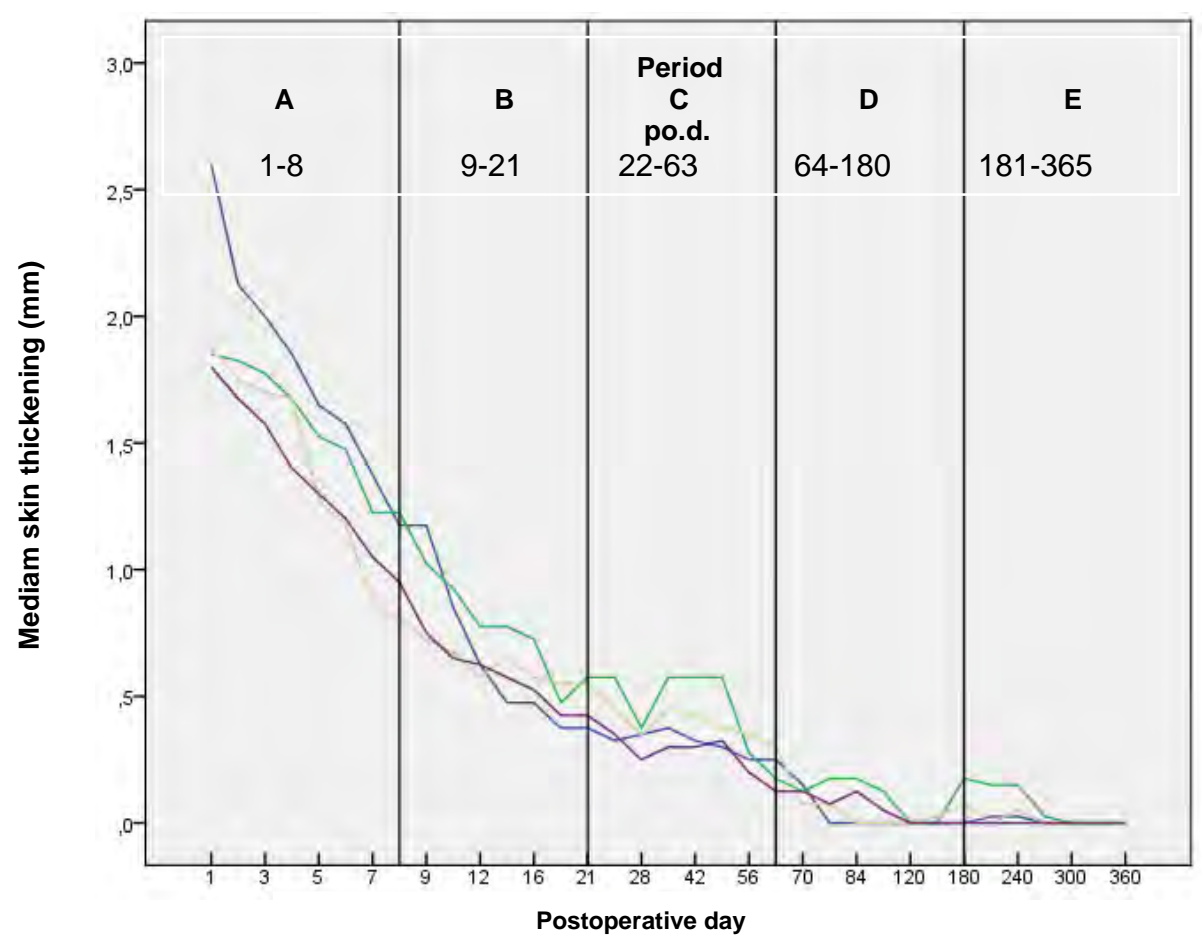

Figure 5. Median skin thickening (in $\mathrm{mm}$ ) with each technique (blue: staples, green: glue, gray: intradermal B and purple: intradermal C) at each time point.

In period A, the median skin thickening (in mm) was 1.75 (1.28-2.63) as regards staples, 1.65 (0.9-2.05) as regards glue, 1.53 (1.03-1.85) as regards intradermal B and $1.4(1-1.7)$ as regards intradermal $C$, as regards staples being significantly different from intradermal B $(\mathrm{P}<0.001)$ and from intradermal $\mathrm{C}(\mathrm{P}<0.001)$. The maximum thickening $(4.35 \mathrm{~mm})$ was noticed with staples on the $8^{\text {th }}$ po.d. In period $\mathrm{B}$, the median skin thickening (in mm) was $0.68(0.40-1.25)$ as regards staples, $0.6(0.25-1.50)$ as regards glue, $0.65(0.35-0.75)$ as regards intradermal $\mathrm{B}$ and $0.58(0.30-0.10)$ as regards intradermal C, whilst no statistical differences were observed between the techniques. In period $\mathrm{C}$, the median skin thickening (in mm) was $0.30(0.10-0.50)$ as regards staples, $0.55(0-0.90)$ as regards glue, $0.38(0.05-0.75)$ as regards intradermal $\mathrm{B}$, and $0.30(0.10-0.50)$ as regards intradermal $C$, with no significant differences observed between the techniques. No skin thickening was noticed in periods D and $\mathrm{E}$ with any technique. The median skin thickening for each technique in each time period is presented in figure 6 . 


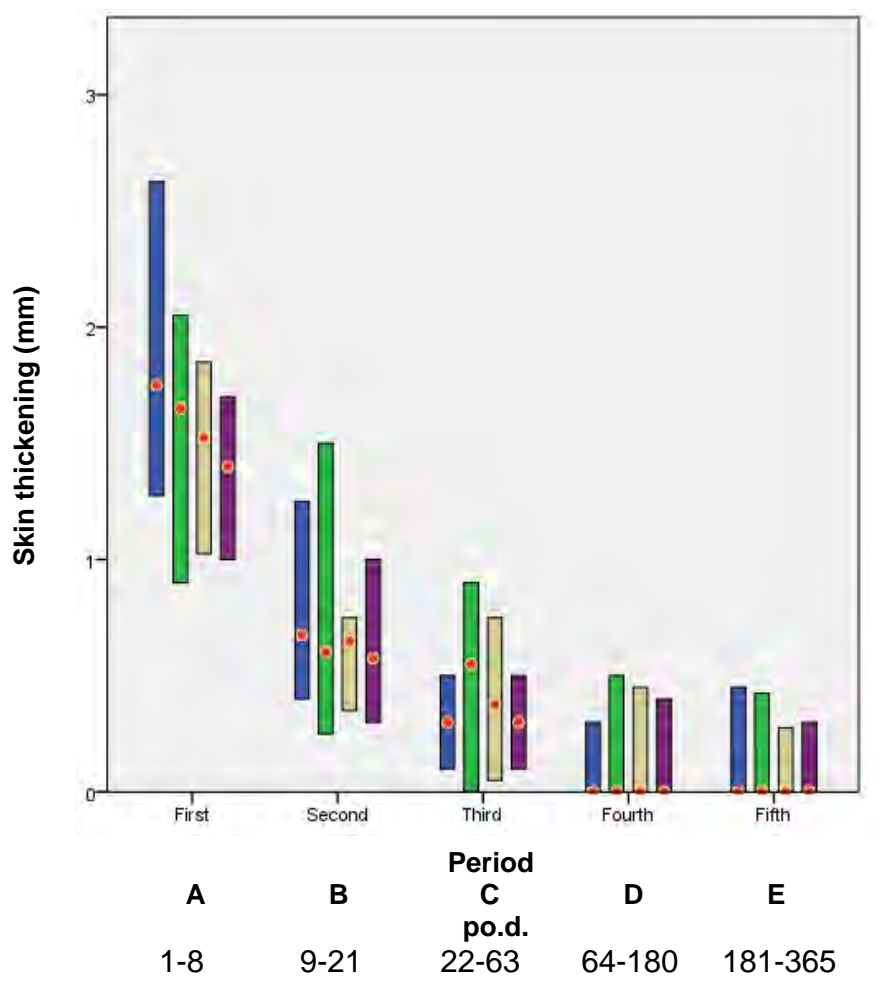

Figure 6. Median (red dot) and interquartile range (column) of skin thickening (in $\mathrm{mm}$ ) for each technique (blue: staples, green: glue, gray: intradermal B and purple: intradermal C) in each time period.

With the continuous intradermal suture pattern with burying of the knot, skin thickening was observed in the areas where the initial and the final knot were buried. Swelling appeared from the $5^{\text {th }}$ po.d. and in some animals remained as mild swelling until the $42^{\text {nd }}$ po.d.

\section{Erythema}

Erythema was observed in all animals with all techniques during period A. In period $\underline{B}$, it remained in some animals with all techniques but intradermal $\mathrm{B}$, while in period $\underline{\mathrm{C}}$, it was observed in one incision only closed with staples and in one closed with glue (Figure 7).

In period $\mathrm{A}$, the median erythema (in $\mathrm{mm})$ was $0.4(0-1.19$, max 4$)$ as regards staples, $0.31(0-1.49$, max 4.96) as regards glue, $0.3(0-0.7$, max 3.75) as regards intradermal B and 0.32 (0-0.6, max 3.9) as regards intradermal C, with no significant differences observed between techniques. The median skin erythema (in mm) for each technique in period $\mathrm{A}$ is presented in figure 8.

In periods B, C and D erythema was present only in some wounds. In period B, the maximum erythema (in $\mathrm{mm}$ ) was 5.14, 2.08, 0 and 1.06 as regards staples, glue, intradermal $\mathrm{B}$, and intradermal $\mathrm{C}$, respectively. In period $\mathrm{C}$, the maximum erythema (in $\mathrm{mm}$ ) was 2.07, 3.7, 0 and 0 as regards staples, glue, intradermal B, and intradermal C, respectively. Finally, in periods D and E, no erythema was observed. 


\section{Scar width}

The scar width decreased gradually until the $21^{\text {st }}$ po.d. with all techniques but glue, with which the scar width increased. Then, up to the $180^{\text {th }}$ po.d., it remained stable with all techniques but glue, with which it decreased. Until the $365^{\text {th }}$ po.d., the scar width decreased with all techniques (Figure 9). The median scar width with each technique at each time point is presented in figure 10.
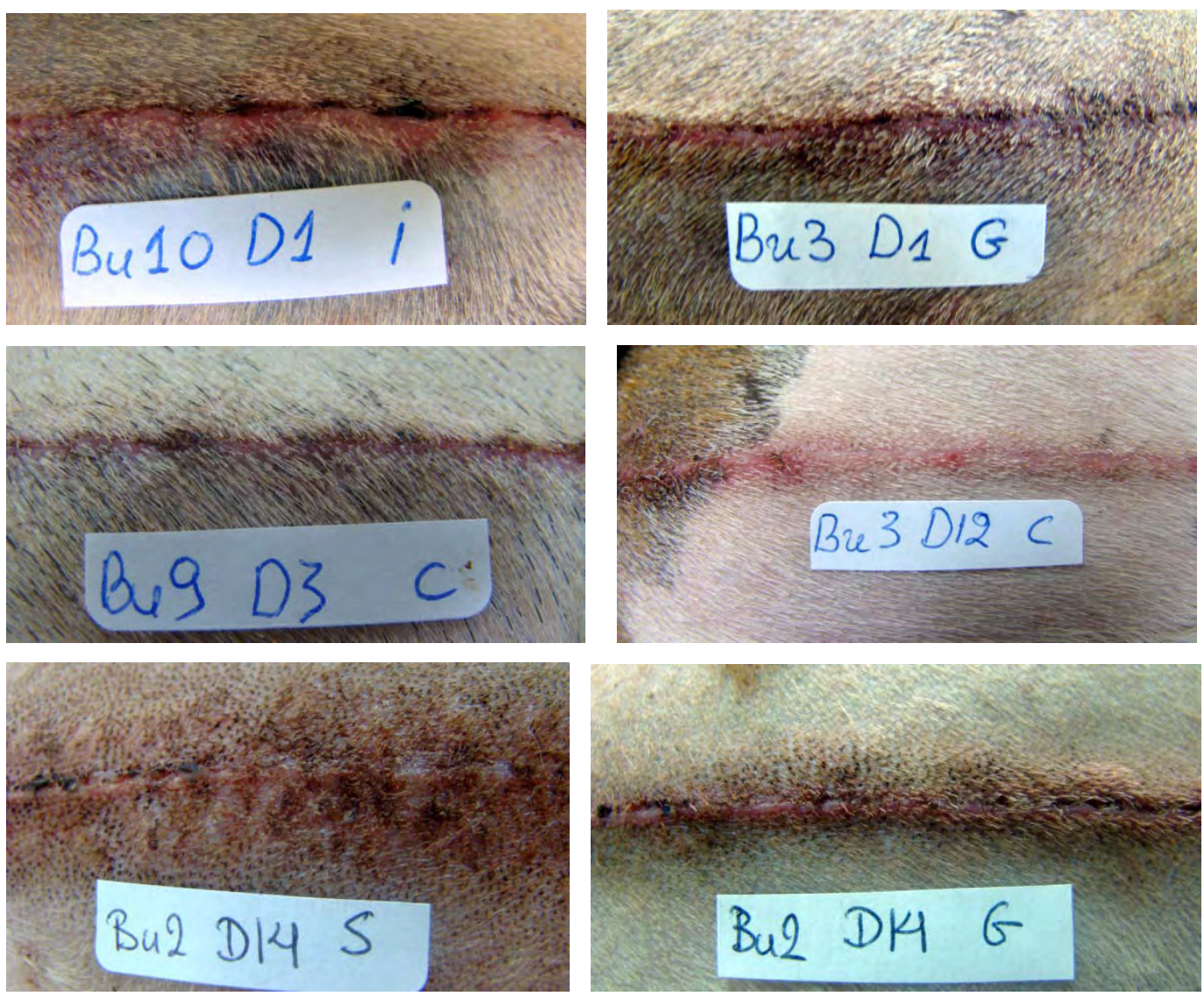

Figure 7. Erythema at wound area up to the $14^{\text {th }}$ po.d. (i: intradermal B, G: glue, c: intradermal C, s: staples) 


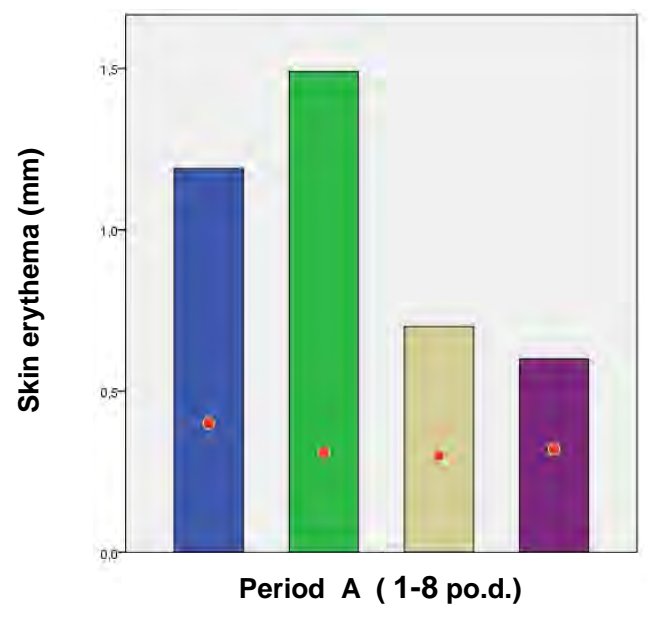

Figure 8. Median (red dot) and interquartile range (column) of skin erythema (in mm) for each technique (blue: staples, green: glue, gray: intradermal B and purple: intradermal C) in period A.
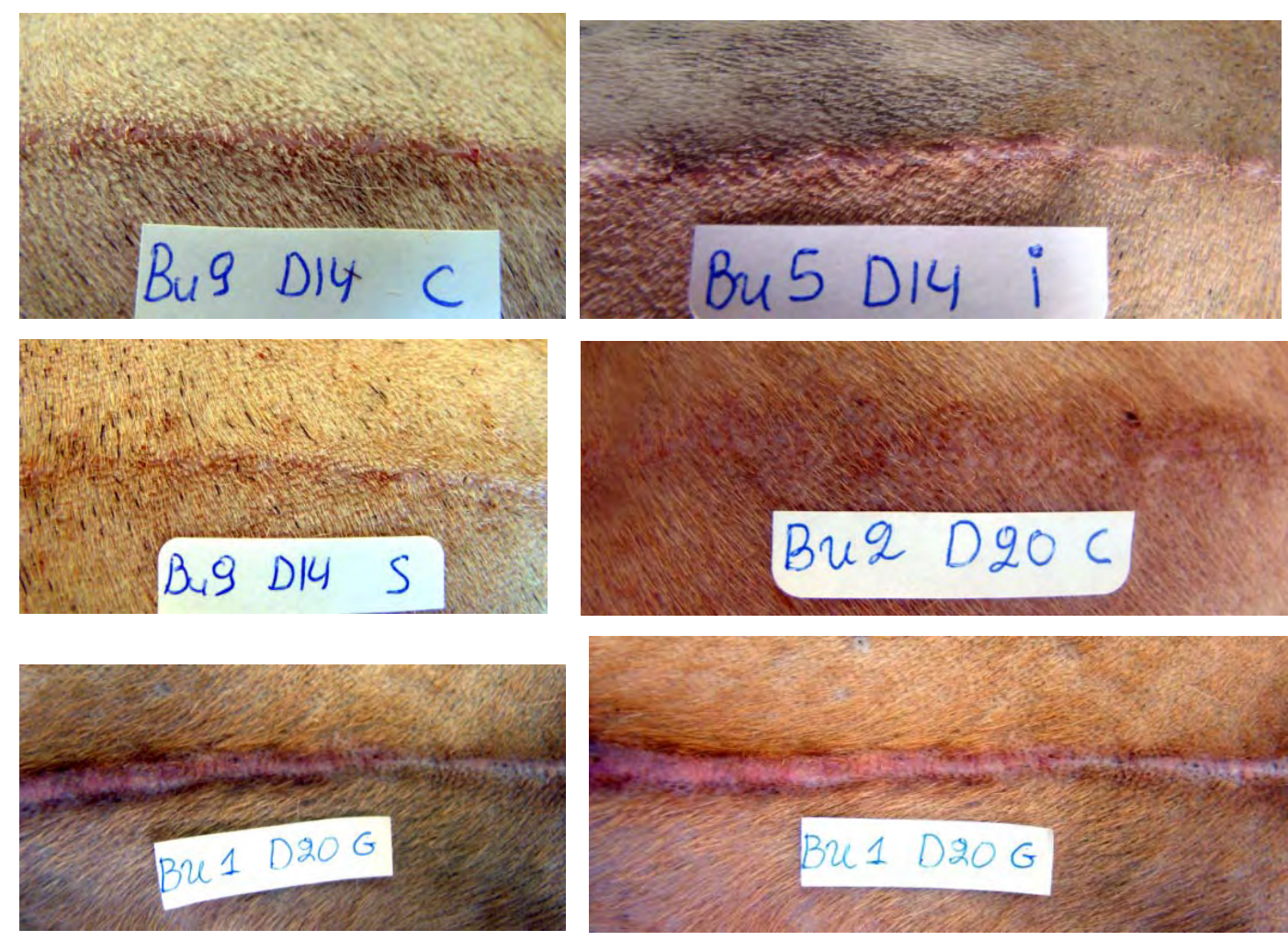

Figure 9. Scar width at wound area (i: intradermal B, G: glue, c: intradermal C, s: staples) 

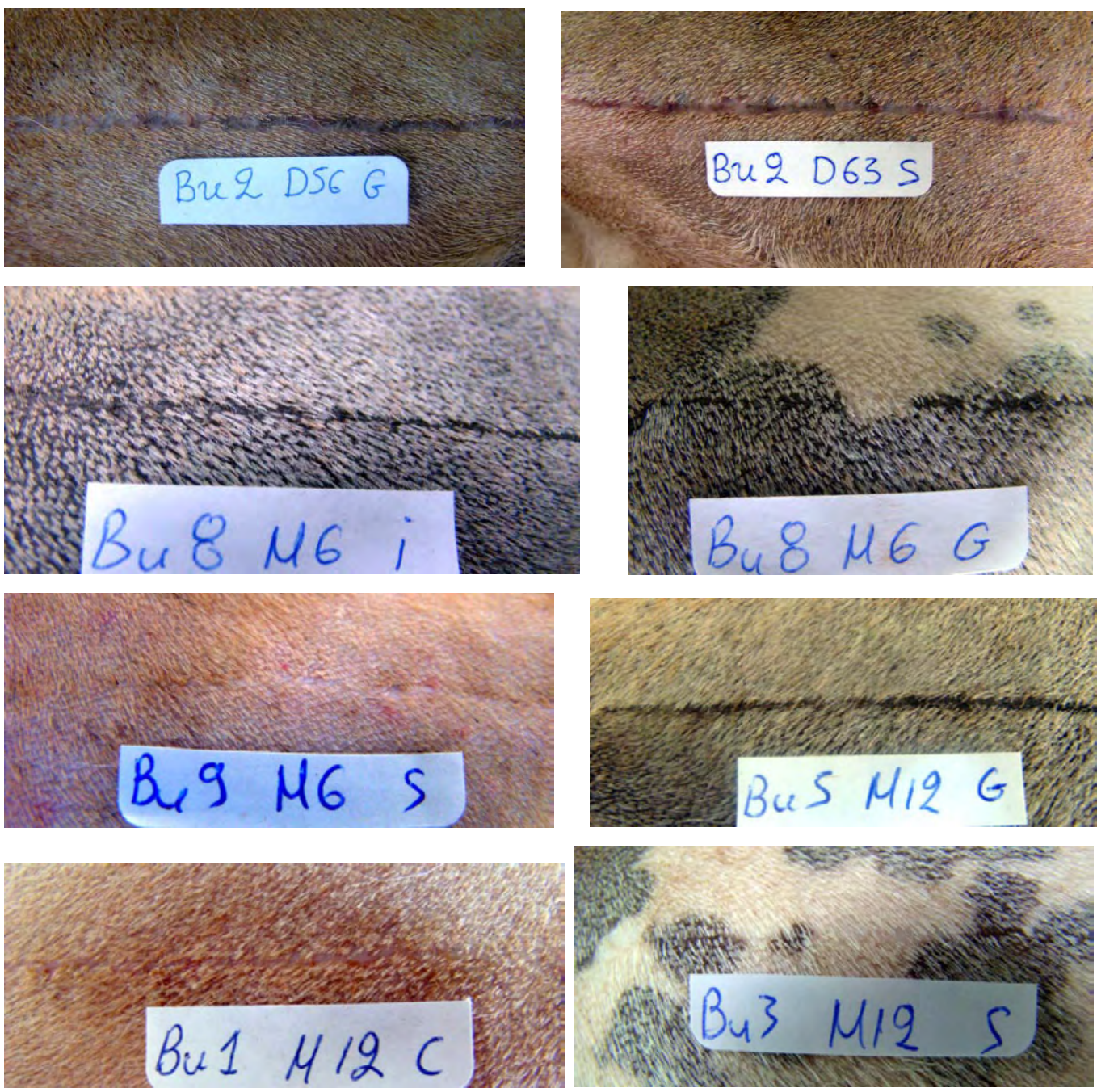

Figure 9. (continued) (c: intradermal C, s: staples) 


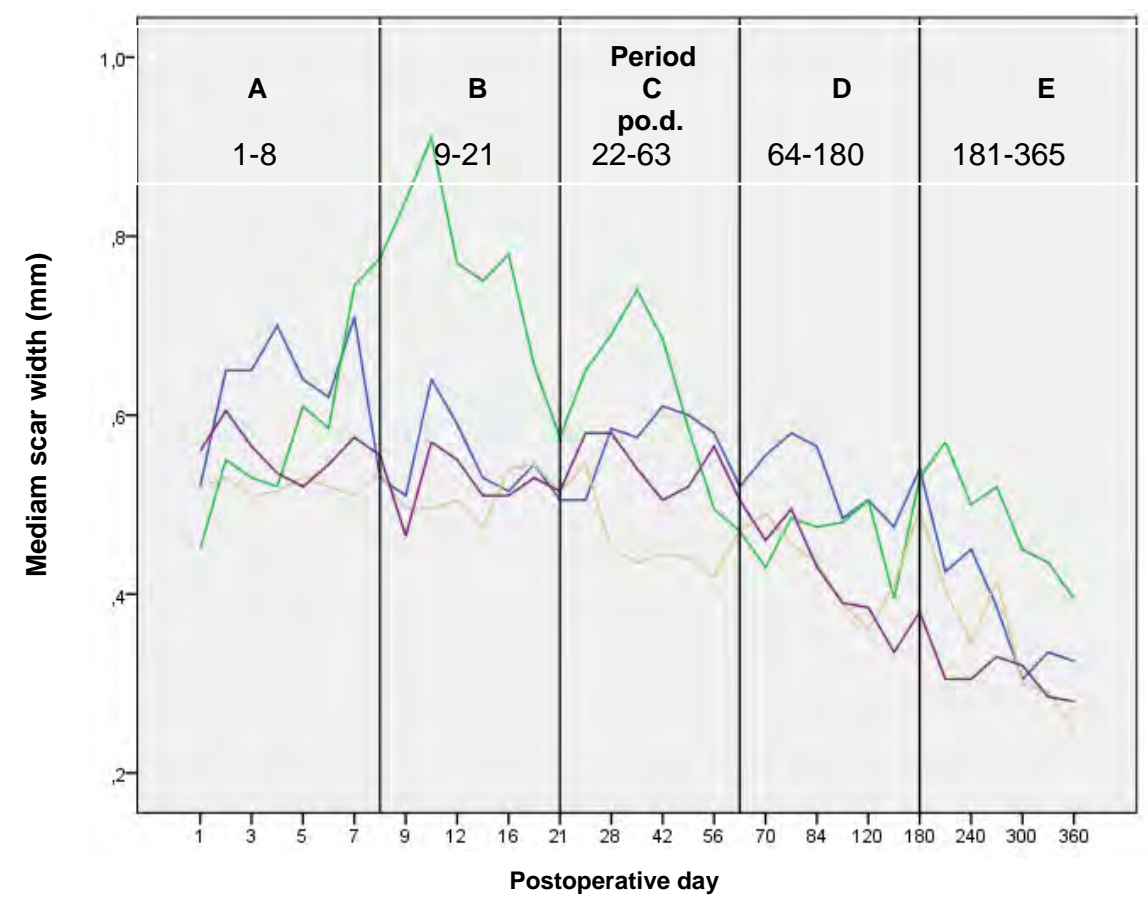

Figure 10. Median scar width (in $\mathrm{mm}$ ) for each technique (blue: staples, green: glue, gray: intradermal B and purple: intradermal C) at each time point.

In period $\mathrm{A}$, the median scar width (in $\mathrm{mm}$ ) was $0.64(0.44-1)$ as regards staples, $0.57(0.42-0.84)$ as regards glue, $0.52(0.43-0.6)$ as regards intradermal $\mathrm{B}$ and 0.56 (0.47-0.67) as regards intradermal C, without any significant differences between the techniques.

In period $\mathrm{B}$, the median scar width (in $\mathrm{mm}$ ) was $0.53(0.43-0.8)$ as regards staples, $0.77(0.38-0.93)$ as regards glue, $0.51(0.44-0.6)$ as regards intradermal $\mathrm{B}$ and 0.53 (0.45-0.6) as regards intradermal C. Glue was significantly different from intradermal $\mathrm{B}(\mathrm{P}=0.001)$ and intradermal $\mathrm{C}(\mathrm{P}<0.001)$.

In period $\mathrm{C}$, the median scar width (in $\mathrm{mm}$ ) was 0.51 (0.32-0.87 as regards staples, $0.55(0.4-0.84)$ as regards glue, $0.45(0.37-0.6)$ as regards intradermal $\mathrm{B}$ and 0.56 (0.41-0.66) as regards intradermal $C$. Glue was significantly different from intradermal $\mathrm{B}(\mathrm{P}=0.008)$.

In period D, the median scar width (in mm) was $0.49(0.25-0.77)$ as regards staples, $0.47(0.30-0.63)$ as regards glue, $0.42(0.29-0.56)$ as regards intradermal $\mathrm{B}$ and 0.41 (0.27-0.57) as regards intradermal C. No statistically significant differences were revealed between the techniques.

In period E, the median scar width (in $\mathrm{mm}$ ) was 0.38 (0.25-0.63) as regards staples, $0.48(0.32-0.67)$ as regards glue, $0.35(0.23-0.48)$ as regards intradermal $\mathrm{B}$ and 0.30 (0.24-0.48) as regards intradermal C. Glue was significantly different from intradermal B $(\mathrm{P}<0.001)$ and intradermal $C(\mathrm{P}<0.001)$. The median scar width (in $\mathrm{mm}$ ) for each technique in each period is presented in figure 11. 


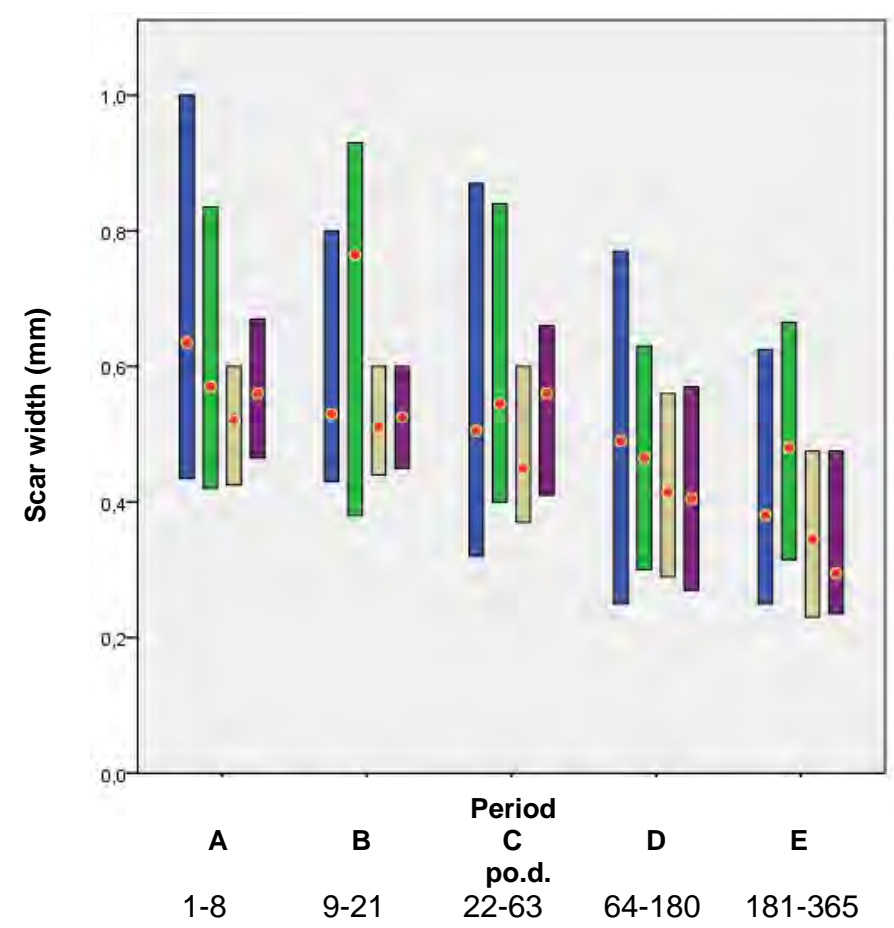

Figure 11. Median (red dot) and interquartile range (column) of scar width (in mm) for each technique (blue: staples, green: glue, gray: intradermal B and purple: intradermal C) in each period.

\section{Inflammation and Abscessation}

Inflammation was observed in six animals, and in all but one wound was evaluated with score 1, i.e. mild inflammation. In Beagle No 1, inflammation was observed the first po.d. at the wounds closed with glue and staples, in Beagle No 2 was observed the $10^{\text {th }}$ po.d at the wounds closed with glue and staples, and in Beagle No 5 the first two po.d. at the wounds closed with intradermal B and with glue. In Beagle No 9 it was observed from the $21^{\text {st }}$ to the $35^{\text {th }}$ po.d at the wound closed with intradermal $B$ and on the $28^{\text {th }}$ po.d at the wound closed with staples. In Beagle No10 it was observed from the $18^{\text {th }}$ to the $77^{\text {th }}$ po.d at the wound closed with the intradermal $B$ and on the $63^{\text {rd }}$ po.d. at the wound closed with glue. In intradermal $C$ there was only one case of mild inflammation in Beagle No 7 on the $16^{\text {th }}$ po.d. In only one case a microabscess was noticed in Beagle No 9 on the $28^{\text {th }}$ po.d at the wound sutured with intradermal B. (Figure 12) The number of inflammation cases observed for each technique is shown in Table 2.

Table 2. Cases of inflammation for each technique

\begin{tabular}{|c|c|c|c|c|c|c|}
\hline & \multirow[b]{2}{*}{ score } & \multicolumn{4}{|c|}{ TECHNIQUE } & \multirow[b]{2}{*}{ Total } \\
\hline & & Staples & Glue & intradermal B & $\begin{array}{c}\text { intradermal } \\
\text { C }\end{array}$ & \\
\hline \multirow{2}{*}{$\begin{array}{l}\text { Cases of } \\
\text { inflammation }\end{array}$} & 1 & 5 & 9 & 14 & 1 & 29 \\
\hline & 2 & 0 & 0 & 1 & 0 & 1 \\
\hline \multicolumn{2}{|l|}{ Total } & $5(3)^{*}$ & $9(4)$ & $15(3)$ & $1(1)$ & 30 \\
\hline
\end{tabular}

*In brackets is the number of animals that showed inflammation. 

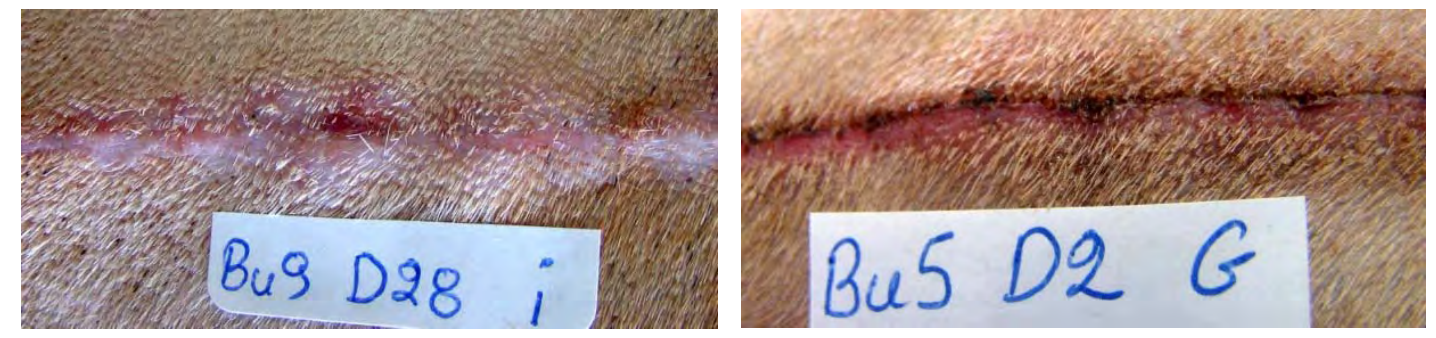

Figure 12. Inflammation of the wound area (G: glue, i: intradermal B).

\section{Exudate}

No exudate was observed in any incision of any animal.

\section{Comedones}

Comedones were observed only on three occasions in two animals. One was observed on the $77^{\text {th }}$ po.d. in an incision closed by staples and the other two in an incision closed by intradermal with burying of the knot on the $35^{\text {th }}$ and $42^{\text {nd }}$ po.d.

\section{Hyperpigmentation at the wound area}

Hyperpigmentation was observed in all wounds with all the techniques from the $28^{\text {th }}$ (or $35^{\text {th }}$ ) until the $365^{\text {th }}$ po.d. The intensity of the hyperpigmentation differed according to the technique and to the period of evaluation. Wounds with intense hyperpigmentation initially and with mild hyperpigmentation afterwards were observed with staples. From the $12^{\text {th }}$ po.d, hyperpigmentation was observed in the cross scaring formations, too. As regards glue, the wounds had intense hyperpigmentation that was maintained for a longer period, whilst as regards intradermal B and intradermal $\mathrm{C}$ hyperpigmentation was absent or mild and remained for a shorter period (Figure 13).

In period $C$, the median wound hyperpigmentation score was $2(0-3)$ as regards staples, $1(1-2)$ as regards glue, $1(0-1)$ as regards intradermal $B$ and $0(0-1)$ as regards intradermal C. Staples differed significantly from intradermal $\mathrm{B}(\mathrm{P}=0.003)$ and intradermal $\mathrm{C}(\mathrm{P}<0.001)$, and glue differed significantly from intradermal $\mathrm{B}(\mathrm{P}=0.009)$ and intradermal $\mathrm{C}(\mathrm{P}<0.001)$.

In period D, the median wound pigmentation score was 1 (1-3) as regards staples, 1 $(0-2)$ as regards glue, $0(0-1)$ as regards intradermal $B$ and $0(0-1)$ as regards intradermal C. Staples differed significantly from intradermal $B(P<0.001)$ and intradermal $\mathrm{C}(\mathrm{P}<0.001)$. Glue was also significantly different from intradermal $\mathrm{B}$ $(\mathrm{P}=0.002)$ and intradermal $\mathrm{C}(\mathrm{P}<0.001)$.

In period $\mathrm{E}$, the median wound pigmentation score was $1(0-2)$ as regards staples, 1 (1-2) as regards glue, $0.5(0-1)$ as regards intradermal $\mathrm{B}$ and 1 (1-1) as regards intradermal C. Glue was significantly different from intradermal $\mathrm{B}(\mathrm{P}=0.002)$ and intradermal $\mathrm{C}(\mathrm{P}=0.005)$.

The median wound pigmentation score for each technique in each period is presented in figure 14. 

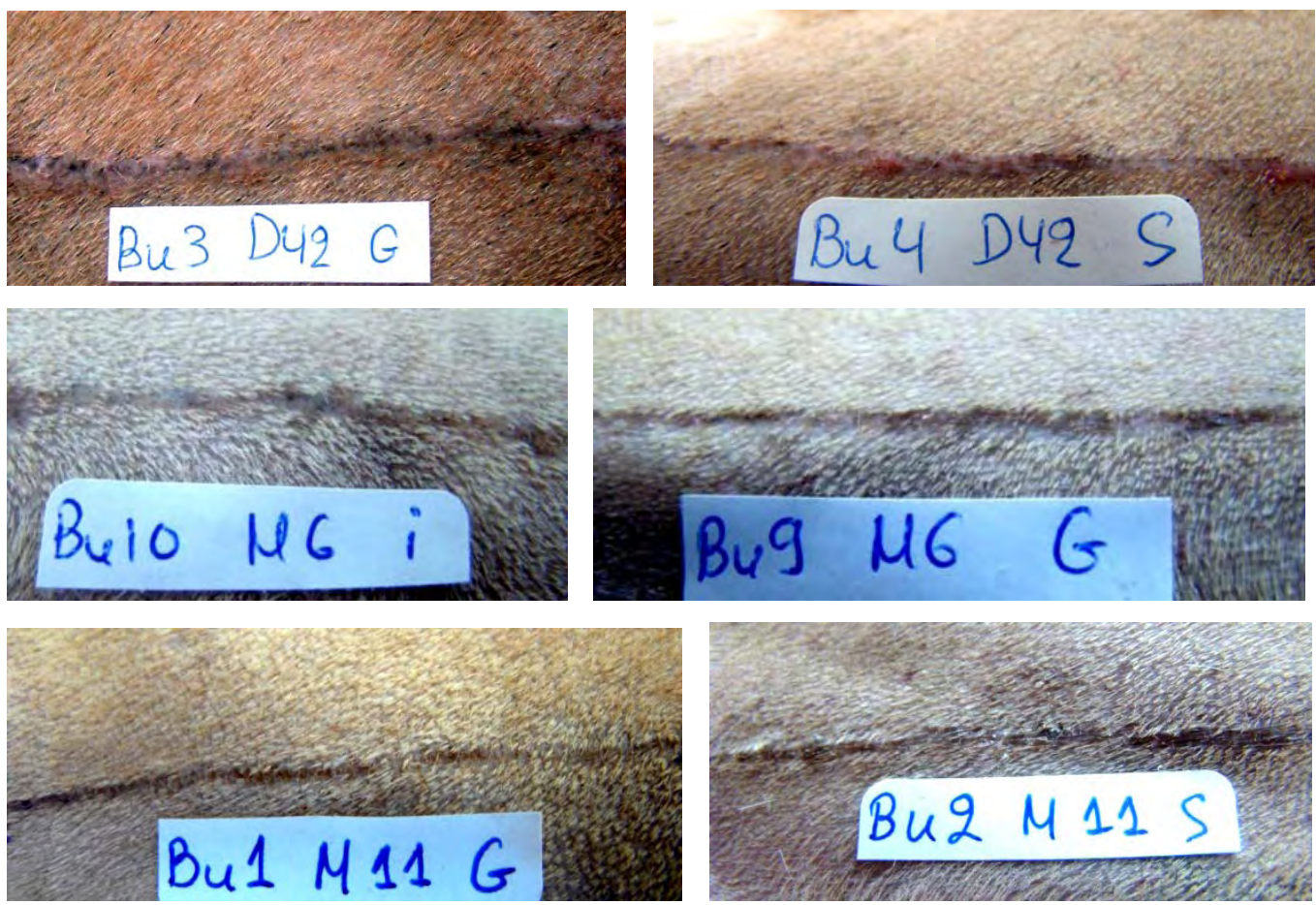

Figure 13. Pigmentation at wound area (G: glue, S: staples, i: intradermal B).

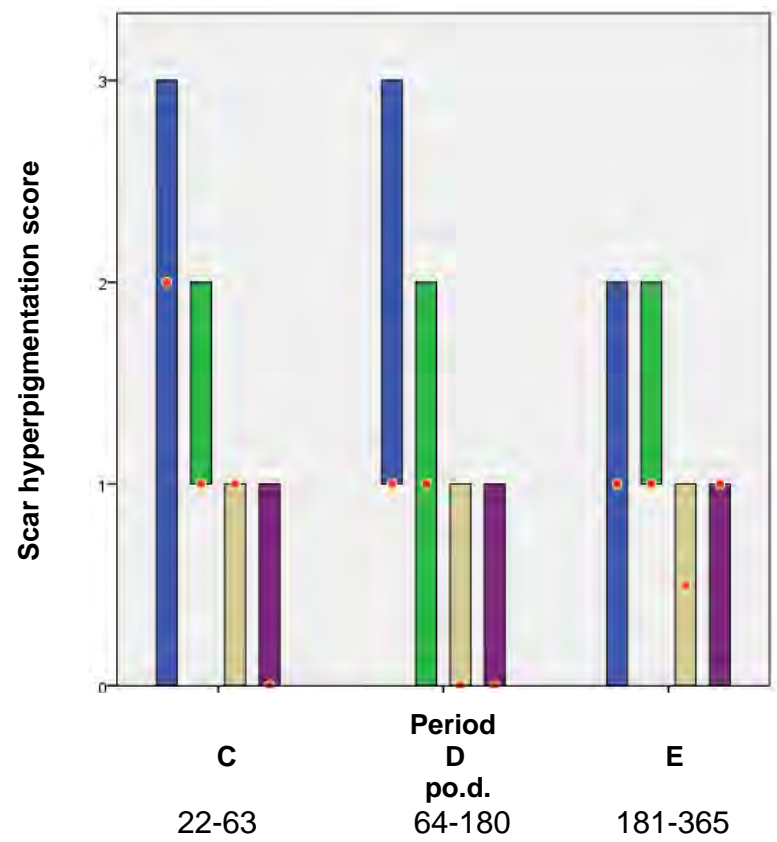

Figure 14. Median (red dot) and interquartile range (column) of scar hyperpigmentation score for each technique (blue: staples, green: glue, gray: intradermal B and purple: intradermal C) in each period. 


\section{Staple loss}

During the first 10 postoperative days, only four staples were removed from the skin by the animals. One staple was removed the $1^{\text {st }}$ po.d. by one animal, two on the $4^{\text {th }}$ po.d. by another, and one on the $7^{\text {th }}$ po.d. by a third animal.

\section{Wound dehiscence}

Only one case of wound dehiscence was observed in an incision closed with staples. It occurred on the $12^{\text {th }}$ po.d., two days after staple removal and its length was 0.8 centimeters. The defect closed four days afterwards by second intention.

\section{Cross scaring formation}

Cross scaring formations were observed in all scars as regards staples. Until the $16^{\text {th }}$ po. day, the formations were observed in all scars, while on the $21^{\text {st }}$ po.d. were observed in $9 / 10$ scars. There were noticed in $7 / 10$ scars on the $28^{\text {th }}$ day, in $5 / 10$ on the $35^{\text {th }}$ day, in $3 / 10$ on the $42^{\text {nd }}$ day and in $1 / 10$ scars on the $70^{\text {th }}$ po.d. (Figure 15 ).

The frequency and the relative frequency of the cross scaring score is presented in Figure 16 and Table 3.
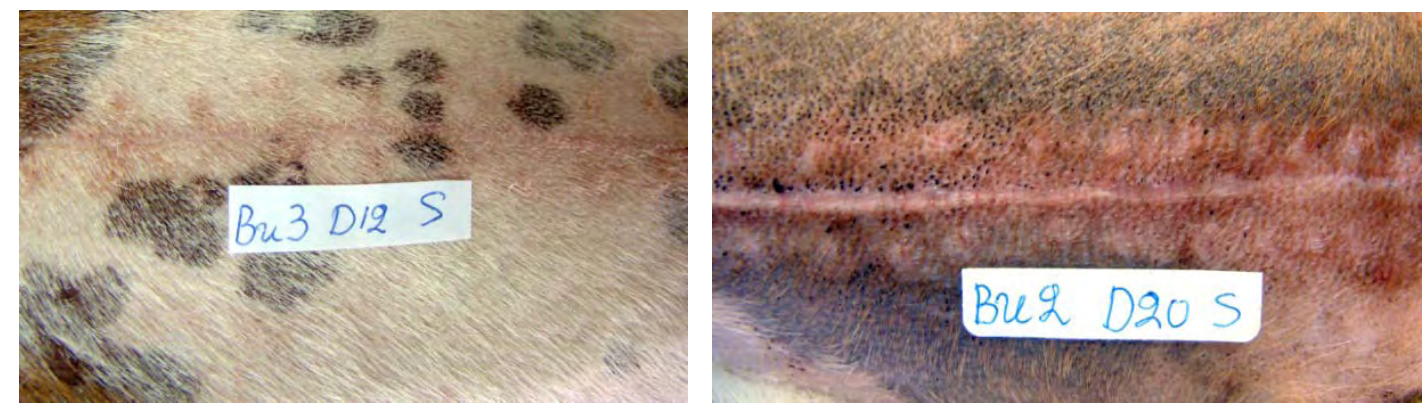

Figure 15. Cross scar formation at wound area (s: staples).

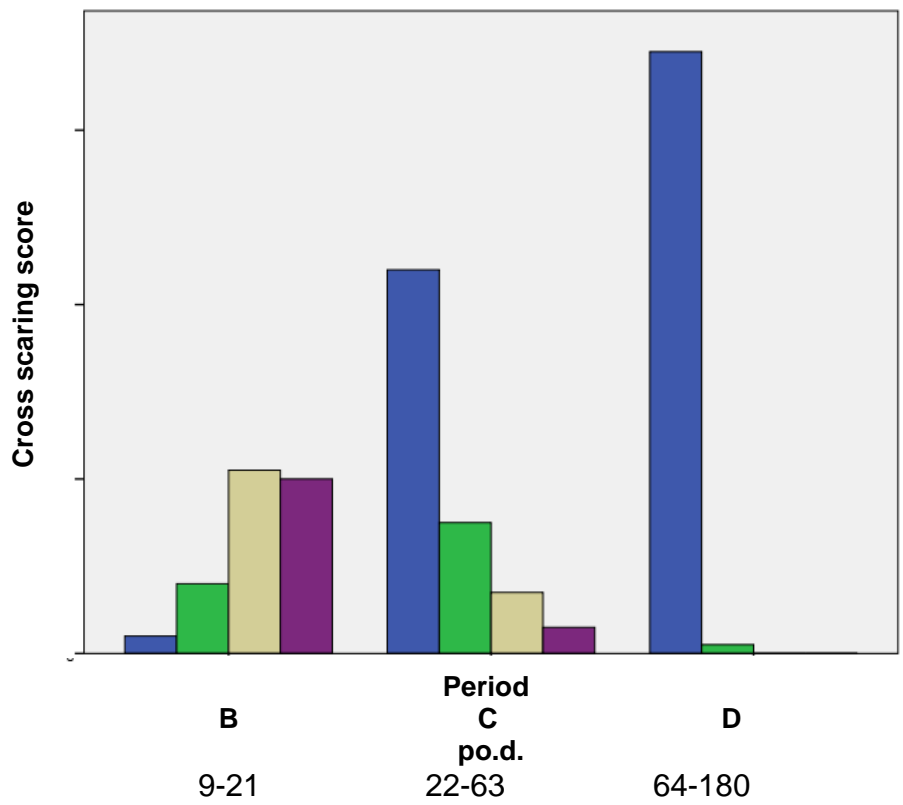

Figure 16. Frequency (column) of each cross scaring score (blue: staples, green: glue, gray: intradermal B and purple: intradermal C) in each period. 
Table 3. Frequency and relative frequency of the cross scaring score as regards staples in periods $\mathrm{B}, \mathrm{C}$, and $\mathrm{D}$

\begin{tabular}{|c|c|c|c|}
\hline Period & Score & Frequency & Percent \\
\hline \multirow{4}{*}{ Period B } & 0 & 2 & .7 \\
\cline { 2 - 4 } & 1 & 8 & 2.9 \\
\cline { 2 - 4 } & 2 & 21 & 7.5 \\
\cline { 2 - 4 } & 3 & 20 & 7.1 \\
\cline { 2 - 4 } & Total & 51 & 18.2 \\
\hline \multirow{5}{*}{ Period C } & 0 & 44 & 15.7 \\
\cline { 2 - 4 } & 1 & 15 & 5.4 \\
\cline { 2 - 4 } & 2 & 7 & 2.5 \\
\cline { 2 - 4 } & 3 & 3 & 1.1 \\
\cline { 2 - 4 } & Total & 69 & 24.6 \\
\hline \multirow{5}{*}{ Period D } & 0 & 69 & 24.6 \\
\cline { 2 - 4 } & 1 & 1 & .4 \\
\cline { 2 - 4 } & Total & 70 & 25 \\
\hline
\end{tabular}

\section{Total Clinical Evaluation}

In period A, the median score of the total clinical evaluation was 5 (3-6) as regards staples, 5 (4-6) as regards glue, 4 (4-5) as regards intradermal B and 4 (4-5) as regards intradermal $\mathrm{C}$, with no significant differences observed between the techniques.

In period $\mathrm{B}$, the median score of total clinical evaluation was 5 (3-6) as regards staples, 5 (2-6) as regards glue, 3 (2-4) as regards intradermal B and 3 (2-4) as regards intradermal C. Staples were significantly different from glue $(\mathrm{P}=0.004)$, intradermal $\mathrm{B}$ $(\mathrm{P}<0.001)$, and intradermal $\mathrm{C}(\mathrm{P}<0.001)$.

In period $C$, the median score of total clinical evaluation was 5 (2-7) as regards staples, 5 (2-6) as regards glue, 3 (2-4) as regards intradermal B and 3 (2-4) as regards intradermal C. Staples differed significantly from intradermal $B(P=0.005)$ and intradermal $\mathrm{C}(\mathrm{P}=0.002)$.

In period $\mathrm{D}$, the median score of the total clinical evaluation was 4 (1-6) as regards staples, 3 (1-5) as regards glue, 2 (1-3) as regards intradermal B and 2 (1-3) as regards intradermal C. Staples were significantly different from intradermal B $(\mathrm{P}=0.001)$ and intradermal C $(\mathrm{P}=0.001)$. 
In period E, the median score of the total clinical evaluation was 3(1-5) as regards staples, 3 (1-5) as regards glue, 1 (0-4) as regards intradermal B and 2 (1-3) as regards intradermal C. Glue differed significantly from intradermal $\mathrm{B}(\mathrm{P}<0.001)$ and intradermal $\mathrm{C}(\mathrm{P}<0.001)$.

The median score of the total clinical evaluation for each technique in each period is shown in Figure 17.

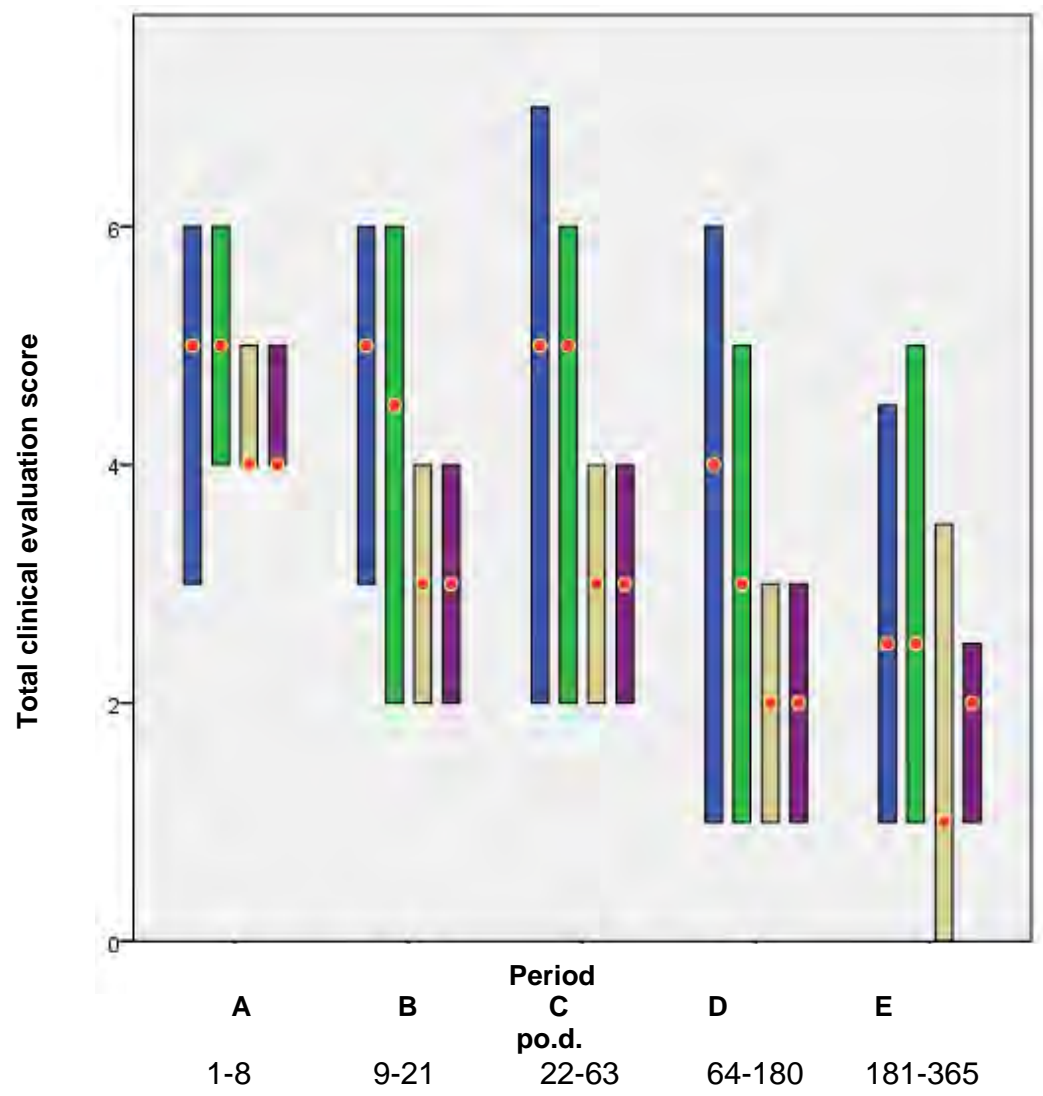

Figure 17. Median (red dot) and interquartile range (column) of total clinical evaluation score for each technique (blue: staples, green: glue, gray: intradermal B and purple: intradermal C) in each period.

\section{Ultrasonographic evaluation}

Before skin incision, in all ultrasound scans the epidermis was clearly visible as a relatively thick, mildly non-uniform, hyperechoic linear layer. The thickness of this layer varied slightly between animals and between different cutaneous sites (Figure 18). The margin between epidermis and dermis was also visible. The dermis had a granular echotexture that appeared to become more linear in the deeper parts. The superficial dermis had a granular appearance more loosely arranged with thin and irregularly distributed small echoes that probably aroused from the collagen fibers. The deep dermis contained thicker linear echoes orientated more parallel to the skin surface. Subcutaneous tissue was recognized at a greater depth, as a thicker layer characterized by an inhomogeneous hypoechoic or non-echogenic pattern (compared 
with the overlying layers), containing thin linear hyperechoic areas or bands representing connective septa (adipose nature).
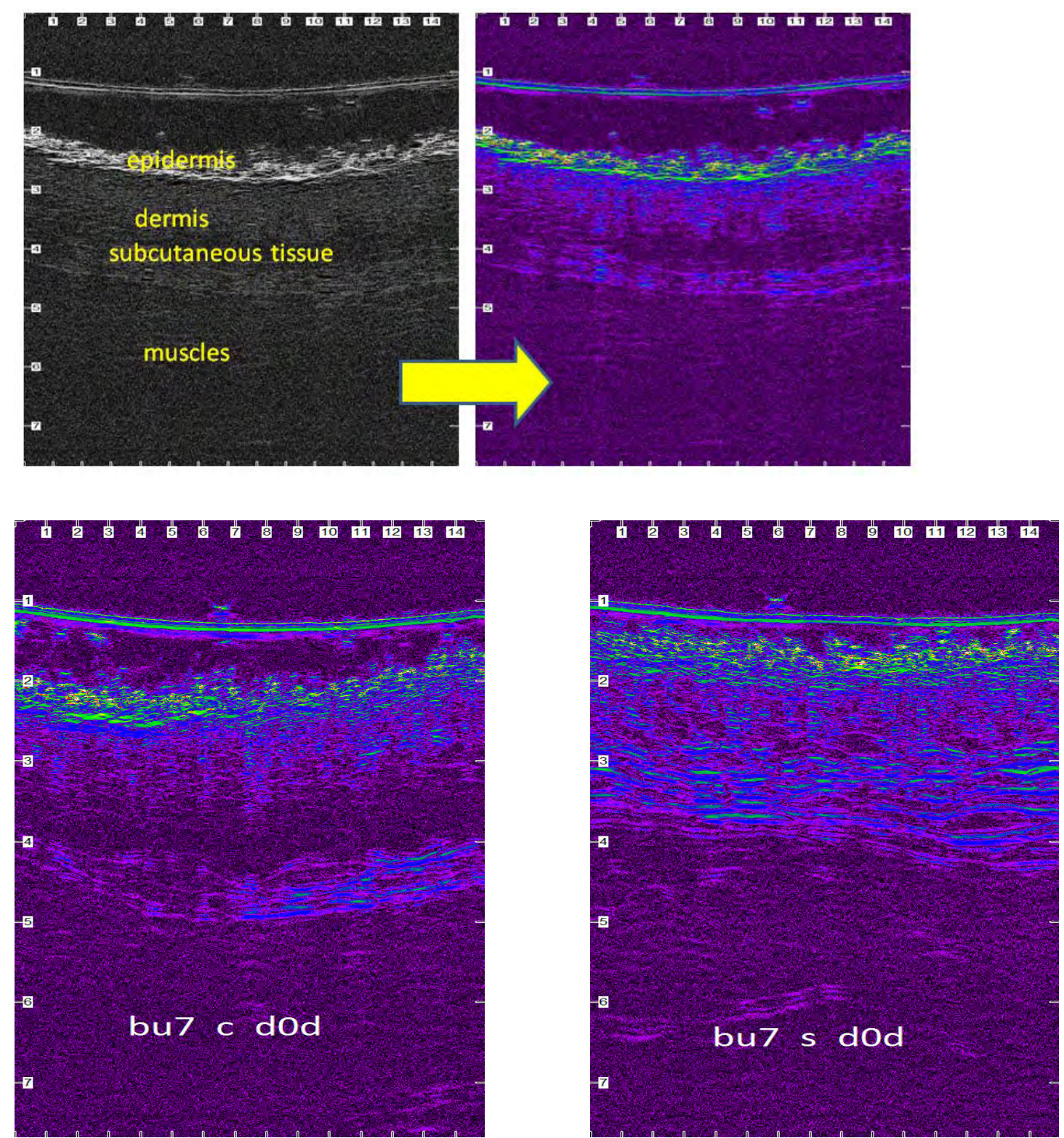

Figure 18. Ultrasonographic images of the normal canine skin of the lateral thigh of the femur. The epidermis, dermis and subcutaneous fat are clearly visible. The image is compressed laterally to facilitate viewing (c: intradermal C, s: staples).

After wound closure, the ultrasonographic image of the skin at the wound area differed from the adjacent normal one. The shape of the epidermis had been deformed, and instead of being parallel to the surface of the body, it was creating a cone that protruded 1-2 millimeters above it. The area of the dermis at the incision site was enlarged and hypoechogenic compared to the normal adjacent one, being two to three times thicker, depending on the technique and the time of examination. Figures of the scans taken on various times postoperatively are presented in Addendum 2. In 
each incision, four different tomographic planes (a, b, c, d) were performed at four sites along the scar, "a” being proximally and "d” being distal to the hip (Figure 19).

Specifically, the wound area was ultrasonographically $(\mathrm{u} / \mathrm{s})$ recorded as a hypoechoic to an anechoic small region that was defined dorsally by the two echogenic, uplifted epidermal edges, laterally by the two echogenic and thickened dermal edges and ventrally by the anechoic oedematous subcutaneous tissue. During the first postoperative days, the lower limits of the wound area were difficult to be identified due to the similar echogenicity of the subcutaneous tissue to the oedematous wound area. The lower limits of dermal edges, which were well imaged into the anechoic oedematous area, were used as guides for recording the lower wound limits. As wound healing proceeded, the wound area size and the tissue oedema diminished, and the progressive collagen deposition altered echo intensity, making wound boundaries more complex but not indistinguishable. 

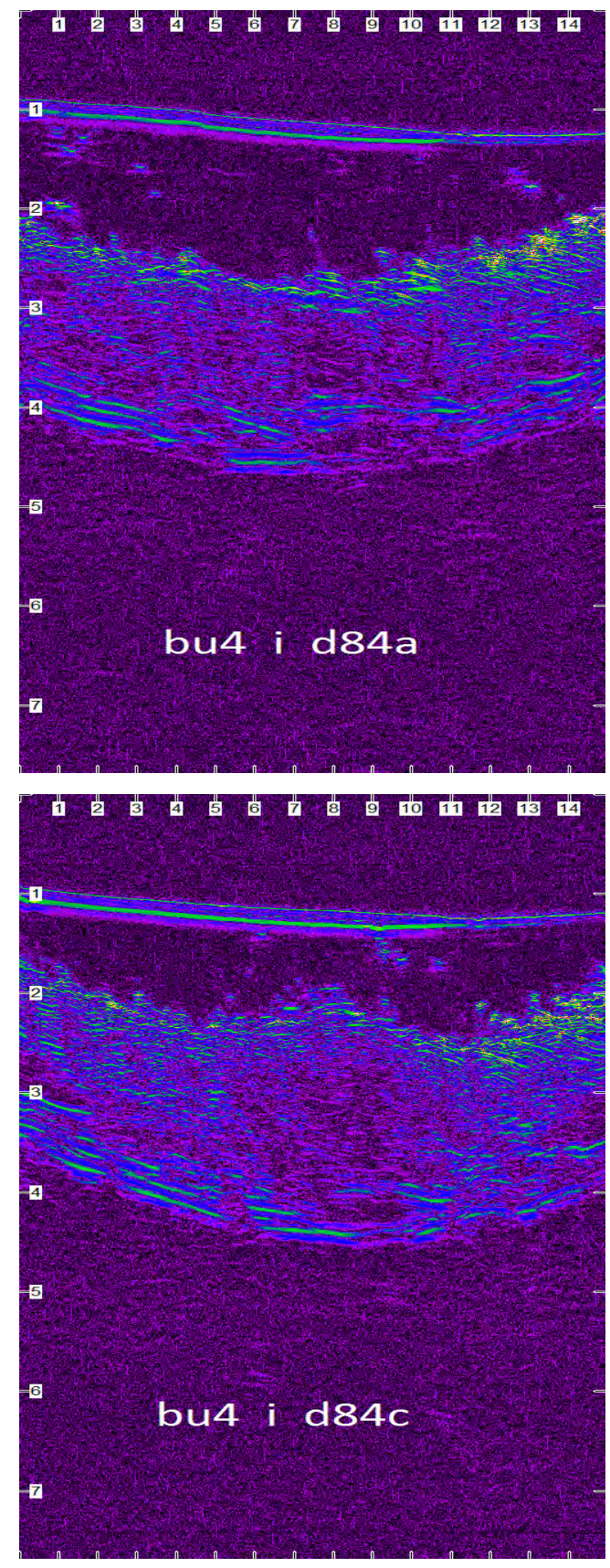

Figure 19. Ultrasonographic images of the wound area of Beagle No 4 the 84 days po., corresponding to the lateral thigh at four different sites. The wound area was of different size for each site. The image is compressed laterally to facilitate viewing (i: intradermal B).
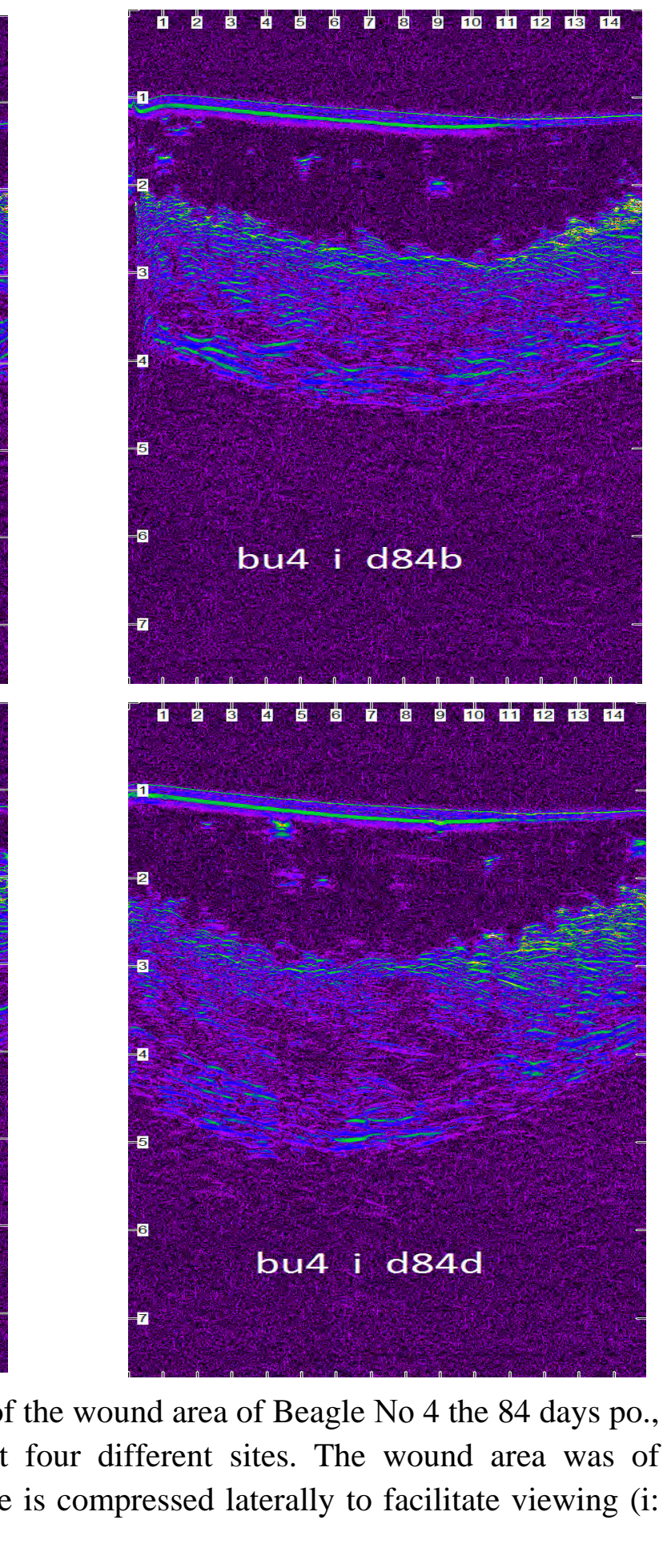

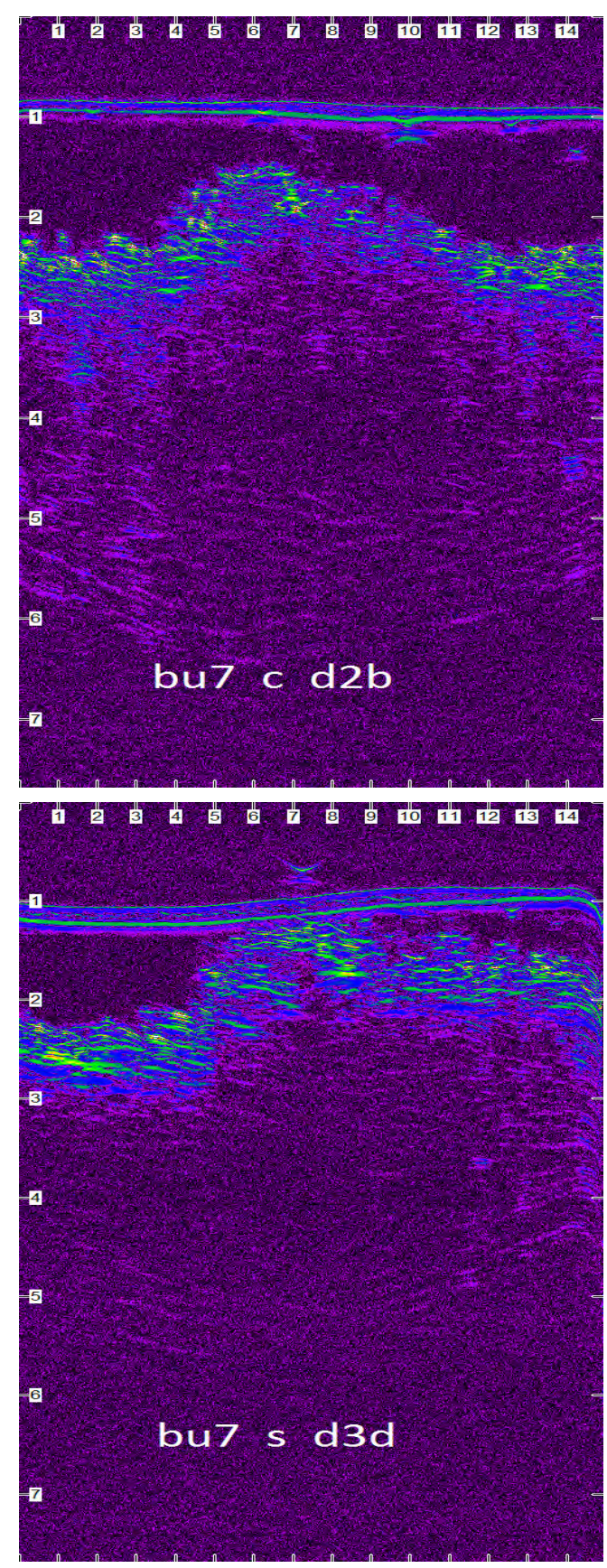

Figure 20. Ultrasonographic images of the wound area of Beagle N7 for each technique, 4 days postoperatively. The wound area was of different size for each technique. The image is compressed laterally to facilitate viewing (c: intradermal C, i: intradermal B, s: staples, g: glue).
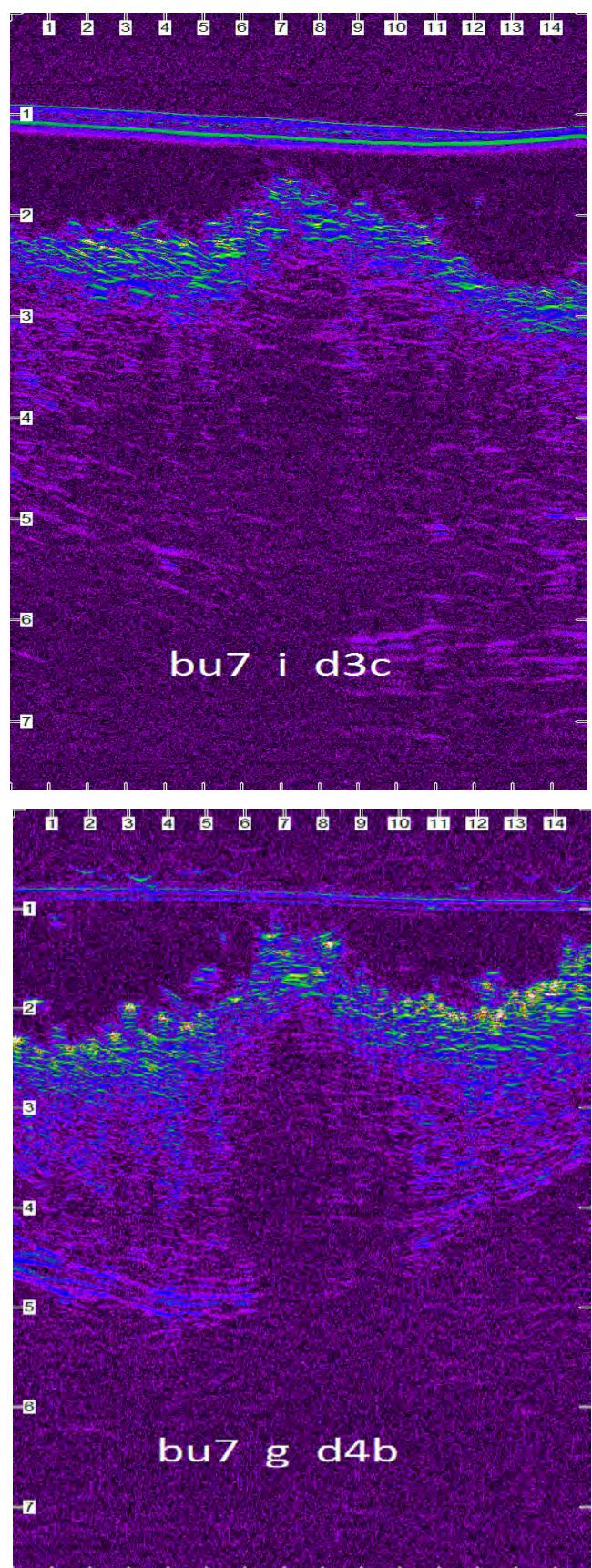

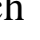



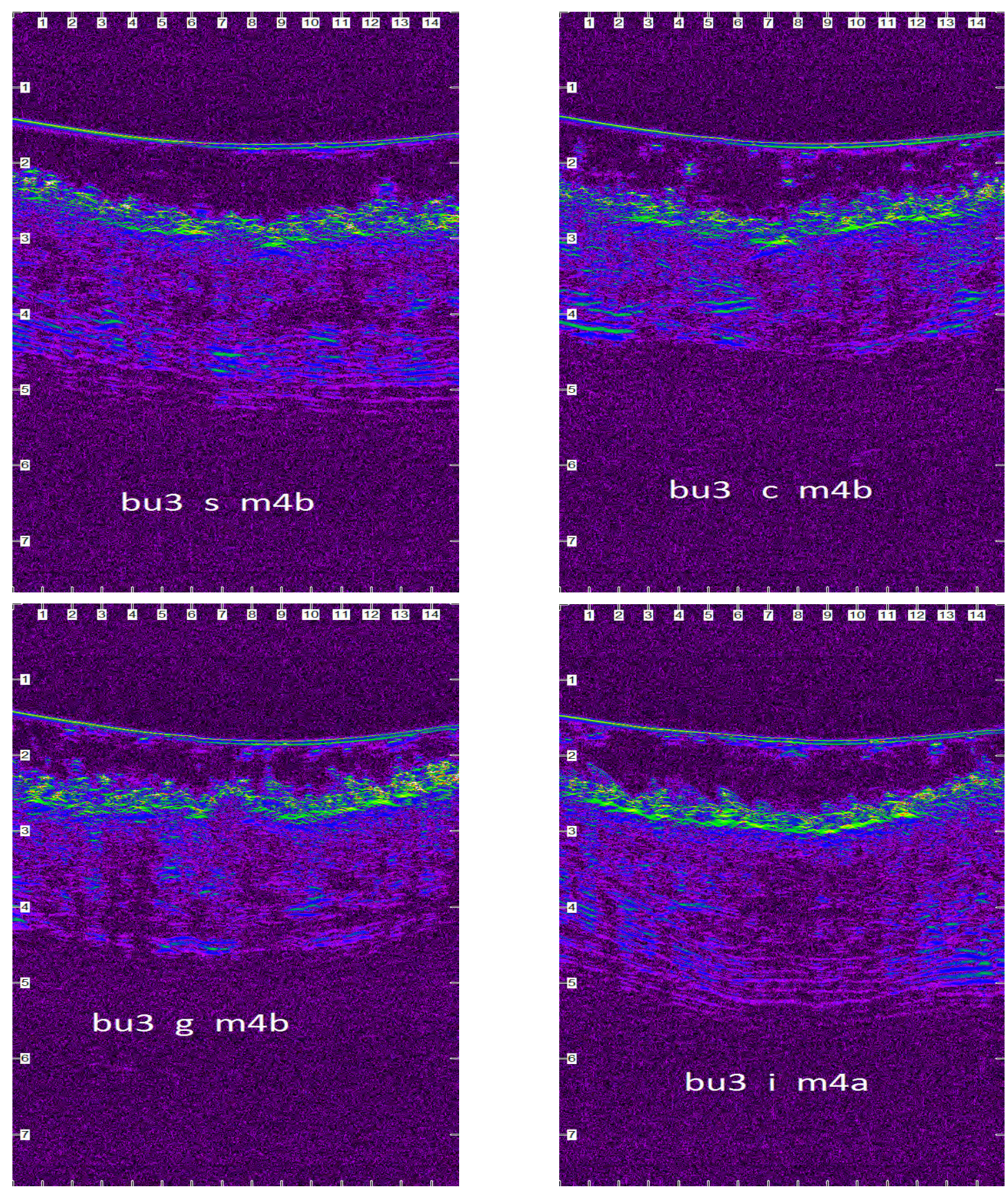

Figure 21. Ultrasonographic images of the wound area of Beagle N3 with the four different techniques, 4 months postoperatively. The wound area was of different size with each technique. The image is compressed laterally to facilitate viewing (c: intradermal C, i: intradermal B, s: staples, g: glue).

After the $15^{\text {th }}$ po.d, the ultrasonographically estimated wound area was reduced to half with all techniques. From the $40^{\text {th }}$ to the $120^{\text {th }}$ po.d., the wound area continued to reduce in size with all techniques, whilst the epidermis at wound area was losing its conical shape and was becoming flat, with a density similar to that of the adjacent normal. Wound area was depicted more clearly with glue and intradermal B compared 
to the staples and intradermal $\mathrm{C}$, as with the last two techniques the wound area was almost isoechoic to the normal adjacent skin.

After the $150^{\text {th }}$ po.d., the newly formed mature dermis at wound area became almost isoechoic to the normal adjacent skin in all incisions with all techniques.

In almost all incisions with all techniques, the epidermal edges at the incision site ultrasonographically seemed to be in close contact with each other, from the $1^{\text {st }}$ po.d. until the last day of the experiment. Although it was difficult to monitor the progress of epithelialization with the particular ultrasound scanner, that uses frequencies of $50 \mathrm{MHz}$, in some scans of the incisions closed with glue, a small epithelial gap was observed during the first postoperative day (Figure 22).

The wound area was calculated for each tomographic plan. The median area for each plane is shown in Table 4. The difference in the areas between the tomographic planes was tested with Wilcoxon Signed Ranks Test with Bonferroni correction and the results of all pairwise comparisons are shown in Table 5.

Table 4. Median area (in $\mathrm{mm}^{2}$ ), 25\% percentile, $75 \%$ percentile and interquartile length for each tomographic plane).

\begin{tabular}{|c|c|c|c|c|c|}
\hline $\begin{array}{c}\text { Tomographic } \\
\text { Plane }\end{array}$ & Valid N & $\begin{array}{c}\text { Percentile } \\
25\end{array}$ & Median & $\begin{array}{c}\text { Percentile } \\
75\end{array}$ & $\begin{array}{c}\text { Int. } \\
\text { Range }\end{array}$ \\
\hline a & 1324 & 1.92 & 3.37 & 6.67 & 4.76 \\
\hline b & 1325 & 1.67 & 3.14 & 5.89 & 4.22 \\
\hline c & 1324 & 1.78 & 3.26 & 6.32 & 4.54 \\
\hline d & 1320 & 1.99 & 3.55 & 6.51 & 4.53 \\
\hline
\end{tabular}

Table 5. Differences in wound areas between tomographic planes

\begin{tabular}{|c|c|c|c|}
\hline Tomographic plane & b & c & d \\
\hline a & $<\mathbf{0 . 0 0 1}$ & $<\mathbf{0 . 0 0 1}$ & 0.280 \\
\hline b & & $\mathbf{0 . 0 0 3}$ & $<\mathbf{0 . 0 0 1}$ \\
\hline c & & & $<\mathbf{0 . 0 0 1}$ \\
\hline
\end{tabular}

As ultrasonographically the estimated wound area differed significantly between the four different tomography planes of each incision, the mean area was calculated and used for further evaluation. The median u/s estimated wound area for each technique at each time point is presented in Figure 23. 

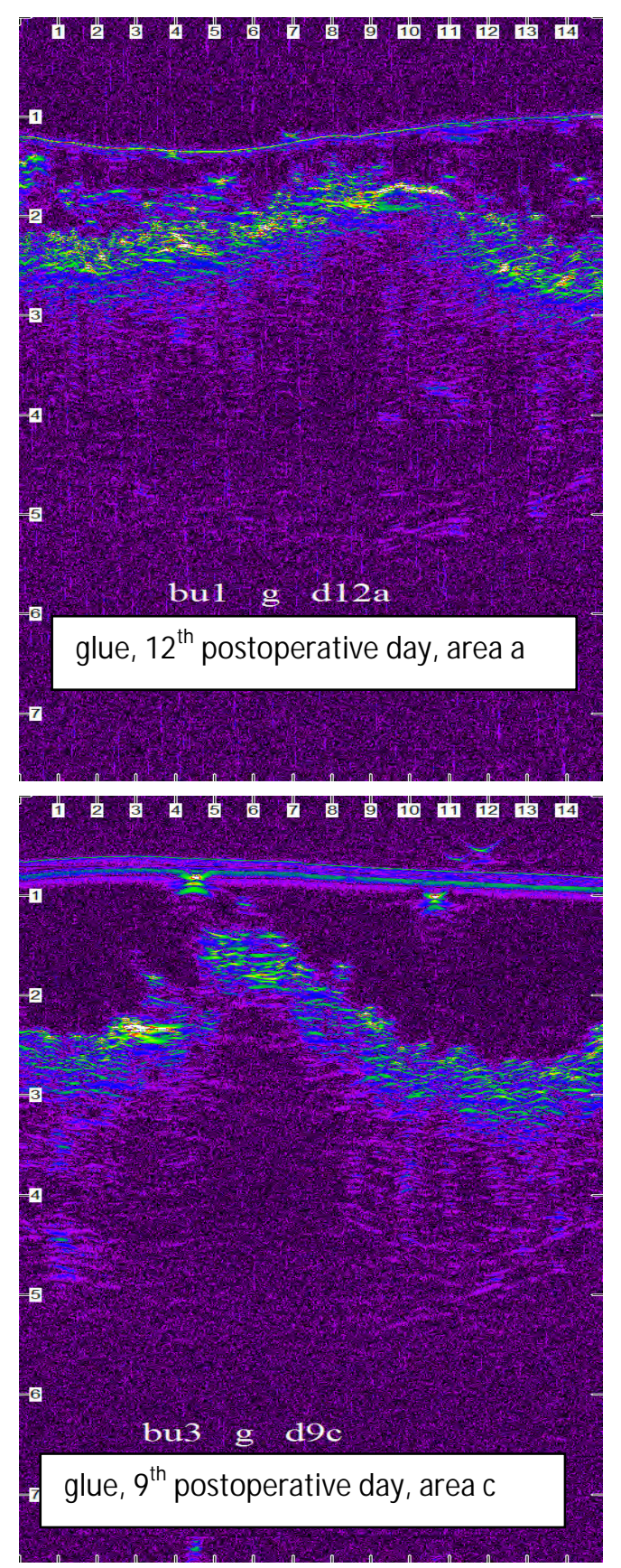

Figure 22. Ultrasonographic images of the wound area closed with glue, during the first postoperative days. The epithelial gap is evident between the epididymal edges at wound area. The image is compressed laterally to facilitate viewing (g: glue). 


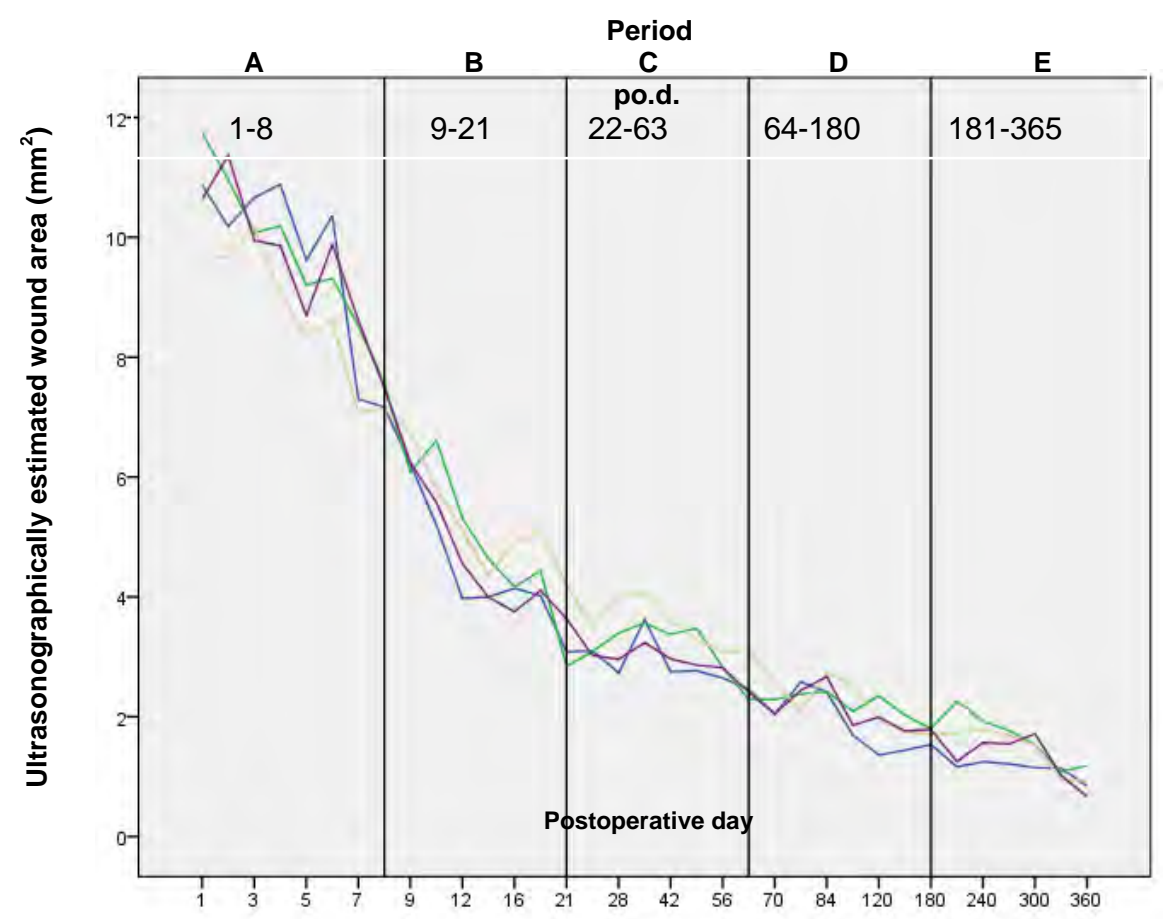

Figure 23. Median $\mathrm{u} / \mathrm{s}$ estimated wound area (in $\mathrm{mm}^{2}$ ) for each technique (blue: staples, green: glue, gray: intradermal B and purple: intradermal C) at each time point.

In period A, the median $\mathrm{u} / \mathrm{s}$ estimated wound area was $9.66(7.39-11.46)$ as regards staples, 9.74 (7.74-11.15) as regards glue, 8.60 (7.13-10.31) as regards intradermal B and 9.50 (7.87-11.20) as regards intradermal $C$, with no significant differences observed between the techniques.

In period $\mathrm{B}$, the median $\mathrm{u} / \mathrm{s}$ estimated wound area was 4.15 (3.53-5.29) as regards staples, 4.93 (3.62-6.41) as regards glue, 4.93 (4.39-5.74) as regards intradermal B and 4.54 (3.55-5.50) as regards intradermal C. Staples differed significantly from intradermal $\mathrm{B}(\mathrm{P}=0.007)$.

In period $\mathrm{C}$, the median $\mathrm{u} / \mathrm{s}$ estimated wound area was 2.73 (2.19-3.69) as regards staples, 3.15 (2.38-4.22) as regards glue, 3.23 (2.67-4.26) as regards intradermal B and 2.86 (2.20-3.65) as regards intradermal C. Staples differed significantly from intradermal $\mathrm{B}(\mathrm{P}=0.006)$.

In period $\mathrm{D}$, the median $\mathrm{u} / \mathrm{s}$ estimated wound area was $2.06(1.46-2.62)$ as regards staples, 2.26 (1.60-2.65) as regards glue, 2.15 (1.45-2.90) as regards intradermal B and 1.89 (1.42-2.57) as regards intradermal C, with no significant differences between the techniques.

In period E, the median $\mathrm{u} / \mathrm{s}$ estimated wound area was $1.12(0.75-1.86)$ as regards staples, 1.51 (1-2.34) as regards glue, 1.45 (0.7-2.21) with intradermal B and 1.34 (0.8-2.24) as regards intradermal $\mathrm{C}$, with no significant differences between the techniques. The median of the estimated wound area (in $\mathrm{mm}^{2}$ ) for each technique in each period is shown in Figure 24. 


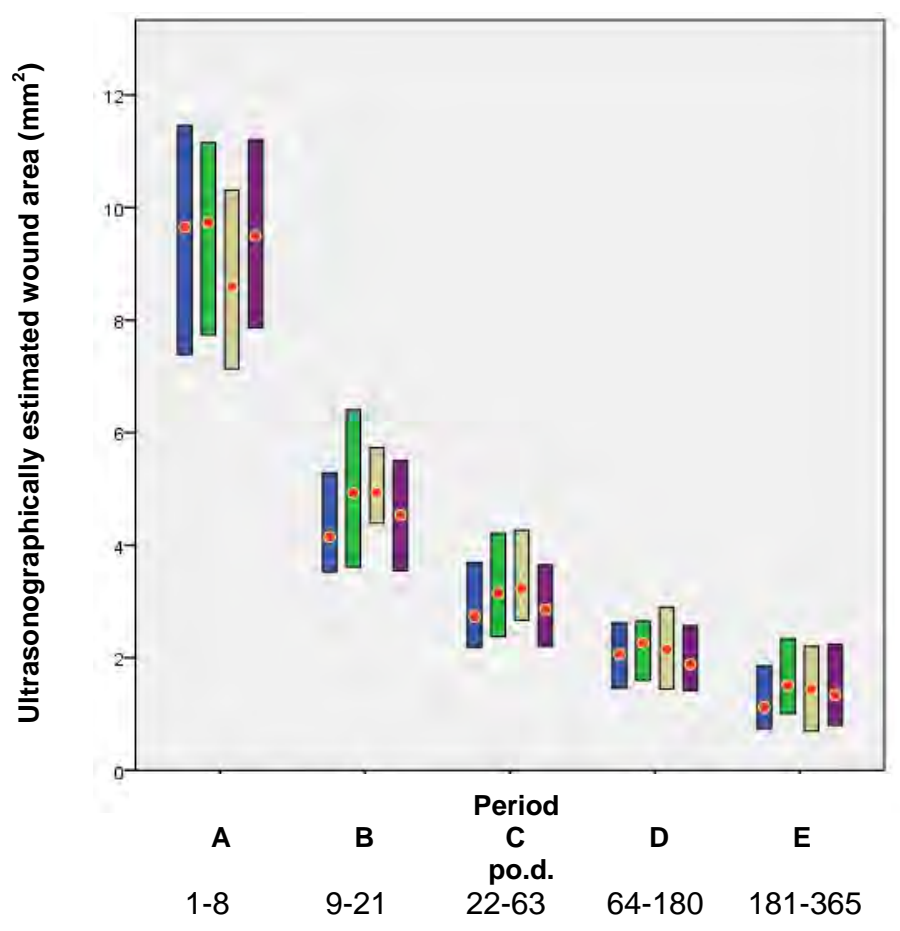

Figure 24. Median (red dot) and interquartile range (column) of $\mathrm{u} / \mathrm{s}$ estimated wound area (in $\mathrm{mm}^{2}$ ) for each technique (blue: staples, green: glue, gray: intradermal B and purple: intradermal C) in each period.

\section{Histological evaluation}

\section{Necrosis}

No necrosis was observed in any tissue examined.

\section{Oedema}

In period A, oedema was observed only in $4 / 10$ of the samples with staples and it was evaluated with score 1 . With glue and intradermal $B$ it was observed in 1/10 samples with score 2, and in 5/10 samples with score 1, respectively. With intradermal C it was observed in 5/10 samples with score 1 . In period B, oedema was presented only in 1/10 samples with staples and was evaluated with score 1 . In periods C, D and E, no oedema was observed with any technique.

\section{Inflammation}

The inflammation score for each technique is presented in Table 6.

Table 6. The incidence of each inflammation score for each technique.

\begin{tabular}{|c|c|c|c|c|c|}
\hline \multirow{2}{*}{$\begin{array}{l}\text { INFLAMMATION } \\
\text { SCORE }\end{array}$} & \multicolumn{4}{|c|}{ TECHNIQUE } & \multirow{2}{*}{ Tota } \\
\hline & Staples & Glue & intradermal B & intradermal C & \\
\hline 0 & 19 & 18 & 20 & 24 & 81 \\
\hline 1 & 13 & 12 & 9 & 8 & 42 \\
\hline 2 & 12 & 12 & 14 & 10 & 48 \\
\hline 3 & 6 & 8 & 7 & 8 & 29 \\
\hline Total & 50 & 50 & 50 & 50 & 200 \\
\hline
\end{tabular}




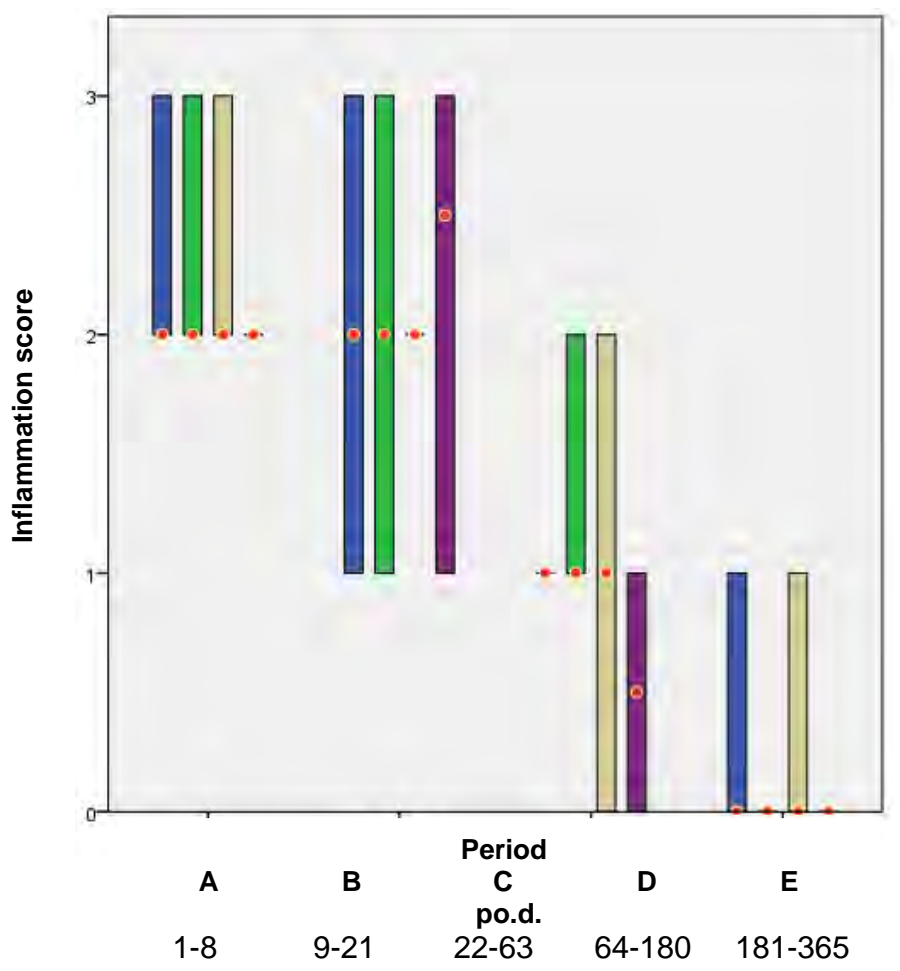

Figure 25. Median (red dot) and interquartile range (column) of inflammation score for each technique (blue: staples, green: glue, gray: intradermal B and purple: intradermal C) in each period.

In period A, the median inflammatory reaction was evaluated with score 2 (2-3) as regards staples, glue, and intradermal B and with score 2 (2-2) as regards intradermal C. In period B, the median inflammatory reaction was evaluated with score 2 (1-3) as regards staples and glue, with score $2(2-2)$ as regards intradermal B and with score 3 (1-3) as regards intradermal C. In period C, it was evaluated 1 (1-1) in staples, 1 (1-2) in glue, $1(0-2)$ in intradermal B and $1(0-1)$ in intradermal C. In period D, the median inflammation was $0(0-1)$ in staples and intradermal $B$ and $0(0-0)$ in glue and intradermal C. In period E, the median inflammation was evaluated $0(0-0)$ in all techniques. No statistically significant difference was observed between the techniques in any period. The median inflammation score for each technique in each period is shown in Figure 25.

\section{Thickness of the epidermis}

In period $\mathrm{A}$, the median thickness of the epidermis at the area of wound healing (number of times the thickness of the adjacent healthy epidermis) was 2.6 (2-3.5) as regards staples, 3.5 (3-4) as regards glue, 2.5 (2.5-3) as regards intradermal B and 2.5 (2.5 to 2.5) as regards intradermal C. Glue was significantly different from intradermal C $(\mathrm{P}=0.005)$. 
In period B the median thickness of the epidermis at the area of wound healing was 2 (2-3) as regards staples, 3 (3-4) as regards glue, 2 (2-3) as regards intradermal B and $2(2-3)$ as regards intradermal C. Glue was significantly different from intradermal B $(\mathrm{P}=0.009)$.

In period $\mathrm{C}$ the median thickness of epidermis at the area of wound healing was 1.3 (1.2-1.5) as regards staples, 2 (2- 2) as regards glue, $1.6(1.5-2)$ as regards intradermal $\mathrm{B}$ and $1.4(1.2-1.5)$ as regards intradermal $\mathrm{C}$. Glue differed significantly from the staples $(\mathrm{P}<0.001)$ and intradermal $\mathrm{C}(\mathrm{P}=0.003)$.

In period $\mathrm{D}$, the median thickness of the epidermis at the area of wound healing was 1.2 (1-1.3) as regards staples, 1.3 (1.1-1.5) as regards glue, 1.2 (1-1.3) as regards intradermal B and 1.1 (1-1.3) as regards intradermal C. No significant differences were observed between the techniques.

In period $\mathrm{E}$, the median thickness of the epidermis at the area of wound healing was 1 (1-1.1) as regards staples, 1 (1-1.2) as regards glue, 1 (1-1.1) as regards intradermal B and 1.1 (1-1.2) as regards intradermal C. No significant differences were observed between the techniques. The median thickness of the epidermis at the wound area for each technique in each period is shown in Figure 26.

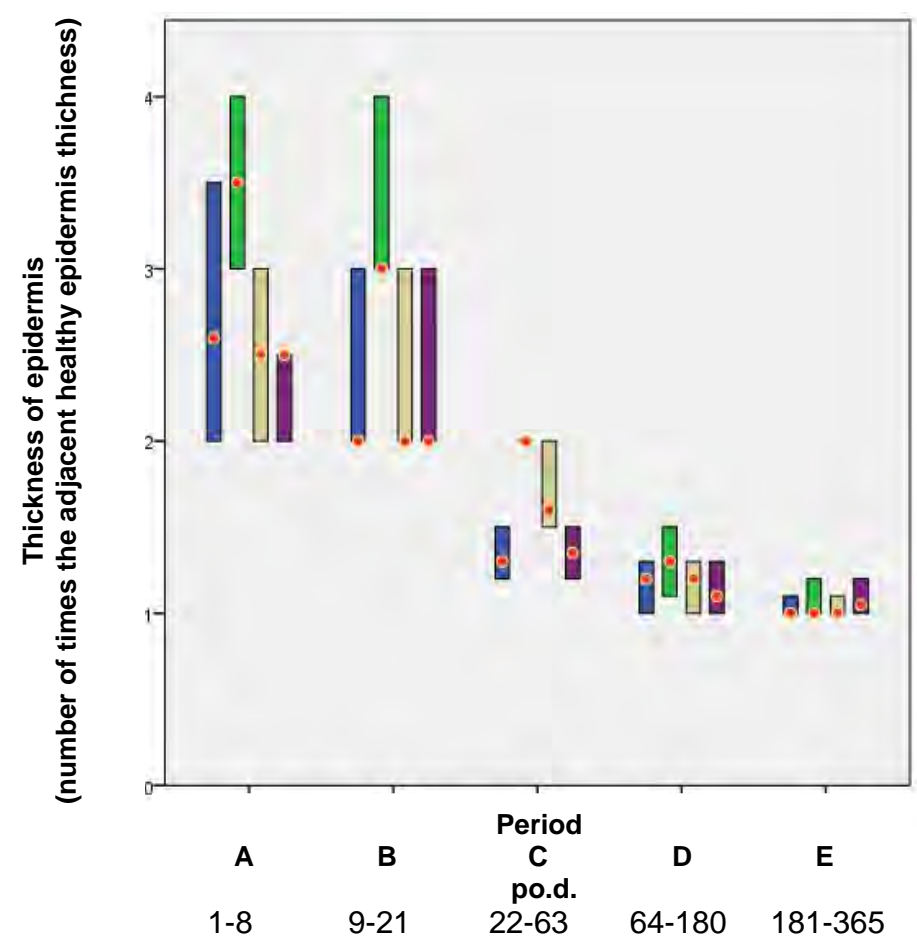

Figure 26. Median (red dot) and interquartile range (column) thickness of the epidermis at the wound area (number of times the adjacent healthy epidermis thickness) for each technique (blue: staples, green: glue, gray: intradermal B and purple: intradermal C) in each period.

\section{Epithelial gap}

Epithelial gap was observed in only three samples as regards glue on the $7^{\text {th }}$ po.d., while epithelial bridging had been complete on the $14^{\text {th }}$ po.d. for all samples. In 
Beagle No 1, the width of the gap was $0.4 \mathrm{~mm}$, in Beagle No 2 it was $0.45 \mathrm{~mm}$ and in Beagle No 3 it was $1.26 \mathrm{~mm}$.

\section{Scar width}

The histologically estimated median scar width (in $\mathrm{mm}$ ) for each technique in each period is presented in Table 7 and in Figure 27. No statistically significant difference was observed between the techniques $(\mathrm{P}>0.043)$.

Table 7. Histologically estimated median scar width (in $\mathrm{mm}$ ) for each technique in each period.

\begin{tabular}{|c|l|c|c|c|}
\hline Period & Technique & Percentile 25 & Median & Percentile 75 \\
\hline \multirow{4}{*}{ A } & staples & 0.45 & 0.54 & 1.04 \\
\cline { 2 - 5 } & glue & 0.59 & 0.9 & 0.99 \\
\cline { 2 - 5 } & intradermal B & 0.45 & 0.54 & 0.68 \\
\cline { 2 - 5 } & intradermal C & 0.54 & 0.68 & 0.68 \\
\hline \multirow{4}{*}{ B } & staples & 0.63 & 0.68 & 0.81 \\
\cline { 2 - 5 } & glue & 0.54 & 0.68 & 0.81 \\
\cline { 2 - 5 } & intradermal B & 0.45 & 0.56 & 0.68 \\
\cline { 2 - 5 } & intradermal C & 0.54 & 0.63 & 0.81 \\
\hline \multirow{4}{*}{ C } & staples & 0.5 & 0.54 & 0.9 \\
\cline { 2 - 5 } & glue & 0.5 & 0.59 & 0.9 \\
\cline { 2 - 5 } & intradermal B & 0.45 & 0.52 & 0.54 \\
\cline { 2 - 5 } & intradermal C & 0.45 & 0.52 & 0.81 \\
\hline \multirow{4}{*}{ D } & staples & 0.36 & 0.5 & 0.77 \\
\cline { 2 - 5 } & glue & 0.45 & 0.59 & 0.72 \\
\cline { 2 - 5 } & intradermal B & 0.41 & 0.45 & 0.54 \\
\cline { 2 - 5 } & intradermal C & 0.36 & 0.45 & 0.68 \\
\hline \multirow{3}{*}{ E } & staples & 0.32 & 0.38 & 0.45 \\
\cline { 2 - 5 } & glue & 0.45 & 0.59 & 0.81 \\
\cline { 2 - 5 } & intradermal B & 0.41 & 0.45 & 0.45 \\
\cline { 2 - 5 } & intradermal C & 0.36 & 0.45 & 0.45 \\
\hline
\end{tabular}




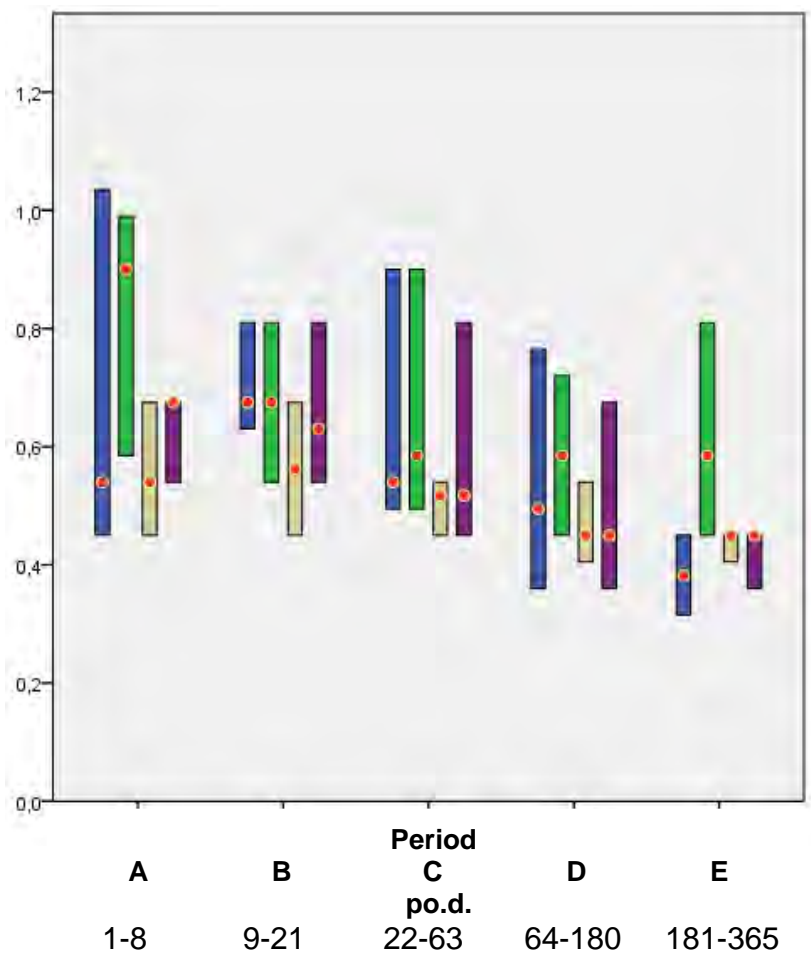

Figure 27. Histologically estimated median (red dot) and interquartile range (column) of the scar width (in $\mathrm{mm}$ ) for each technique (blue: staples, green: glue, gray: intradermal B and purple: intradermal C) in each period.

\section{Presence of suture material}

The presence of suture material or its initial position was identified at 30 samples as regards intradermal B and intradermal C; poliglecaprone 25 was found in 24 of these samples, whilst polypropylene was found in the other 6 samples. From the $14^{\text {th }}$ po.d. onwards, polypropylene was not found, as the suture material had been removed on the $10^{\text {th }}$ po.d., while the polypropylene's initial position was identified in 2 of the samples. Poliglecaprone was not found on the $180^{\text {th }}$ po.d., due to its absorption within 119 days post-implantation.

\section{Tissue reaction around the suture material}

On the $7^{\text {th }}$ po.d. the tissue reaction around the suture material consisted of macrophages and fibroblasts and it was evaluated as minimal as regards intradermal $\mathrm{C}$ where polypropylene was used. As regards intradermal $\mathrm{B}$, on the $7^{\text {th }}, 14^{\text {th, }}$ and $28^{\text {th }}$ po.d., the tissue reaction around suture material was minimal to moderate and it consisted of macrophages and fibroblasts. In some cases, the suture material was only surrounded by normal skin collagen fibers and not by cells; in other cases, it was surrounded by a small number of macrophages which were arranged in one or two layers, or it was surrounded by more than one layer of inflammatory cells and these layers were surrounded by fibroblasts and by circular collagen fibers.

From the $180^{\text {th }}$ po.d. onwards, the area, which the suture material had passed through, was not able to be detected at any tissue sample. 
Tissue reaction around the suture material as regards intradermal $\mathrm{B}$ was evaluated in periods $\mathrm{A}, \mathrm{B}$, and $\mathrm{C}$ (the suture was absorbed 90-110 days after implantation) and as regards intradermal $\mathrm{C}$ in period $\mathrm{A}$ (the suture was removed 10 days after implantation).

In period $A$, the score of tissue reaction around the suture material as regards intradermal B was 1 in 4 samples, 2 in 4 samples, and 3 in 1 sample. In period B, the score of tissue reaction as regards intradermal B was 1 in 3 samples, 2 in 4 samples, and 3 in 1 sample; in period C, was 1 in 3 samples, 2 in 1 sample and 3 in 2 samples. As regards intradermal $\mathrm{C}$, in period A the score of tissue reaction around the suture material was 1 in 4 samples. Due to the small number of samples, no statistical analysis was performed.

\section{Fibroblasts presence}

The fibroblasts presence score for each technique is presented in Table 8.

Table 8. Incidence of each fibroblast presence score for each technique.

\begin{tabular}{|c|c|c|c|c|c|c|}
\hline & \multicolumn{4}{|c|}{ TECHNIQUE } & \multirow{2}{*}{ Tota } \\
\hline & & Staples & Glue & intradermal B & intradermal C & \\
\hline \multirow{2}{*}{ FIBROBLASTS } & 2 & 24 & 25 & 23 & 28 & 100 \\
\hline & 3 & 26 & 25 & 27 & 22 & 100 \\
\hline \multicolumn{2}{|l|}{ Total } & 50 & 50 & 50 & 50 & 200 \\
\hline
\end{tabular}

In periods A and B the median fibroblasts presence score was 3 (3-3) for all techniques. In period C it was 3 (2-3) as regards staples, glue, and intradermal B, and $2(2-3)$ as regards intradermal C. In periods D and E the median fibroblasts presence score was 2 (2-2) for all techniques. No statistically significant difference was observed between the techniques.

\section{Collagen deposition}

The collagen deposition score for each technique is presented in Table 9.

Table 9. Incidence of collagen deposition score for each technique

\begin{tabular}{|c|c|c|c|c|c|}
\hline \multirow{2}{*}{$\begin{array}{c}\text { COLLAGEN } \\
\text { SCORE }\end{array}$} & \multicolumn{4}{|c|}{ TECHNIQUE } & \multirow{2}{*}{ Total } \\
\hline & staples & glue & intradermal B & intradermal C & \\
\hline 1 & 9 & 10 & 8 & 13 & 40 \\
\hline 2 & 13 & 12 & 15 & 8 & 48 \\
\hline 3 & 28 & 28 & 27 & 29 & 112 \\
\hline Total & 50 & 50 & 50 & 50 & 200 \\
\hline
\end{tabular}


In period A, the median collagen deposition score was 1 (1-1) for all techniques. In period B it was 2 (2-2) as regards staples, glue, and intradermal B, and 2 (1-2) as regards intradermal $C$. In periods $\mathrm{C}, \mathrm{D}$ and $\mathrm{E}$ the median collagen deposition score was 3 (3-3) for all techniques. No statistically significant difference was observed between the techniques in any period. The median collagen deposition score for each technique in each period is shown in Figure 28.

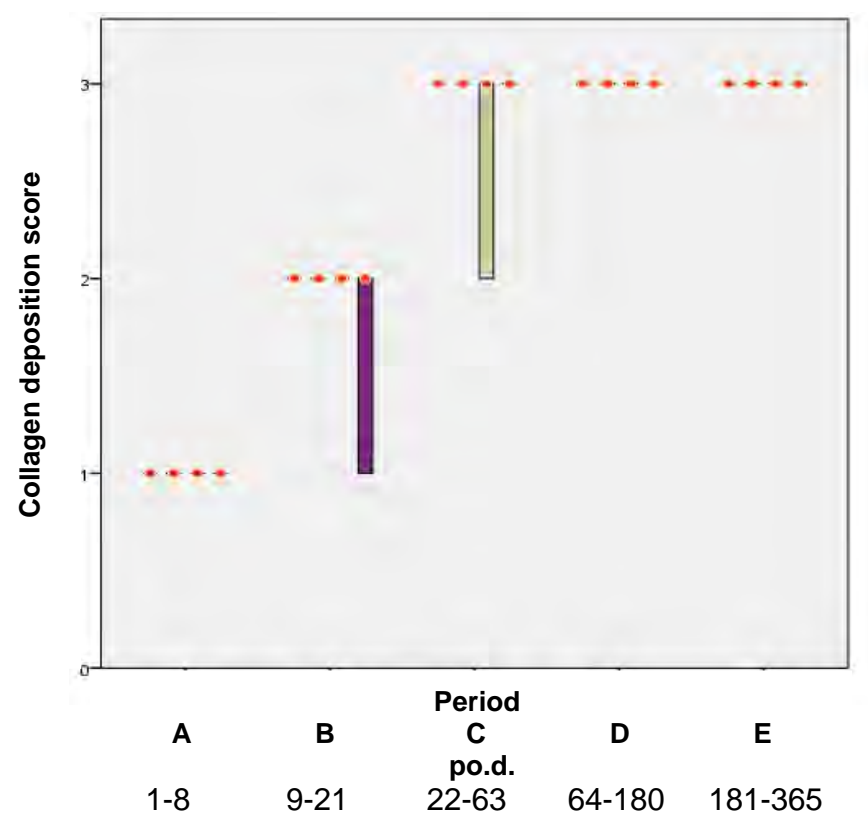

Figure 28. Median (red dot) and interquartile range (column) of collagen deposition score for each technique (blue: staples, green: glue, gray: intradermal B and purple: intradermal C) in each period.

\section{Angiogenesis}

The angiogenesis score for each technique is presented in Table 10. No statistically significant difference was observed between the techniques.

\begin{tabular}{|c|c|c|c|c|}
\hline \multirow{2}{*}{ Technique } & \multirow{2}{*}{ score } & \multicolumn{3}{|c|}{ Periods } \\
\cline { 2 - 5 } & & A & B & C \\
\hline \multirow{2}{*}{ staples } & 1 & 4 & 8 & 10 \\
\cline { 2 - 5 } & 2 & 6 & 2 & 0 \\
\hline \multirow{2}{*}{ glue } & 1 & 3 & 6 & 9 \\
\cline { 2 - 5 } & 2 & 7 & 4 & 1 \\
\hline \multirow{2}{*}{$\begin{array}{c}\text { intradermal } \\
\text { B }\end{array}$} & 1 & 4 & 6 & 8 \\
\cline { 2 - 5 } & 2 & 6 & 3 & 2 \\
\hline \multirow{2}{*}{$\begin{array}{c}\text { intradermal } \\
\text { C }\end{array}$} & 1 & 2 & 7 & 10 \\
\cline { 2 - 5 } & 2 & 8 & 3 & 0 \\
\hline
\end{tabular}

Table 10. Incidence of angiogenesis score for each technique. 


\section{Total histological evaluation}

In period $A$, the median score of the total histological evaluation was 7 (5-8) as regards staples, 8 (7-10) as regards glue, 7 (6-8) as regards intradermal B and 7 (7-8) as regards intradermal $\mathrm{C}$. In period B, it was 6 (5-7) as regards staples, 7 (6-8) as regards glue, 5 (5-6) as regards intradermal B and 7 (5-7) as regards intradermal C. In period C, it was 4 (2-5) as regards staples, 5 (4-6) as regards glue, 3(2-5) as regards intradermal B and $3(2-4)$ as regards intradermal C. In period D, it was $1(0-3)$ as regards staples, $3(1-4)$ as regards glue, $1(0-2)$ as regards intradermal B and $1(0-2)$ as regards intradermal C. Finally, in period E, it was $0(0-0)$ as regards staples, $2(0-3)$ as regards glue, $0(0-0)$ as regards intradermal $\mathrm{B}$, and $0(0-3)$ as regards intradermal $\mathrm{C}$. In all periods no significant differences were odserved between the techniques. The median total histological score for each technique in each period is shown in Figure 29.

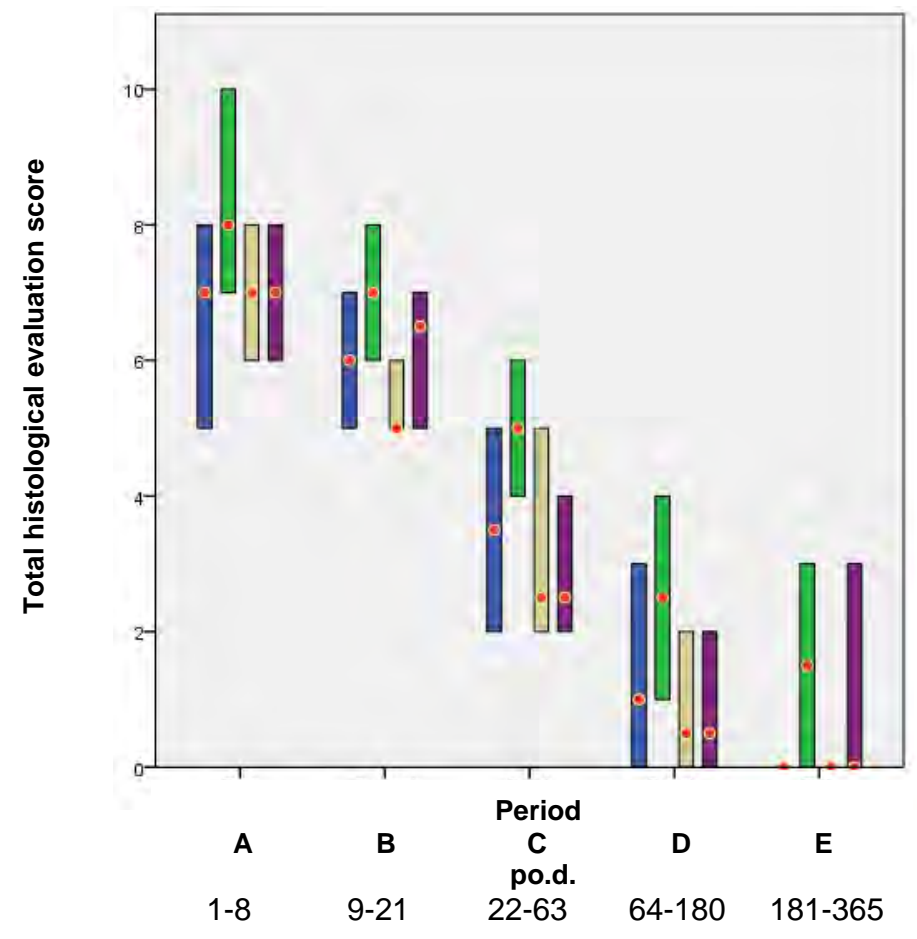

Figure 29. Median (red dot) and interquartile range (column) of score of total histological evaluation for each technique (blue: staples, green: glue, gray: intradermal B and purple: intradermal C) in each period. 


\section{Histological features}

\section{Skin stapling}

On the $7^{\text {th }}$ po.d., the main finding was the infiltration of the wound area at the site of the incision by a small number of neutrophils, macrophages, and fibroblasts (Figure $30 \mathrm{a}, \mathrm{b})$. The thickness of the epidermis was increased $\mathrm{x}$ 1.8-5 times compared to the normal epidermis (Figure 30b). The upper dermis next to the wound area was occasionally infiltrated by small numbers of macrophages and few mast cells. In two animals (B2, B10)a nodular accumulation of macrophages and lymphocytes at the subcutaneous tissue was observed (Figure 31). Foci with traumatic furunculosis were also found (B1, B8, Figure 32a,b).
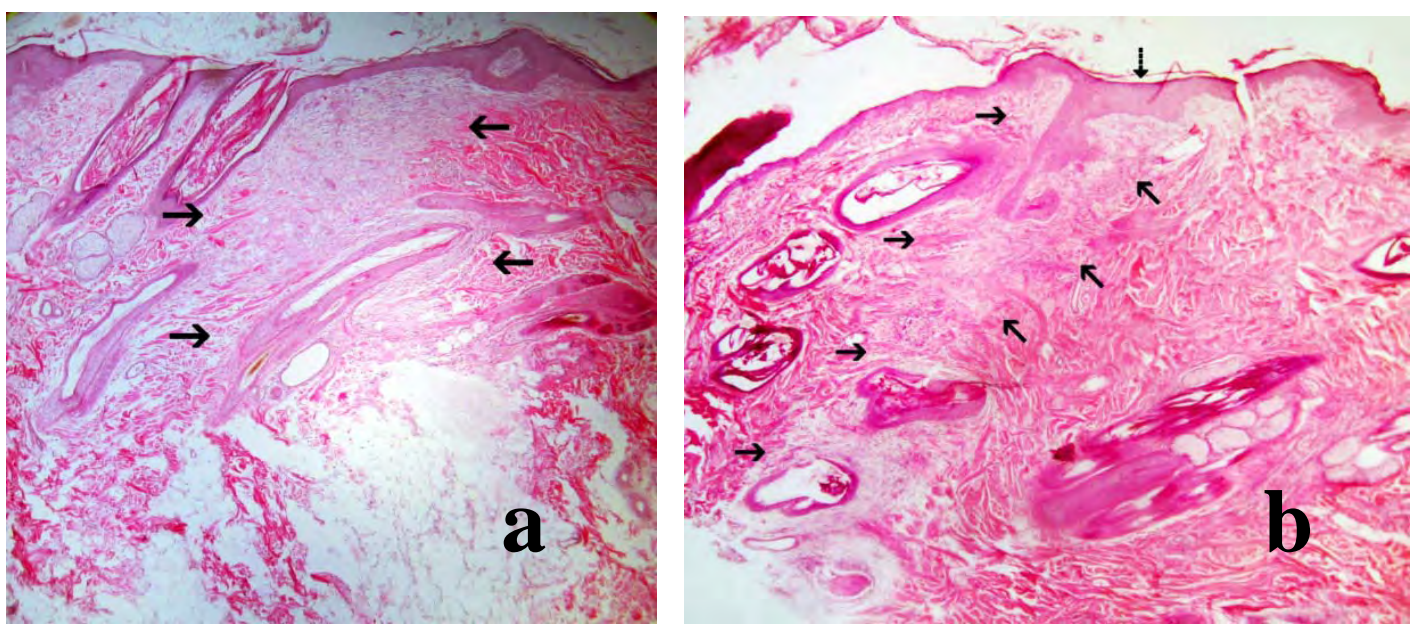

Figure 30.Wound area (between arrows) at the site of the incision on the $7^{\text {th }}$ po.d. a. Wide wound area with mild infiltration by macrophages. b. Narrow wound area with thickened epidermis ( $\mathrm{x} 5$ times the normal epidermis) covering it.

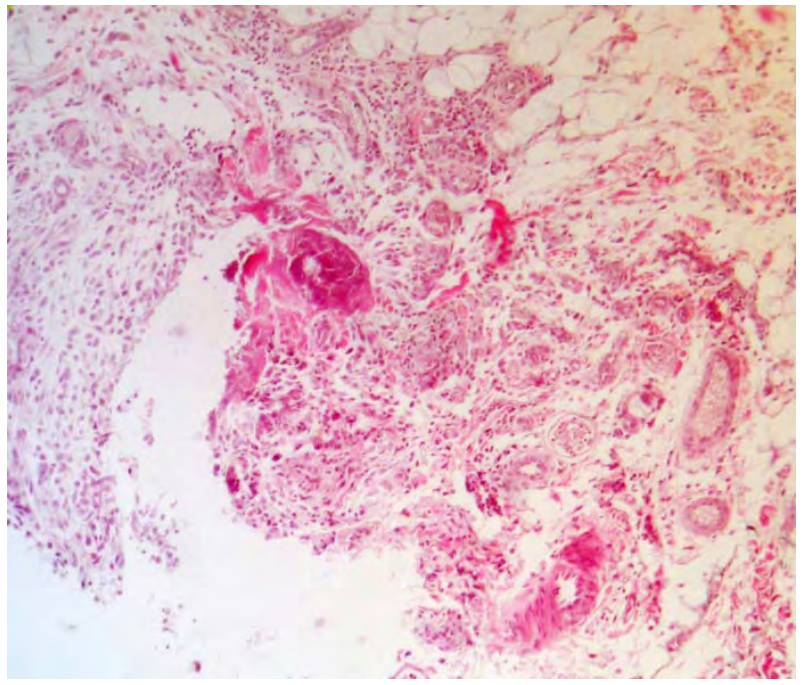

Figure 31. Wound area at the site of the incision on the $7^{\text {th }}$ po.d. Nodular accumulation of macro-phages and lymphocytes at the subcutaneous tissue. 

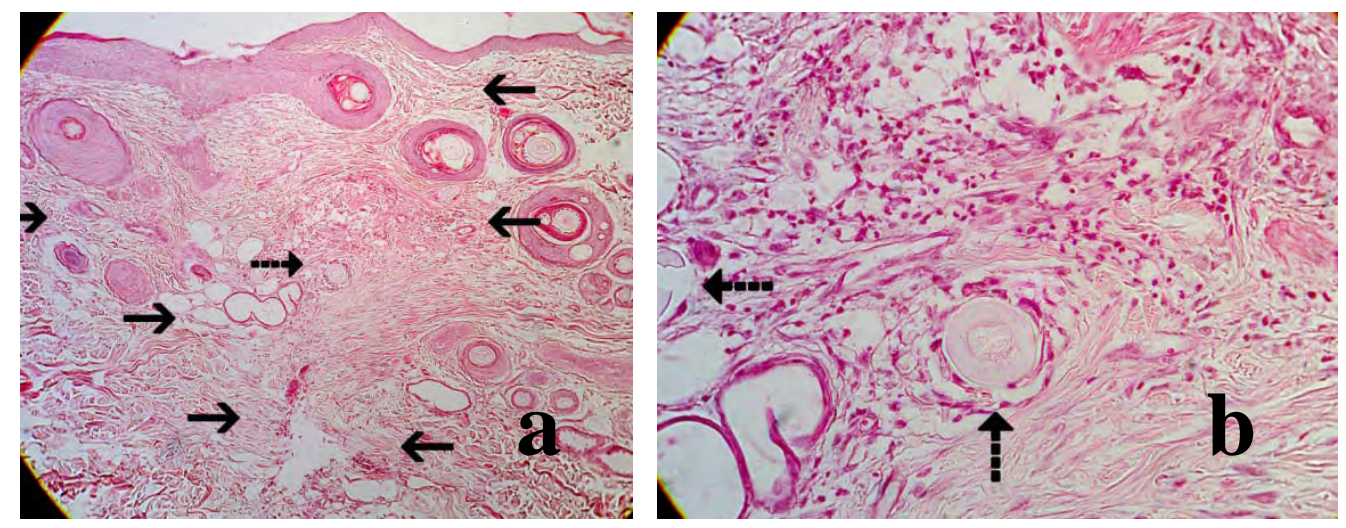

Figure 32a,b. Wound area (between solid arrows) with foci with traumatic furunculosis (hair fragments, dotted arrows) at the site of the incision, on the $7^{\text {th }}$ po.d.

On the $14^{\text {th }}$ po.d., the main finding was the infiltration of the wound area at the site of the incision, by fibroblasts and a small number of macrophages (Figure 33a, b). The thickness of the epidermis was increased x1.5-6 times compared to the normal one (Figure 33b). The upper dermis next to the wound area was occasionally infiltrated by few macrophages and mast cells. In one animal (B3), a nodular accumulation of macrophages, neutrophils, and lymphocytes was observed (Figure 34a,b). Three foci with traumatic furunculosis were also found (B2, B10) (Figure 35a,b).
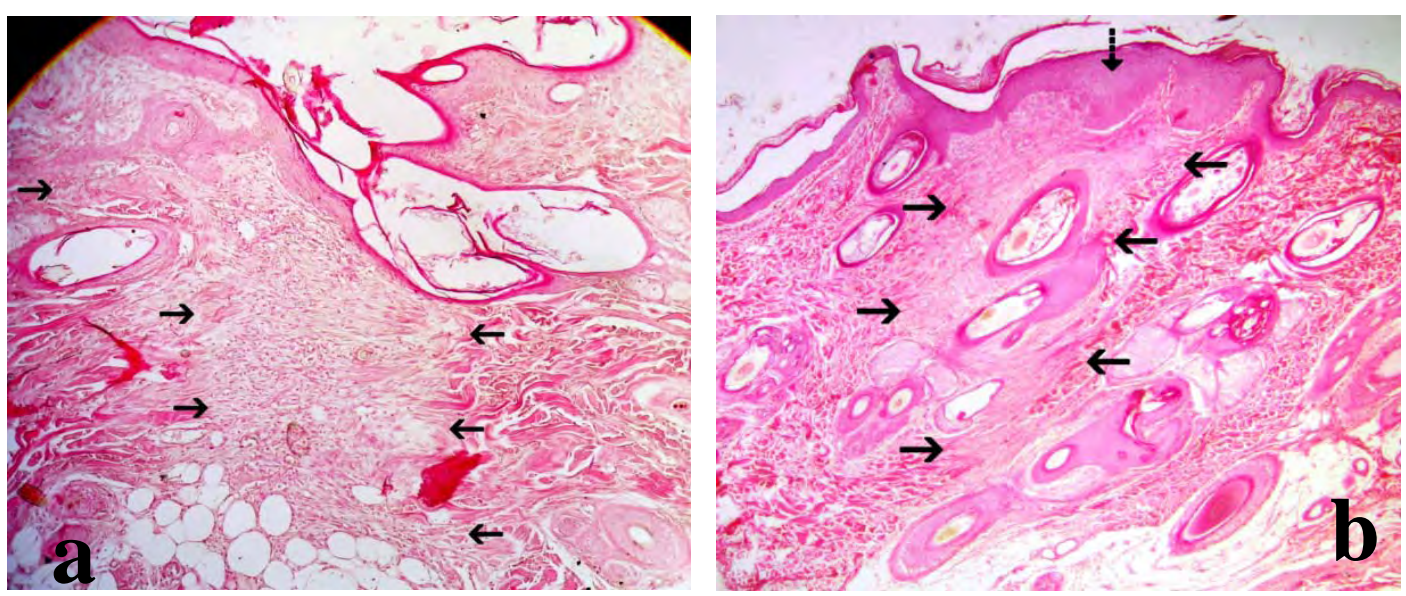

Figure 33. Wound area (between arrows) at the site of the incision on the $14^{\text {th }}$ po.d. a. Wide wound area with mild infiltration of macrophages. b. Wound area with thickened epidermis (x6 times the normal one) covering it. 

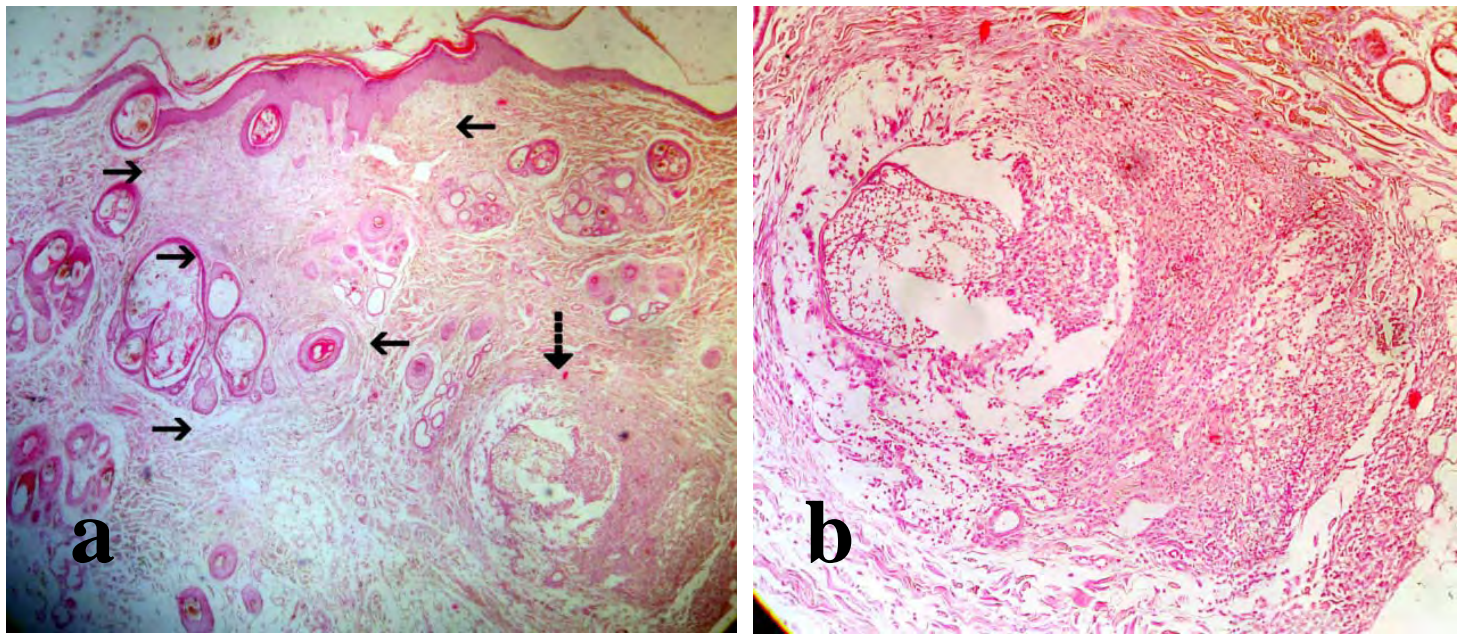

Figure 34a,b. Wound area (solid arrows) on the $14^{\text {th }}$ po.d. with epidermis thickening and a nodular accumulation of macrophages, neutrophils, and lymphocytes (dotted arrow, b).
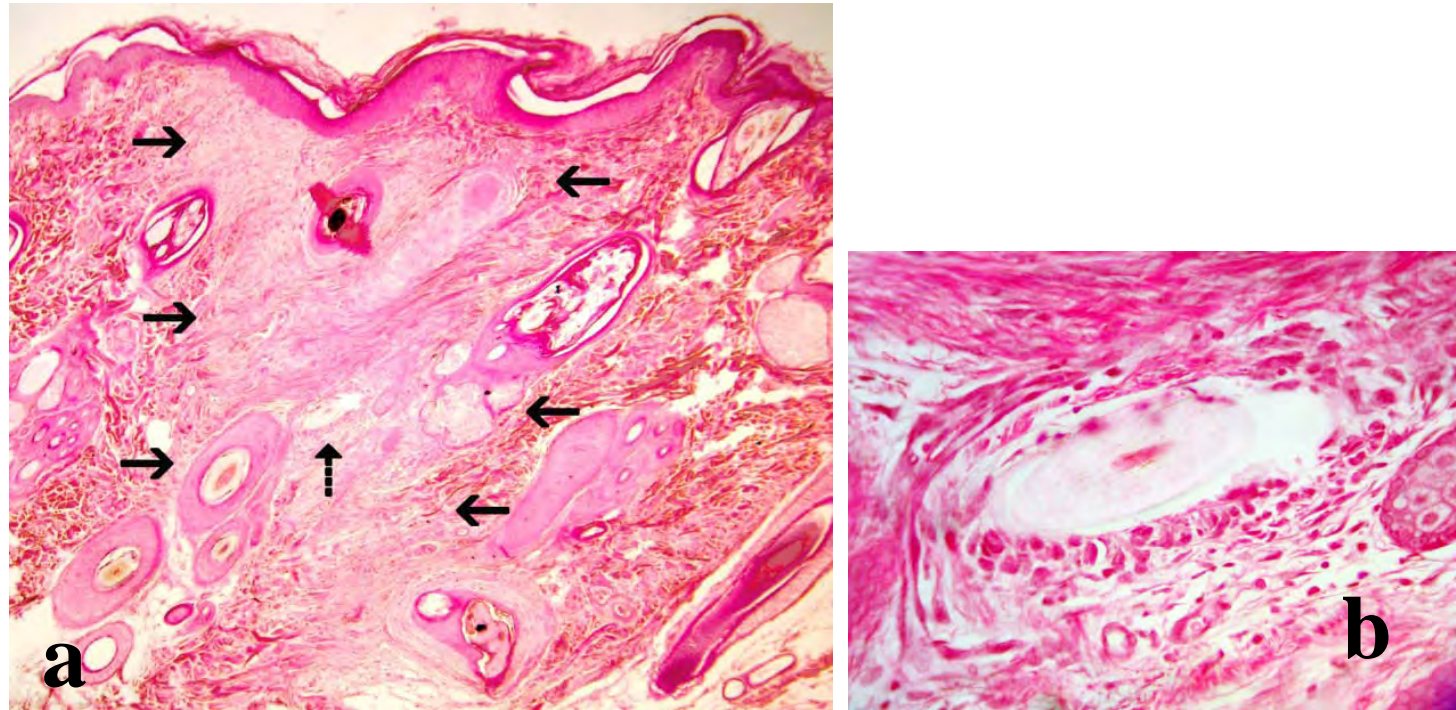

Figure 35a,b. Wound area (solid arrows) on the $14^{\text {th }}$ po.d. with mild epidermis thickening and a nodular accumulation of macrophages, neutrophils, and lymphocytes (dotted arrow, b) due to traumatic furunculosis.

On the $\mathbf{2 8}^{\text {th }}$ po.d. , the main finding was the absence of glands and hair follicles from the wound area at the site of the incision, where thinner than normal bands of collagen and a minimal number of macrophages were observed (Figure 36). The thickness of the epidermis had decreased to x1-1.5 times compared to the normal adjacent epidermis, whilst in one case it was $\mathrm{x} 2$ times the normal epidermis. Three foci with traumatic furunculosis were found (B2, B3) (Figure 37a,b). 


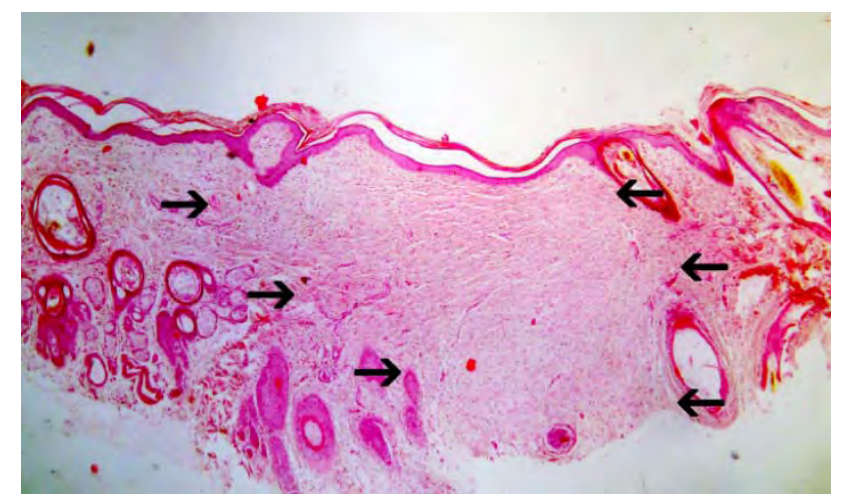

Figure 36. Very wide wound area at the site of the incision (solid arrows) on the $28^{\text {th }}$ po.d, without any glands or hair follicles, and with thinner than normal bands of collagen.
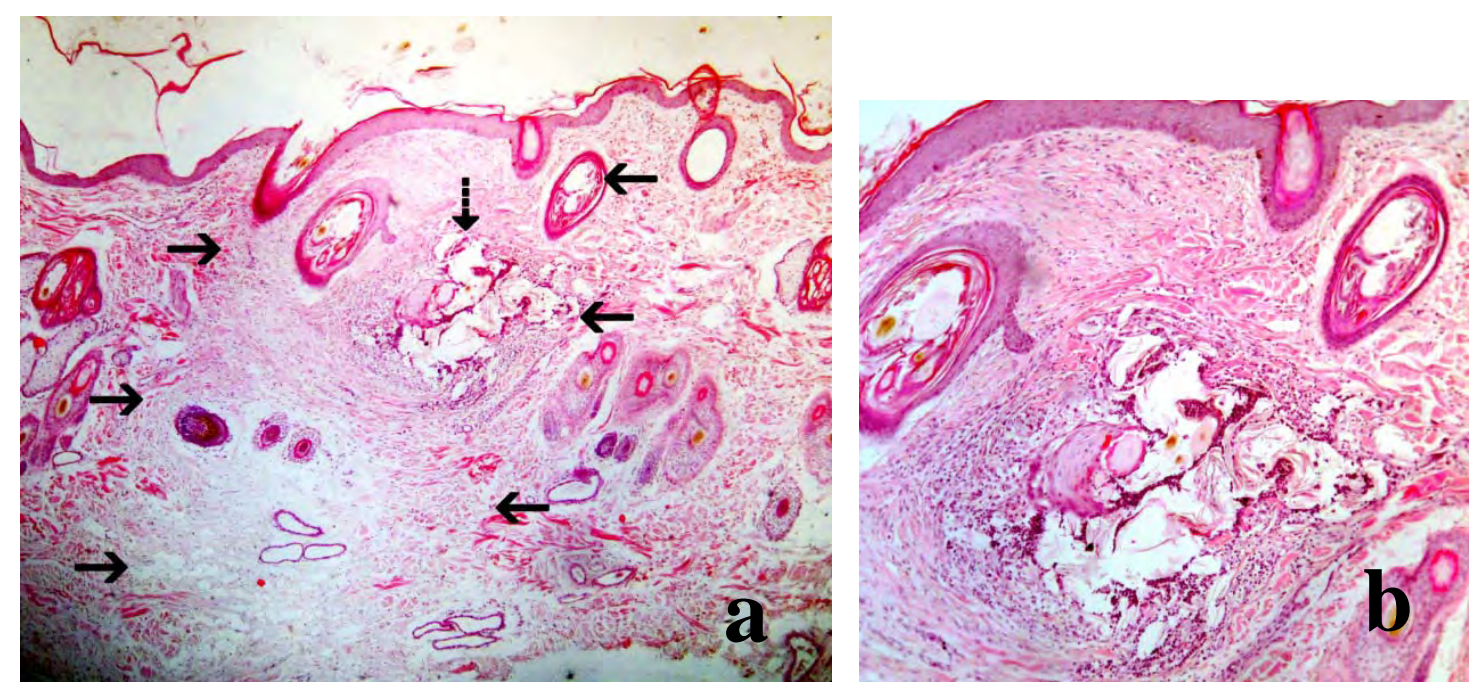

Figure 37a,b. Wound area (solid arrows) on the $28^{\text {th }}$ po.d. with mild epidermis thickening and a nodular accumulation of macrophages, neutrophils, and lymphocytes (dotted arrow, b) due to traumatic furunculosis.

On the $\mathbf{1 8 0}^{\text {th }}$ po.d., the main finding was the absence of glands and hair follicles from the wound area at the site of the incision, where thinner than normal bands of collagen were observed (Figure 38a,b). In three animals, the dermis at the wound area was infiltrated by a few macrophages, mast cells, and lymphocytes (Figure 39a,b). The thickness of the epidermis was x1-1.5 times compared to the normal adjacent epidermis. 

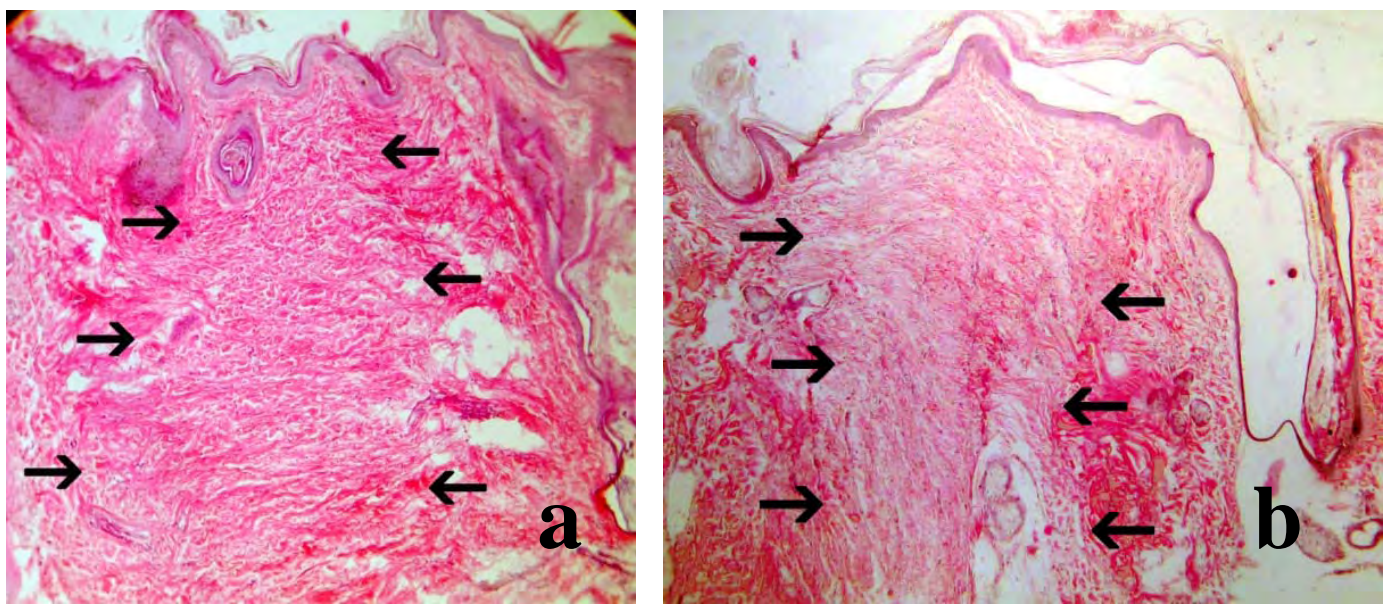

Figure 38a,b. Wide wound area at the site of the incision (solid arrows) on the $180^{\text {th }}$ po.d, without any glands or hair follicles, and with thinner than normal bands of collagen.
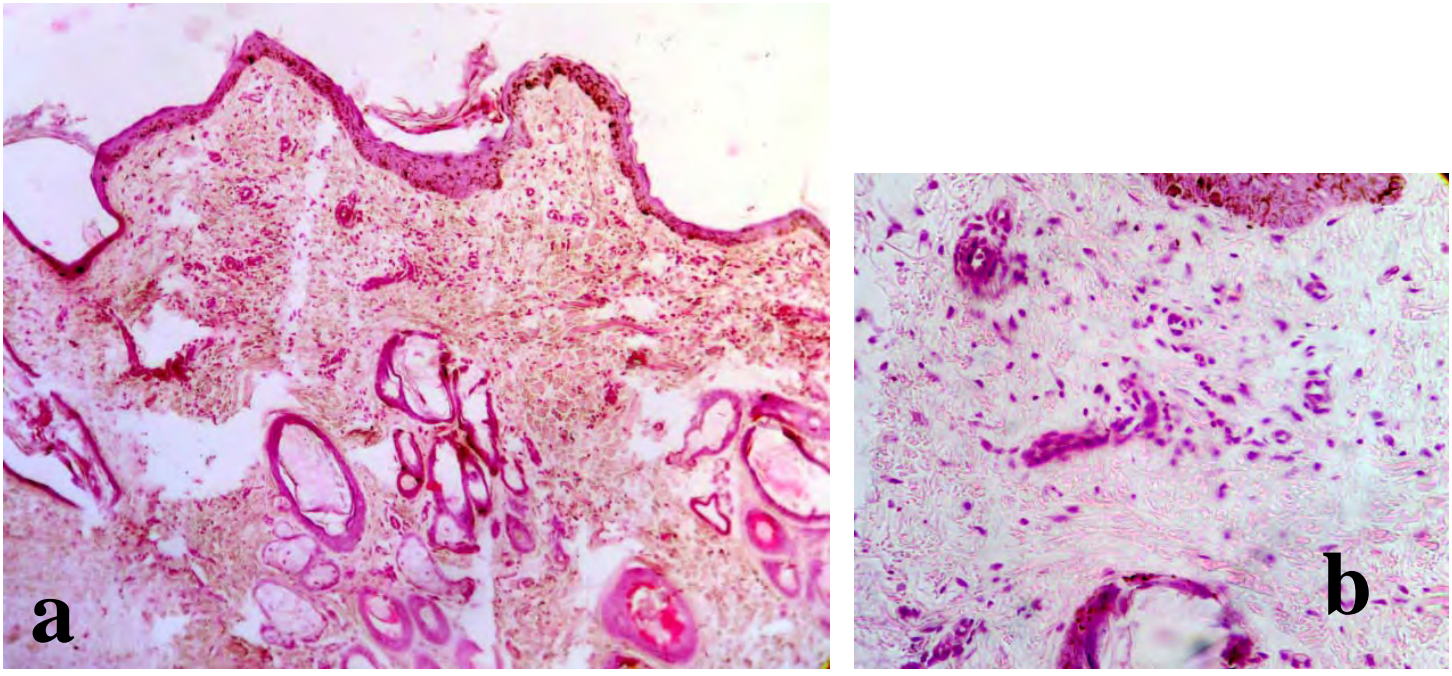

Figure 39a,b. Dermis at the wound area infiltrated by few macrophages, mast cells, and lymphocytes.

On the $365^{\text {th }}$ po.d. , the main finding was the absence of glands and hair follicles from the wound area at the site of the incision where thinner than normal collagen fiber bands were observed. The thickness of the epidermis was x1-1.2 times compared to the normal adjacent epidermis (Figure 40). 


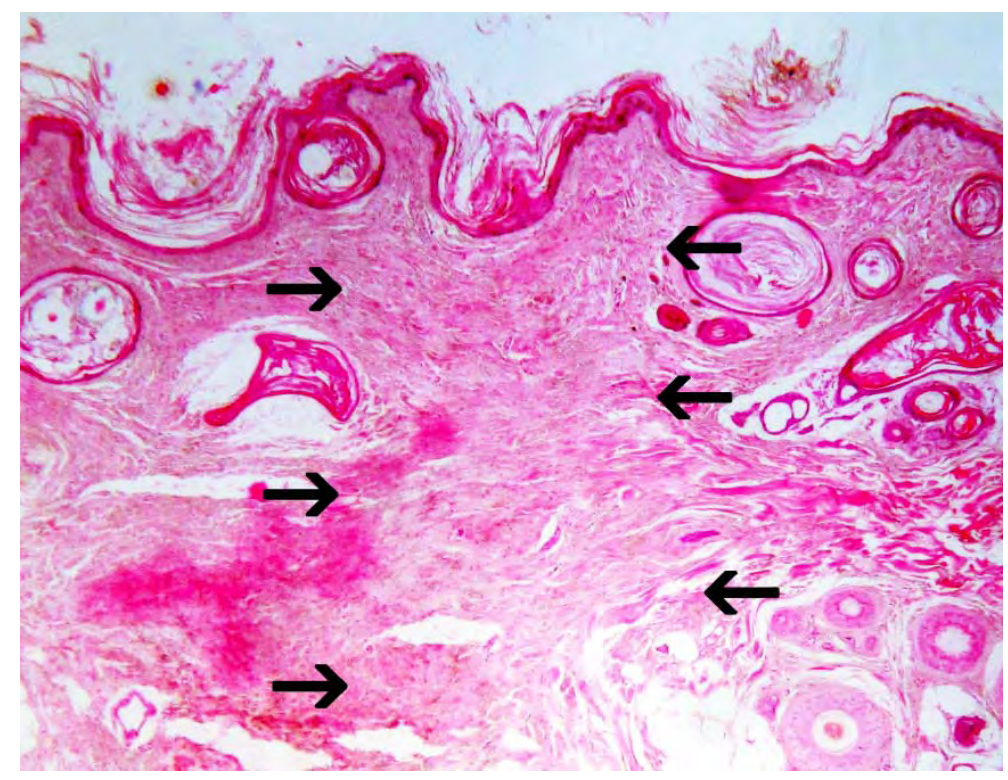

Figure 40. Wound area at the site of the incision (solid arrows) on the $365^{\text {th }}$ po.d, without any glands or hair follicles, and with thinner than normal bands of collagen. 


\section{Skin glue}

On the $7^{\text {th }}$ po.d., the main finding was the infiltration of the wound area at the site of the incision by neutrophils, macrophages, lymphocytes, and fibroblasts (Figure 41). The thickness of the epidermis was increased $x$ 2-6 times compared to the normal adjacent epidermis (Figures 41, 42). The upper dermis next to the wound area was infiltrated by macrophages, neutrophils, lymphocytes and a few mast cells. In two animals, epidermis gap was noticed (Figure 43a-c). At the wound area, the collagen fibers were thinner compared to those in the nearby area (Figure 43d). Foci with traumatic furunculosis were found (B1, B3, B7) (Figure 43a,b). Microscopic analysis showed that, in spite of care during application, small quantities of glue penetrated the wound, initiating inflammation of the dermis in two animals (B3, B7) (Figure 44a,b).
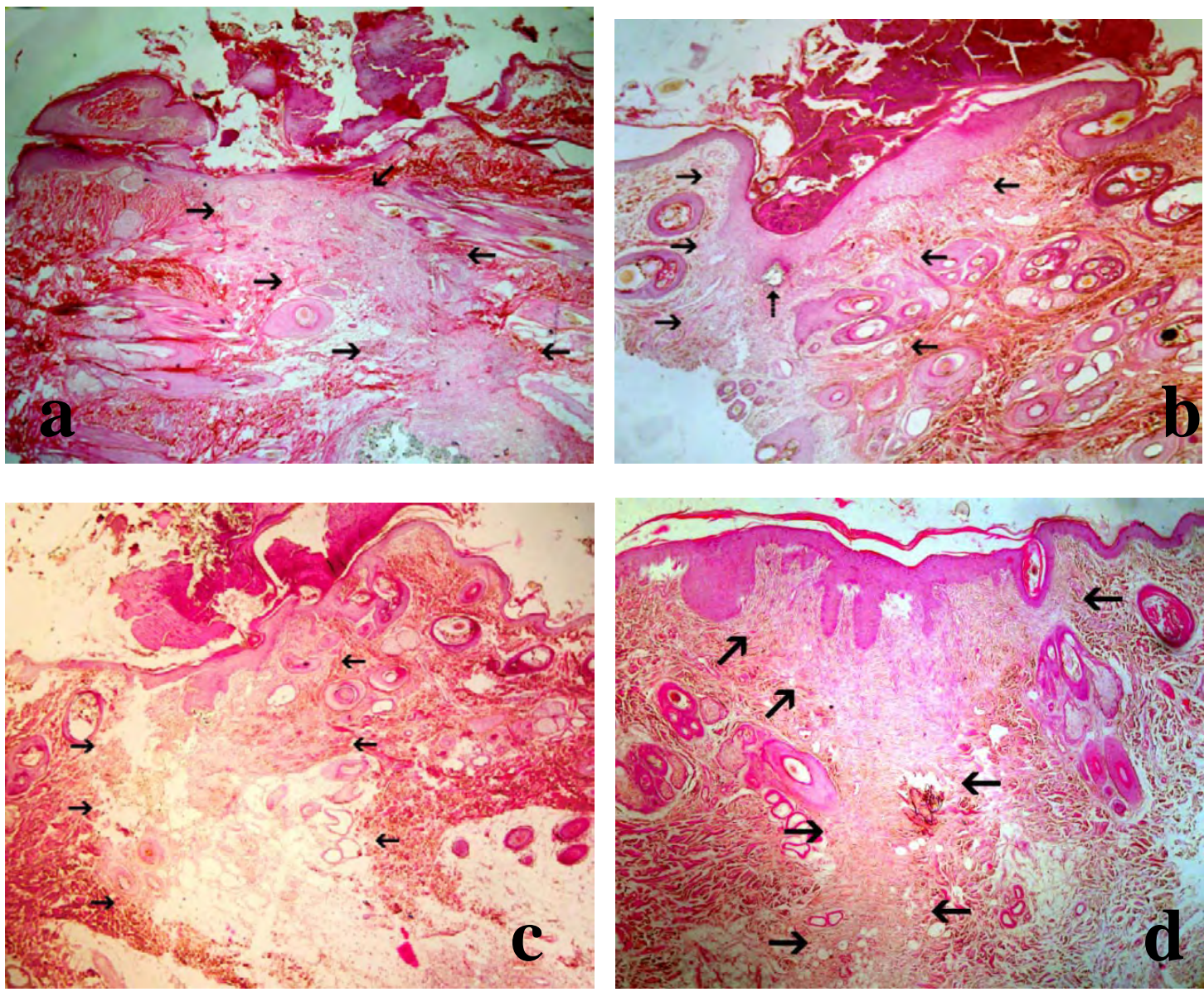

Figure 41a,b,c,d. Wound area at the site of the incision (solid arrows), infiltrated by neutrophils, macrophages, lymphocytes, and fibroblasts. b. Glue penetrating the wound (dotted arrow). 


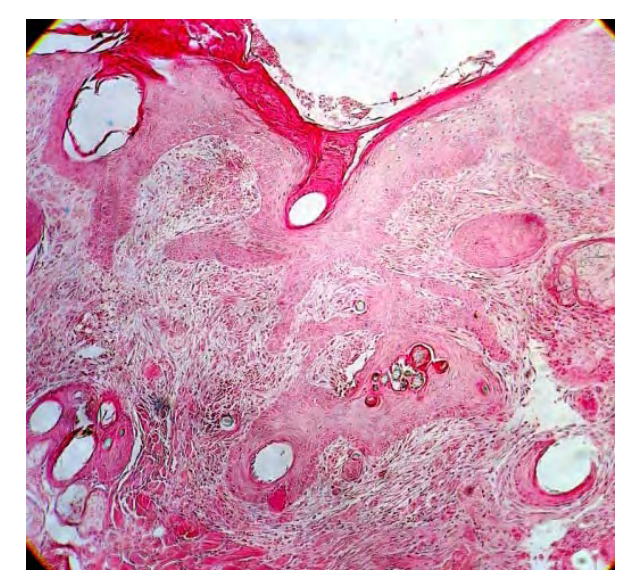

Figure 42. Epidermis of increased thickness at the site of the incision.
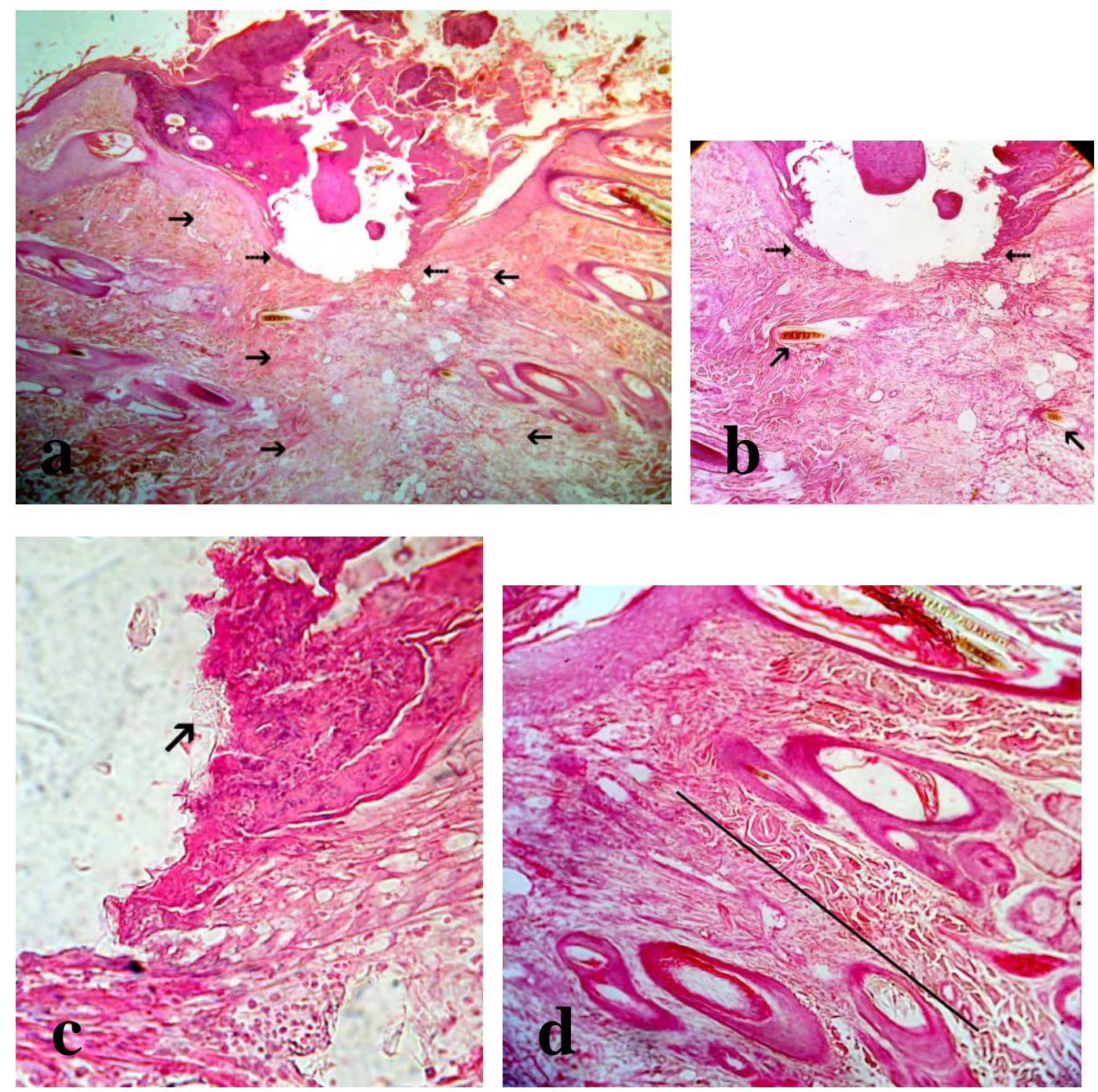

Figure 43a,b. Wound area (between arrows) at the site of the incision on the $7^{\text {th }}$ po.d., where epithelial bridging has not been completed. c. Glue substance at the borders of the epithelium. d. Thinner collagen fibers at the wound area (on the left side of the black line) compared to the collagen fibers of the nearby normal skin which is on the right side of the line. 

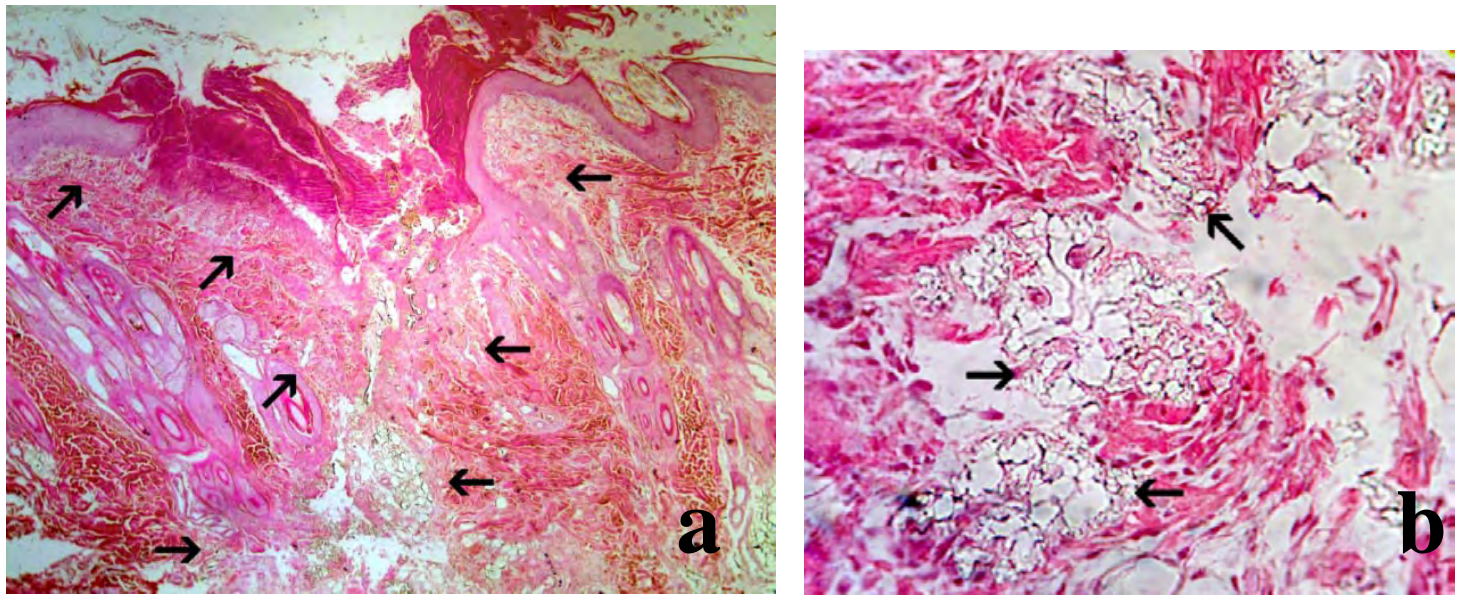

Figure 44a,b. Wound area (a, arrows) at the site of the incision on the $7^{\text {th }}$ po.d. Small quantities of glue (arrows) penetrated the wound, initiating inflammation of the dermis (b).

On the $14^{\text {th }}$ po.d., the main finding was the infiltration of the wound area at the site of the incision by fibroblasts and macrophages (Figure 45a,b). The thickness of the epidermis was increased $\times$ 2-8 times compared to the normal epidermis, showing hyperplasia in one animal (B3) (Figure 45b). The upper dermis next to the wound area was infiltrated by macrophages and mast cells. One large area with hair fragments and intense inflammatory reaction, with macrophages and neutrophils, was observed in the subcutaneous tissue of one animal (B3) (Figure 46a-c). Small quantities of glue inside the dermis, initiating inflammation, were observed in two animals (B2, B3) (Figure 47a-d). One comedo was found in one animal (B4) (Figure 48a,b).
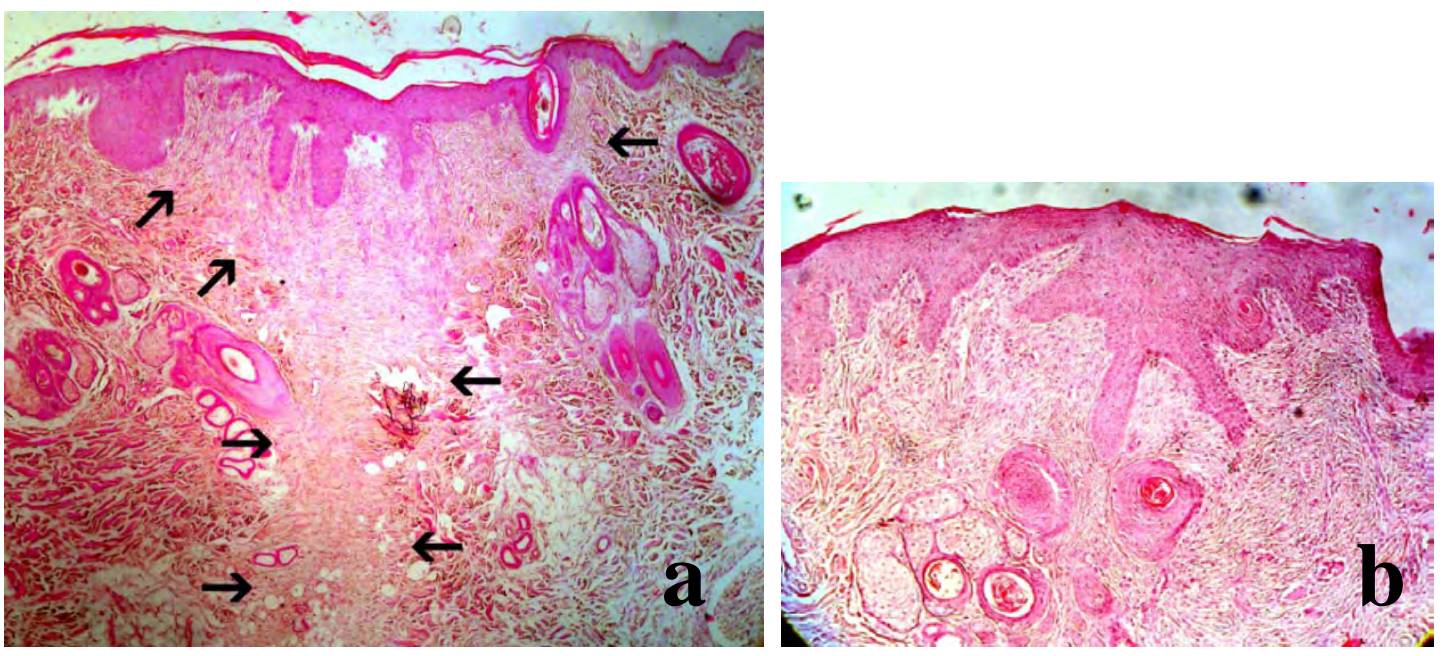

Figure 45a,b. Wound area at the site of the incision (between arrows) on the $14^{\text {th }}$ po.d, with intense thickening of the epidermis. 

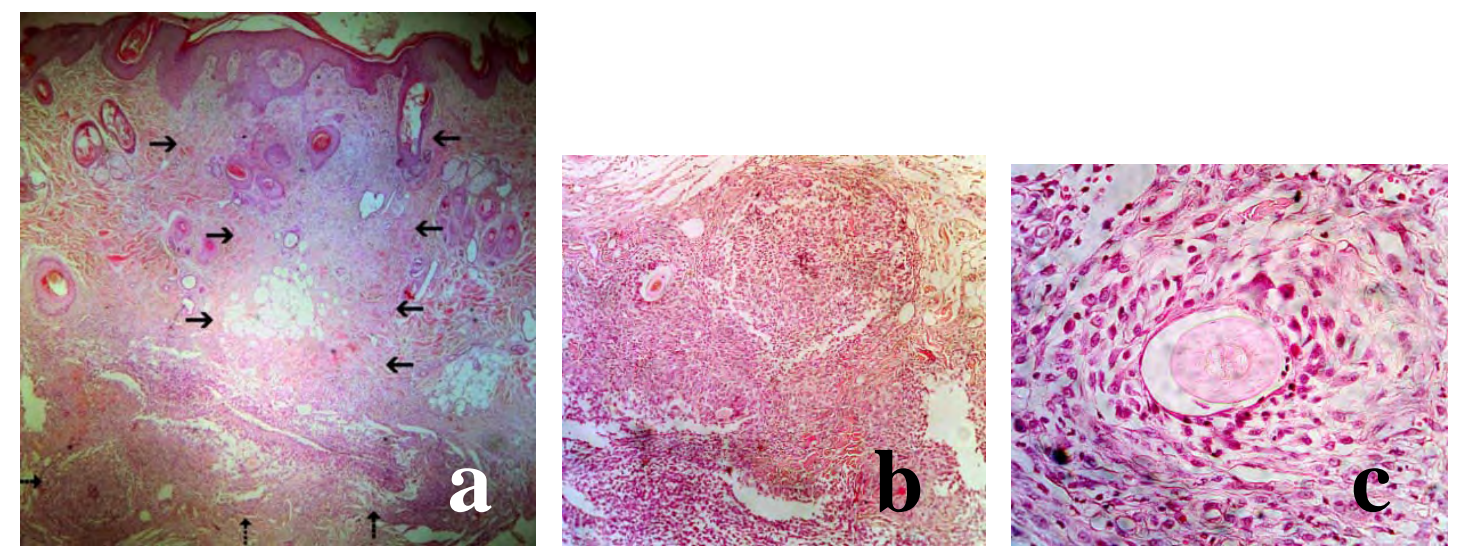

Figure 46a-c. Wound area at the site of the incision on the $14^{\text {th }}$ po.d. with a large area with hair fragments and intense inflammatory reaction with macrophages and neutrophils in the subcutaneous tissue (dotted arrows, b,c)
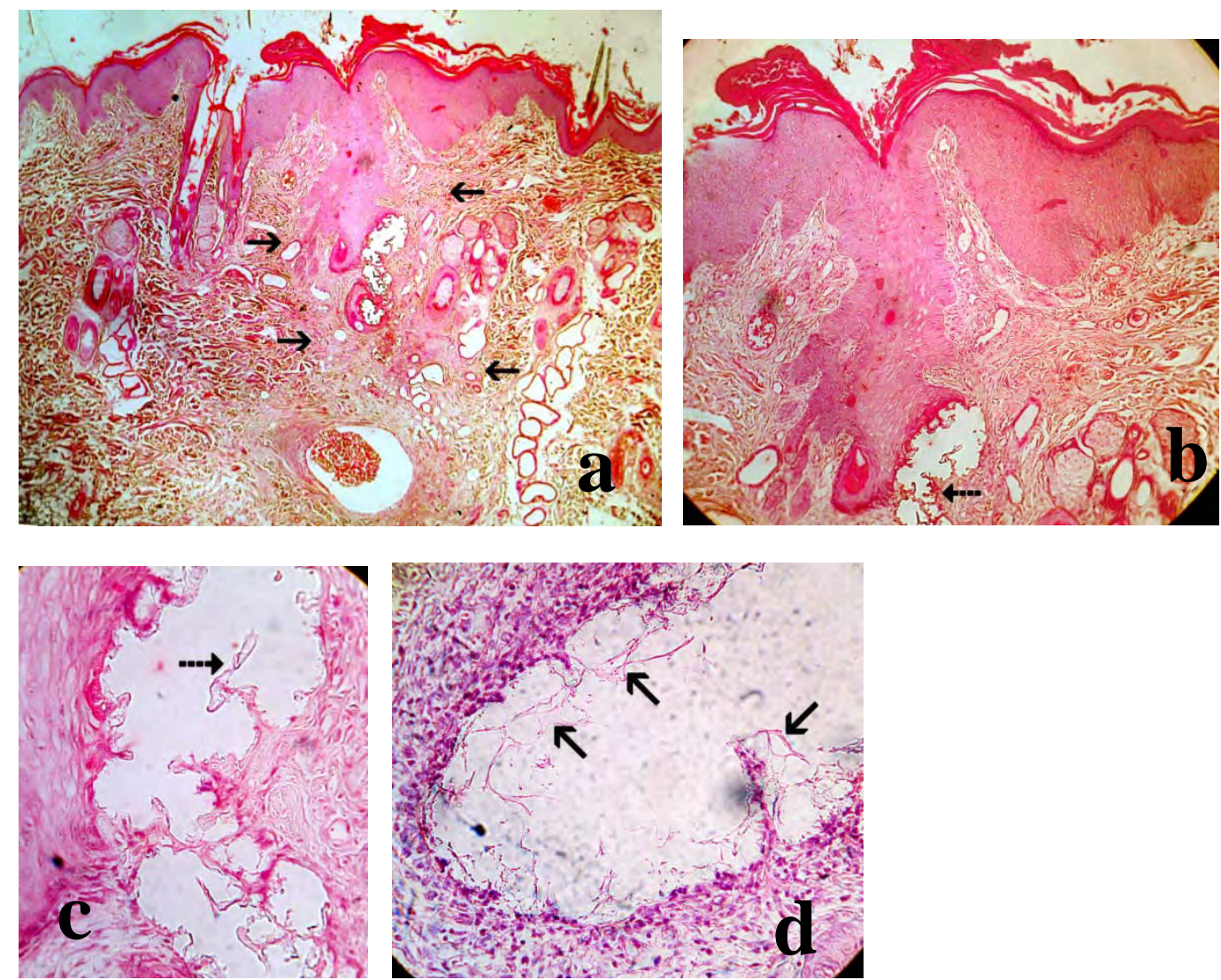

Figure 47a-d. Wound area at the site of the incision (between arrows) on the $14^{\text {th }}$ po.d. b. Intense epithelial thickening and glue substance at wound area (dotted arrow). c. Glue substance (arrows) inside dermis initiating inflammatory reaction (d). 

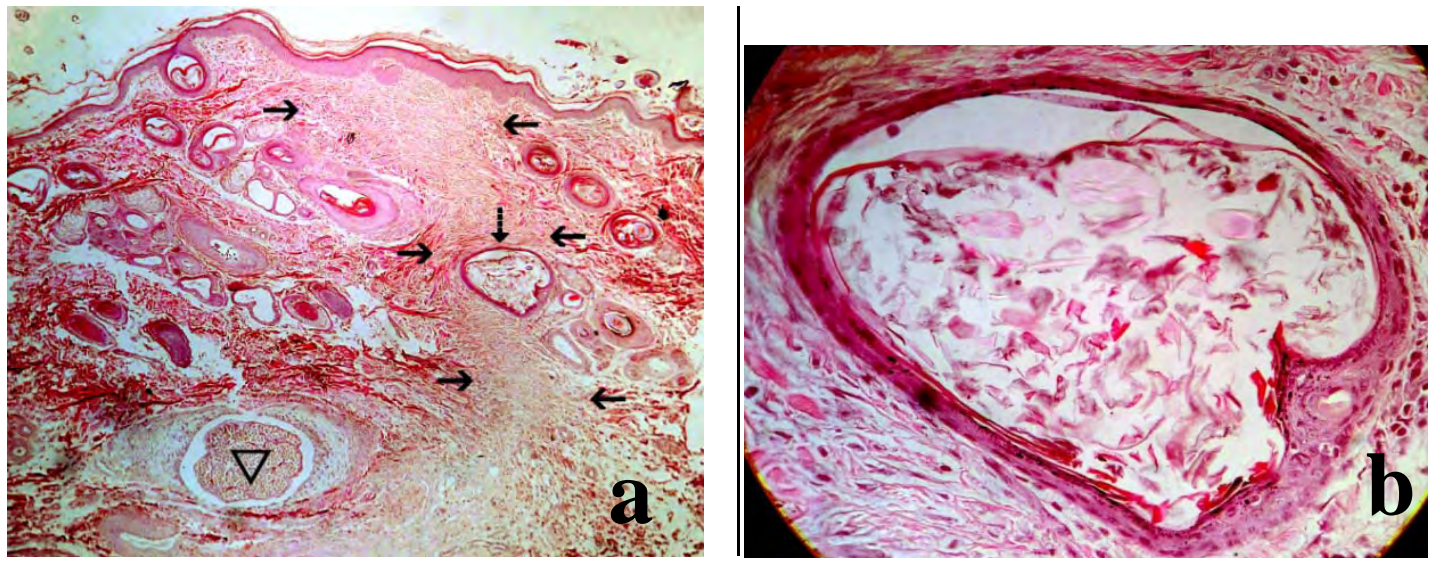

Figure 48a,b. Wound area at the site of the incision (between black arrows) on the $14^{\text {th }}$ po.d. A closed comedo is seen (dotted arrow, b) and the multifilament suture (triangle) that was used for subcutaneous suturing.

On the $\mathbf{2 8}^{\text {th }}$ po.d., the main finding was the absence of glands or hair follicles at the wound area, with thinner than normal bands of collagen and infiltration by small to medium numbers of macrophages and lymphocytes (Figure 49a,b). The upper dermis next to the wound area was infiltrated by a few macrophages, lymphocytes and mast cells (B5) (Figure 50a,b). The thickness of the epidermis had increased x1.5-2.5 times compared to the normal adjacent epidermis, whilst in one dog (B2) pseudocarcinomatous hyperplasia was found (Figure 51). Small quantities of glue inside the dermis, initiating inflammation, were found in 2 animals (B2, B3) (Figure 52 a-e). Foci with traumatic furunculosis and a comedo (B7) were found in one animal (Figure 53) and areas with giant multinucleated cells around glue substance were observed in another animal (B8) (Figure 54a,b).
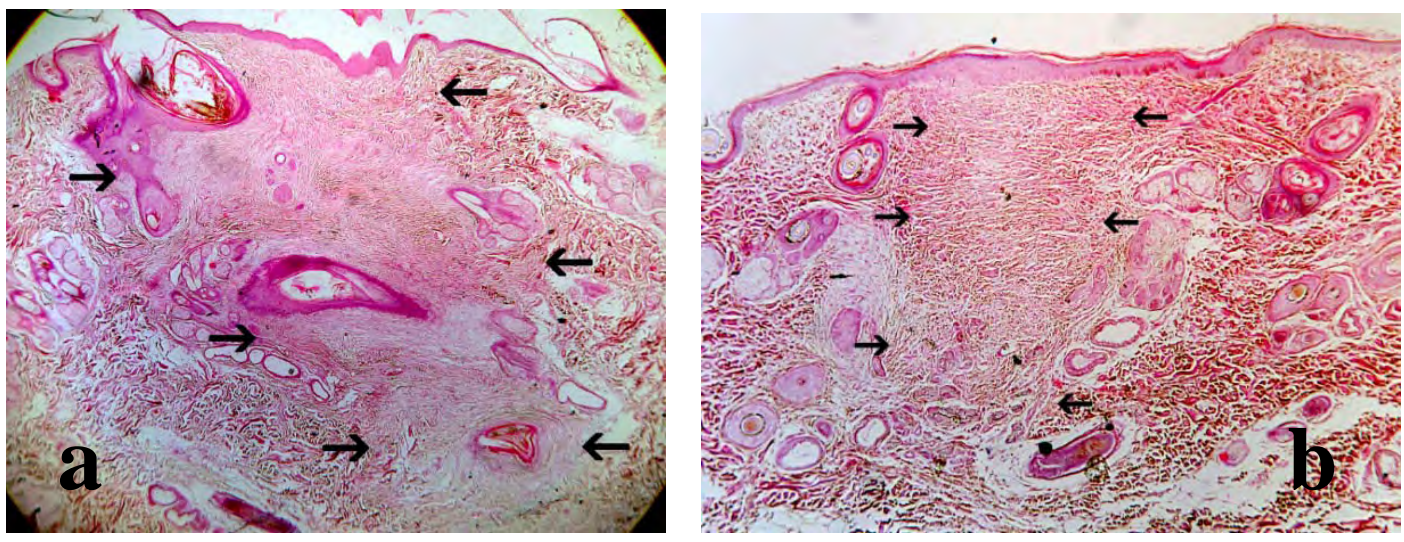

Figure 49a,b. Wound area at the site of the incision (between arrows) on the $28^{\text {th }}$ po.d., without any glands or hair follicles, and with thinner than normal bands of collagen. 

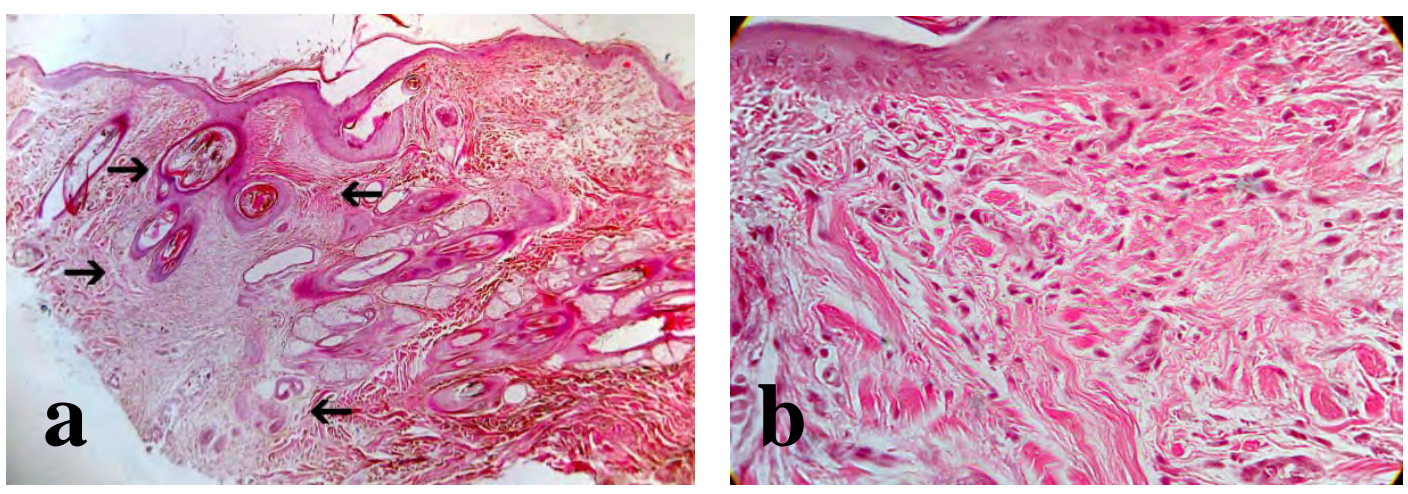

Figure 50a,b. Wound area at the site of incision (black arrows) on the $28^{\text {th }}$ po.d with leukocytes infiltration of the upper dermis next to the wound area (b).

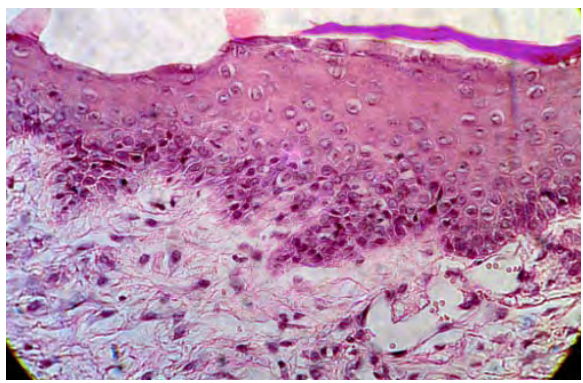

Figure 51. Pseudocarcinomatous hyperplasia of the epidermis at the incision site on the $28^{\text {th }}$ po.d.
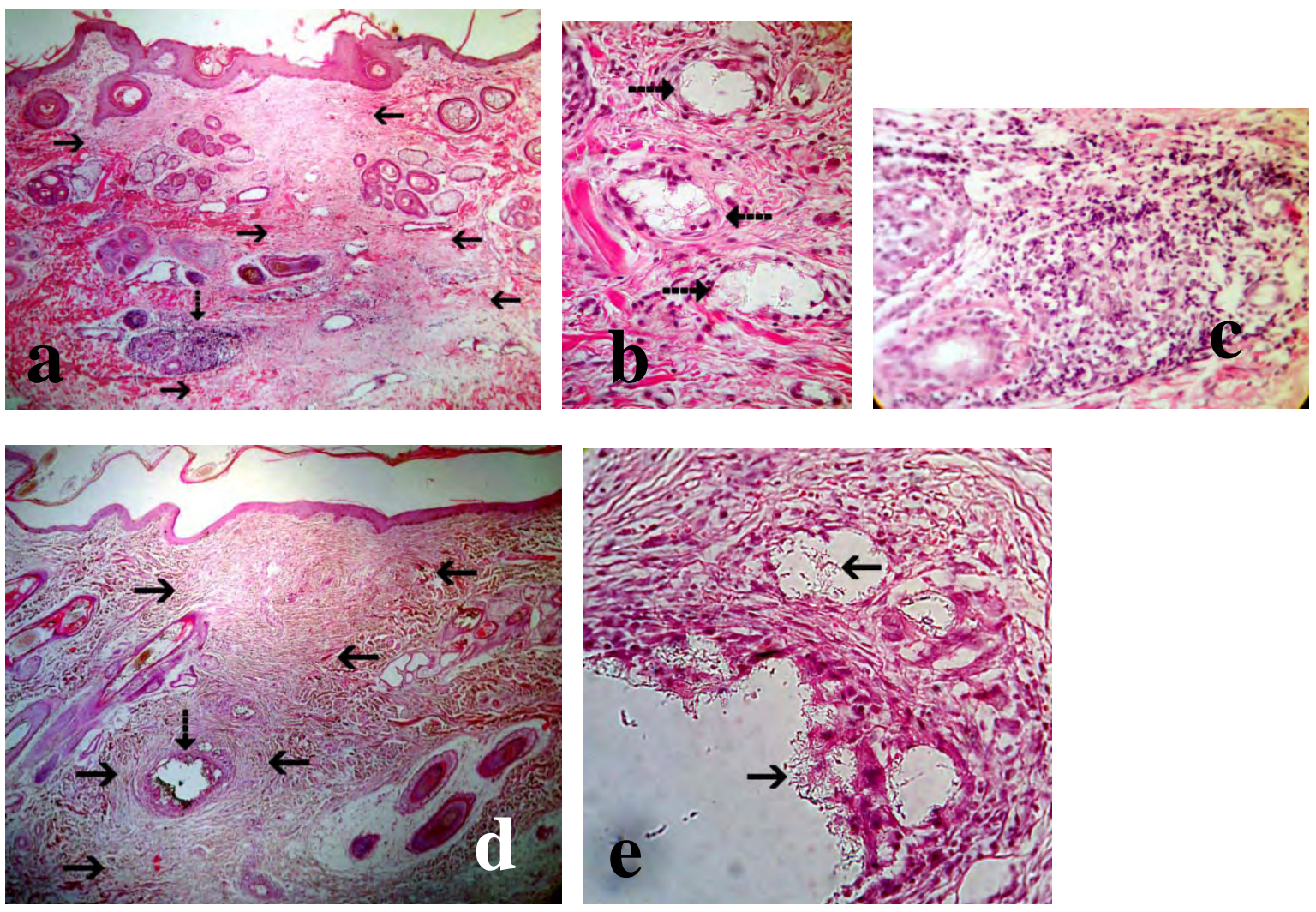

Figure 52 a-e. Wound area at the site of the incision (a, d, arrows) on the $28^{\text {th }}$ po.d. with areas of inflammatory reaction (a, b, dotted arrows). b, c, e. Areas with glue substance (arrows) inside the dermis, initiating inflammatory reaction. 


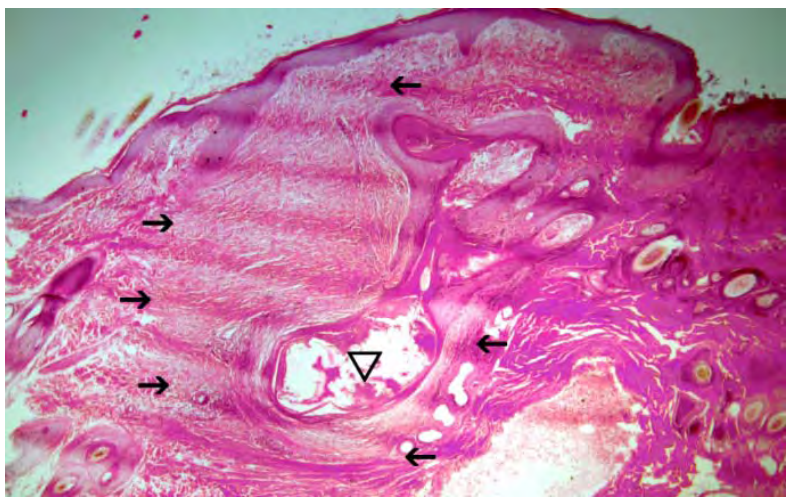

Figure 53. Wound area at the site of the incision (arrows), on the $28^{\text {th }}$ po.d., with a closed comedo (triangle).
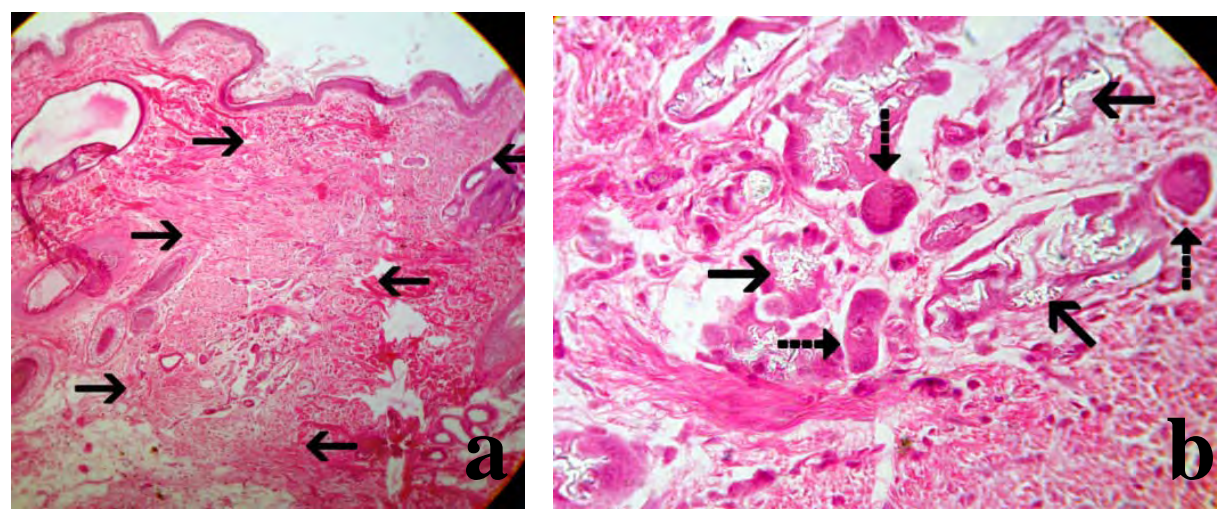

Figure 54a,b. Wound area at the site of incision (black arrows) on the $28^{\text {th }}$ po.d. with areas with giant multinucleated cells around glue substance (b).

On the $180^{\text {th }}$ po.d. , the main finding was the absence of glands or hair follicles at the wound are, with thinner than normal bands of collagen (Figure 55a-d). In one animal (B8), the dermis at the wound area was infiltrated by few macrophages, mast cells, and lymphocytes (Figure 56a,b). The thickness of the epidermis was increased x1-1.5 times compared to the normal adjacent epidermis. One comedo (Figure 57) was found in one animal (B9). Hair fragments initiating inflammation were seen inside the wound area.
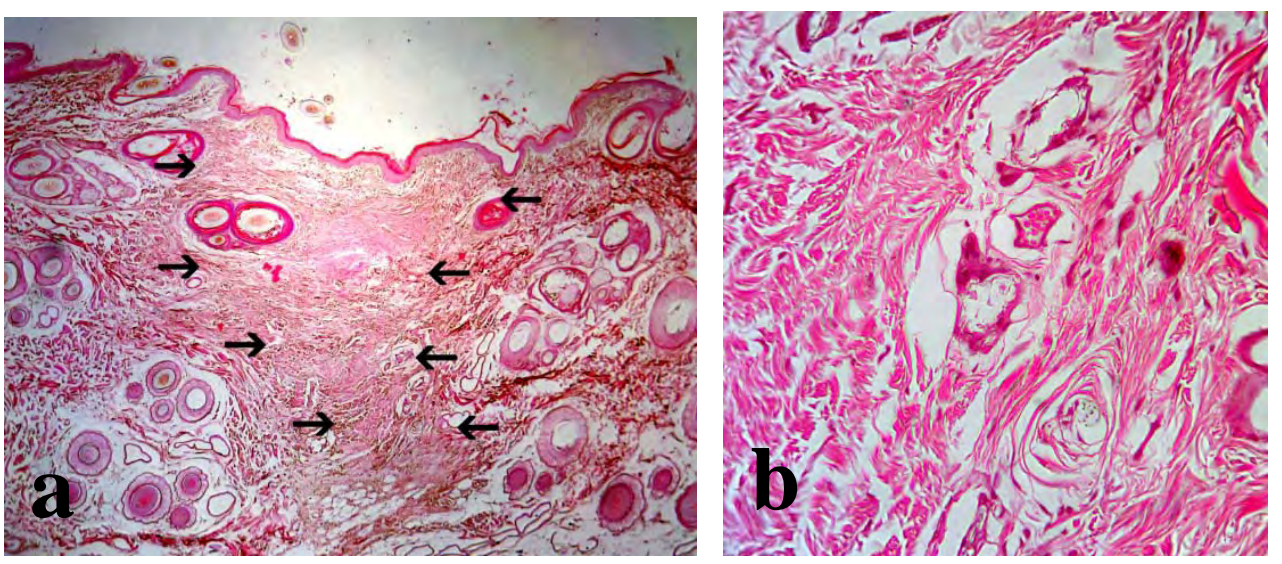

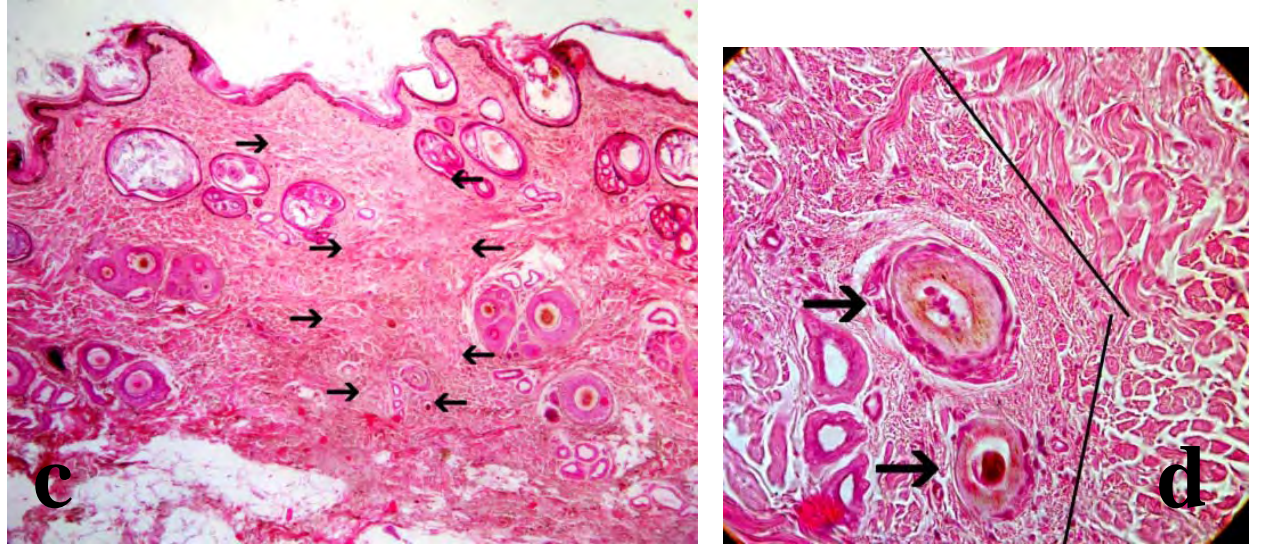

Figure 55a-c. Wound area at the site of incision (between arrows) without any glands and hair follicles and with thinner than normal bands of collagen (b, d- at the right of the black lines).
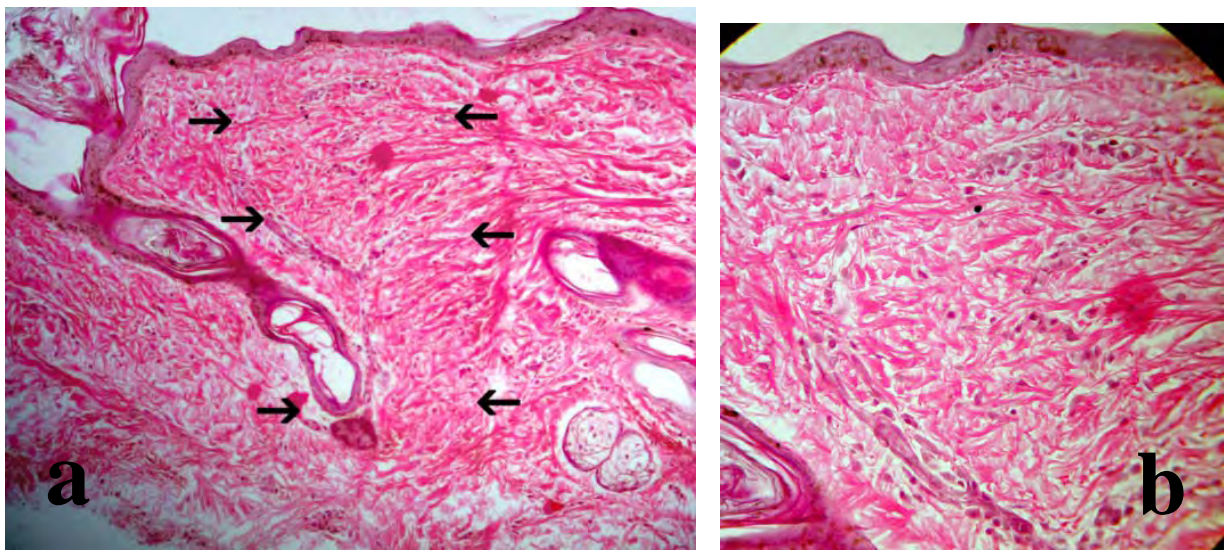

Figure 56a,b. Wound area at the site of the incision on the $180^{\text {th }}$ po.d, with thinner than normal bands of collagen and mild infiltration by few macrophages, mast cells and lymphocytes.

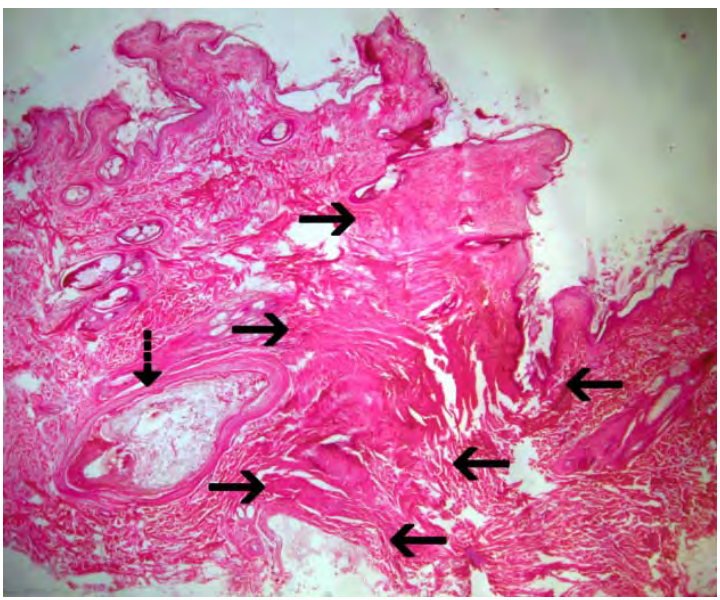

Figure 57. Wound area at the site of incision (between arrows) on the $180^{\text {th }}$ po.d. with a closed comedo (dotted arrow). 
On the $\mathbf{3 6 5}^{\text {th }}$ po.d., the main finding was the absence of glands or hair follicles from the wound area, where thinner than normal collagen fiber bands were observed. The thickness of the epidermis was increased x1-2.5 times compared to the normal adjacent epidermis (Figure 58a,b). Small quantities of glue inside dermis, initiating inflammation, were found in two animals (Figure 59a-d).
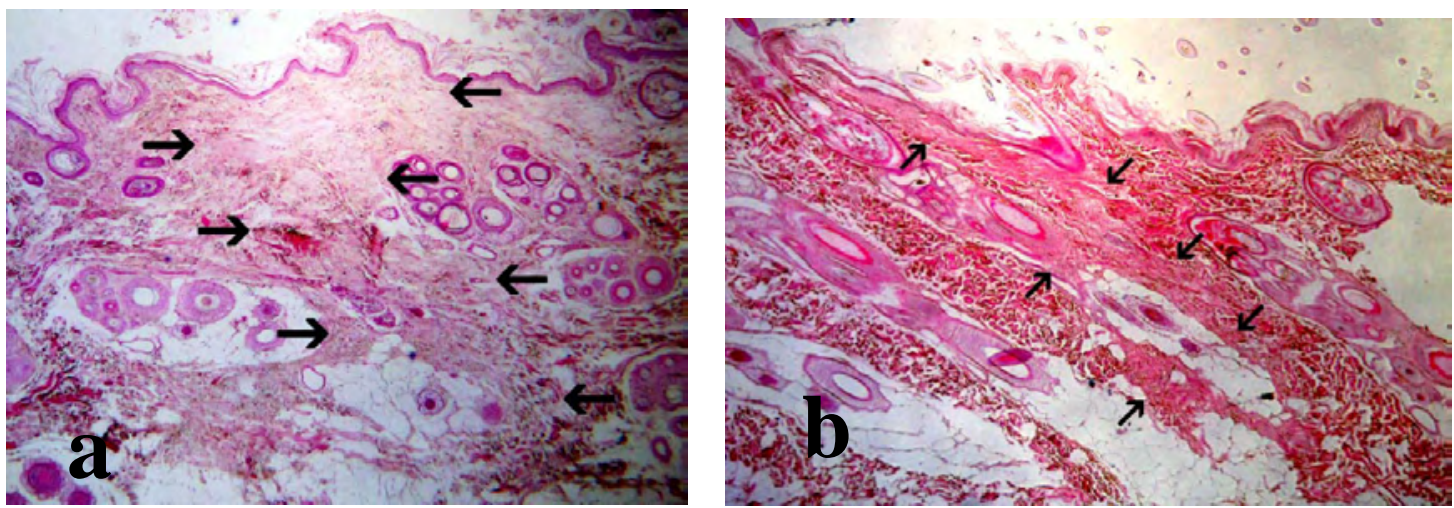

Figure 58a,b. Wound area at the site of incision (between arrows) on the $365^{\text {th }}$ po.d.; absence of glands or hair follicles, and presence of thinner than normal collagen fiber bands.
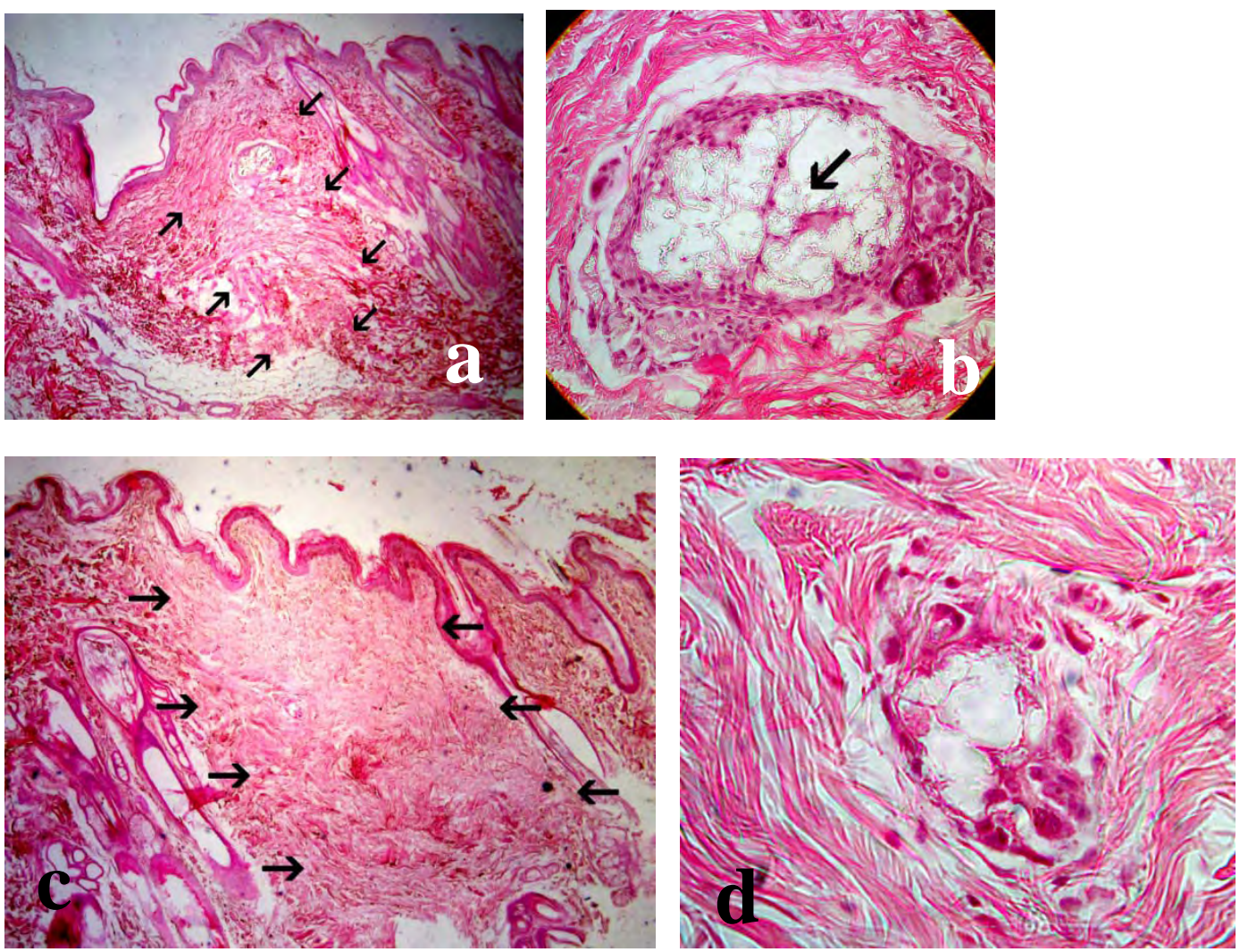

Figure 59a, c. Wide wound area at the site of the incision (between arrows) on the $365^{\text {th }}$ po.d; absence of glands or hair follicles, and thinner than normal collagen fiber bands are evident. b, d. Small quantities of glue inside the dermis, initiating inflammation. 


\section{Intradermal suture pattern with burying of the knot}

On the $7^{\text {th }}$ po.d., the main finding was infiltration of the wound area at the site of the incision by a medium number of neutrophils, macrophages, and fibroblasts (Figures 60-62). Minimal to medium tissue reaction, composed of macrophages and fibroblasts, was usually observed around suture material. The thickness of the epidermis was increased x 2-3 times compared to the normal adjacent epidermis, whilst in one case it was increased x 5 times (Figure 61b). The upper dermis next to the wound area was infiltrated by small numbers of macrophages and few mast cells. In one animal (B7) there was an accumulation of macrophages and lymphocytes in the subcutaneous tissue (nodular inflammation).
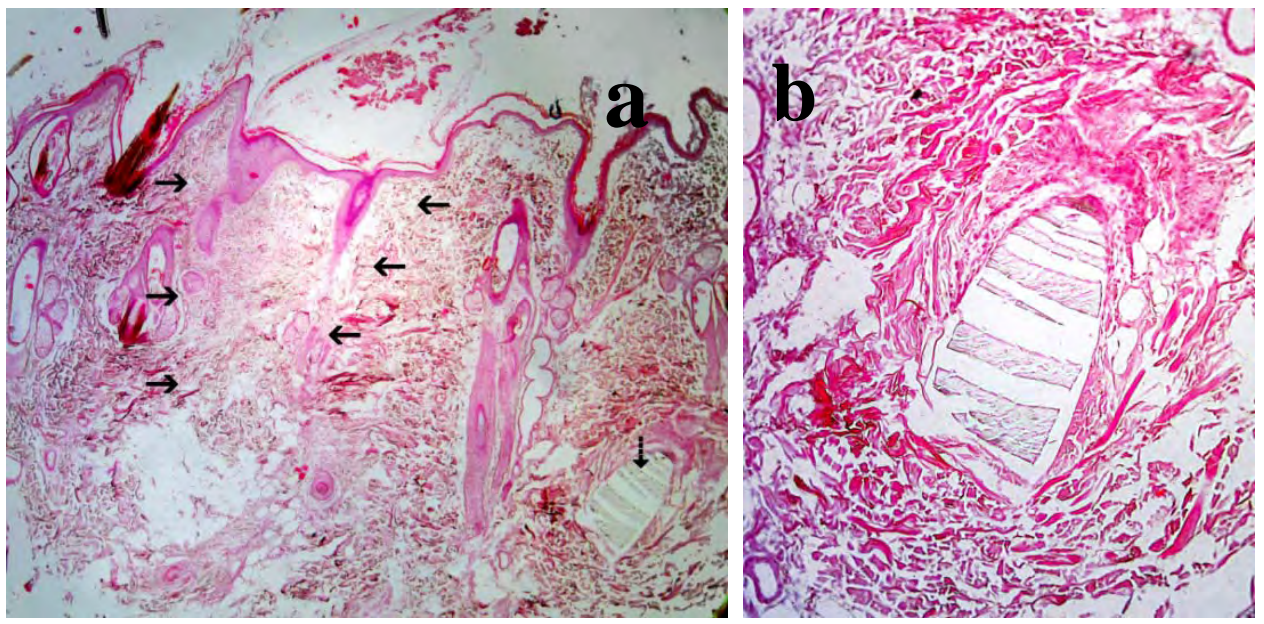

Figure 60a. Wound area at the site of incision (black arrows) on the $7^{\text {th }}$ po.d., infiltrated by neutrophils, macrophages, and fibroblasts. b. Minimal tissue reaction, composed of macrophages and fibroblasts, observed around suture material.
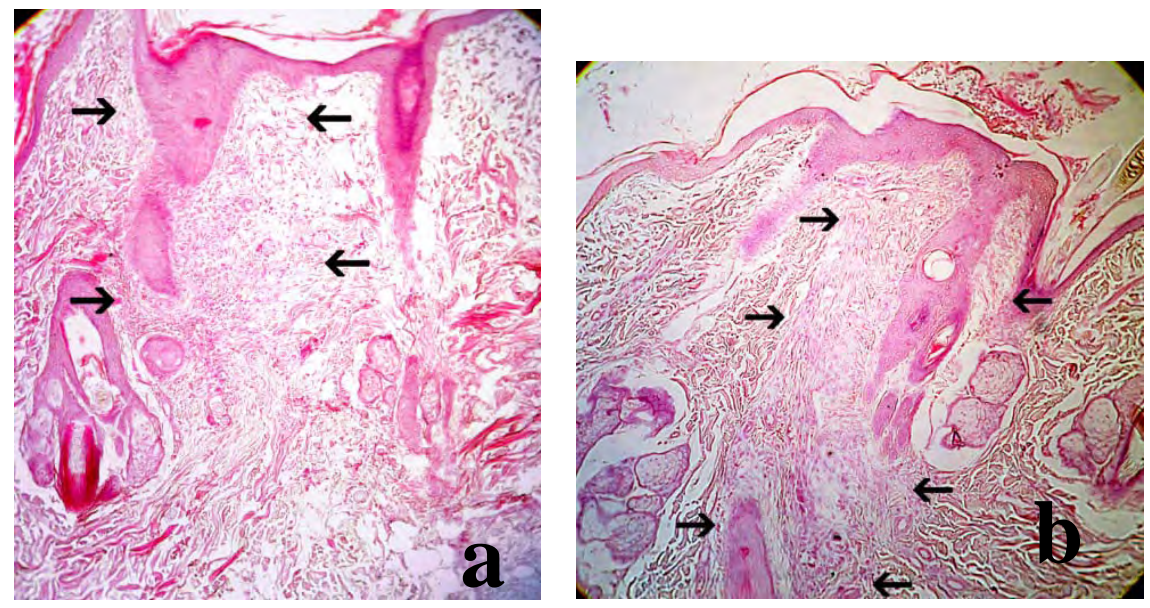

Figure 61a,b. Wound area at the site of incision (black arrows) on the $7^{\text {th }}$ po.d., infiltrated by neutrophils, macrophages, and fibroblasts. 


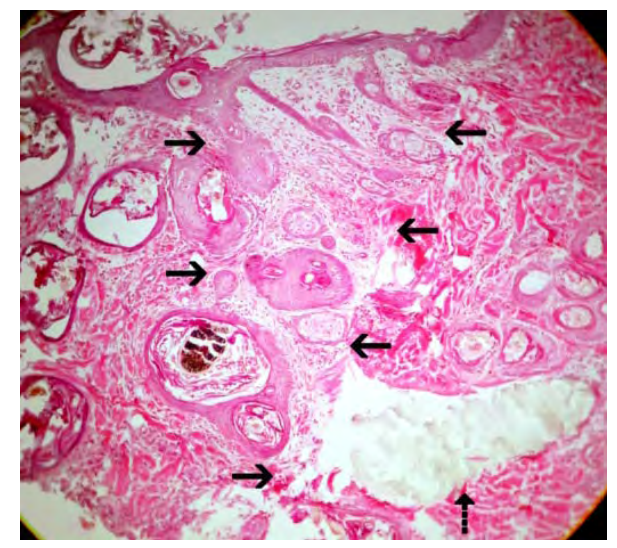

Figure 62. Wound area at the site of incision (black arrows) on the $7^{\text {th }}$ po.d., infiltrated by neutrophils, macrophages, and fibroblasts. Suture material without inflammatory tissue reaction around it (dotted arrow)

On the $14^{\text {th }}$ po.d., the main finding was infiltration of the wound area at the site of the incision by a large to medium number of neutrophils, macrophages, and fibroblasts. Minimal to medium tissue reaction, composed of macrophages and fibroblasts, was usually observed around the suture material. The thickness of the epidermis was increased x 1.5-3 times compared to the normal adjacent epidermis (Figures 63-65). In one animal the thickness of the epidermis was even larger (Figure 67, triangle).
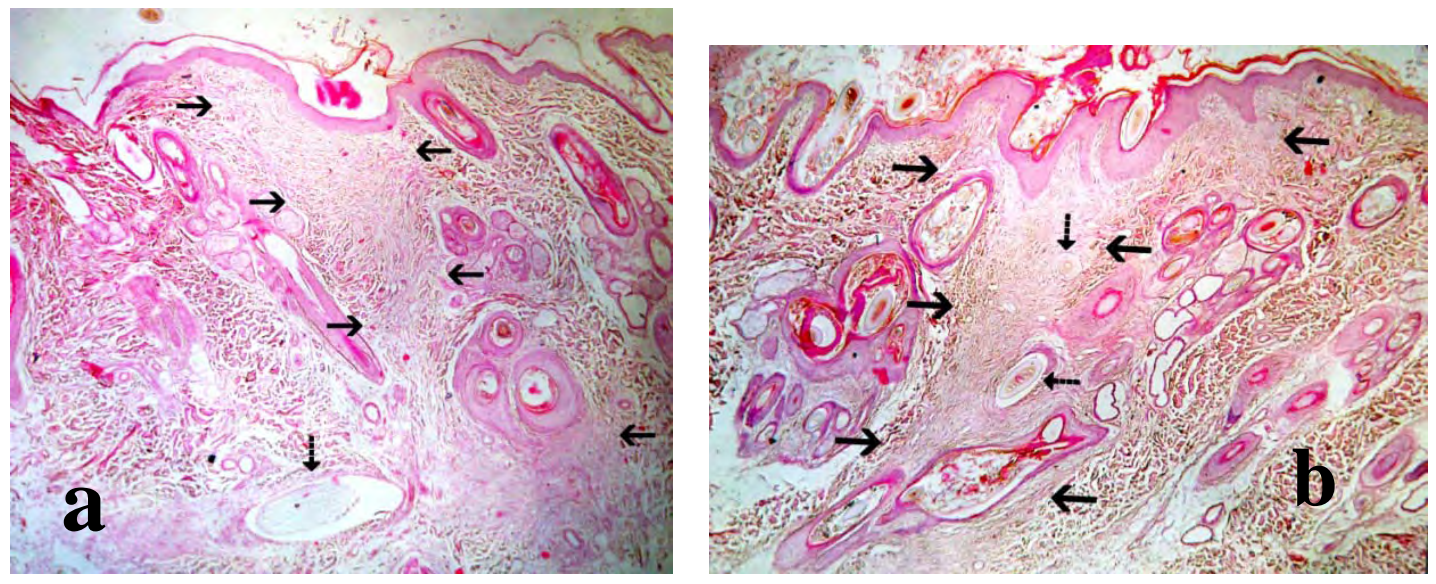

Figure 63a,b. Wound area at the site of the incision on the $14^{\text {th }}$ po.d. Minimal tissue reaction observed around suture material (a, dotted arrow). Hair fragments, initiating inflammatory reaction, noticed inside the wound area (b, dotted arrows) 

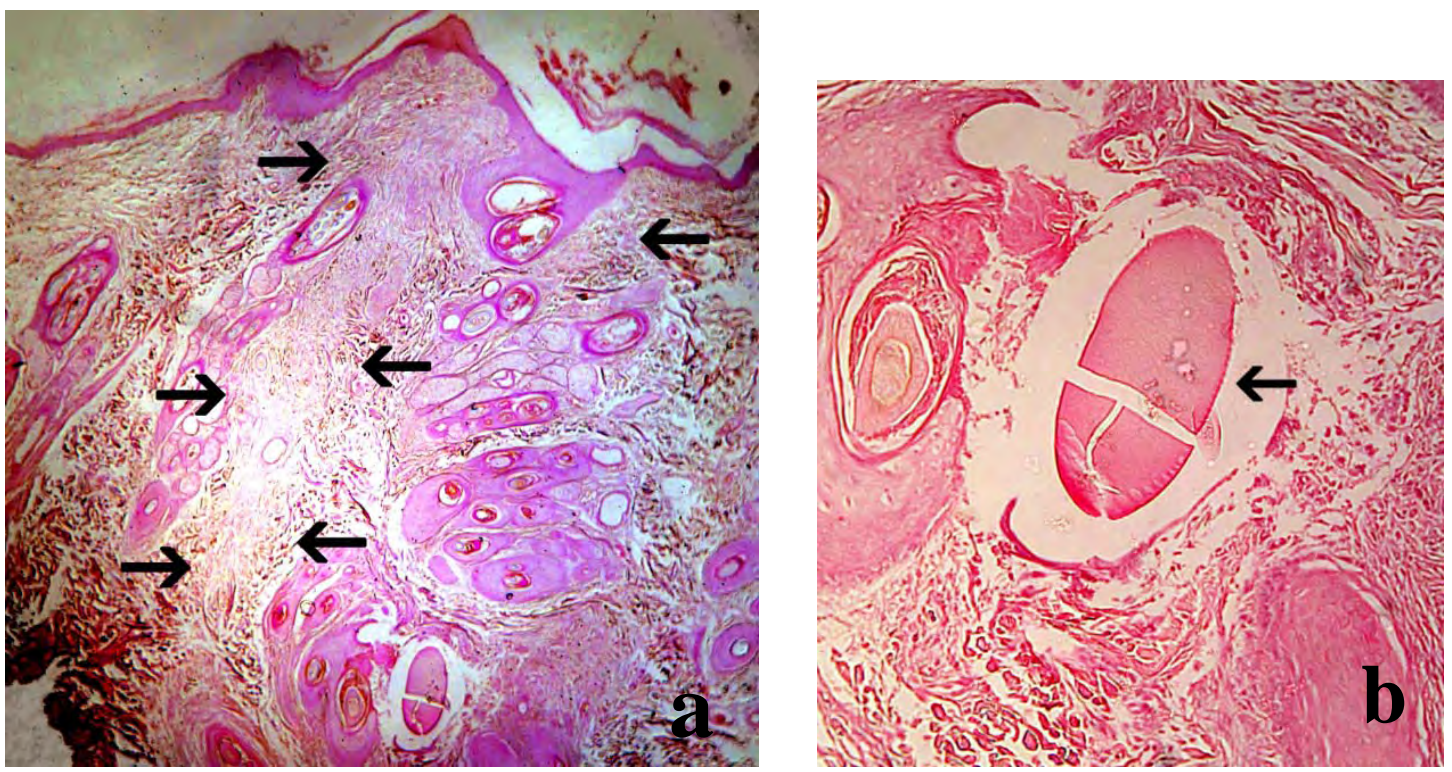

Figure 64a. Wound area (arrows) at the site of the incision on the $14^{\text {th }}$ po.d. $\mathbf{b}$. Minimal tissue reaction observed around suture material (arrow).

The upper dermis next to the wound area was infiltrated by small numbers of macrophages and minimal numbers of mast cells. In two animals (B4, B7) the histological examination showed nodular accumulation of macrophages and lymphocytes in the subcutaneous tissue and a closed comedo (B4) (Figure 65a,b). In one animal (B9), two foci with traumatic furunculosis were found (Figure 67a,b).
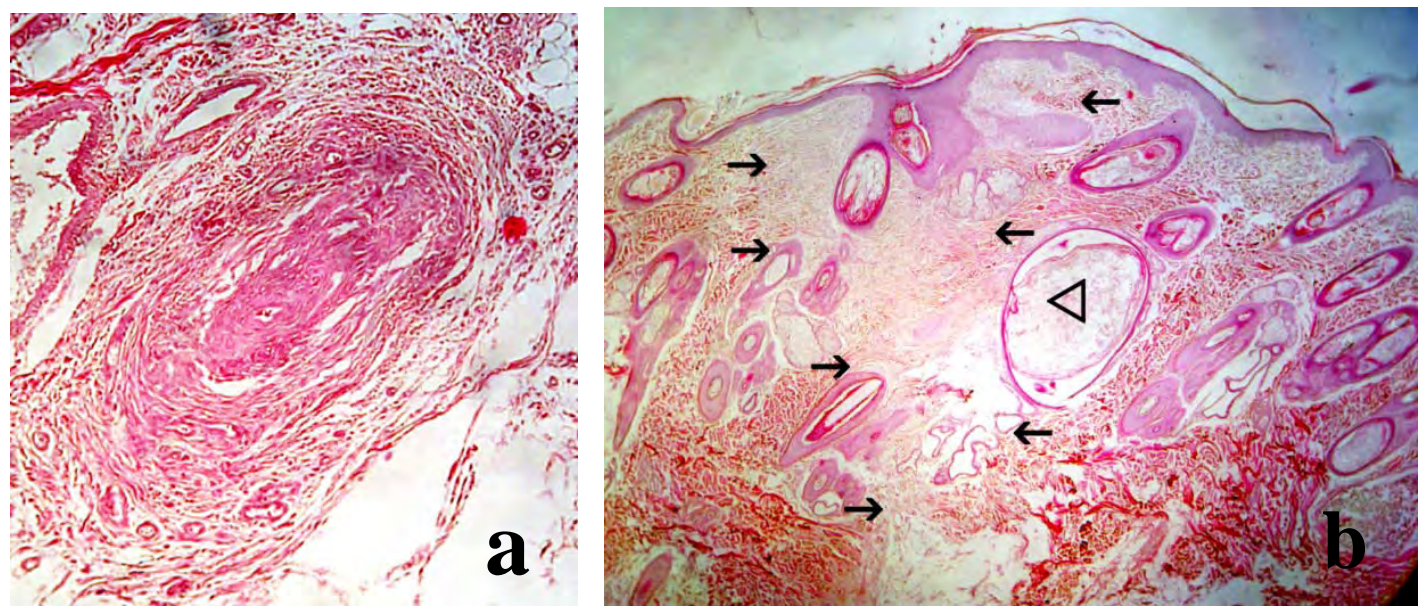

Figure 65a. Nodular accumulation of macrophages and lymphocytes in the subcutaneous tissue on the $14^{\text {th }}$ po.d. b. Wound area at the site of incision (black arrows) and a closed comedo (triangle). 

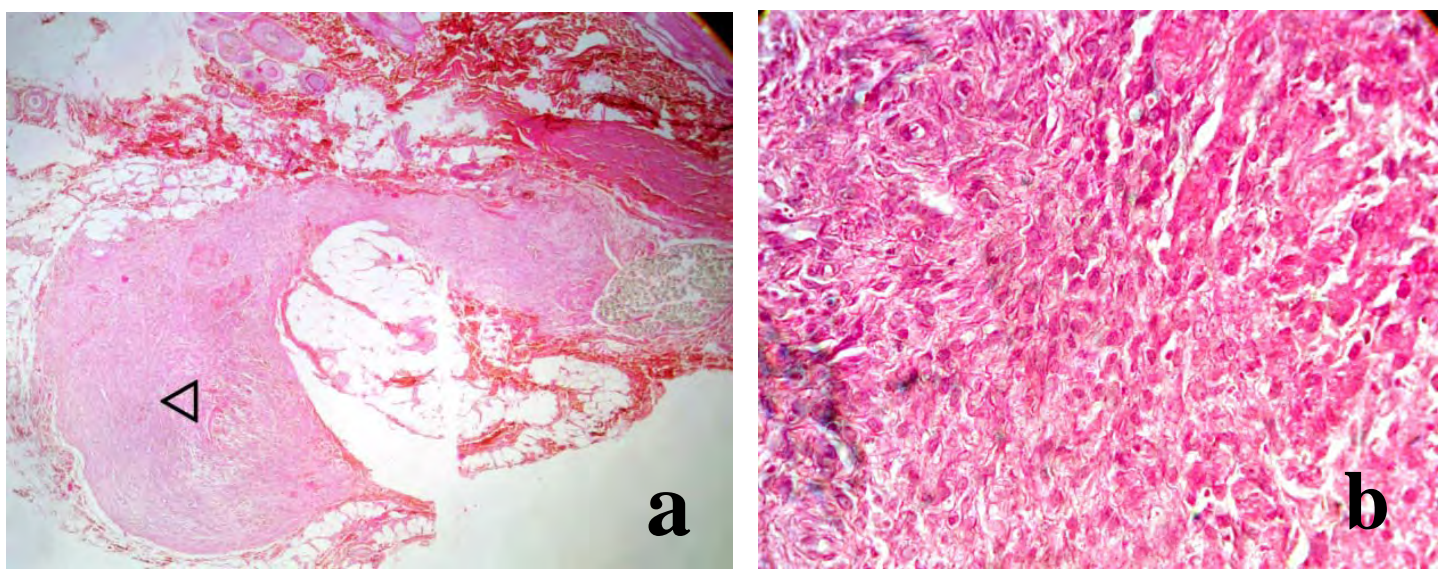

Figure 66a,b. Large nodular accumulation of macrophages and lymphocytes in the subcutaneous tissue on the $14^{\text {th }}$ po.d. (triangle).
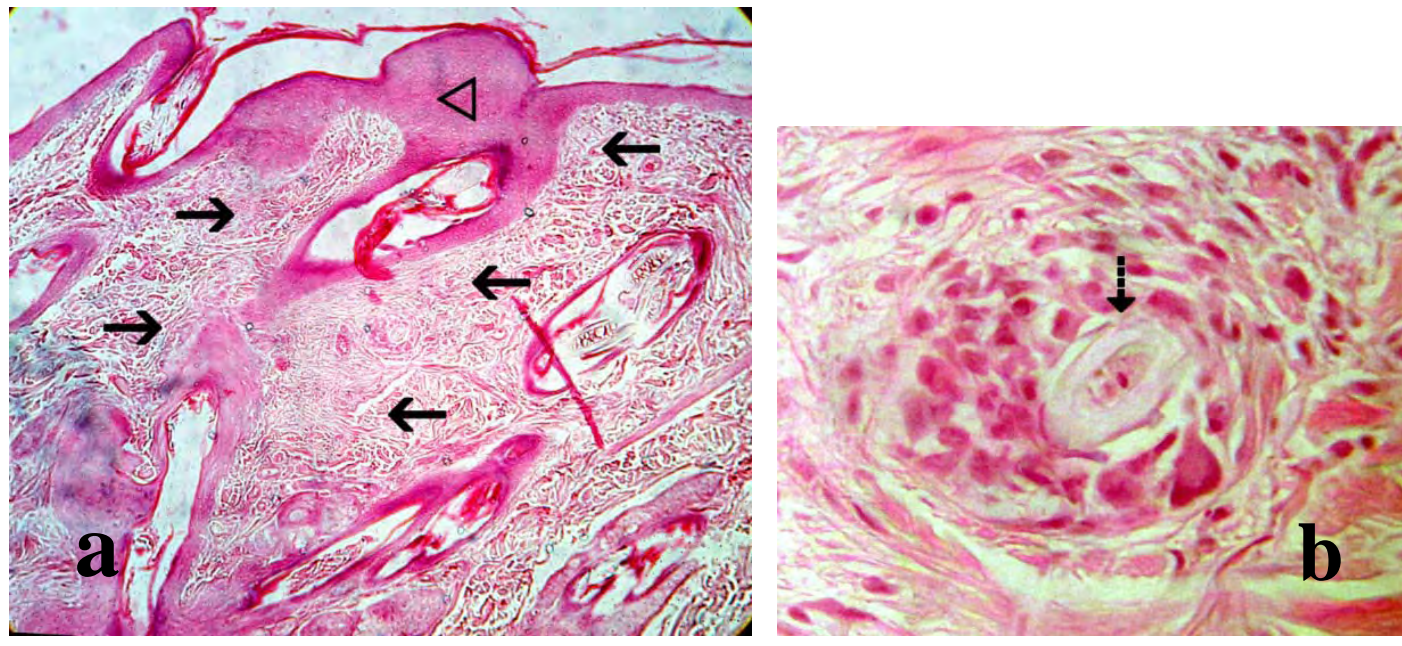

Figure 67a,b. Wound area at the site of incision (black arrows) on the $14^{\text {th }}$ po.d. with hair fragments initiating inflammatory reaction $\mathbf{b}$. The thickness of the epidermis is much larger than normal (triangle in a).

On the $28^{\text {th }}$ po.d., the main finding was the absence of glands and hair follicles from the wound area at the site of incision, and the presence of thinner than normal collagen fiber bands and a small number of macrophages and lymphocytes (Figure 68a,b).

Minimal to medium tissue reaction, composed of macrophages and fibrosis, was usually observed around the suture material (Figure 69a,b). 

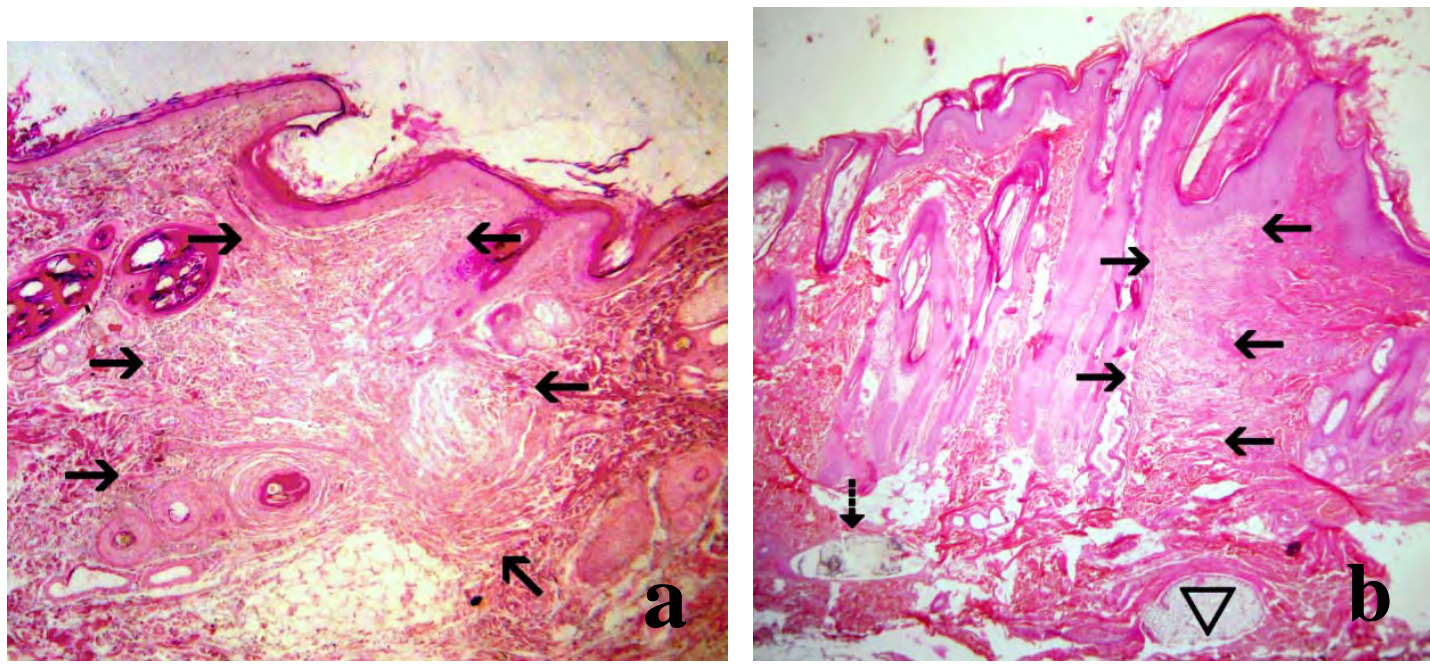

Figure 68a. Wide wound area at the site of incision (arrows), without any glands or hair follicles, and with thinner than normal collagen fiber bands, on the $28^{\text {th }}$ po.d. $\mathbf{b}$. Thin wound area (arrows). Poliglecaprone 25 suture material with minimum reaction (dotted arrow) and multifilament suture material (triangle) in the subcutaneous tissue.
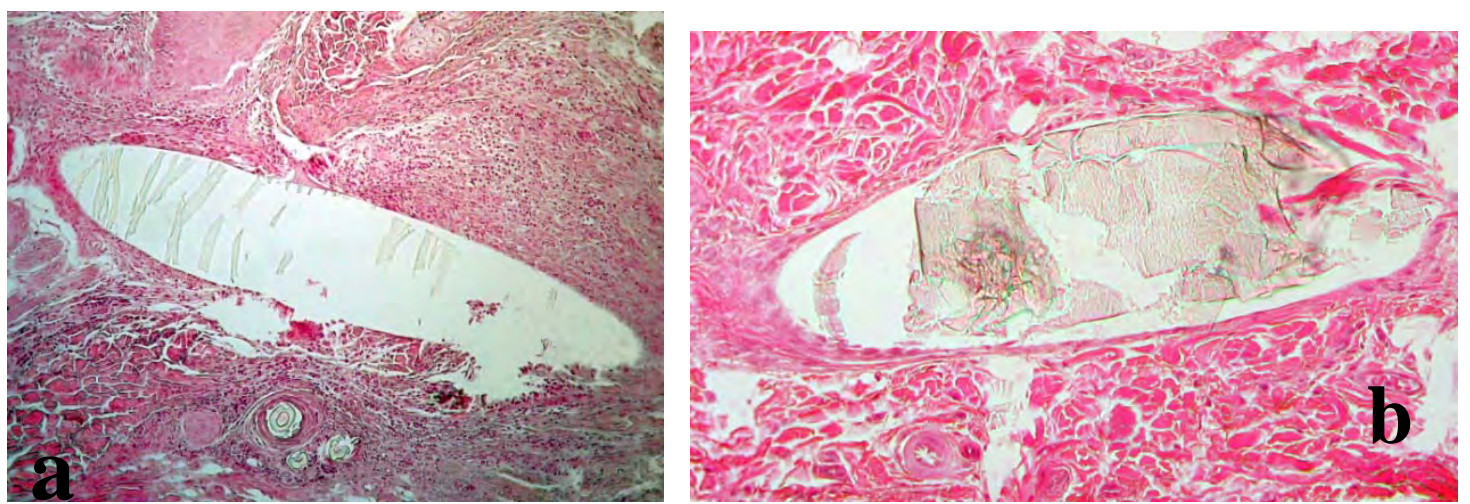

Figure 69a,b. Minimal to medium tissue reaction, composed of macrophages and fibrosis around suture material on the $28^{\text {th }}$ po.d.

The thickness of epidermis had decreased to $\mathrm{x}$ 1.2-2 times compared to the normal adjacent epidermis. In one animal (B7), one focal area with traumatic furunculosis was observed (Figure 70). 


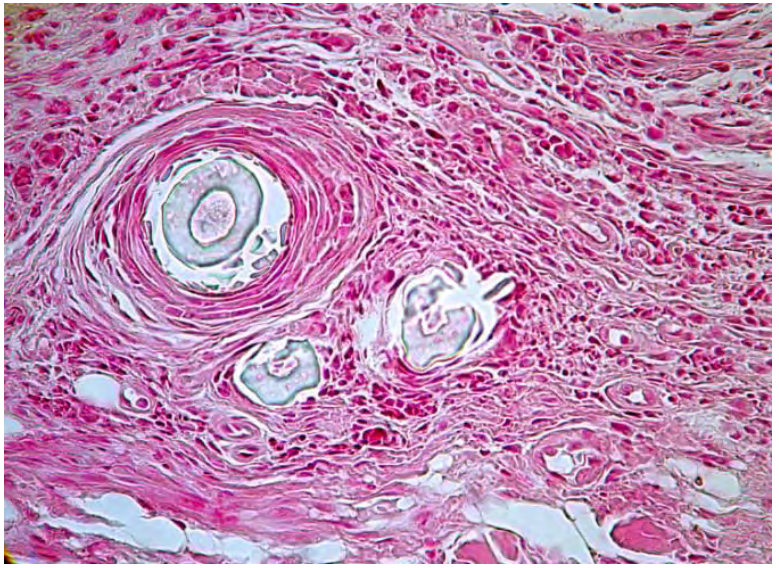

Figure 70. Focal area with traumatic furunculosis around hair particles inside the wound area on the 28th po.d.

In one animal (B9), diffuse dermatitis with lymphocytes, macrophages, and neutrophils was observed (Figure 71).
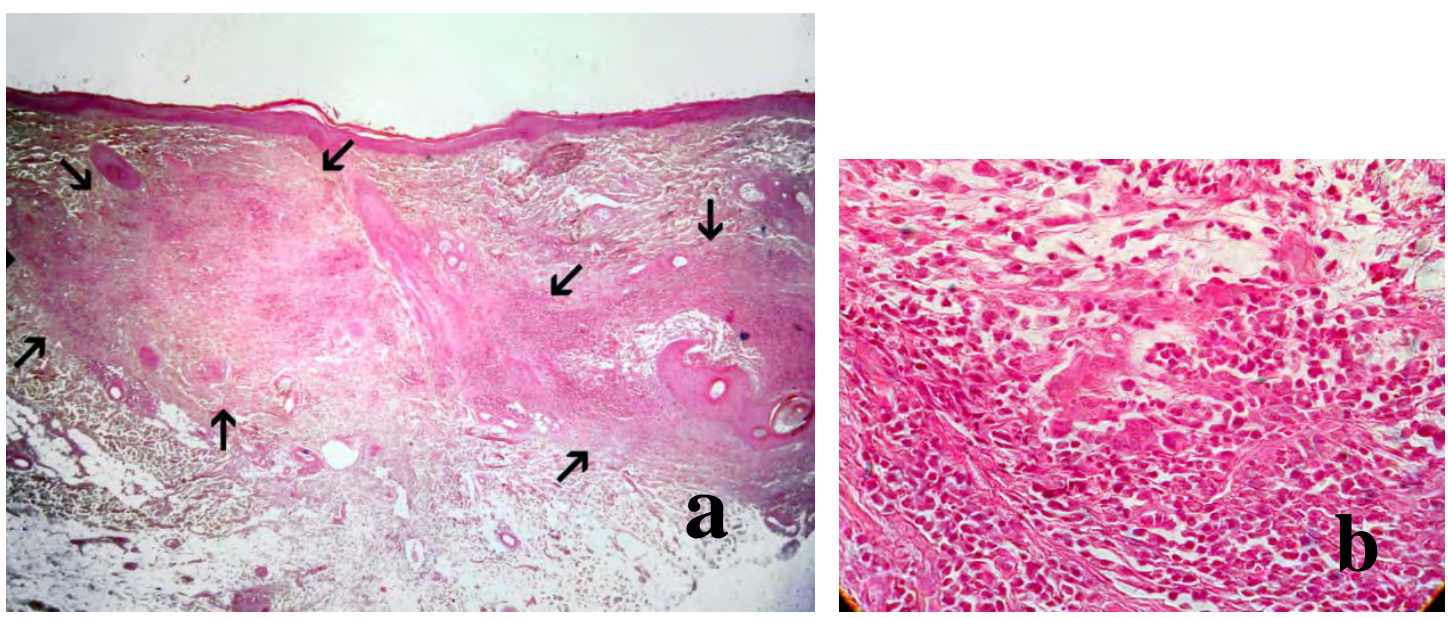

Figure 71a,b. Diffuse dermatitis (between arrows) with lymphocytes, macrophages, and neutrophils (b) at wound area on the $28^{\text {th }}$ po.d.

On the $180^{\text {th }}$ po.d., the main finding was the absence of glands and hair follicles from the wound area at the site of the incision, and the presence of thinner than normal collagen fiber bands (Figures 72a-d).
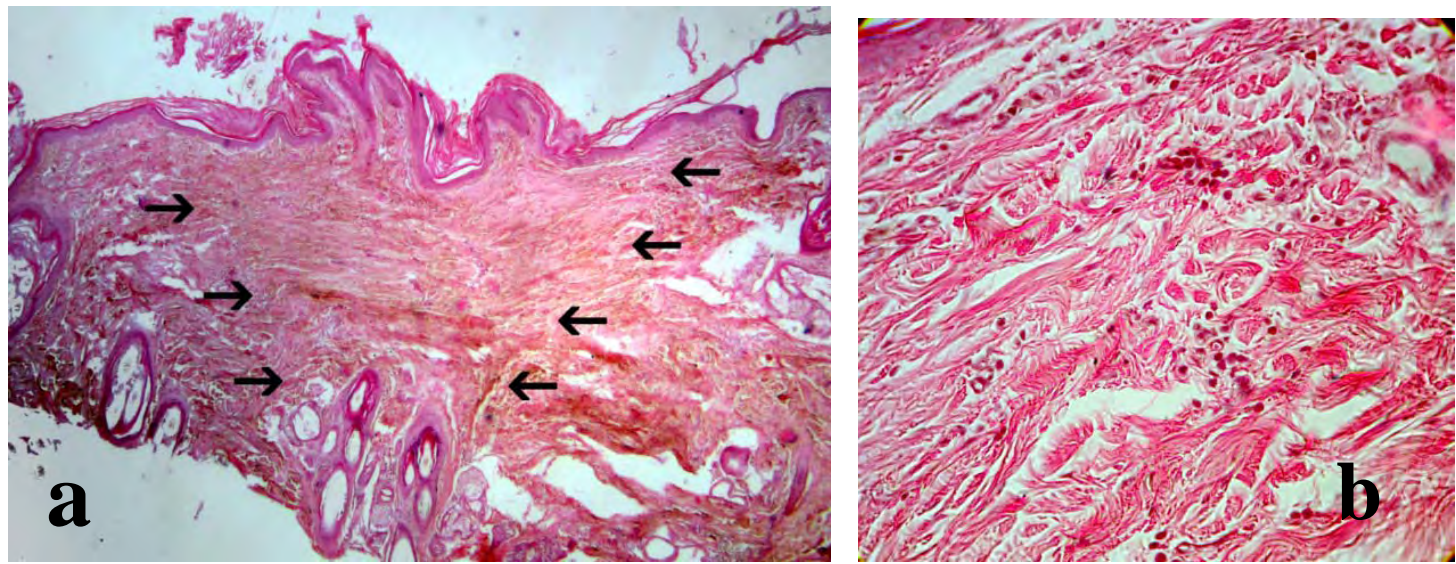

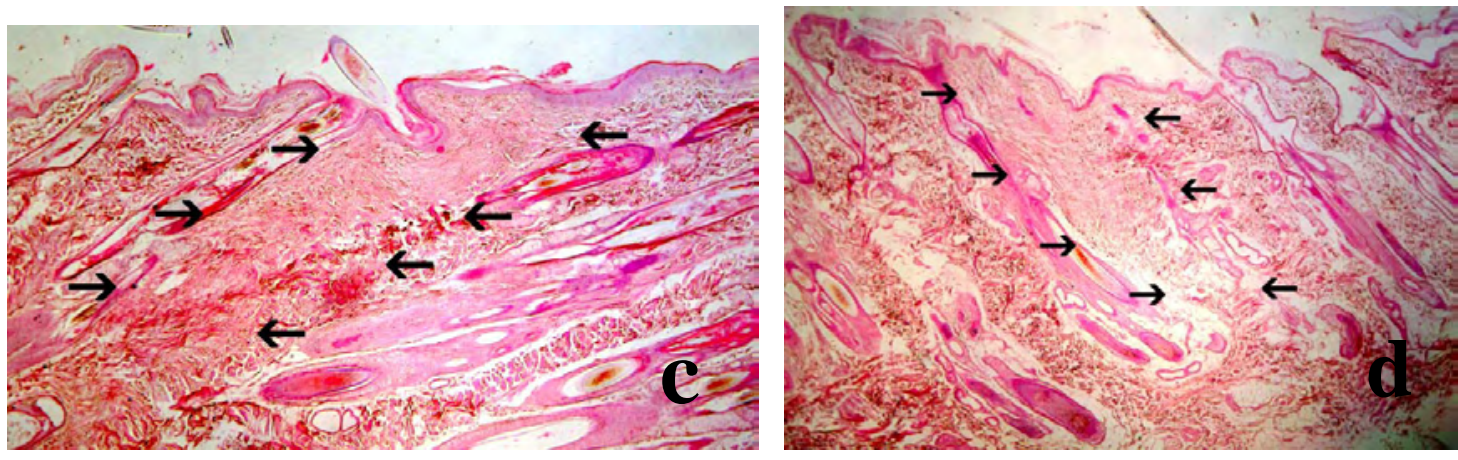

Figure 72a,b,c,d. Wound area at the site of incision (between arrows) on the $180^{\text {th }}$ po.d., without any glands or hair follicles, and with thinner than normal collagen fiber bands (on the left side of b).

No suture material was observed, due to its absorption. The thickness of the epidermis had decreased to $\mathrm{x}$ 1-1.5 times compared to the normal adjacent epidermis. In one animal (B8), a focal area with traumatic furunculosis was observed (Figure 73a,b).
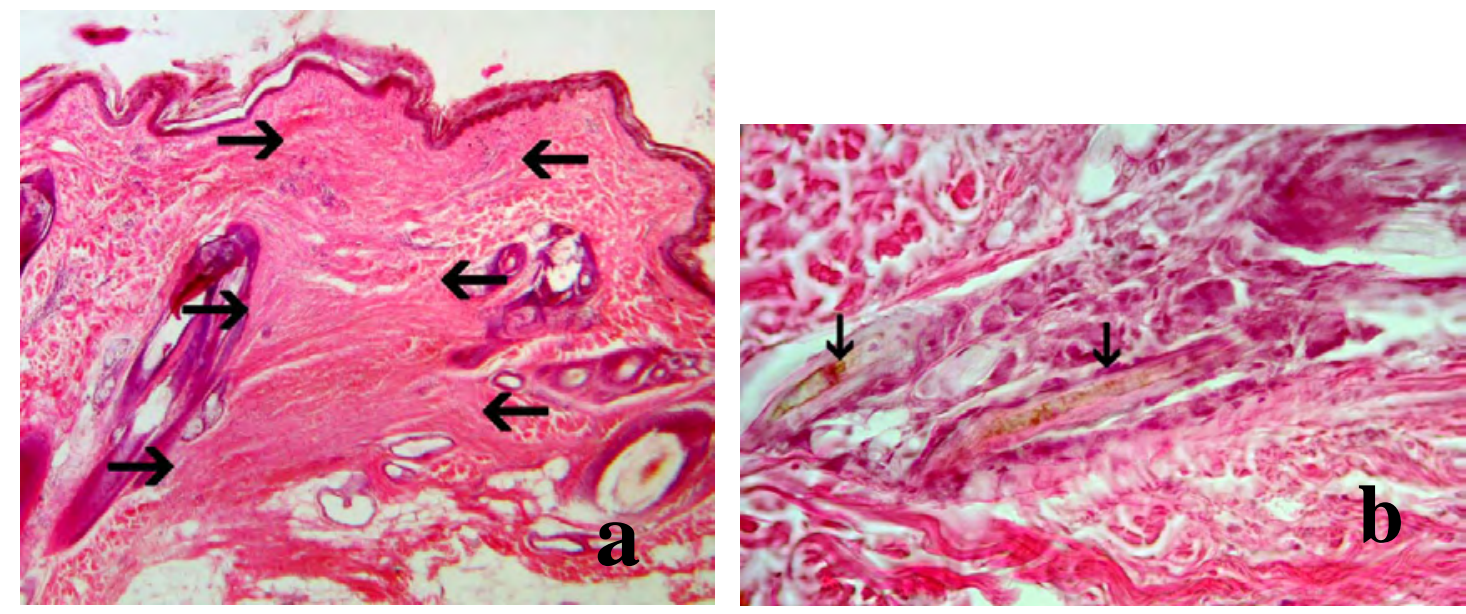

Figure 73a,b. Wound area at the site of incision (between arrows) on the $180^{\text {th }}$ po.d., without any glands or hair follicles, with thinner than normal collagen fiber bands and with hair fragments (arrows in b) initiating inflammatory reaction.

In one animal (B10), scattered macrophages and mast cells inside the dermis and the presence of a comedo were observed.

On the $365^{\text {th }}$ po.d., the main finding was the absence of glands and hair follicles from the wound area at the site of the incision, and the presence of thinner than normal collagen fiber bands. The thickness of the epidermis was increased x 1-1.5 times compared to the normal adjacent epidermis (Figure 74a,b). 

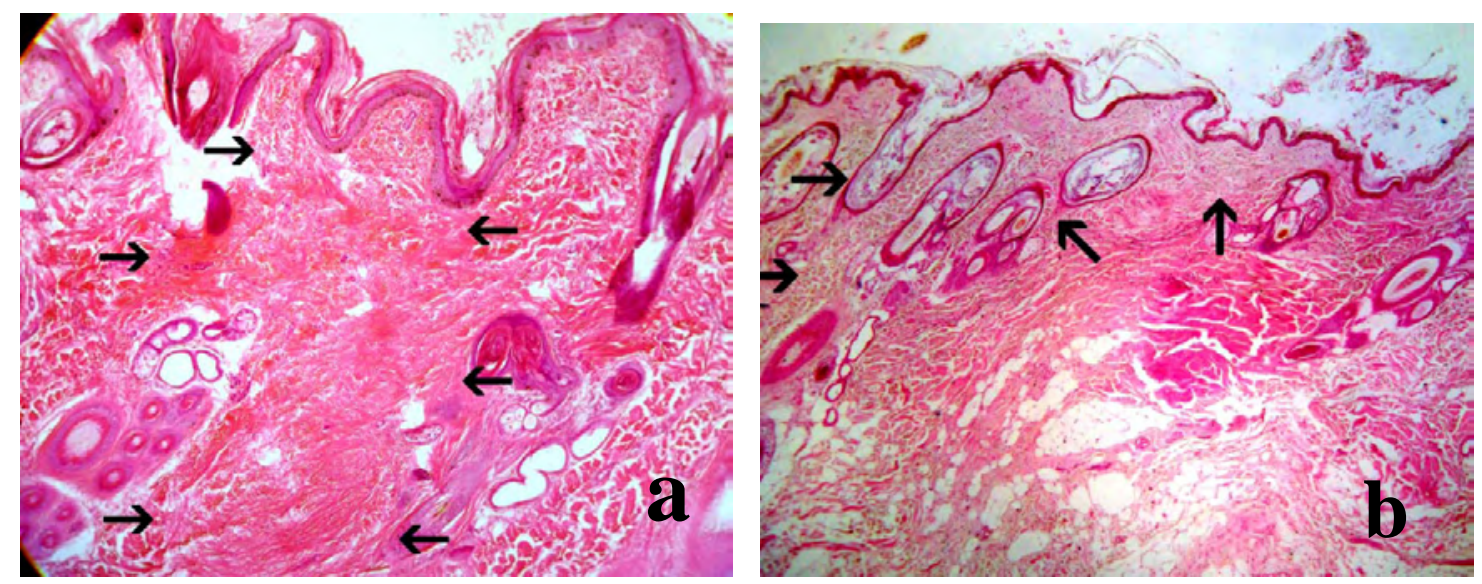

Figure 74a,b. Wound area at the site of the incision (between arrows) on the $365^{\text {th }}$ po.d., without any glands or hair follicles, and with thinner than normal collagen fiber bands. 


\section{Intradermal suture pattern with clips}

On the $7^{\text {th }}$ po.d. , the main finding was the infiltration of the wound area at the site of the incision by a small number of neutrophils, macrophages, and fibroblasts (Figure 75a-d).
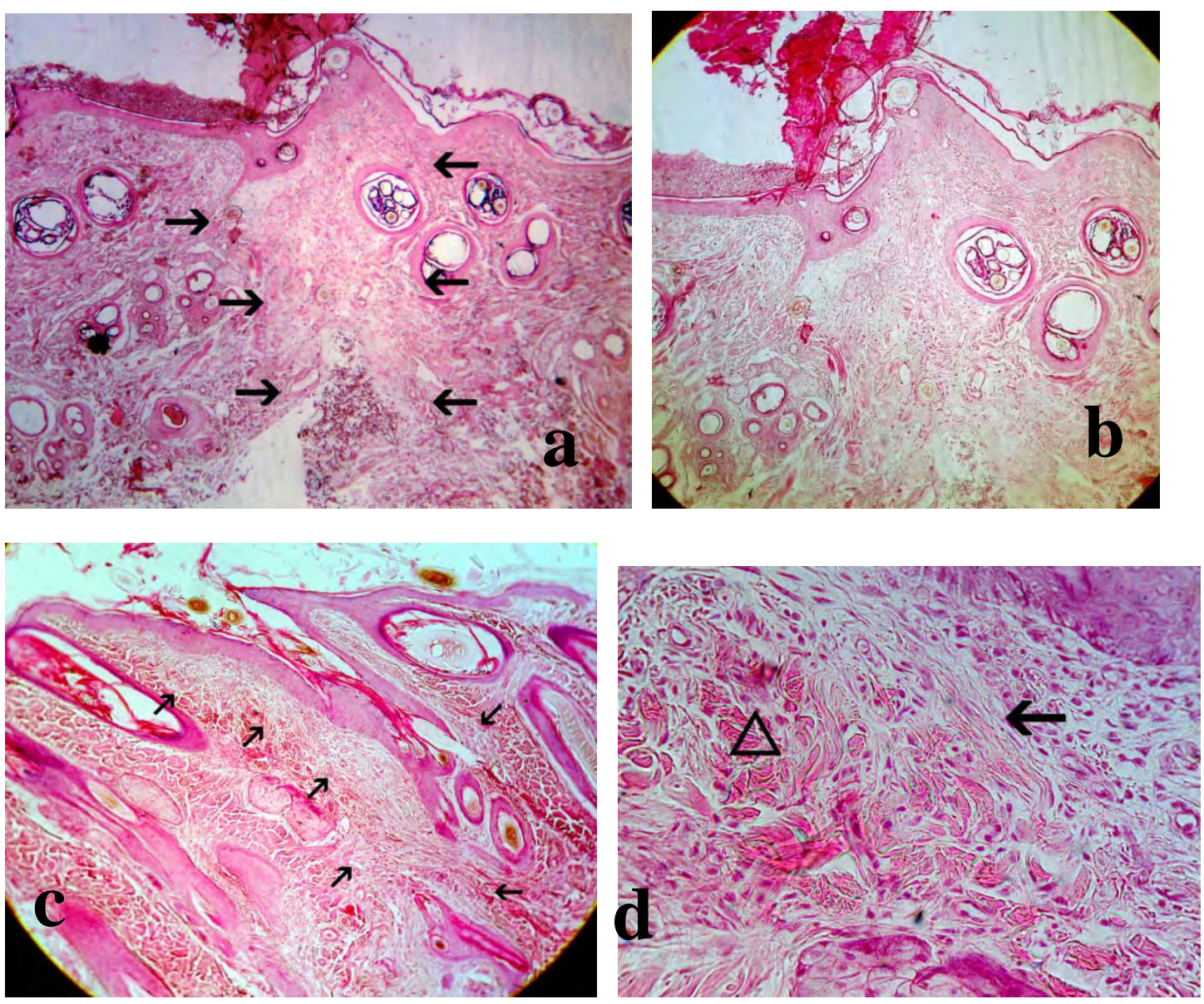

Figure 75a,b,c,d. Wound area at the site of incision (black arrows) with infiltration by a small number of neutrophils, macrophages, and fibroblasts on the $7^{\text {th }}$ po.d. d. Thinner than normal collagen fibers are present in the wound area (triangle: normal collagen fibers, arrow: newly formed collagen fibers)

Minimal tissue reaction, composed of macrophages and fibroblasts, was usually observed around the suture material (Figures 75, 76a,b).

The thickness of the epidermis was increased x1-2.5 times compared to the normal adjacent epidermis, whilst in one case increased x 3.5 times. The upper dermis next to the wound area was infiltrated by small numbers of macrophages and few mast cells (Figure 77). 


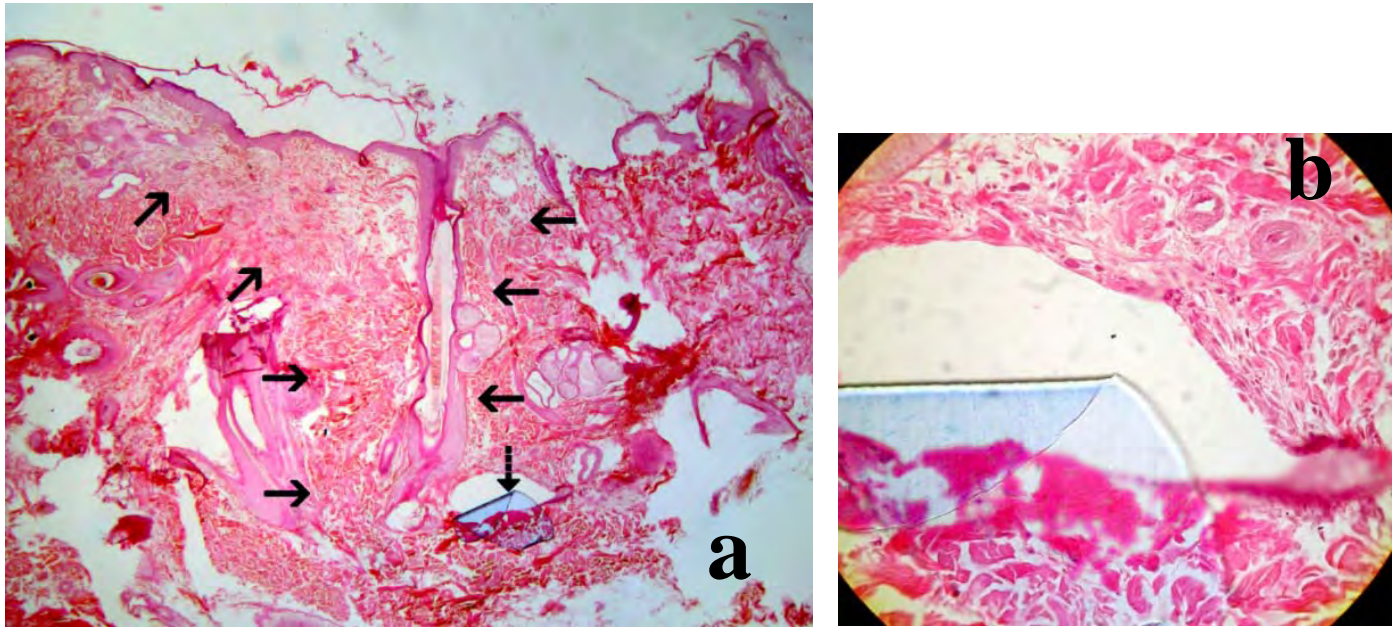

Figure 76a,b. Wound area at the site of incision (black arrows) on the $7^{\text {th }}$ po.d. b. Minimal tissue reaction composed of macrophages and fibroblasts around suture material (blue film partially relocated).

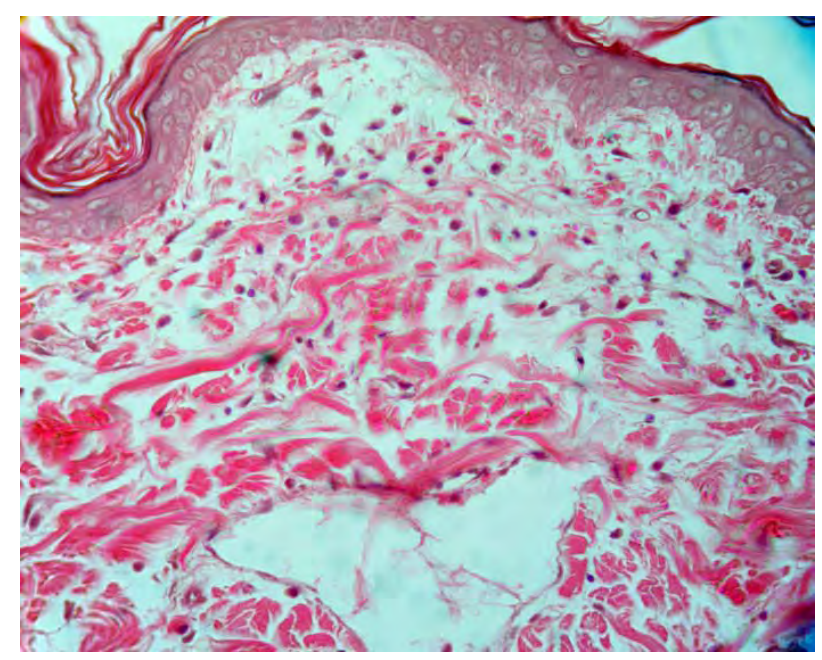

Figure 77. Upper dermis next to the wound area infiltrated by a small number of macrophages and few mast cells.

On the $14^{\text {th }}$ po.d. , the main finding was infiltration of the wound area at the site of the incision by fibroblasts and a small number of macrophages. The thickness of the epidermis was increased x1.5-3.5 times compared to the normal epidermis (Figure 78a,b). 

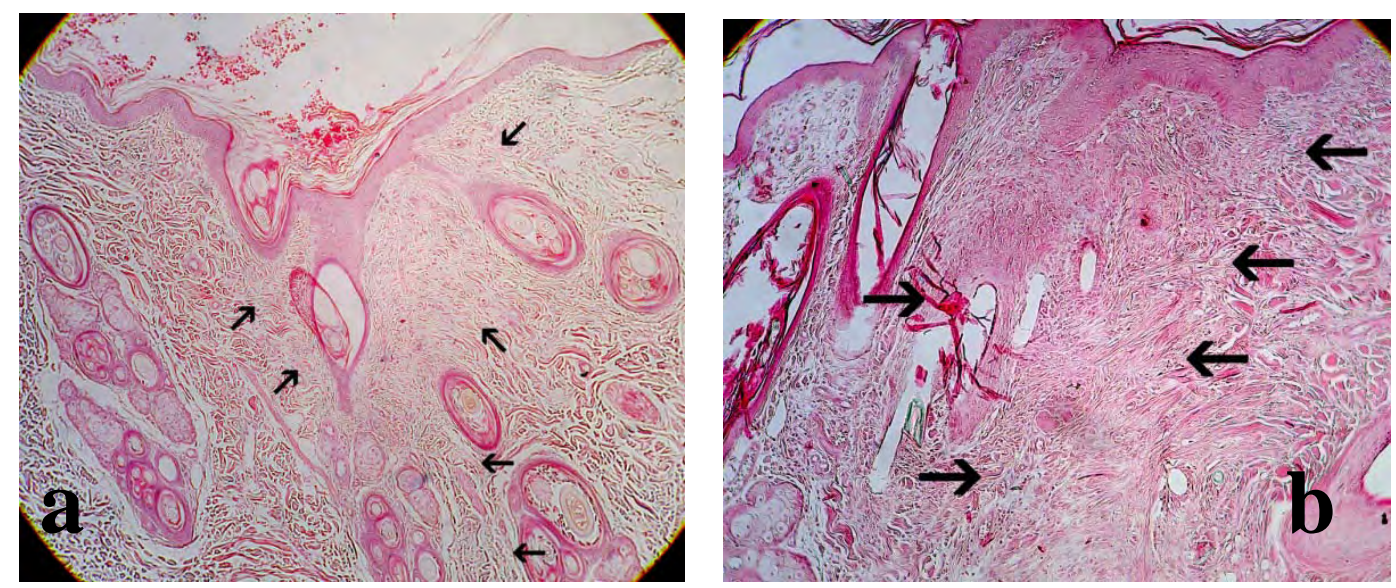

Figure 78a,b. Wound area at the site of the incision (black arrows) on the $14^{\text {th }}$ po.d., with infiltration by fibroblasts and a small number of macrophages.

The upper dermis next to the wound area was infiltrated by few macrophages and mast cells. In one animal (B7), a nodular accumulation of macrophages, neutrophils, and lymphocytes in the subcutaneous tissue was observed (Figure 79). Three foci with traumatic furunculosis were found (Figure 80). Some remnants of suture material were found in one sample (Figure 81).
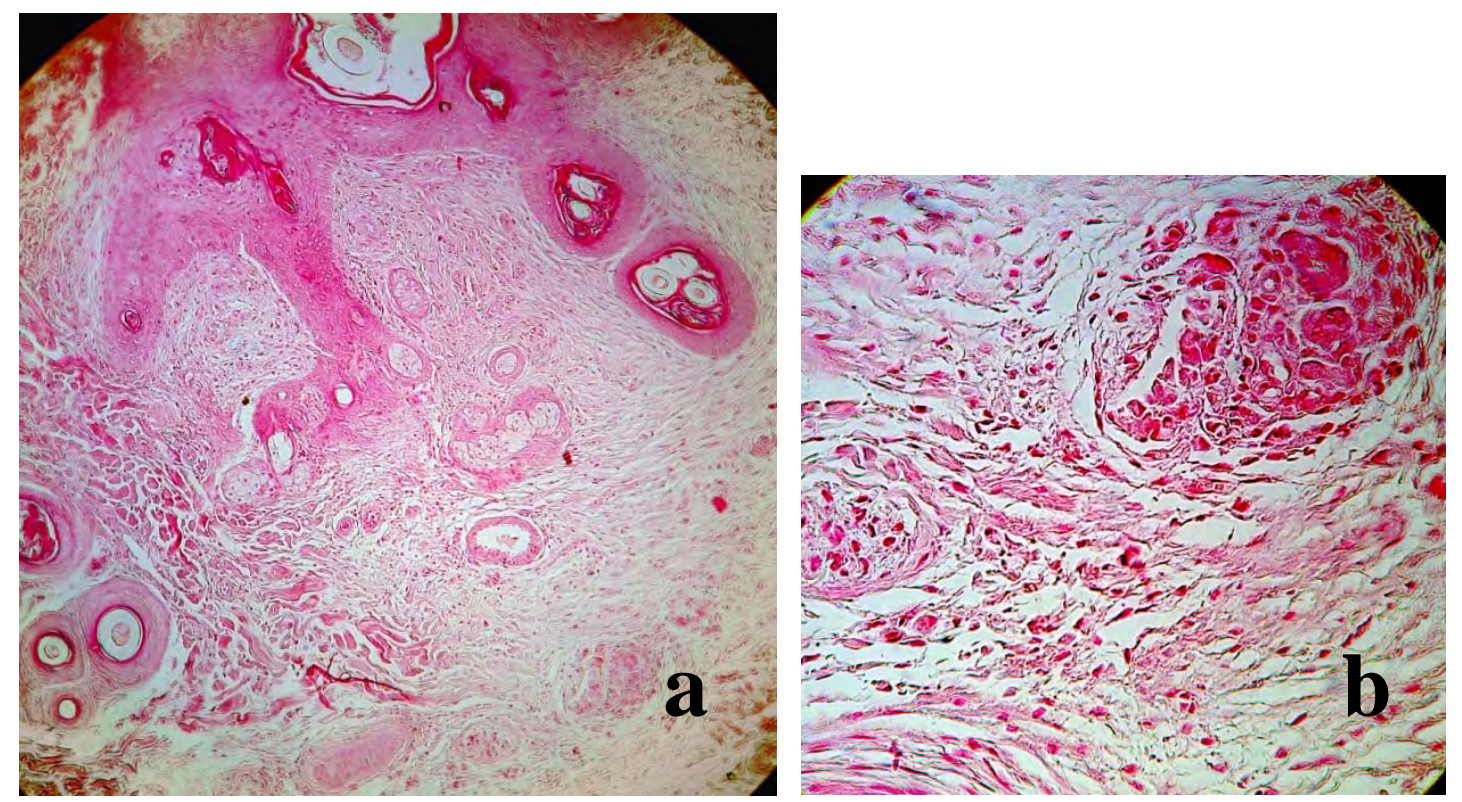

Figure 79a,b. Wound area at the site of the incision on the $14^{\text {th }}$ po.d with a nodular accumulation of macrophages, neutrophils, and lymphocytes at the subcutaneous tissue (b). 

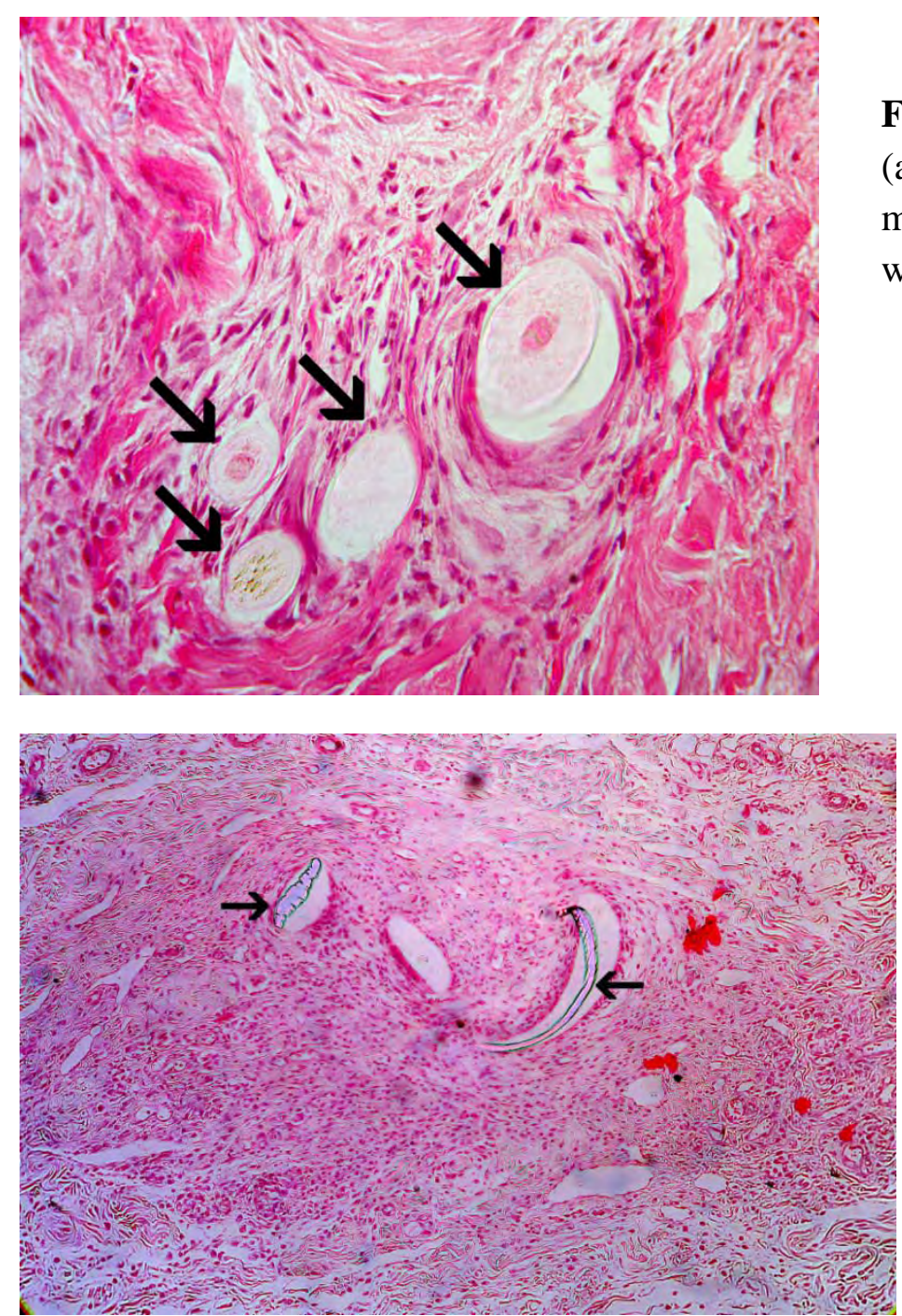

Figure 80. Hair fragments (arrows) initiating inflammatory reaction at the wound area.

Figure 81. Remnants of suture material (arrows) at the wound area after suture removal.

On the $\mathbf{2 8}^{\text {th }}$ po.d., the main finding was the absence of glands or hair follicles from the wound area at the site of the incision, where thinner than normal bands of collagen, and minimal to medium number of macrophages were observed (Figure 82). The thickness of the epidermis was decreased $x$ 1-1.5 times compared to the normal adjacent epidermis, whilst in one case it was x 2.5 times the normal epidermis. In the animal B8, an area with traumatic furunculosis was observed (Figure 83a,b). 

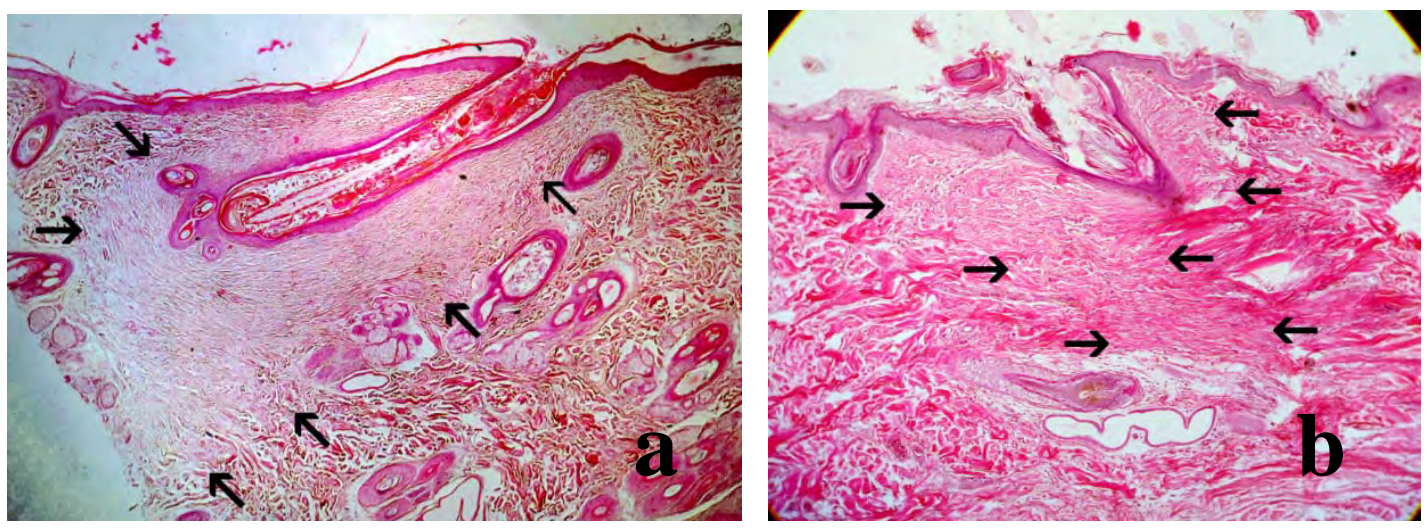

Figure 82a,b. Wound area at the site of the incision (black arrows), without any glands or hair follicles, with thinner than normal bands of collagen, and minimal to medium number of macrophages, on the $28^{\text {th }}$ po.d.
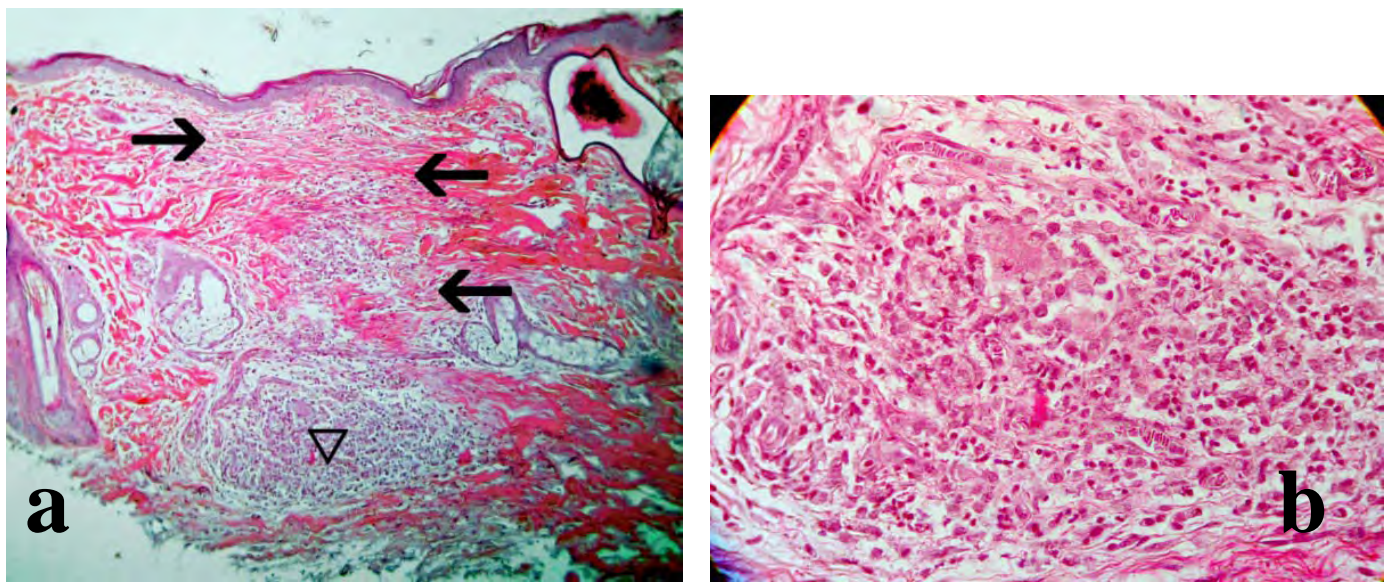

Figure 83a,b. Wound area at the site of the incision (black arrows) on the $28^{\text {th }}$ po.d., without any glands or hair follicles, with thinner than normal bands of collagen, and with an area with traumatic furunculosis (a triangle, b).

On the $\mathbf{1 8 0}^{\text {th }}$ po.d. , the main finding was the absence of glands or hair follicles from the wound area at the site of the incision, where thinner than normal bands of collagen were observed (Figure 84). The thickness of the epidermis was x 1-1.5 times compared to the normal adjacent epidermis. In one animal (B9), hyperplasic epidermis with dermal proliferation and a comedo were observed (Figure 85).

On the $\mathbf{3 6 5}^{\text {th }}$ po.d., the main finding was the absence of glands or hair follicles from the wound area at the site of the incision, where thinner than normal collagen fiber bands were observed. The thickness of the epidermis was x1-1.5 times compared to the normal adjacent epidermis (Figure 86a,b). 

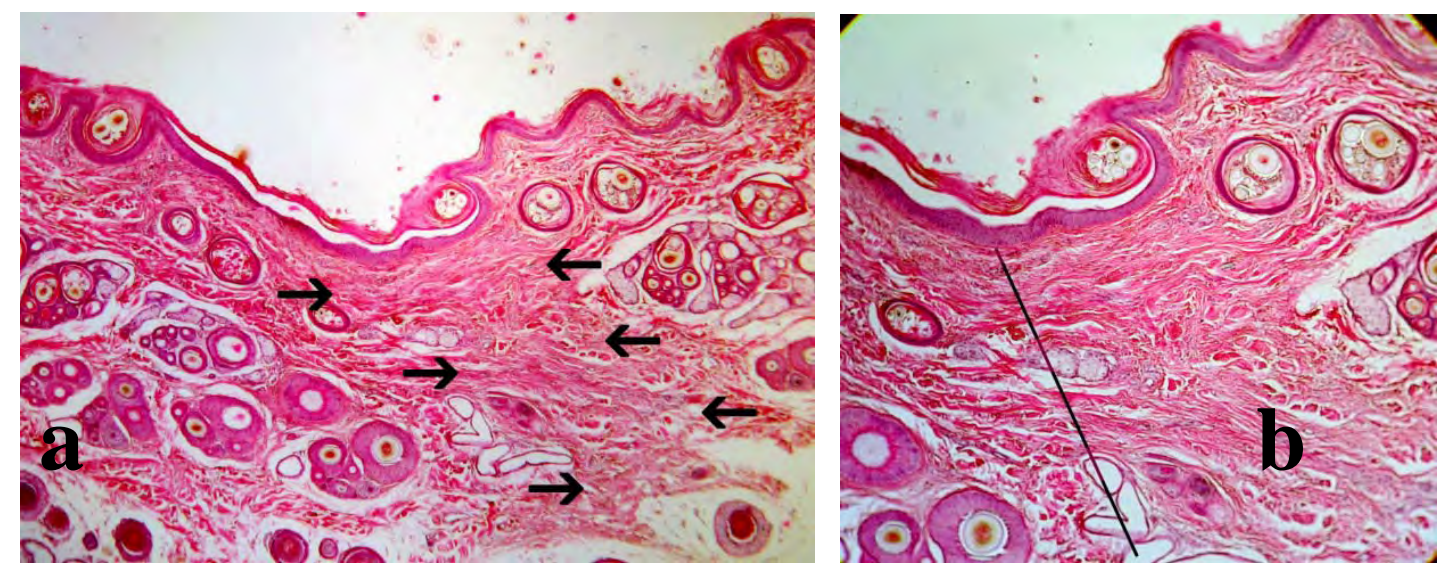

Figure 84a,b. Wound area at the site of the incision (black arrows) on the $180^{\text {th }}$ po.d., without any glands or hair follicles and with thinner than normal bands of collagen (b, on the right side of the black line).

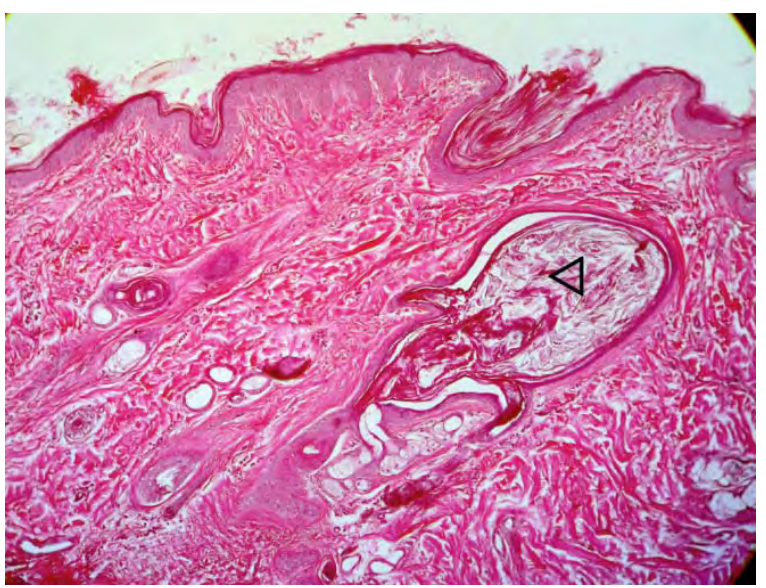

Figure 85. Wound area at the site of the incision on the $180^{\text {th }}$ po.d., showing hyperplasic epidermis with dermal proliferation and a comedo (triangle).
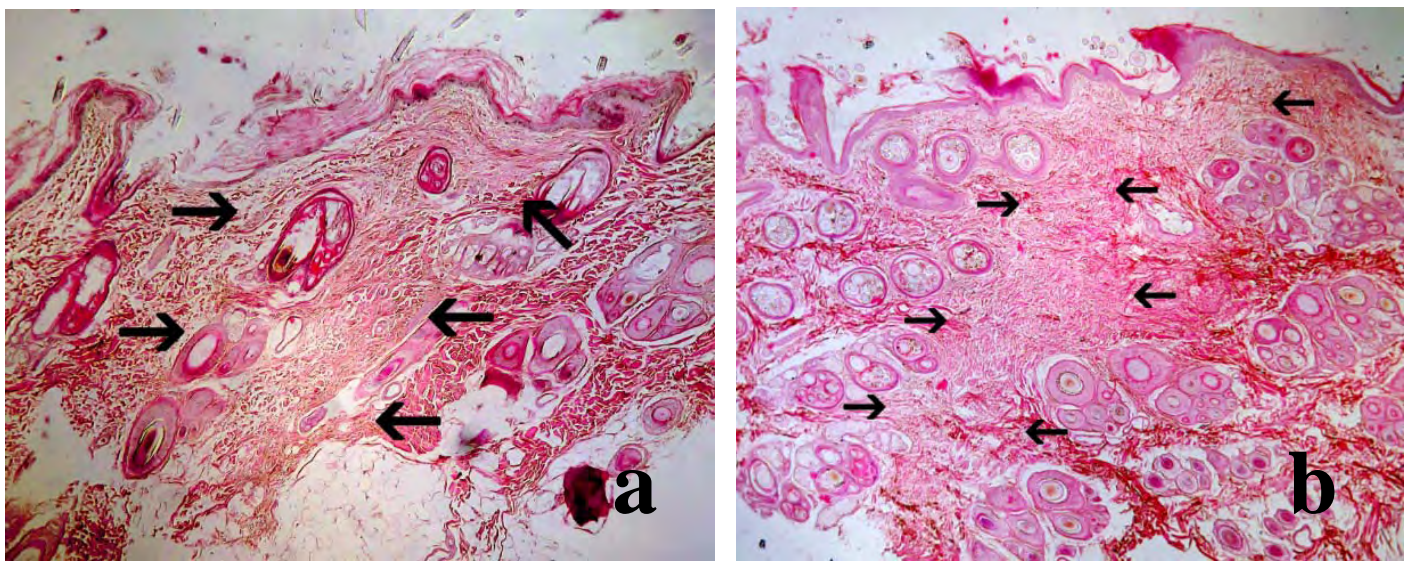

Figure 86a,b. Wound area at the site of the incision (black arrows) on the $365^{\text {th }}$ po.d., without any glands or hair follicles and with thinner than normal bands of collagen. 


\section{Total evaluation of each technique on the $7^{\text {th }}, 14^{\text {th }}, 28^{\text {th }}, 180^{\text {th }}$ and $365^{\text {th }}$ po.d.}

On the $\underline{7}^{\text {th }}$ po.d., the median total evaluation score was $18.6(17.5-21.5)$ as regards staples, 20.1 (19-22.2) as regards glue, 17.2 (16.5-18.5) as regards intradermal B and 17.7 (17-18.7) as regards intradermal C. Glue differed significantly from intradermal $\mathrm{B}(\mathrm{P}=0.007)$.

On the $14^{\text {th }}$ po.d., the median total evaluation score was 16.7 (14-20.7) as regards staples, 16.6 (14.2-18.2) as regards glue, 12.5 (12.2-15) as regards intradermal B and $13.5(12.5-14)$ as regards intradermal C.

On the $28^{\text {th }}$ po.d. the median total evaluation score was $11.7(8.2-14.7)$ as regards staples, 13.5 (9-15) as regards glue, 9.5 (8.5-10.2) as regards intradermal B and 8 (7.510) as regards intradermal $C$.

On the $180^{\text {th }}$ po.d. the median total evaluation score was 7.8 (6-9) as regards staples, 9.1 (6-10) as regards glue, 4.1 (3-6.7) as regards intradermal B and 4.7 (2-6) as regards intradermal $\mathrm{C}$.

On the $365^{\text {th }}$ po.d. the median total evaluation score was 4 (3-5.5.) as regards staples, 6.5 (4-9.2) as regards glue, 2.1 (1.2-6) as regards intradermal B and 3.5 (2.76.7) as regards intradermal $\mathrm{C}$.

On the $14^{\text {th }}, 28^{\text {th }}, 180^{\text {th }}$ and $365^{\text {th }}$ po.d., the comparisons between techniques revealed no significant differences ( $\mathrm{P}$ values are presented in Table 10). The median total evaluation score is presented in Figure 87. 
Table 10. $\mathrm{P}$ values of comparisons between techniques on the $7^{\text {th }}, 14^{\text {th }}, 28^{\text {th }}, 180^{\text {th }}$ and $365^{\text {th }}$ po.d.

\begin{tabular}{|c|c|c|c|c|}
\hline Technique & po.d. & glue & $\begin{array}{c}\text { intradermal } \\
\text { B }\end{array}$ & $\begin{array}{c}\text { intradermal } \\
\text { C C }\end{array}$ \\
\hline \multirow{4}{*}{ staples } & $7^{\text {th }}$ & 0.165 & 0.165 & 0.315 \\
\cline { 2 - 5 } & $14^{\text {th }}$ & 0.853 & 0.043 & 0.052 \\
\cline { 2 - 5 } & $28^{\text {th }}$ & 0.436 & 0.436 & 0.113 \\
\cline { 2 - 5 } & $180^{\text {th }}$ & 0.353 & 0.143 & 0.043 \\
\cline { 2 - 5 } glue & $365^{\text {th }}$ & 0.156 & 0.549 & 0.780 \\
\hline \multirow{5}{*}{ intradermal B } & $7^{\text {th }}$ & & $\mathbf{0 . 0 0 7}$ & 0.023 \\
\cline { 2 - 5 } & $14^{\text {th }}$ & & 0.075 & 0.089 \\
\cline { 2 - 5 } & $28^{\text {th }}$ & & 0.190 & 0.024 \\
\cline { 2 - 5 } & $180^{\text {th }}$ & & 0.143 & 0.043 \\
\cline { 2 - 5 } & $365^{\text {th }}$ & & 0.075 & 0.123 \\
\cline { 2 - 5 } & $7^{\text {th }}$ & & & 0.353 \\
\cline { 2 - 5 } & $14^{\text {th }}$ & & & 0.631 \\
\cline { 2 - 5 } & $28^{\text {th }}$ & & & 0.489 \\
\cline { 2 - 5 } & $180^{\text {th }}$ & & & 0.796 \\
\hline $365^{\text {th }}$ & & & 0.481 \\
\hline
\end{tabular}

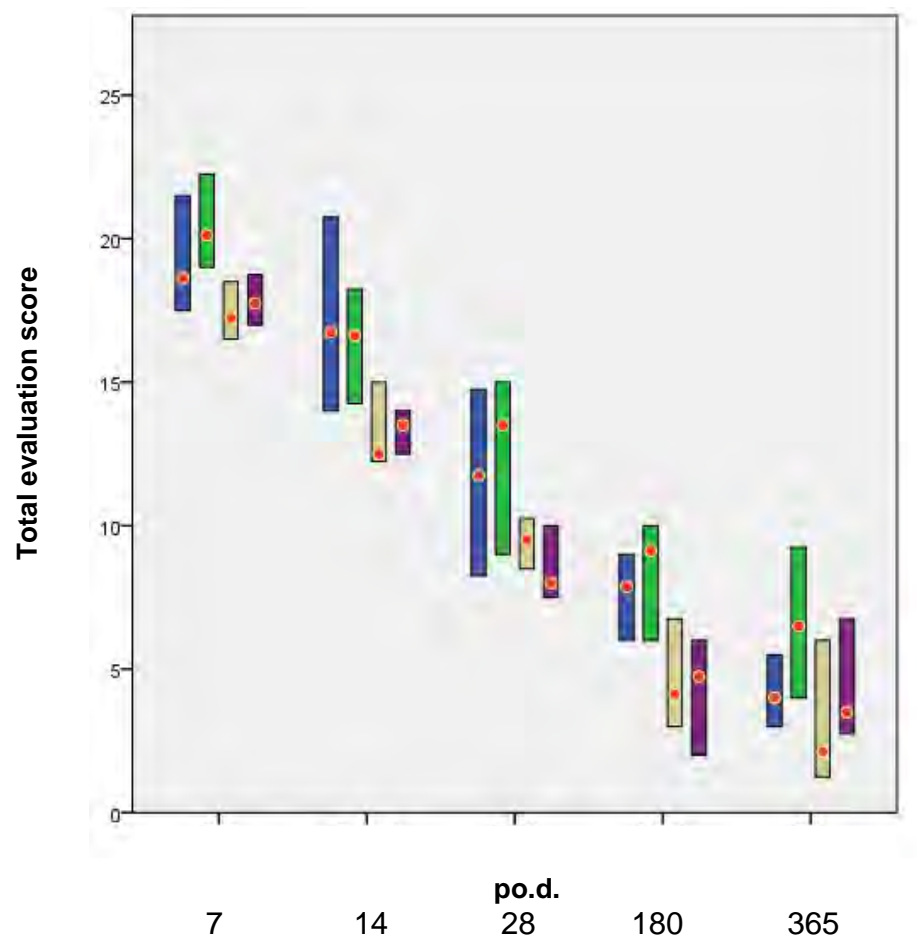

Figure 87. Median (red dot) and interquartile range (column) of total evaluation score for each technique (blue: staples, green: glue, gray: intradermal $\mathrm{B}$ and purple: intradermal C) on the $7^{\text {th }}, 14^{\text {th }}, 28^{\text {th }}, 180^{\text {th }}$ and $365^{\text {th }}$ po.d. 


\section{Correlations}

Correlation between $\mathbf{u} / \mathrm{s}$ estimated wound area and histologically estimated inflammation

To examine the association between the $\mathrm{u} / \mathrm{s}$ estimated wound area at the biopsy site and the histologically estimated inflammatory reaction at the same area, the Spearman correlation test was used. The test showed that there is a statistically significant positive correlation between the two parameters $(\mathrm{r}=0.699, \mathrm{P}=0.001)$, i.e. the larger the $\mathrm{u} / \mathrm{s}$ estimated wound area, the more intense the inflammatory reaction in the same area.

Correlation between $\mathrm{u} / \mathrm{s}$ estimated wound area and histologically estimated scar width

Spearman's rho for the $\mathrm{u} / \mathrm{s}$ estimated wound area at the biopsy site and the histologically estimated scar width at the same area showed that there is a statistically significant positive correlation between the two parameters $(r=0.342, p<0.001)$, i.e. the larger the $\mathrm{u} / \mathrm{s}$ estimated wound area, the largest the scar width.

\section{Correlation between $\mathbf{u} / \mathbf{s}$ estimated wound area and clinically evaluated skin} thickening

Spearman's correlation was implemented to test the association between the $\mathrm{u} / \mathrm{s}$ estimated wound area and the clinically evaluated skin thickening. The results showed that there is a statistically significant positive correlation between the two parameters $(r=0.682, p<0.001)$, i.e. the larger the $\mathrm{u} / \mathrm{s}$ estimated wound area, the larger the skin thickening.

\section{Correlation between $\mathrm{u} / \mathrm{s}$ estimated wound area and clinically evaluated scar width}

In order to find out if there is any correlation between the $\mathrm{u} / \mathrm{s}$ estimated wound area and the clinically evaluated scar width, the Spearman correlation test was used. The test showed that there is a statistically significant positive correlation between the two parameters $(r=0.298, \mathrm{p}<0.001)$, i.e. the larger the $\mathrm{u} / \mathrm{s}$ estimated wound area, the larger the scar width. 
Institutional Repository - Library \& Information Centre - University of Thessaly 26/04/2023 13:56:30 EEST - 54.147.182.235 


\section{DISCUSSION}

Up to now, the assessment of wound healing is mainly based on clinical evaluation of the wound, on the frequency of wound complications and on the cosmetic appearance of the wound. Histological evaluation of wound healing is very important but is invasive. The ultrasound evaluation of wound healing is not invasive and so far it has been used for the assessment of open skin wounds that are left to close by second intention. The aim of the present study was to compare the efficacy of various methods used for the closure of skin incisions in the dog by using cosmetical, clinical, highresolution ultrasonography and histological evaluation. Furthermore, the ultrasonographic appearance of the healing skin was compared with clinical and histological evaluations.

\section{Surgical operation}

As was expected, the application of staples and tissue adhesive was easier in comparison to the intradermal suture patterns. Staples application required significantly less time than tissue glue application and intradermal suture materials implementation. Tissue glue application required more time compared to staples application and less compared to intradermal suture materials implementation, both differences being statistically significant. The successful tissue glue application as a thin and single layer over the apposed wound edges only required the proper eversion of wound edges and the flow control of the adhesive for avoiding deposition into the wound area. However, in two animals small quantities of glue substance were found inside wound area, indicating that proper care should be taken during application, even if this increases the time needed. Among the intradermal suture patterns, the pattern with clips was easier to perform compared to the pattern with burying of the knot and required significantly less time than the second. Tying and correct burying of the knots into the subcutaneous tissue was more technically demanding and timeconsuming than the stabilization of suture material with clips, but not a reason for an experienced surgeon to avoid this technique.

Although the time required for the intradermal suture materials implementation in a $12 \mathrm{~cm}$ incision is significantly longer in comparison to the other two techniques, this time is not considered long in relation to the overall time of a surgical operation and should not be a criterion for not using an intradermal suture pattern. However, when the time of skin wound closure is an important factor in the emergency setting, in nonstabilized patients, or when the length of the skin to be sutured is considerable, stapling should be the first choice.

Chow et al (2010), who performed a systematic review and meta-analysis to compare the performance of tissue glue with standard wound-closure methods used on skin incisions in the elective surgical setting, found that time for skin wound closure by the majority of studies was considerably faster with glue compared to sutures. They also claimed that overall, in the elective surgical setting, the relative time taken for skin wound closure might be negligible, regardless of which method is used, as 
skin wound closure occurs at the end of a surgical procedure, which will normally take a great deal of time longer than the time needed to close the skin wound (Chow et al 2010).

In relation to the suture material, handling the 4-0 poliglecaprone 25 is easier than handling the 4-0 polypropylene, as the first one is more pliable.

It should also be mentioned that staples removal by utilizing the special tool was easy. The removal of 4-0 polypropylene was also easy and without resistance, by applying gentle traction to one end of the suture material. The n-butyl tissue adhesive was degraded in all incisions without applying acetone.

\section{Cosmetic evaluation of the incisions}

Although there was a slight difference between the blind assessor's evaluation, there was no statistical difference between their scores. In general, the scar appearance for all techniques used was cosmetically evaluated by the assessors with a good rating. In period A $\left(1^{\text {st }}-8^{\text {th }}\right.$ po.d.), the second assessor's rating was statistically more stringent than the first's. In that time period, the appearance of the incisions as regards staple, intradermal $\mathrm{B}$, and intradermal $\mathrm{C}$ was cosmetically evaluated as better than that of glue, however without a statistically significant difference. The intense erythema and skin thickening observed with incisions closed with tissue adhesive may have contributed to that rating. In period B $\left(9^{\text {th }}-21^{\text {st }}\right.$ po.d.) the appearance of the wound area as regards staples and intradermal $\mathrm{C}$ was evaluated as cosmetically better than glue, as closing materials had been removed for these techniques. In period $C\left(22^{\text {nd }}-63^{\text {rd }}\right.$ po.d. $)$, no statistically significant difference between the assessors' ratings was revealed and the cosmetic appearance of the incisions was evaluated as being significantly better as regards intradermal $\mathrm{C}$ in comparison to glue or intradermal $\mathrm{B}$. In that period, wounds closed with tissue adhesive still showed erythema, skin thickening and wide scar, whilst in intradermal suture pattern with burying of the knots, mechanical irritation of the wound area by the suture material promotes inflammation. In period D $\left(64^{\text {th }}-180^{\text {th }}\right.$ po.d.) the appearance of the incisions for intradermal $B$ and intradermal $C$ was cosmetically evaluated as better than for staples and glue; however, a statistically significant difference was revealed only between intradermal $\mathrm{C}$ and glue. In period $\mathrm{E}$ $\left(181^{\text {th }}-365^{\text {th }}\right.$ po.d.), the appearance of the incisions according to the assessors' evaluation was best for intradermal B, followed by intradermal C, staples, and glue. Statistically significant differences were revealed between intradermal B, intradermal Cand staples in comparison to glue. Consequently, although the cosmetic evaluation of wound area was gradually improving for all techniques, there were significant differences between glue and the other techniques from the $9^{\text {th }}$ until $365^{\text {th }}$ po.d.

Obermair et al (2007) compared the cosmetic result of the wounds (based on surgeons assessment) between the staples application and the intradermal techniques with burying the knots using poliglecaprone 25 and poliglecaprone 6211 suture materials in gynaecological abdominal surgery. The outcome by using staples application was evaluated worst at 1 week and 3 months after surgery compared to the other two groups, whereas there was no difference in surgeons' assessment at 6 weeks after surgery. Switzer et al (2003) compared the cosmetic outcome of wounds after 
four weeks in inguinal herniorrhaphy incisions using 2-octylcyanoacrylate tissue adhesive and 4-0 poliglecaprone 25 in a running subcuticular closure. No significant difference was observed between the techniques.

De Graaf et al (2012) compared the staples application with the intradermal suture pattern with burying the knots using 3/0 poliglecaprone 25 for wound closure after caesarean sections. They deduced that the cosmetic evaluation of the incisions on the $6^{\text {th }}$ month was similar between the two techniques. Livesey et al (2009) compared the cosmetic appearance of the scar in surgically closed long incisions, performed during total hip replacement, following the application of skin tissue adhesive (n-butyl and octyl-blend 70) and surgical staples. No significant difference was observed between the techniques at the 3-month evaluation. Also, Chibbaro and Tacconi (2009), who compared the cosmetic appearance of the scar in surgically closed long incisions, performed during brain surgery, following the application of skin adhesive (n-butyl and 2-octyl cyanoacrylates), surgical staples and traditional skin sutures (nylon), observed no significant difference between the techniques at the 3-month, 6-month and 12-month follow up.

Sylvestre et al (2002) compared the intradermal suture pattern with burying the knots using poliglecaprone 25 and the simple interrupted pattern using poliglecaprone or polypropylene for skin closure after canine ovariohysterectomy. They observed that at the $2^{\text {nd }}$ post day, the incision closed with intradermal pattern was cosmetically worse in relation to that closed with simple interrupted pattern. However, after 10-14 days, the incision closed with intradermal pattern was cosmetically better.

Cromi et al (2010), while closing incisions following cesarean section, observed similar cosmetic appearance of the scar 2 and 6 months postpartum, when compared (i) staples with subcuticular running suture with 3-0 monofilament glycolide, $\varepsilon$ caprolactone, and trimethylene-carbonate, with (ii) nonabsorbable monofilament suture made of polyamide polymers, or with (iii) a short-term synthetic absorbable braided and coated suture made of low-molecular-weight polyglycolic acid.

Gouletsou (2014), when compared the cosmetic outcome after suturing skin incisions with simple interrupted suture pattern or intradermal suture pattern with burying of the knots using two different suture materials (poliglecaprone 25 or polyglitone 6211) and two sutures of different diameters (3-0 and 4-0), observed that the continuous intradermal suture pattern with burying of the knots performed with a 4/0 poliglecaprone 25 suture presented a better cosmetic outcome, although without a statistically significant difference.

\section{Clinical evaluation}

Wound repair by first intention was achieved normally with all techniques, without severe complications. Oedema, erythema, scar width, abscessation, hyperpigmentation, cross scaring, wound dehiscence and staples loss were the parameters assessed for the total clinical evaluation. 


\section{Skin thickening at the wound area}

Skin thickening was observed from the first po.d. in all techniques. The larger skin thickening was observed between $3^{\text {rd }}$ or $4^{\text {th }}$ po.d. as regards staples and at the $1^{\text {st }}$ po.d. as regards glue, intradermal B' and intradermal C. Afterwards, skin thickening was decreased to a different degree for each technique until the $63^{\text {rd }}$ po.d.

In period A $\left(1^{\text {st }}-8^{\text {th }}\right.$ po.d. $)$, skin thickening was larger as regards staples in comparison to glue, intradermal $\mathrm{B}$, and intradermal $\mathrm{C}$; however statistically significant difference was observed only between staples in comparison to intradermal B and intradermal C. At the incisions closed with staples, the median increase of the skin thickness at the wound area was $2.5 \mathrm{~mm}$ (maximum $4.3 \mathrm{~mm}$ ). It should be taken under consideration that the staples must not be placed in close contact with the skin, in order to avoid ischemia, necrosis, cross scaring formation and possibly the loss of staples due to oedema. At the incisions closed with tissue adhesive, the median increase of skin thickness was $1.7 \mathrm{~mm}$ (maximum $2.6 \mathrm{~mm}$ ). Since there was no evidence of marked inflammation at the wound area due to incorrect application of the tissue adhesive, the postoperative skin thickening was the result of the technique, in which the tissue adhesive was joined only with the epidermis of the wound margins forming a flexible coating and not with the dermis of the wound margins. As the parts of the dermis of wound margins were simply in loose contact, the presented tissue swelling at the wound area was moderate. As regards the intradermal techniques, the skin thickening was smaller than with the previous. As both the dermis portions of the wound margins were held tightly throughout the whole length of the incision due to the suture material presence, the presented tissue swelling at the wound area was mild.

In_period B $\left(9^{\text {th }}-21^{\text {st }}\right.$ po.d.), as wound healing processed without complications, skin thickening was decreased for all techniques, without any statistically significant differences observed between them. Skin thickening was larger as regards staples and intradermal B in comparison to glue and intradermal C, while it was even smaller as regards intradermal $\mathrm{C}$. Almost from the beginning of this period $\left(10^{\text {th }}\right.$ day), only poliglecaprone 25 suture ( $3^{\text {rd }}$ technique) was still inside the wound area, since the staples and the polypropylene suture had been removed and the tissue adhesive had been degraded. Concerning the intradermal suture patterns, the larger skin thickening at the wound area was observed with intradermal $\mathrm{B}$, probably due to the presence of suture material into the wound at that period. It must be noted that with intradermal B, in the areas where the knots were buried, there was more pronounced swelling which remained longer than in the rest of the incision.

In period C $\left(22^{\text {nd }}-63^{\text {rd }}\right.$ po.d.), skin thickness at wound areas in all techniques was even more decreased, without significant differences between techniques. Skin thickness was larger with glue in comparison to the others, while it was the smallest with the $1^{\text {st }}$ and $4^{\text {th }}$ technique.

In periods D \& E, wound skin thickness decreased to pre-wounding skin thickness, whilst no significant differences were observed between the techniques.

In conclusion, until the $7^{\text {th }}$ po.d., skin thickening was present with all techniques but it was more pronounced when staples were placed. Afterwards, until the $21^{\text {st }}$ po.d., skin thickening declined with all techniques, with the incisions closed with staples 
having the larger decline, especially after staple removal on the $10^{\text {th }}$ po.d. Wound thickness declined even more after the $63^{\text {rd }}$ po.d., to return to almost normal afterwards.

Gouletsou (2014) observed that skin thickening at the wound area with the intradermal technique with burying the knots and using 4/0 absorbable monofilament suture materials was large on the $1^{\text {st }}$ po.d. and then it decreased gradually until the $20^{\text {th }}$ po.d. On the contrary, when intradermal suture pattern with burying of the knots was performed by using an absorbable monofilament suture material of a larger diameter $(3 / 0)$, the skin thickening was increasing from the $1^{\text {st }}$ until the $12^{\text {th }}$ po.d. and then it gradually decreased.

\section{Erythema presence}

Erythema was also observed in the wound area. From the $1^{\text {st }}$ until the $8^{\text {th }}$ po.d., it was more pronounced with staples in comparison to glue, intradermal $\mathrm{B}$, and intradermal C; however, no significant differences were observed between them. From the $9^{\text {th }}$ until the $21^{\text {st }}$ po.d., the extent of erythema decreased in all cases except one wound closed with staples, where the erythema insisted until the $42^{\text {nd }}$ po.d.

Although erythema is expected during the inflammation stage of the healing process, in this study erythema was mild with all techniques. Gouletsou (2014) had also observed erythema at wound areas in all incisions sutured with intradermal patterns, however, it was more intensive end lasted more, i.e. until the $49^{\text {th }}$ po.d. Gouletsou (2014) administered no antibiotics post surgery, as in the present study, and this may have caused a rather prolonged inflammation phase that aggravated erythema. It is also possible that in the present study, the removal of the closure materials performed on the $10^{\text {th }}$ po.d. might have contributed to the elimination of the erythema. However, the same outcome was also observed with intradermal B, although the suture remained in situ until absorption.

\section{Scar width}

An important clinical parameter of the skin healing process is the scar that forms on the skin at the wound area. On the first postoperative days, the scar was thin and covered with an eschar (scab). After the eschar had fallen off, the region of the scar was distinguished due to the different texture and because it was colorless and hairless.

In time period A ( $1^{\text {st }}-8^{\text {th }}$ po.d $)$, although the scar width was larger with staples and glue compared to intradermal $\mathrm{C}$, and even larger when compared to intradermal B, no statistically significant differences were observed between the techniques. The scar width was smaller with intradermal B and intradermal C, probably due to the stronger restraint of wound edges by the suture material present at the wound area.

In period $B\left(9^{\text {th }}-21^{\text {st }}\right.$ po.d. $)$, the scar width decreased with staples, remained small with intradermal B and intradermal $\mathrm{C}$, and increased considerably with glue. Statistically significant difference was observed between glue and intradermal B and intradermal C. The wider scar with glue could not be attributed to wound oedema, since this was moderate with that technique and did not differ from that observed with 
the other techniques. It is possible that with glue, the glue that attaches to the epidermal edges only, cannot apparently restrain dermis, so the scar is widening as healing proceeds.

In period C $\left(22^{\text {nd }}-63^{\text {rd }}\right.$ po.d.), the scar width was slightly decreased with the staples and intradermal $\mathrm{B}$ and it was considerably decreased with glue, probably due to wound contraction. However, it was slightly increased with intradermal C. Statistically significant difference was observed between glue and intradermal B only. The scar width was slightly increased with intradermal $C$ when compared to the previous time period, and to intradermal $\mathrm{B}$, probably due to the removal of suture material from the wound, which was holding the edges of the wound under dynamic contact when present.

In period D $\left(64^{\text {th }}-180^{\text {th }}\right.$ po.d. $)$, the scar width was slightly decreased with all techniques, however, no statistically significant differences were observed between techniques.

In period $E\left(181^{\text {st }}-365^{\text {th }}\right.$ po.d. $)$, the scar width was slightly decreased with all techniques but glue. Statistically significant differences were observed between glue compared to intradermal B and intradermal C. Judging from the width of the scar, the intradermal technique with burying of the knots and the use of $4 / 0$ poliglecaprone 25 , and the intradermal technique without burying of the knots and with the use of $4 / 0$ polypropylene were the best during the entire study period due to the narrower scar formed. The staples application had moderate width scar formation from the $24^{\text {th }}$ until the $365^{\text {th }}$ po.d., while the application of n-butyl tissue adhesive had the worse outcome because it created a wide scar from the $9^{\text {th }}$ until the $365^{\text {th }}$ po.d. So, if the formation of a narrow scar is desirable, the intradermal suture pattern with burying of the knots seems the best solution, whilst staples combine speed and a quite narrow scar.

Similar findings were observed by Vipond \& Higgins (1991), who examined the skin healing after application of intradermal techniques with polypropylene without burying of the knots and with polydioxanone with burying of the knots. They found no differences in the scar width between the techniques three months after suturing.

Gouletsou (2014) compared the scar width between the intradermal techniques with burying of the knots (without subcutaneous suturing) by using absorbable monofilament suture materials of different diameters (4/0 poliglecaprone $25,3 / 0$ poliglecaprone 25 and 4/0 polyglitone 6211 sutures) and the simple interrupted pattern (without subcutaneous suturing) by using 3/0 poliglecaprone 25 in $12 \mathrm{~cm}$ surgical skin closed incisions in dogs. The narrowest scar was observed with the intradermal techniques using 4/0 suture materials (poliglecaprone 25 and polyglitone 6211). Gouletsou (2014) also observed that during the first 40 po.days the scar width increased with all the intradermal techniques. In the present study, the scars remained thin with the intradermal techniques until the $21^{\text {st }}$ po.d, while from the $24^{\text {th }}$ until the $63^{\text {rd }}$ po.d., the scar width slightly decreased with the intradermal technique with burying of the knots and slightly increased with the intradermal technique without burying of the knots. Probably, the 2-layer closure of the incisions, with subcutaneous and intradermal suturing, improved the scars' width during this period. 


\section{Abscessation or inflammation and exudate}

In the present study, few cases of mild inflammation were observed with all techniques, whilst wound dehiscence or exudate discharge was not observed in any of them. Most of the cases occurred with intradermal B, whilst only one case was observed with intradermal C. Inflammation was usually observed the first postoperative days and lasted 1-4 days, except for one wound sutured with intradermal B, where the mild signs of inflammation lasted two months.

Gatt et al (1982) compared wound infection after suturing skin incisions with staples, or with $2 / 0$ polypropylene interrupted vertical mattress sutures, or with $3 / 0$ coated polyglactin interrupted vertical mattress sutures, whilst administering antibiotics preoperatively, and six and twelve hours postoperatively, and observed that wound infection was more intense in the staple and polypropylene groups. Johnson et al. (1997) evaluated 242 patients with sternal and saphenous vein harvest wounds, which were closed half way with staples and half with intradermal sutures without burying the knot (polypropylene monofilament suture), and observed that infection was lower with the intradermal suture technique in both locations however the difference was not statistically significant. Furthermore, no statistically significant differences in infection were observed by Nagpal et al (2004) on intradermal B and $7^{\text {th }}$ po.d., when comparing cyanoacrylate (n-butyl-) with suture material (3-0 silk percutaneous interrupted sutures) used in the closure of surgical skin wounds. Khan et al (2006) compared skin closure using staples, subcuticular 3.0 absorbable poliglecaprone suture and 2-octyl tissue adhesives in patients undergoing total hip and total knee replacement and observed no statistically significant differences in infection and abscessation (early and late wound complications). According to Iavazzo et al (2011), the use of staples for surgical wound closure was associated with significantly fewer wound infections, compared with the use of sutures, in patients undergoing obstetrics or gynecological, general, head/neck, and vascular operations, as well as emergency surgical procedures. No statistically significant differences in infection were observed by Eggers et al (2011), who compared four wound closure techniques (staples, 2-octyl- and n-butyl- tissue adhesives and 4/0 poliglecaprone 25) following total knee arthroplasty. Finally, Gouletsou (2014) observed more severe cases of inflammation and microabscess formation in intradermal suture patterns until the $35^{\text {th }}$ po.d, which, in 4 cases, were obvious- for 3-5 days, whilst in 3 cases for 15-30 days. This can probably be explained by the fact that no antibacterial drugs were administered postoperatively to these animals. On the contrary, the antibacterial drugs that were given to the animals of the present study, during the first 10 postoperative days, might have contributed to the low incidence of inflammation. It might be concluded that, even in noncontaminated clear surgical wounds, postoperative administration of antibacterial agents may reduce wound inflammation and prevent microabscess formation, that otherwise might occur.

\section{Comedones}

In the present study, comedones were observed on only three occasions in two animals. The first one was observed 77 days postoperatively in an incision closed with staples, 
whilst the other two were observed 35 and 42 days postoperatively in the same incision closed by intradermal with burying of the knot. Comedones have been connected with post suturing scars in only two references, i.e. Webster et al (1985), who reported comedones after suturing eyelid skin with catgut, andGouletsou (2014), who, 20 to 90 days postoperatively, observed comedones in almost all wounds sutured with intradermal patterns. Smeak (1992) suggested that epithelial cysts may form after traumatizing the stratum basale of epidermis or hair follicles during the passage of needle and suture, and Gouletsou (2014) assumed that this could be an explanation for the presence of comedones at the scars observed in her study. However, since in the present study only minimal comedones were observed, it should be suggested that probably antibiotics' administration and elimination of inflammation might also play a role in their absence.

\section{Scar hyperpigmentation}

The alteration of scar color was another characteristic of skin healing in the present study. In the majority of animals with all techniques, from the $35^{\text {th }}$ until the $180^{\text {th }}$ po.d., and in some animals until the $365^{\text {th }}$ po.d., the scar acquired a darker color than that of the adjacent skin. Afterwards, the scar's color became lighter and didn't differ from the color of the adjacent normal skin. From the $35^{\text {th }}$ until the $180^{\text {th }}$ po.d., the scar hyperpigmentation was more intense with staples and glue than with intradermal $B$ and intradermal C, the difference beeing statistically significant. Then, until the $365^{\text {th }}$ po.d., the color of the scar was darker with glue in comparison to staples, while it was very mild with intradermal $\mathrm{C}$ and no color difference was observed with intradermal B. During this period, a statistical difference was revealed between glue in comparison to intradermal B and intradermal C.

According to Swaim and Henderson (1997) and Hosgood (2006), the first signs of pigment deposition in the skin become visible 1-2 weeks postoperatively, although the maximum concentration of melanocytes is observed several months later. The melanogenesis depends on both exogenous and endogenous agents, such as alphamelanochrostikotropine (a-MSH) and adrenocorticotrophic (ACTH) hormone, vitamin D3, interleukins or other cytokines, which influence the process of melanin production from melanocytes (Miller et al 2013a, b). The change of scar's color was observed approximately on the $4^{\text {th }}$ postoperative week, probably due to the action of produced cytokines, which stimulated the production of melanin (Rudolph et al 1973, Matsumoto et al 1996). After this period the color of most scars became normal, except a few in which it became darker. In this study, both the intensity and the duration of hyperpigmentation were significantly different between the techniques. The pigmentation intensity of the scars was higher with both staples and glue, while the duration of hyperpigmentation was longer with glue. On the contrary, with intradermal suture patterns, pigmentation was milder and lasted less time. Gouletsou (2014) also reported hyperpigmentation at the scars after skin suturing. She observed that the scars were getting darker than the adjacent normal skin from the $28^{\text {th }}$ po.d. until the $180^{\text {th }}$ po.d, an in some cases until the $365^{\text {th }}$ po.d., in most of the wounds sutured with intradermal suture pattern or simple interrupted pattern; however, no 
statistically significant differences were observed between techniques. In wound sutured with simple interrupted pattern, hyperpigmentation was also found at the cross scaring formations (Gouletsou 2014), as was the case in the present study with staples application.

\section{Wound dehiscence}

Only one case of wound dehiscence was observed in an incision closed with staples two days after staple removal and it was 0.8 centimeters long. Perhaps staples should have been removed some days later so as the tensile strength of the healing wound would have been better. However, no dehiscence occurred to incisions closed with tissue glue. The use of subcutaneous suture in the present study might have contributed to this result because some studies report an increased incidence of wound dehiscence when tissue glue alone is used for skin closure (van den Ende 2004, Chow et al 2010). In a population control programme for cats, Faria et al (2005), when comparing two closure techniques (3-0 nylon simple interrupted sutures and n-butylcyanoacrylate tissue adhesive) for ovariohysterectomy, observed no statistically significant difference in wound dehiscence in cats held in semi-free environment as compared to the confined animals. Obermair et al (2007), when comparing the staples application and the intradermal techniques with burying of the knots, by using poliglecaprone 25 and poliglecaprone 6211 suture materials in gynaecological abdominal surgery, observed wound dehiscence in six (6/90) patients, two assigned to staples, three assigned to poliglecaprone 6211 and one assigned to poliglecaprone 25. Eggers et al (2011), when comparing four wound closure techniques (staples, 2-octyland n-butyl- tissue adhesives and 4/0 poliglecaprone 25) during total knee arthroplasty, observed dehiscence with slight wound separation (3 $\mathrm{mm}$ or less), that was approximately $6 \%$ for the adhesive and suture cohorts and $11 \%$ for the staples, but without any statistical differences.

\section{Cross scaring formation}

As expected, cross scaring formation was observed only when staples were used. The cross scaring formations were a consistent finding in all animals and were observed immediately after staples removal. The cross scaring formations were intense until the $21^{\text {st }}$ po.d., less pronounced from the $24^{\text {th }}$ until the $63^{\text {rd }}$ day and absent from the $70^{\text {th }}$ until the $365^{\text {th }}$ day. Similar were the findings of Gouletsou (2014), who observed cross scaring formation at the wound area after using simple interrupted pattern. In her study, cross scaring formations were present from the $20^{\text {th }}$ to the $180^{\text {th }}$ po.d.; however in some wounds, it persisted for three years after suturing. Crickeler (1958) reported that cross scars are probably the result of stricture of skin inside the stitches' loop, which induces ischemia and scar formation. Making the stitches loose helps to eliminate the problem; however subcutaneous suture pattern should be applied first in order to bring skin edges in apposition (Adams et al 2003). In the present study subcutaneous suture was performed first in order to minimize tension at the wound 
margins and special care was given to place staples correctly. However, tissue strangulation and cross scar formation were not prevented.

Cross scar formation was the main disadvantage of staples; otherwise, their application was very quick, not technical demanding, and followed by a good cosmetic outcome. Furthermore, the fact that the scar marks fade with time and become less visible 70 days postoperatively makes cross-scaring of minor importance. Anate (1991), Orozco-Covarrubias and Ruiz-Maldonado (1999) and Parell and Becker (2003) also observed cross-scar formation after simple interrupted suture pattern, that faded after the $90^{\text {th }}$ po.d.

\section{Total clinical evaluation}

It is obvious that each technique influences wound healing in a different way. Some parameters of clinical evaluation are favorable for one technique while others are favorable for another. In the present study, in order to have an overall view of the total clinical evaluation of each technique, some of the scores of clinical parameters were summed up and a total clinical score for each one of the five periods was calculated.

As was expected, total clinical scores improved over time with all techniques. In period A the improvement was larger with the intradermal suture patterns. On the contrary, with staples and glue, the clinical evaluation was improved after the period C.

In period A $\left(1^{\text {st }}-8^{\text {th }}\right.$ po.d.), the total clinical scores of intradermal B and intradermal $\mathrm{C}$ were better than those of staples and glue. However, the largest, but not statistically significant, difference was observed between intradermal B and staples. The continuous intradermal suture patterns (intradermal B and intradermal C) had better scores due to the milder skin thickening, the thinner scar and the smaller erythema at the wound area compared to the staples and glue.

In_period $\mathrm{B}\left(9^{\text {th }}-21^{\text {st }}\right.$ po.d. $)$, the total clinical scores of intradermal $\mathrm{B}$ and intradermal $\mathrm{C}$ continued to be better than those of glue and staples due to the narrow scar width and the mild skin thickening. Furthermore, during period B, the score of staples was statistically different from that of the other techniques, due to the cross scaring formation and the intense skin thickening at the wound area.

In period $C\left(22^{\text {th }}-63^{\text {rd }}\right.$ po.d.), the intradermal suture patterns had better total clinical scores compared to the scores of glue and staples. Moreover, staples had a statistically significant worst score compared to the scores of intradermal B and intradermal C, as cross scaring formations persisted.

In period $\mathrm{D}\left(64^{\text {th }}-180^{\text {th }}\right.$ po.d.), the intradermal suture patterns had better total clinical scores compared to glue and staples. The score of staples was still statistically worse compared to those of intradermal B and intradermal C. Although the cross scaring marks were absent, the larger scar width and the more pronounced scar pigmentation adversely affected the score of staples in comparison to intradermal B and intradermal $\mathrm{C}$, in which the scars were thinner with lower or absent pigmentation. It should also be mentioned that the more pronounced pigmentation of the scars with staples adversely affected the total clinical score in comparison to intradermal B and intradermal C. 
Finally, in period E (181 $11^{\text {st }} 365^{\text {th }}$ po.d.), the total clinical scores of intradermal B and intradermal $\mathrm{C}$ continued to be better in comparison to those of staples and glue. A significant difference was observed between intradermal B and intradermal C compared to glue. This change observed during the mature phase between staples and glue was due to the improvement of both width and pigmentation of the scar with staples in comparison to glue, with which the scar width continued to be large and with pigmentation. Between the intradermal suture patterns, the total clinical score of the pattern with burying the knots was better in relation to the pattern without burying the knots.

Gouletsou (2014) also compared the total clinical evaluation between the intradermal suture technique pattern with burying the knots using absorbable monofilament sutures of different diameters and materials (4-0 poliglecaprone 25 , 3-0 poliglecaprone 25 and 4-0 polyglitone 6211) and the simple interrupted suture pattern using 3-0 poliglecaprone 25, all performed in $12 \mathrm{~cm}$ long surgical skin incisions in dogs. The intradermal techniques performed with 4-0 suture materials (poliglecaprone 25 and polyglitone 6211) were evaluated clinically as the best from the $3^{\text {rd }}$ po. month until the $1095^{\text {th }}$ po.d.

\section{Ultrasonographic evaluation}

In order to evaluate ultrasonographically the skin healing process over time, B mode ultrasonography was used and the wound area was estimated. The surgical closure of the skin edges created a compartment that was filled with a serohemorrhagic exudate derived from blood plasma, as a product of the acute inflammation caused by surgery that was $\mathrm{u} / \mathrm{s}$ recorded as a hypoechoic to anechoic small region. On the first postoperative days, the lower limits of the wound area were difficult to be identified due to the similar echogenicity of the subcutaneous tissue to the oedematous wound area. As wound healing progressed, the wound area size and the tissue oedema diminished, and the progressive collagen deposition altered echo intensity, making wound boundaries more complex but not indistinguishable.

In period A $\left(1^{\text {st }}-8^{\text {th }}\right.$ po.d. $)$, no statistically significant difference was revealed between techniques; however, wound area (in $\mathrm{mm}^{2}$ ) was $\mathrm{u} / \mathrm{s}$ estimated to be smaller with intradermal B compared to the other techniques. Staples and glue had the largest values of wound area, something that was in accordance with the larger skin thickening observed clinically. Obviously, during this period, the dominant characteristic of the wound was oedema, the intensity of which depended on the technique used.

In period B $\left(9^{\text {th }}-21^{\text {st }}\right.$ po.d.), although the wound area was substantially decreased with all techniques, it was $\mathrm{u} / \mathrm{s}$ estimated to be smaller with staples in comparison to intradermal C, glue and intradermal B, while with the latter two techniques it was the largest. Statistically significant difference was revealed only between intradermal B and staples. During that time period, staples had been removed, reducing wound oedema, whilst with intradermal B the implanted suture material continued to produce tissue irritation. From the middle of this period, the wound area with all techniques was reduced almost by half in relation to the initial measurements. 
In period C $\left(22^{\text {th }}-63^{\text {rd }}\right.$ po.d.), wound size was even more decreased with all techniques and was u/s estimated to be smaller with staples and intradermal $\mathrm{C}$ and larger with glue and intradermal B. Statistically significant difference was revealed only between staples and intradermal $\mathrm{B}$. The $\mathrm{u} / \mathrm{s}$ measured wound area was larger with intradermal B compared to the intradermal C, due to the continuous tissue irritation from the implanted suture material.

In period D $\left(64^{\text {th }}-180^{\text {th }}\right.$ po.d.), the wound size was estimated to be smaller with intradermal C compared to the staples, intradermal B, and glue. Statistically significant difference between the techniques was not revealed. During this period, and specifically up to the $120^{\text {th }}$ po.d., although the $\mathrm{u} / \mathrm{s}$ wound area was small with intradermal B and glue, it was still distinctly imaged, due to the hypoechoic appearance of the newly formed dermis. On the contrary, it was imaged with difficulty with intradermal $\mathrm{C}$ and staples, because the echogenicity of the wound area was similar to the adjacent normal skin's. After the $150^{\text {th }}$ po.d., in almost all incisions with all techniques, the wound area was imaged as a small and of low-sound intensity area inside the normal echogenic dermis and below the more echogenic epidermis.

In period $\mathrm{E}\left(181^{\mathrm{st}}-365^{\text {th }}\right.$ po.d.), although the $\mathrm{u} / \mathrm{s}$ measured wound area was minimal with all techniques, it was estimated as smaller with staples in comparison to intradermal C, intradermal B and glue; however, a statistically significant difference between the techniques was not revealed, while some difference was observed between staples and glue $(\mathrm{p}=0.03)$.

Generally, from the $9^{\text {th }}$ until the $365^{\text {th }}$ po.d., the smaller wound area was constantly recorded with the staples and with the intradermal $\mathrm{C}$, probably because there was no suture material inside the wound to induce tissue reaction. On the contrary, with the intradermal B, the concurrent tissue reaction to suture material present in the wound area, up to the $119^{\text {th }}$ po.d., contributed to the larger wound area. With the tissue glue, the loose apposition of the wound edges might have contributed to the larger wound area, which was constantly larger in relation to that observed with the other techniques. It should also be mentioned that on the $365^{\text {th }}$ po.d., the wound area could not be detected in a few segments of some wounds with all techniques, suggesting complete skin repair.

As a conclusion, as far as $\mathrm{u} / \mathrm{s}$ estimated surgical wound size is concerned, the technique that had the smaller wound area over all time periods was intradermal B. Staples, that showed the largest wound area during the first week, afterwards had the smallest until the end of the experiment.

\section{Histological evaluation}

Histological examination gives detailed information on the healing process of the traumatized skin that may not be revealed by any other method. It shows the presence of necrosis, the causative agent of skin thickening, the presence of inflammation and the types of cells involved in it, the presence of suture material and the tissue reaction to it, the epithelial gap and thickness at the wound area, the scar width, and the way 
tissues heal by collagen production, presence of fibroblasts and angiogenesis. It is also important that all the above parameters are countable, and, as a result, statistical significance may be found. However, in clinical settings that animals or humans are used, performing multiple skin biopsies during healing is very difficult or even unfeasible. This is the reason why only a few studies that provide histological information on skin healing can be found, and even less have been performed on the canine skin. The most detailed study on canine skin healing was contacted by Gouletsou (2014), who investigated healing of skin by clinical examination and histopathology, after suturing it with intradermal suture pattern or simple interrupted pattern and using sutures of different diameter and material.

\section{Necrosis}

Necrosis was not observed in any tissue sample with any of the techniques, confirming the clinical evaluation. The absence of necrosis was expected because the incisions were performed under aseptic conditions, skin perfusion was not affected since no large vessels were sectioned, the skin was sutured without any tension, and antimicrobial drug therapy was given post-operatively. Furthermore, no necrosis was observed in the area where occlusions of the glue substance inside the dermis were noticed. Neither Gouletsou (2014) observed any necrosis in her study.

\section{Oedema}

Oedema was a constant finding on the $7^{\text {th }}$ po.d. When oedema was observed on the $14^{\text {th }}$ or $28^{\text {th }}$ po.d., it was accompanied by inflammation at the wound area. During period A, no significant difference was observed between techniques. Moderate oedema was present at only one sample with glue and intradermal $\mathrm{B}$, while mild oedema was present at four samples with staples and at five samples with glue, intradermal B and intradermal C. During period B, mild oedema was present at a single sample closed with staples. It seems though, that oedema is an expected finding during the first week of healing and is not affected by the technique of skin closure. However, it is connected with the presence of inflammation at the wound area, and in that case, may last even one-month post surgery. Gouletsou (2014) also found oedema in almost all wounds examined on the $7^{\text {th }}$ po.d. with all the techniques, in $17 / 40$ samples on the $14^{\text {th }}$ po.d. and in $5 / 40$ samples on the $28^{\text {th }}$ po.d. In her study, all the cases of oedema after the second postoperative week were found in samples that inflammation was present (Gouletsou 2014). Since she gave no antimicrobial drugs, inflammation and oedema were more common and lasted a longer period of time (Gouletsou 2014). Papazoglou et al (2010), evaluated oedema in six cats subjected to skin suturing with intradermal suture pattern with either the copolymer of glycolide, caprolactone, and trimethylenecarbonate, or with polypropylene suture with clips. In their study oedema was present in all the incisions sutured with the first suture on the $2^{\text {nd }}$ po.d., but subsided on the $7^{\text {th }}$ po.d. In contrast, swelling of incisions was present in 3 cats sutured with polypropylene on the $2^{\text {nd }}$ po.d. but subsided in all cats on the $2^{\text {nd }}$ po.d. 


\section{Inflammation}

With all techniques, at the $7^{\text {th }}$ po.d., the main histopathological finding in the wound area was leukocyte-infiltration. In most cases, macrophages predominated, whilst lymphocytes were observed less frequently. Neutrophils were observed either around the suture material or as a cluster in the dermis or subcutaneous tissue. The presence of macrophages and lymphocytes was compatible with the first stage of skin healing, although one week after surgery the inflammation normally should begin to subside as the skin repair stage starts. The prolonged presence of neutrophils into aseptic wounds after the $1^{\text {st }}$ po.d. indicates either the presence of foreign body, such as suture material, or infection. Neutrophils can adversely affect the healing process or cause excessive fibrosis (Satish and Kathju 2010). In the present study, no significant difference of infiltration with leukocytes was observed between the techniques on the $7^{\text {th }}$ po.d., indicating that the initial stage of skin healing was affected neither by the presence of suture material nor by the technique and that the presence of leukocytes was probably a consequent of the normal healing process.

On the $14^{\text {th }}$ po.d., inflammation persisted as before, whilst on the $28^{\text {th }}$ po.d., it subsided with all techniques. Between the thinner than normal collagen bundles, limited numbers of macrophages, fibroblasts, lymphocytes, and mast cells were observed. The intensity of inflammation did not differ between techniques. Traumatic furunculosis foci were present. Fragments of hair penetrated the wound area either during skin cutting and handling or were transported out of hair follicles during the passage of the needle through the dermis. Careful removal of hair fragments with a vacuum cleaner after hair trimming could reduce the risk of traumatic furunculosis.

On the $180^{\text {th }}$ and $365^{\text {th }}$ po.d., inflammation subsided almost completely in the wound area, while a scar without glands or hair follicles was present.

No statistically significant difference in inflammation score was observed between the techniques. However, more cases of mild or severe inflammation were observed with intradermal suturing with burying of the knots (21) and with use of glue (20) than with the other two techniques (18 each).

Skin stapling caused minimal or mild inflammation at the incision area. On the $7^{\text {th }}$ and $14^{\text {th }}$ po.d. infiltration by a small number of neutrophils, macrophages, and fibroblasts was observed, which is consistent with the healing process of the wound. Nodular accumulation of macrophages and lymphocytes at the subcutaneous tissue in a few samples was probably caused by the presence of suture material nearby or by traumatic furunculosis. From the $28^{\text {th }}$ po.d. onwards, no inflammation was noticed. It seems that the use or inert metal staples that are removed 10 days po.d. minimizes foreign body inflammatory reaction in the wound area.

Skin glue application caused mild to medium inflammatory reaction to the wound area. From the $7^{\text {th }}$ to the $28^{\text {th }}$ po.d. the main finding was infiltration by neutrophils, macrophages, lymphocytes, and fibroblasts. Some foci with traumatic furunculosis and some comedones were also observed. Afterwards, no inflammation was noticed. The most important finding was that in spite of care during application, small quantities of glue were deposited below the epidermis, initiating inflammation in the dermis, a finding that persisted until the $365^{\text {th }}$ po.d. According to Mobley et al (2002), 
glue implanted subcutaneously may induce chronic inflammation including oedema, erythema, pain, or purulent drainage. Toriumi et al (1991) demonstrated significant inflammation and even tissue necrosis attributed to exposure of butyl-2-cyanoacrylate to well-vascularized subcutaneous tissues. In contrast, subcutaneous placement of the adhesive within the confines of two cartilage surfaces without exposure to the surrounding vascular tissue failed to elicit a significant inflammatory response. Toriumi et al (1990) also showed that inadvertent use of this agent below the skin and in exposed subcutaneous tissue can result in moderate tissue damage and even necrosis

According to the above mentioned, insertion of the glue into the subcutaneous tissue may potentially increase the chance of delayed wound healing or inflammation and necrosis. However, as in the present study, the inflammatory reaction around glue inclusions presented only 3-6 layers of cells, it seems that the inclusions' small dimensions could not substantially affect wound healing. Furthermore, no necrosis, intense inflammation or purulent drainage was observed.

With the intradermal $\mathrm{B}$, on the $7^{\text {th }}$ and the $14^{\text {th }}$ po.d., the main finding was infiltration by neutrophils, macrophages, and fibroblasts at the site of the incision. Minimal to mild tissue reaction, consisting of macrophages and fibroblasts, was usually observed around the suture material. The upper dermis next to the wound area was infiltrated by small numbers of macrophages and few mast cells. From the $28^{\text {th }}$ po.d. onwards, no inflammation was noticed. It seems that the presence of suture material for a long time postoperatively enhances the presence of leucocytes at wound area, as it promotes a foreign body reaction. However, no statistically significant difference was observed between this technique and the others that no material was implanted. Gouletsou (2014) also observed that medium to large numbers of neutrophils and macrophages infiltrated the wound area during the first 14 days postoperatively when the wound was sutured by intradermal suture pattern with burying of the knot. However, in her study, more cases of severe inflammation (perivascular, nodular or diffuse) were detected, probably because no antibiotics were administered postoperatively.

With the intradermal $\mathrm{C}$, on the $7^{\text {th }}$ and $14^{\mathrm{t}} \mathrm{h}$ po.d. , the main finding was infiltration by small numbers of neutrophils, macrophages, and fibroblasts. Mild tissue reaction, consisting of macrophages and fibroblasts, was usually observed around the suture material. This was rather expected, as polypropylene suture material produces a small cellular reaction (Boothe 2003). The upper dermis next to the wound area was infiltrated by small numbers of macrophages and few mast cells. From the 28th po.d. onwards no inflammation was detected. It seems that the intradermal suture pattern with clips promotes epithelial bridging that protects the wound from bacterial contamination, thus limiting inflammation. Furthermore, as the suture material is removed on the $10^{\text {th }}$ po.d., no mechanical irritation is present to promote inflammation during the later stage of wound healing, as may be the case with intradermal B.

Papazoglou et al (2010) evaluated inflammation in six cats following suturing of the skin with intradermal suture pattern either with a copolymer of glycolide, caprolactone, and trimethylene-carbonate sutures, or with polypropylene suture with 
clips, and noticed that cellular reaction was moderate to severe in most incisions, regardless of the closure technique. This might be due to the trauma produced by placement of the needle, and due to the reaction to the suture material.

\section{Epidermal thickness at the wound area}

In the present study, in general, during the first po. days, in all techniques, the epidermal thickness at the area of wound healing was increased in relation to the adjacent healthy skin. Increased epidermal thickness was not restricted to the newly formed epithelium but was also extended to the intact wound edges. The intense proliferation of cells at wound borders intended to replace losses due to continued movement of epidermic cells to the epidermal deficit (Martin 1997).

In period A, median thickness of epidermis at the area of wound healing was approximately 2.5 times that of the adjacent healthy epidermis with staples, intradermal $\mathrm{B}$, and intradermal $\mathrm{C}$, whilst it was 3.5 with glue, with the last being significantly different from intradermal $C$. The same, but to a lesser extent, was observed in period $\mathrm{B}$, during which median thickness of epidermis at the area of wound healing was 2 times that of the normal epidermis with staples, intradermal B, and intradermal $\mathrm{C}$, whilst it was 3 times that of the normal with glue. This difference persisted in period $\mathrm{C}$, with the median thickness of epidermis at wound area being significantly larger with glue compared to staples and intradermal C. No difference was observed from period D onwards, as thickness of the epidermis at the wound area was almost similar to that of the normal adjacent skin. It seems that there are no differences between staples, intradermal $\mathrm{B}$ and intradermal $\mathrm{C}$ on the epithelial thickness and that the initially increased epithelial thickness subsides one month postoperatively. On the contrary, with glue, the increase in epithelial thickness is significantly higher than that of the other techniques and lasts for a longer period of time. It is possible that presence of glue substance at the wound area provoked the increased proliferation of the epidermis at wound borders, in an effort of the epithelium for optimal epithelialization. It is also possible that chemical or thermal irritation of the epidermis due to polymerization of the glue resulted to an intense increase of epithelial thickness at wound area so that a longer time period was needed in order to restore to normal.

Papazoglou et al (2010), observed that on the $9^{\text {th }}$ po.d thickness was larger in 4 out of 6 sections which included glycolide, caprolactone, and trimethylene-carbonate, whilst was normal in all sections which included polypropylene. In the present study, the epithelial thickness in incisions sutured with polypropylene returned to normal one month postoperatively, however, the method of measuring the epithelial thickness differs between this study and that of Papazoglou et al (2010). Furthermore, species differences may contribute to this discrepancy.

Gouletsou (2014) also observed two- to three-fold increases in epithelial thickness on the $7^{\text {th }}$ po.d with all techniques used, and in some occasions reached a five-fold increase. The increase in her study subsided on the $28^{\text {th }}$ po.d and was smaller in incisions closed by intradermal technique with burying of the knot. 


\section{Epithelial gap}

Epithelial gap was observed at only three samples with glue on the $7^{\text {th }}$ po.d., while epithelial bridging had been completed on the $14^{\text {th }}$ po.d. in all samples. It seems that the use of glue may delay epithelial bridging, probably due to the deposit of small quantities of glue below the epidermis, especially when the edges of the wound are not in close contact during glue application. This may initiate inflammation of the dermis or delay the migration of the cells of the epidermis. Gouletsou (2014) observed that epithelial bridging delayed more and that 25 out of 40 samples from all the techniques she used presented epithelial gap on the $7^{\text {th }}$ po.d. Perhaps in the present study, the use of antibiotics postoperatively contributed to the faster epithelialization observed in all techniques. Pope (1993) also found that the epithelialization was completed at the first postoperative days after surgical skin closure. Kirpensteijn et al (1997), who checked the epithelial bridging after suturing skin incisions with either intradermal suture with poliglecaprone 25 or polyglactin 910, also observed that all the wounds had been epithelialized on the $7^{\text {th }}$ po.d.; however they give no information about any antibiotic administration.

Papazoglou et al (2010) noticed that epithelial bridging was completed at the $9^{\text {th }}$ po.d. in all incisions closed by polypropylene but in only 1 out of 6 incisions closed by the other technique.

\section{Histological scar width}

In all time periods, no significant differences were revealed between the techniques in the histologically evaluated scar width. In period A, scar width was much larger with glue compared to the other techniques, with staples and intradermal B presenting the narrowest scars. In period B, scar widths with staples and glue were equal, whilst intradermal B presented the narrowest scar. In period $\mathrm{C}$ median values of scar width were almost equal between techniques, with staples, glue and intradermal $\mathrm{C}$ having higher $75 \%$ interquartile values compared to intradermal B. In period D, scar width decreased in all techniques with the exception of glue. In period E scar width remained the same as in period $\mathrm{D}$ in all techniques with the exception of staples, in which it was further decreased.

It seems that scar width gets narrower over time with all techniques; however, the improvement was poorer with glue than with the other techniques. Furthermore, the scar width with glue was larger than with the other techniques throughout the whole experimental time, indicating that glue should not be used when a narrow scar is mandatory.

The only other study of histologically evaluated scar width is that of Gouletsou (2014), who observed that scar width was larger in incisions sutured with simple interrupted stitches in comparison to those sutured with intradermal suture patterns. Her findings were constant the whole study period, which was three years postoperatively. In that study, intradermal suture pattern with burying of the knots produced a scar of approximately $0.7 \mathrm{~mm}$ in width, which is slightly larger than the values found in the present study, i.e. $0.45-0.56 \mathrm{~mm}$. This difference may be partly the 
result of antibiotics administration and of the less intense inflammation observed in the present study.

\section{Suture material presence and tissue reaction around the suture material}

The presence of suture material or its initial position was identified at 30 samples in intradermal B and intradermal C and of these, poliglecaprone 25 suture material was found in 24 samples, whilst polypropylene suture material was found in 6 samples. Finding the suture material was not always possible, as in some cases it had been removed from the tissue samples during cutting and staining procedures. In two cases the place of the initial implantation position was found whilst the suture material had been moved to a nearby position. Generally, tissue reaction around polypropylene was minimal and composed of a few cell layers containing macrophages and fibroblasts. With intradermal $\mathrm{B}$, on the $7^{\text {th }}, 14^{\text {th }}$ and $28^{\text {th }}$ po.d., tissue reaction was evaluated as minimal to mild, containing macrophages and fibroblasts. Finally, in one case, around poliglecaprone 25 suture, there was a strong reaction with an accumulation of large numbers of inflammatory cells such as neutrophils, macrophages, and lymphocytes; multinuclear giant cells were observed more rarely. It was characteristic that in a sample in which the suture material was located inside the lumen of a hair follicle, infiltration by macrophages, leukocytes, and fibroblasts was observed around it.

From the $180^{\text {th }}$ po.d. onwards, the area where the suture material had been implanted, was impossible to be detected at any tissue sample. It has been reported that after full absorption of the suture material, the area where it was located is characterized by the presence of macrophages surrounded by fibroblasts (Postlethwait et al 1975, Salthouse 1983, Bezwada et al 1995). However, in the present study, accumulations of macrophages were only observed around hair and debris, probably as a result of traumatic furunculosis.

According to Bezwada et al (1995), implantation of poliglecaprone 25 into the muscular tissue of rats provoked slight or minimal inflammatory reaction, characterized by the presence of macrophages and fibroblasts, fewer lymphocytes and plasma cells, small numbers of polymorphonuclear leucocytes, and occasional giant cells. These cells gradually diminished in concentration during a 119-day study period, with the suture being absorbed between 91 and 119 days post implantation. Perhaps implantation of the suture to the muscular tissue resulted in a less severe inflammatory reaction in comparison to the present study.

Van Heerden (2005) compared histologically the inflammatory response to polyglitone 6211 and poliglecaprone 25 in intracuticular skin closure in a rat model at the $2^{\text {nd }}$ and $10^{\text {th }}$ po.d. and observed no difference between the two sutures. In that study, neutrophils predominated at the $2^{\text {nd }}$ day and macrophages at the $10^{\text {th }}$ po.d. In half of the samples, inflammatory reaction was slight and in the rest mild or severe, without any difference between the techniques.

Ribeiro et al (2005) evaluated, through a clinical and histopathological analysis, tissue reactivity caused by two monofilament sutures, i.e. nylon and poliglecaprone 25, used for internal external sutures in rats. They observed mild or intense inflammatory reaction in all animals at the $7^{\text {th }}$ and $14^{\text {th }}$ po.d., that subsided afterwards. 
They also observed fibrosis around the suture material in $70 \%$ of the samples at the $7^{\text {th }}$ po.d. and in $40 \%$ of them at the $21^{\text {st }}$ po.d. A small number of giant cells were observed at the $14^{\text {th }}$ and the $21^{\text {st }}$ po.d. The external sutures (poliglecaprone 25) showed a greater tissue reaction that decreased while the thread was being absorbed.

Molea et al (2000) conducted an investigation on 72 rats to compare three types of monofilament absorbable suture material i.e. polydioxanone, poliglecaprone 25 , and glycomer 631, with respect to their clinical characteristics, tissue inflammatory reaction and suture absorption times. They evaluated the animals 15 and 30 days and 3 and 6 months after suturing the skin with simple vertical intradermal stitches. Their results indicated that poliglecaprone 25 and glycomer 631 sutures are less reactive than polydioxanone. Tissue reaction around the suture material was mild at the $15^{\text {th }}$ and $30^{\text {th }}$ po.d. and had completely subsided at the $3^{\text {rd }}$ and $6^{\text {th }}$ po. month.

Andrande et al (2006) studied the subcutaneous tissue reaction to polyglactin 910, polydioxanone, poliglecaprone 25 and chromic gut on the $1^{\text {st }}, 2^{\text {nd }}, 3^{\text {rd }}, 7^{\text {th }}$ and $14^{\text {th }}$ po.d. after suture implantation in 20 rats. As far as poliglecaprone is concerned, on the $2^{\text {nd }}$ po.d. they found diffuse infiltration of a few lymphocytes among the collagen fibers around suture material. At the $3^{\text {rd }}$ post-implantation day, mild granulation tissue was present, associated with a small population of inflammatory cells. The fibers began to organize themselves on the $7^{\text {th }}$ po.d., amid granulation tissue in continuous arrangement along the interface that contained macrophages, and scar tissue with elongated fibroblasts was formed on the $14^{\text {th }}$ day. In the present study, no fibrosis was observed around suture material on the $7^{\text {th }}$ po.d., even if fibroblasts were detected.

\section{Fibroblasts presence, collagen deposition, and angiogenesis}

In all periods presence of fibroblasts, collagen deposition and angiogenesis were not significantly different between the techniques.

On the $7^{\text {th }}$ po.d., the wound healing area was infiltrated by a large number of fibroblasts, while the number and the diameter of collagen fibers were small. Furthermore, neoangiogenesis, which is necessary for wound healing, was also observed and was reflected by the presence of erythema that was clinically observed. On the $14^{\text {th }}$ po.d. , the number of fibroblasts increased, while the collagen fibers were dense and the bundles slightly thicker. In the samples without inflammatory reaction present in the wound area, angiogenesis subsided by the $14^{\text {th }}$ po.d. On the $28^{\text {th }}$ po.d., the number of fibroblasts was high and the collagen fibers had further increased in number and size, while vasculature in the wound area was similar to that of the adjacent normal skin. On the $180^{\text {th }}$ po.d., fibroblasts decreased in numbers and the collagen fibers were significantly thicker. However, the thickness of collagen fibers in the healing area was clearly smaller than the thickness of those in the adjacent skin, and remained thinner, as far as the $365^{\text {th }}$ po.d. Gouletsou (2014) also observed that collagen fibers at the healing area were thinner than that of the adjacent skin even three years postoperatively.

Van Winkle et al (1975), who measured biochemically the collagen quantity in skin wounds closed with different suture materials, observed that collagen production 
was not affected by the type of suture material. Gouletsou (2014) also found no difference in the number of fibroblasts, collagen production and angiogenesis between the techniques used. Papazoglou et al (2010), when evaluated wound vascularity and collagen content in six cats after suturing their skin with intradermal suture pattern either with a copolymer of glycolide, caprolactone, and trimethylene-carbonate or with polypropylene suture with clips, observed no significant difference between the two techniques. However, it is possible that the scoring system of collagen production which was followed in the present study, after being established in many similar studies, would not be appropriate to estimate subtle differences in collagen production during wound healing by first intention.

\section{Total histological evaluation}

The total histological evaluation for each time period was recorded after summing up the individual scores of oedema, inflammation, tissue reaction around the suture material, thickness of the epidermis at the wound area and scar width.

In period A, a better total histological score was recorded with staples, intradermal $\mathrm{B}$ and intradermal $\mathrm{C}$ in comparison to glue. The later had the worse score due to larger scar width, larger epidermal thickness at wound area and presence of epithelial gap in a few cases. In period B, a better total histological score was recorded with intradermal B in comparison to staples, glue, and intradermal C, with significant difference between glue and intradermal B. In period C, a better total histological score was recorded with staples, intradermal B and intradermal C in comparison to glue and significant difference was revealed between glue and intradermal C. In period D, a better total histological score was recorded with staples, intradermal B and intradermal $\mathrm{C}$ in comparison to glue, in which the total histological score was slightly worse than before. In period $\mathrm{E}$, the total histological score was equal in all techniques.

Although no significant differences were observed in relation to collagen deposition, fibroblasts presence, angiogenesis and inflammation presence, between the techniques, the total histological score of the incisions closed with glue was worse until the $160^{\text {th }}$ day. Delayed epithelialization in a few cases and wider scar affected negatively the total histological score with glue in comparison to staples and intradermal suture patterns. Intradermal suture patterns were better due to optimal epithelialization and a smaller scar. However, on the $365^{\text {th }}$ po.d., when the only histological finding is scar width since all the rest subside, no histological differences can be observed between the techniques.

\section{Total evaluation of each technique}

After summing the scores of cosmetic, clinical, ultrasonographic and histological evaluation on the $7^{\text {th }}, 14^{\text {th }}, 28^{\text {th }}, 180^{\text {th }}$ and $365^{\text {th }}$ po. days, the total score of each technique was calculated, in an attempt to estimate the overall outcome of each technique over time. Therefore a total evaluation of each technique is necessary, even though the final conclusions should be managed with some concern.

Thus, on the $7^{\text {th }}$ po.d., intradermal B presented the best total score, followed by intradermal C, staples, and lastly, glue, which differed significantly from intradermal 
B. It seems that during the first postoperative week, the intradermal techniques accomplish better contact of skin edges and allow faster epithelialization, and therefore a better overall result is achieved.

On the $14^{\text {th }}$ po.d., intradermal $B$ had the best total score, followed by the intradermal $\mathrm{C}$, glue, and lastly, staples.

On the $28^{\text {th }}$ po.d., intradermal $C$ presented the best total score, followed by intradermal B, staples, and lastly, glue. One month postoperatively, the intradermal suture pattern with burying of the knot causes mechanical irritation of the healing area and foreign body inflammatory reaction, and this is probably the reason why the intradermal suture pattern with clips, in which the suture has been removed, shows a better overall score. Gouletsou (2014) also found that with intradermal suture patterns with burying of the knot, the good cosmetic outcome observed during the first postoperative period deteriorates during the $4^{\text {th }}-8^{\text {th }}$ postoperative week, and improves again afterwards, when the suture material is absorbed. However, the intradermal suture patterns, in general, have a better overall score than the other two techniques.

On the $180^{\text {th }}$ po.d., intradermal B presented the best total score, followed by the intradermal C, staples, and lastly, glue. Six months postoperatively, inflammation, being the result of either the healing process or foreign body reaction, had subsided, as the poliglecaprone suture material had been absorbed two months ago. The intradermal suture pattern with burying of the knots seems to have a better overall score than the other techniques, probably because it promotes better contact of skin edges and holds them together for a longer period of time, preventing expansion of the scar.

Finally, on the $365^{\text {th }}$ po.d., intradermal B still had the best total score, followed by the intradermal $\mathrm{C}$ and staples, and lastly, glue. However, no significant difference was revealed between any of them.

In the present study, the data revealed that the cosmetic, ultrasonographic, clinical and histological results were better with intradermal suture patterns. Furthermore, the results with tissue glue were worse than the other techniques. A possible explanation for this is that tissue glue sloughs off with the shedding of epidermal cells, and therefore cannot provide enough adhesion before adequate wound healing is achieved. Furthermore, when wound edges are not approximated well enough to prevent tissue glue from entering the wound, inflammation may be induced.

\section{Correlations}

Skin ultrasonography is a new technique used in studies performed in the canine skin and only a few studies have been published so far. The normal appearance of the skin of Beagles as seen with high-frequency ultrasound has been published by Mantis et al. (2014), where correlation with the histological appearance has been made. The present study is the first to use high-frequency ultrasonography in order to observe the way skin heals after the use of four skin closure techniques that aim in healing by first intention. Furthermore, in order to find out if high-frequency ultrasonography depicts 
the real ultrastructure of skin during first intention healing, a number of correlations have been made.

\section{Correlation between $\mathrm{u} / \mathrm{s}$ estimated wound area and histologically evaluated inflammation}

The correlation test between $\mathrm{u} / \mathrm{s}$ estimated wound area at the biopsy site and histologically estimated inflammatory reaction at the same area showed that there is statistically significant linear positive correlation between the two parameters. This means that the larger oedema and leucocytes infiltration due to inflammatory reaction at wound area, the larger the area with reduced echogenicity calculated ultrasonographically.

\section{Correlation between $\mathrm{u} / \mathrm{s}$ estimated wound area and histologically evaluated scar width}

The correlation test between $\mathrm{u} / \mathrm{s}$ estimated wound area at the biopsy site and histologically estimated scar width at the same area showed that there is statistically significant linear positive correlation between the two parameters. Therefore, the larger the $\mathrm{u} / \mathrm{s}$ estimated wound area, the larger the scar width calculated histologically. This correlation existed throughout the whole experimental period, which was much longer than the first postoperative days when inflammation is present. After inflammation subsided, ultrasonography detected the difference in collagen deposition, since collagen bundles were thinner than those of the nearby normal skin. However, on the $365^{\text {th }}$ po.d., the scar area had not been detected in all ultrasonography scans, probably because collagen density at wound area did not differ significantly from the nearby area.

\section{Correlation between $\mathbf{u} / \mathbf{s}$ estimated wound area and clinically evaluated skin thickening}

The correlation test between $\mathrm{u} / \mathrm{s}$ estimated wound area and clinically evaluated skin thickening showed that there is a statistically significant linear positive correlation between the two parameters, i.e. the larger the $\mathrm{u} / \mathrm{s}$ estimated wound area, the larger the skin thickening evaluated clinically by skin caliper. Based on this finding, it is feasible to calculate skin thickness at a surgically closed incision without the need to use skin caliper, which cannot be used at areas of small surface, at areas that the skin cannot be raised to create a fold, or when the procedure is painful.

\section{Correlation between $\mathrm{u} / \mathrm{s}$ wound area and clinically evaluated scar width}

The correlation test between $\mathrm{u} / \mathrm{s}$ estimated wound area and clinically evaluated scar width showed that there is a statistically significant linear positive correlation between the two parameters, i.e. the larger the $\mathrm{u} / \mathrm{s}$ estimated wound area, the larger the scar width measured by electronic caliper on the surface of the skin. However, one can say that ultrasonographic measurement of a scar area is favorable, as it evaluates the extent 
of the scar even when a scab covers the wound. Furthermore, ultrasonography depicts the extent of the scar under the surface of the epidermis, which is the only visible part during clinical examination. 
Institutional Repository - Library \& Information Centre - University of Thessaly 26/04/2023 13:56:30 EEST - 54.147.182.235 


\section{CONCLUSIONS}

In all techniques, the cosmetic outcome. improved over time until the $63^{\text {rd }}$ po.d., but afterwards, the improvement was minimal.

From the $9^{\text {th }}$ po.d onwards, glue application had the worst cosmetic outcome compared to the other techniques

The use of intradermal suture pattern with clips had the best cosmetic outcome until the $180^{\text {th }}$ po.d, but afterwards, the intradermal suture pattern with burying of the knot seemed to be better.

The first postoperative day the use of staples provoked the largest skin thickening at the wound area. However, this changed after the removal of staples and from the $12^{\text {th }}$ po.d, onwards this technique showed the less intense skin thickening.

Tissue adhesive application provoked the largest skin thickening from the $10^{\text {th }}$ po.d. until the end of the experiment at the $365^{\text {th }}$ po.d.

In all techniques, skin thickening at the wound area decreased to less than $0.25 \mathrm{~mm}$ after the $60^{\text {th }}$ po.d.

In all techniques, scar width decreased over time.

Scar width with tissue adhesive application was larger than that of the other techniques from the $9^{\text {th }}$ po.d. onwards, and was significantly wider than that of the intradermal suture patterns on the $365^{\text {th }}$ po.d.

More cases of mild inflammation were observed with the intradermal suture pattern with burying of the knot.

Wound area pigmentation was observed with all techniques from the $28^{\text {th }}$ until the $365^{\text {th }}$ po.d., however with the intradermal suture patterns was less intense and lasted a shorter period of time.

Cross scaring formations were observed in all scars with staples until the $16^{\text {th }}$ po.d., in $5 / 10$ on the $35^{\text {th }}$ po.d. and in $1 / 10$ scars on the $70^{\text {th }}$ po.d., to fade away afterwards.

Total clinical evaluation was constantly better with intradermal suture patters compared with the other two techniques. The intradermal suture pattern with burying of the knot was better than all the others at the end of the experimental period. 
High-frequency ultrasonography can be used to evaluate first intention healing of the canine skin. Ultrasonographic findings correlated very well with the clinical and histological findings and depicted the real ultrastructure of the skin during first intention healing.

On the outer surface of the thighs, ultrasonographically recorded wound area thickening was not even, but larger at the upper and lower section of the wound.

Both clinical and ultrasonographical evaluation showed that wound area thickening had the largest value 24 hours postoperatively, decreased substantially until the $14^{\text {th }}$ po.d., and then showed slight decrease until the $60^{\text {th }}$ po.d., reaching the normal values.

No differences were observed between the techniques in the ultrasonographically evaluated wound area.

No differences between the techniques were observed concerning inflammation, angeiogenesis, fibroblast presence and collagen deposition in any time period.

The thickness of epidermis at wound area was much larger with glue compared to the other techniques until the $28^{\text {th }}$ po.d., but there was no difference afterwards.

The total histological score was worse with glue in all time periods; however, this difference was not statistically significant.

Histological examination showed that, in spite of care in application, small quantities of glue penetrated the wound, initiating inflammation in the dermis of two animals.

Total evaluation of each technique (that includes cosmetic, clinical, ultrasonographical and histological scores) revealed that the intradermal suture patterns had the best score throughout the experiment period, with the intradermal suture pattern with burying of the knots being slightly better than the intradermal with clips, but without any statistically significant difference. Furthermore, tissue adhesive application had constantly the worse score.

Finally, when the least visible scar is the goal, intradermal suture pattern with burying of the knot should be used.

When the time required for skin closure is an important factor, or when the length of the skin to be sutured is considerably long, stapling should be considered the first choice. 


\section{ABSTRACT}

Most surgical wounds are typically treated by first intention healing with the use of sutures; however alternative closure techniques or materials, such as tissue adhesives and skin staples have also been used. Sutures enable meticulous closure and staples and tissue adhesives are credited for faster closure. However, up to now, the assessment of wound healing process has been mainly based on clinical evaluation of the wound, on the frequency of wound complications and on the cosmetic appearance of the wound. More detailed information on the status of the wound has relied on invasive skin biopsy, providing tissue samples that yield objective quantitative data relevant to the healing process, but this inevitably produces further damage. Cutaneous ultrasonography is a novel technology for skin wound assessment. The development of larger ultrasonic frequencies (above $20 \mathrm{MHz}$ ) and the introduction of "ultrasound biomicroscopy" in diagnostic imaging enhanced qualitative assessment of skin composition, monitoring and evaluation of skin function in various pathological conditions, and enabled assessment of small open skin wounds which heal by second intention. By measuring the area of the skin wound, the process and progression of open wound healing can be assessed without interfering with healing. However, up to date, ultrasonography has not been used as a means of studying the healing process of wounds or surgical incisions closed by different methods and materials neither in humans nor in other animal species.

The aim of the present study was to compare the efficacy of various techniques used for the closure of skin incisions in dogs via ultrasonographic (50 MHz), cosmetic, clinical and histological evaluation, to describe the ultrasonographic appearance of the healing skin, and to compare it with clinical and histological measurements. Whether the findings, obtained when very high frequencies are used, actually represent a valid adjunct to clinical and histological assessment of the healing process of the skin was also investigated. Furthermore, the difficulty and the time required to close each wound was also estimated. The following techniques and materials for wound closure were compared: (a) Skin staples, (b) n-butyl cyanoacrylate tissue glue, (c) Continuous intradermal suture pattern (with burying of the knot) by using 4-0 poliglecaprone 25 and (d) Continuous intradermal suture pattern by using polypropylene 4-0 with attachment clips (without burying the knot).

Ten healthy purpose-bred laboratory Beagles, 1-5 years old, were included in the study. Two skin incisions, $12 \mathrm{~cm}$ long, were made at the lateral surface of each thigh, which were parallel to the long axis of the femur, and $7 \mathrm{~cm}$ apart from each other. Afterwards, in all incisions, the subcutaneous tissue was closed primarily with continuous subdermal suture pattern by using 3-0 polyglactin 910 (Vicryl, Ethicon Inc, Somerville, NJ). Secondary, the skin of each incision was closed with a different technique. Skin staples (Proximate, Ethicon Inc, Somerville, NJ) [staples], n-butyl cyanoacrylate tissue glue (Vetbond, 3M) [glue], continuous intradermal suture pattern (with burying of the knot) by using 4-0 poliglecaprone 25 (Monocryl, Ethicon, Inc, Somerville, NJ) [intradermal B] and continuous intradermal suture pattern with clips 
(without burying the knot) by using 4-0 polypropylene (Securex, B Braun Aesculap AG \& Co KG, Tuttlingen, Germany) [intradermal C], were used. The time required to perform each technique was recorded.

Immediately after surgery and until the 10th postoperative day (po.d.), Elisabeth collars were placed on the dogs and bandages were applied to the thighs in order to protect the wound site. On the 10th po.d., the staples and polypropylene suture material with clips were removed.

Evaluation of wound healing of skin incisions

Cosmetic appearance was blindly evaluated by two experienced surgeons on a 1-10 visual analogue scale (1: excellent cosmetic result, 10: bad cosmetic result), by assessing the photographs taken on the 7th, 14th, 28th, 63rd, 91st, 120th , 150th, 180th, 210th, 240th, 270th, 300th, 330th and 360th po.d. The scores from the two assessors were averaged to generate the total cosmetic appearance score for each wound. The higher the total score, the poorer the wound cosmetic appearance was.

Clinical evaluation was performed immediately after surgical skin closure (Day 0), every day until the 10th po.d., on the 12th, 14th, 16th, 18th, 21st, 24th and 28th po.d., once a week until the end of the 3rd postoperative month, and once a month until the end of the experiment. The following parameters were evaluated: oedema of the skin at the incision site, erythema, scar width, abscessation, exudation, comedones, scar hyperpigmentation, staples loss, wound dehiscence, and cross scaring formation.

B-mode real-time ultrasonographic examination of the skin was performed by the use of a real-time ultrasound machine. The ultrasound unit (Longport Digital Scanner [LDS1], Longport International Ltd., Silchester, United Kingdom) was fitted with a 50 $\mathrm{MHz}$ probe. Four transverse images (a, b, c, d) were taken from each wound. Wounds were examined daily until the 10th po.d., on the 12th, 14th, 16th, 18th, 21st, 24th and 28th po.d., once a week until the end of the 3rd postoperative month, and once a month until the end of the experiment. Furthermore, a scan was performed in the area where skin punch biopsy would be performed, just a few minutes before the biopsy. The digitized scans were visualized using a color palette (rainbow). Images were compressed laterally to facilitate viewing of the wound area. The wound area calculations were performed by using the computer software.

Histological evaluation of the healing process was performed on the 7th, 14th, 28th, 180th and 365th po.d. The following parameters were evaluated: necrosis, epithelial gap, oedema, inflammation, the presence of suture, tissue reaction around the suture, epidermal thickness, scar width, the presence of fibroblasts, collagen production, and angiogenesis.

In order to find a representative score for the cosmetic, clinical, ultrasonographical and histological evaluation, at each time period, separate scores of the parameters of each category were added.

Furthermore, in order to compare the techniques over time, the total representative score of each technique was evaluated, on the 7th, 14th, 28th, 180th and 365th po.d, by adding each time the cosmetic, clinical, ultrasonographical and histological scores. 
In general, skin healed normally without any major complications. The overall conclusions or this study are:

With all techniques, the cosmetic outcome improved over time until the 63rd po.d., but afterwards the improvement was minimal.

From the 9th po.d onwards, glue application had the worst cosmetic outcome compared to the other techniques.

The use of intradermal suture pattern with clips had the best cosmetic outcome until the 180th po.d, but afterwards the intradermal suture pattern with burying of the knot seemed to be much better.

Staples induced the largest skin thickening at the wound area at the first postoperative days. However, this changed after their removal, and from the 12th po.d. onwards this technique showed the less intense skin thickening.

Tissue adhesive application induced the largest skin thickening from the 10th po.d. until the end of the experiment on the 365th po.d.

Skin thickening at the wound area decreased to less than $0.25 \mathrm{~mm}$ after the 60th po.d. with all techniques,

Scar width decreased over time with all techniques.

Scar width was larger with tissue adhesive than with the other techniques from the 9th po.d. onwards, and was significantly larger than with the intradermal suture patterns on the 365th po.d.

More cases of mild inflammation were observed with the intradermal suture pattern with burying of the knot.

Wound area hyperpigmentation was observed with all techniques from the 28th until the 365th po.d., however, with the intradermal suture patterns hyperpigmentation was less intense and lasted a shorter period of time.

Cross scaring formations were observed in all scars with staples until the 16th po.d., in $5 / 10$ scars on the 35 th po.d. and in $1 / 10$ scars on the 70 th po.d., to fade away afterwards.

The total clinical evaluation was constantly better with intradermal suture patterns compared with the other two techniques. The intradermal suture pattern with burying of the knot was better than all the others at the end of the experimental period.

High-frequency ultrasonography can be used to evaluate first intention healing of the canine skin. Ultrasonographic findings correlated very well with the clinical and histological findings and depicted the real ultrastructure of the skin during first intention healing.

On the outer surface of the thighs, ultrasonography revealed that wound area thickening was uneven, being larger at the upper and lower section of the wound.

Both clinical and ultrasonographical evaluation showed that wound area thickening had the largest value 24 hours postoperatively, decreased substantially until the 14th po.d., and then showed slight decrease until the 60th po.d., reaching the normal values. No differences were observed between the techniques in the ultrasonographically evaluated wound area.

No differences between the techniques were observed concerning inflammation, angiogenesis, fibroblast presence and collagen deposition in any time period. 
The thickness of epidermis at wound area was much larger with glue compared to the other techniques until the 28th po.d., but there was no difference onwards.

The total histological score was worse with glue in all time periods; however, this difference was not statistically significant.

Histological examination showed that, in spite of care in application, small quantities of glue penetrated the wound, initiating inflammation in the dermis of two animals.

The total evaluation of each technique (that includes cosmetic, clinical, ultrasonographical and histological scores) revealed that the intradermal suture patterns had the best score throughout the experiment period, with the intradermal suture pattern with burying of the knots being slightly better than the intradermal with clips, but without any statistically significant difference. Furthermore, tissue adhesive application had constantly the worse score.

Finally, when the least visible scar is the goal, intradermal suture pattern with burying of the knot should be used. However, when the time required for skin closure is an important factor, or when the length of the skin to be sutured is considerably long, stapling should be considered the first choice. 


\section{REFERENCES}

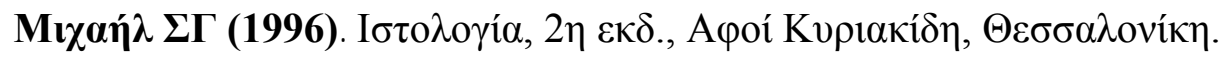

Aksoy M, Turnadere E, Ayalp K, Kayabali M, Ertugrul B, Bilgic L. (2006). Cyanoacrylate for wound closure in prosthetic vascular graft surgery to prevent infections through contamination. Surg Today 36:52-56.

Adams B, Anwar J, Wrone DA, Alam M (2003). Techniques for cutaneous sutured closures: variants and indications. Semin Cutan Med Surg, 22: 306-316.

Alam M, Posten W, Martini MC, Wrone DA, Rademaker AW (2006). Aesthetic and functional efficacy of subcuticular running epidermal closures of the trunk and extremity: a rater-blinded randomized control trial. Arch Dermatol 142: 12721278.

Amiel GE, Sukhotnik I, Kawar B, Siplovich L (1999). Use of n-butyl-2 cyanoacrylate in elective surgical incisions_-Longterm outcomes. J Am Coll Surg; 189:21-25.

Amin M, Glynn F \&Timon C (2009). Randomized trial of tissue adhesive vs staples in thyroidectomy integrating patient satisfaction and Manchester score. Otolaryngology-Head and Neck Surgery 140, 703-708.

Anate M (1991). Skin closure of laparotomy wounds: absorbable subcuticular sutures vs non-absorbable interrupted sutures. West Afr J Med, 10: 150-157.

Andrade MG, Weissman R, Reis SR (2006). Tissue reaction and surface morphology of absorbable sutures after in vivo exposure. J Mater Sci: Mater Med, 17: 949-961.

Applebaum JS, Zalut T, Applebaum D (1993). The use of tissue adhesion for traumatic laceration repair in the emergency department. Ann Emerg Med; 22:1190-1192.

Arunachalam P, King PA \& Orford J (2003). A prospective comparison of tissue glue versus sutures for circumcision. Pediatr Surg Int 19: 18-19 DOI 10.1007/s00383-002-0893-1.

Aspres N, Egerton IB, Lim AC, Shumack SP (2003). Imaging the skin. Australasian Journal of Dermatology 44, 19-27.

Banks WJ (1993). Integumentary system. In: Applied Veterinary Histology, $3^{\text {rd }}$ edn. Mosby Year Book, St. Louis, pp 298-325. 
Basha SL, Rochon ML, Quiñones JN, Coassolo KM, Rust OA, Smulian JC (2010). Randomized controlled trial of wound complication rates of subcuticular suture vs staples for skin closure at cesarean delivery. Am J Obstet Gynecol; 203:285.e1-8.

Bhaskar SN, Frisch J, Cutright DE, Margetis P (1967). Effect of butylcyanoacrylate on the healing of extraction wounds. Oral Surg Oral Med Oral Pathol. 1967;24:604-616.

Bennett RG (1988). Selection of wound closure materials. J Am Acad Dermatol, 18: 619-637.

Bessonart MN, Macedo N \& Carmona C (2005). High resolution B-scan ultrasound of hypertrophic scars. Skin Research and Technology; 11: 185-188.

Bezwada RS, Jamiolkowski DD, Lee I-Y, Agarwal V, Persivale J, Trenka-Benthin S, Erneta M, Suryadevara J, Yang A, Liu S (1995). Monocryl suture, a new ultra-pliable absorbable monofilament suture. Biomaterials, 16: 1141-1148.

Bhatia SK (2010). Biomaterials for Clinical Applications. Springer, New York.

Bleve M, Capra P, Pavanetto F, \& Perugini P (2012). Ultrasound and 3D skin imaging: Methods to evaluate efficacy of striae distensae treatment. Hindawi Publishing Corporation Dermatology Research and Practice Volume, Article ID 673706.

Blondeel PNV, Murphy JW, Debrosse D, Nix III JC, Puls LE, Theodore N, Coulthard P (2004). Closure of long surgical incisions with a new formulation of 2-octylcyanoacrylate tissue adhesive versus commercially available methods. The American Journal of Surgery 188 307-313.

Bohling MW, Henderson RA, Swaim SF, Kincaid SA, Wright JC (2006). Comparison of the role of the subcutaneous tissues in cutaneous wound healing in the dog and cat. Veterinary Surgery, 35: 3-14.

Booth HW Jr (1998). Selecting suture materials for small animal surgery. Compend Contin Edu Pract Vet 20:156.

Boothe HW (2003). Suture materials, tissue adhesives, staplers, and ligating clips. In: Slatter Textbook of Small Animal Surgery, vol, 1 3rd ed, Saunders, Philadelphia, pp.235-244.

Bouer M, Chammas MC, Messina MCL, Souza de Oliveira IR, Cerri GG (2008). Clinical and ultras onographic correlation in localized cutaneous scleroderma. Radiol Bras; 41(2):87-91. 
Bozkurt MK \& Saydam L (2008). The use of cyanoacrylates for wound closure in head and neck surgery. Eur Arch Otorhinolaryngol 265:331-335.

Broughton II G, Janis JE, Attinger CE (2006). Wound Healing: An Overview Plast. Reconstr. Surg. 117: 1e-S.

Brown JK, Campbell BT, Drongowski RA, Alderman AK, Geiger JD, Teitelbaum DH, Quinn J, Coran AG, Hirschl RB (2009). A prospective, randomized comparison of skin adhesive and subcuticular suture for closure of pediatric hernia incisions: cost and cosmetic considerations. Journal of Pediatric Surgery 44, 14181422.

Bruns TB \& Worthington JM (2000). Using tissue adhesive for wound repair: a practical guide to Dermabond. Am Fam Physician;61:1383-8.

Bruns TB, Robinson BS, Smith RJ, Kile DL, Davis TP, Sullivan KM, Quinn JV (1998). A new tissue adhesive for laceration repair in Children. J Pediatr; 132:1067-70.

Bruns TB, Simon HK, McLario DJ (1996). Laceration repair using a tissue adhesive in a children's emergency department. Pediatrics; 98: 673-5.

Bucknall TE (1983). Factors influencing wound complications: a clinical and experimental study. Ann R Coll Surg Engl; 65:71-77.

Cagle PE, Dyson M, Gajewski B, Lukert B (2007). Can dermal thick measured by ultrasound biomicroscopy assist in determining osteoporosis risk? Skin Research and Technology; 13: 95-100.

Chen L, Dyson M, Rymer J, Bolton PA and Young SR (2001).The use of highfrequency diagnostic ultrasound to investigate the effect of hormone replacement therapy on skin thickness. Skin Research and Technology; 7: 95-97.

Chibbaro S \& Tacconi L (2009). Use of skin glue versus traditional wound closure methods in brain surgery: A prospective, randomized, controlled study. Journal of Clinical Neuroscience 16 535-539.

Chow A, MRCS, Marshall H, Zacharakis E, Paraskeva P, Purkayastha S (2010). Use of tissue glue for surgical incision closure: A systematic review and metaanalysis of randomized controlled trials. J Am Coll Surg Vol. 211, No. 1, July 2010, 114-126.

Clay FSH, Walsh CA, Walsh SR (2011). Staples vs subcuticular sutures for skin closure at cesarean delivery: a metaanalysis of randomized controlled trials. American Journal of Obstetrics \& Gynecology. 
Coover HN, Joyner FB, Sheere NH (1959). Chemistry and performance of cyanoacrylate adhesive. Journal of the Society of Plastic Surgery of England; 1:56.

Coulthard P, Esposito M, Worthington HV, van der Elst M, van Waes OJF, Darcey J (2002). Tissue adhesives for closure of surgical incisions. Cochrane Database of Systematic Reviews, Issue 3. Art. No.: CD004287. DOI: 10.1002/14651858.CD004287.pub2.

Court MH, Bellenger CR (1989). Comparison of adhesive polyurethane membrane and polypropylene sutures for closure of skin incisions in cats. Vet Surg, 18: 211-215.

Crikelair GF (1958). Skin suture marks. Am J Surg, 96: 631-639.

Crisan D, Crisan M, Moldovan M, Lupsor M, Badea R(2012). Ultrasonographic assessment of the cutaneous changes induced by topical flavonoid therapy. Clinical, Cosmetic and Investigational Dermatology: 5 7-13.

Cromi A, Ghezzi F, Gottardi A, Cherubino M, Uccella S, Valdatta L (2010). Cosmetic outcomes of various skin closure methods following cesarean delivery: a randomized trial. Am J Obstet Gynecol; 203:36.e1-8.

De Graaf IM, K., Rengerink K.O., Wiersma I.C., Donker M.E., Mol B.W., E. Pajkrt E (2012). Techniques for wound closure at caesarean section: a randomized clinical trial. European Journal of Obstetrics \& Gynecology and Reproductive Biology 165, 47-52.

De la Cruz KI \& Cohn WE (2011). Stitches, glues, and staples. In: Pumps and Pipes: Proceedings of the Annual Conference. 105 DOI 10.1007/978-1-4419-6012-2_10, (C) Springer Science+Business Media.

Diana A, Guglielmini C, Fracassi F, Pietra M, Balletti E, Cipone M (2008). Use of high-frequency ultrasonography for evaluation of skin thickness in relation to hydration status and fluid distribution at various cutaneous sites in dogs. Am J Vet Res; 69:1148-1152.

Diridollou S, Black D, Lagarde JM, Gall Y, Berson M, Vabre V, Patat F, Vaillant L (2000). Sex- and site-dependent variations in the thickness and mechanical properties of human skin in vivo. International Science, 22 421-435.

Doshi SJ (1992). A complete review of wound closure. Massachusetts Institute of Technology. 
Dresner HS \& Hilger PA (2009). Comparison of incision closures with subcuticular and percutaneous staples. Arch Facial Plast Surg; 11(5):320-326.

Dunn DL, Phillips J (2004). Other surgical product in: ETHICON Wound closure manual.pp.75-77, (c) ETHICON, INC. 2004 Trademark ETHICON, INC., P.O. Box 151, Somerville, NJ 08876-0151.

Dyson M, Moodley S, Verjee L, Verling W, Weinman J, Wilson P (2003). Wound healing assessment using $20 \mathrm{MHz}$ ultrasound and photography. Skin Research and Technology; 9:116-121.

Edlich RF, Rodeheaver GT, Thacker JG, Lin KY, Drake DB, Mason SS, BS, Wack CA, Chase ME, Tribble C, Long III WB, \& Vissers RJ (2010). Revolutionary advances in the management of traumatic wounds in the emergency department during the last 40 years: Part II. The Journal of Emergency Medicine, Vol. 38, No. 2, pp. 201-207.

Eggers MD, Fang L, Lionberger DR(2011). A comparison of wound closure techniques for total knee arthroplasty. The Journal of Arthroplasty 26(8):1251-8.

El Gammal S, El Gammal C, Kaspar K, Pieck C, Altmeyer P, Vogt M, \& Ermert H (1999). Sonography of the skin at $100 \mathrm{MHz}$ enables in vivo visualization of stratum corneum and viable epidermis in palmar skin and psoriatic plaques. Journal of Investigative Dermatology 113:821-829.

Elemen L, Seyidov TH \& Tugay M (2011). The advantages of cyanoacrylate wound closure in circumcision. Pediatr Surg Int 27:879-883 DOI 10.1007/s00383-0102741-z.

Faria MCF, de Almeida FM, Serrao ML, de Oliveira Almeida NK, Labarthe N (2005). Use of cyanoacrylate in skin closure for ovariohysterectomy in a population control programme. Journal of Feline Medicine and Surgery (2005) 7, 71-75doi:10.1016/j.jfms.2004.05.003.

Farion KJ, Russell KF, OsmondMH, Hartling L, Klassen TP, Durec T, Vandermeer B (2001). Tissue adhesives for traumatic lacerations in children and adults. Cochrane Database of Systematic Reviews, Issue 4. DOI: 10.1002/14651858.CD003326.

Fornage BD, McGavran MH, Duvic M, Waldron CA (1993). Imaging of the Skin with 20-MHz US. Radiology; 189:69-76.

Foster FS, Pavlin CJ, Harasiewicz KA, Christopher DA, Turnbull DH (2000). Advances in ultrasound biomicroscopy. Ultrasound in Med. \& Biol. 26 (1) 1-27. 
Friend E (2009). Complications of wound healing. In: BSAVA Manual of Canine and Feline Wound Management and Reconstruction, $2^{\text {nd }}$ ed., John Williams and Alison Moores, pp. 254-270.

Gaertner I, Burkhardt T, Beinder E (2008). Scar appearance of different skin and subcutaneous tissue closure techniques in caesarean section: A randomized study. European Journal of Obstetrics \& Gynecology and Reproductive Biology 138 2933.

Gatt D, Quick CRG, Owen-Smith MS (1982). Staples for wound closure: a controlled trial. Annals of the Royal College of Surgeons of England vol. 67, 318320.

Gillette RL, Swaim SF, Sartin EA, Bradley DM, Coolman SL (2001). Effects of a bioactive glass on healing of closed skin wounds in dogs. Am J Vet Res, 62: 1149-1153.

Gniadecka M. (2001). Effects of aging on dermal echogenicity. Skin Res Technol. 7:204-207.

Gouletsou PG (2014). Continuous intradermal suture pattern in the dog. comparison with the simple interrupted suture pattern and evaluation of the effect of suture material and size. Ph.D. thesis.

Graham DA, Jeffery JA, Bain UD, Davies P, Bentley G (2000). Staple vs. subcuticular vicryl skin closure in knee replacement surgery: a spectrophotographic assessment of wound characteristics. The Knee 7 239-243.

Greenberg JA, Clark RM (2009). Advances in suture material for obstetric and gynecologic surgery. Reviews in Obstetrics \& Gynecology 2(3):146-58.

Greene D, Koch RJ, and Goode R L (1999). Efficacy of octyl-2-cyanoacrylate tissue glue in blepharoplasty. A prospective controlled study of wound-healing characteristics. Arch Facial Plast Surg 1999. 1:292-296.

Greenhill GA, Barry O’Regan B (2009). Incidence of hypertrophic and keloid scars after N-butyl 2-cyanoacrylate tissue adhesive had been used to close parotidectomy wounds: A prospective study of 100 consecutive patients. British Journal of Oral and Maxillofacial Surgery 47, 290-293.

Gregory CR (1999). Wound healing and influencing factors. In: Manual of Canine and Feline Wound Management and Reconstruction. Fowler D, Williams JM (eds), British Small Animal Veterinary Association, Cheltenham, pp. 13-23. 
Hedlund CS (2007). Surgery of the integumentary system. In: Small Animal Surgery, $3^{\text {rd }}$ ed., Mosby Elsevier, St. Louis, pp. 159-251.

Holger JS, Wandersee SC \& Hale DB (2004). Cosmetic outcomes of facial lacerations repaired with tissue-adhesive, absorbable, and nonabsorbable Sutures. Am J Emerg Med; 22:254- 257.

Hopkinson GB, Bullen BR, (1982). Removable subcuticular skin suture in acute appendicitis: a prospective comparative clinical trial. Br Med J (Clin Res Ed) 284 (6319): 869.

Hosgood G (2003).Wound repair and specific tissue response to injury. In: Slatter Textbook of Small Animal Surgery, vol, 1 3rd ed, Saunders, Philadelphia, pp.67.

Hosgood G (2006). Stages of wound healing and their clinical relevance. Vet Clin North Am Small Anim Pract, 36: 667-685.

Hosgood G (2009). The biology of wound healing. In: BSAVA Manual of Canine and Feline Wound Management and Reconstruction, $2^{\text {nd }}$ ed., John Williams and Alison Moores, pp.1-14.

Hovaghimian DG, Abou Sedira KA, Farag MY(2015). N-butyl-2-cyanoacrylate tissue adhesive versus subcuticular skin closure in external dacryocystorhinostomy. Delta J Ophthalmol 16:97-102

Huang YP, Zheng YP, Leung SF \& Mak AFT (2007). Reliability of measurement of skin ultrasonic properties in vivo: a potential technique for assessing irradiated skin. Skin Research and Technology; 13:55-61.

Hunt TK, Hopf H, Hussain Z (2000). Physiology of wound healing. Adv Skin Wound Care, 13: 6-11.

Iavazzo C, Gkegkes ID, Vouloumanoy EK, Mamais I, Peppas G, Falagas ME (2011). Sutures versus staples for the management of surgical wounds: A metaanalysis of randomized controlled trials. The American Surgeon. 77(9):1206-21.

Johnson RG, Cohn WE, Thurer RL, McCarthy JR, Sirois CA, \& Weintraub RM (1997). Cutaneous closure after cardiac operations. A controlled, randomized, prospective comparison of intradermal versus staple closures. Annals of Surgery 226(5)606-612.

Johnston DE (1990). Wound healing in skin. Veterinary Clinics of North America Small Animal Practice, 20: 1-25. 
Kanegaye JT, Vance CW, Chan L, Schonfeld N (1997). Comparison of skin stapling devices and standard sutures for pediatric scalp lacerations: A randomized study of cost and time benefits. J Pediatr; 130:808-13.

Khan R. J. K, Fick D, Yao F, Tang K, Hurworth M, Nivbrant B, Wood D. (2006). A comparison of three methods of wound closure following arthroplasty. J Bone Joint Surg Br 88(2)238-42.

Kirpensteijn J, Maarschalkerweerd RJ, Koeman JP, Kooistra HS, Van Sluijs FJ (1997). Comparison of two suture materials for intradermal skin closure in dogs. Vet Q, 19: 20-22.

Knott PD, MD, Zins JE, Banbury J, Djohan R, Yetman RJ, Papay F (2007). A comparison of dermabond tissue adhesive and sutures in the primary repair of the congenital cleft lip. Ann Plast Surg; 58: 121-125.

Konstantinov I (2004). Circular vascular stapling in coronary surgery. Ann Thorac Surg; 78:369 -73.

Kowalsky MS, Dellenbaugh SG, Erlichman DB, et al (2008). Evaluation of suture abrasion against rotator cuff tendon and proximal humerus bone arthroscopy; 24:329-334.

Leaper DJ, Benson CE (1985). Subcuticular skin closure after inguinal surgery. J R Coll Surg Edinb, 30: 234-236.

Lee HK, Seo YK, Baek JH \& Koh JS (2008). Comparison between ultrasonography (Dermascan C version 3) and transparency profilometry (Skin Visiometer SV600). Skin Research and Technology; 14: 8-12.

Li J, Chen J, Kirsner R (2007). Pathophysiology of acute wound healing. Clinics in Dermatology 25, 9-18.

Liang HD \& Blomley MJK (2003). The role of ultrasound in molecular imaging. The British Journal of Radiology, 76, suppl 2, S140-S150.

Lins RDAU, Gomes RCB, dos Santos KSA, da Silva RV, da Silva RTM, Ramos IA (2012). Use of cyanoacrylate in the coaptation of edges of surgical wounds. An Bras Dermatol; 87(6):871-6.

Livesey C, Wylde V, Descamps S, Estela C.M, Bannister G.C, Learmonth I.D, Blom A.W (2009). Skin Closure after Total Hip Replacement. A Randomised Controlled Trial of Skin Adhesive versus Surgical Staples. J Bone Joint Surg Br 91(2):725-9. 
MacPhail C (2002). Gastrointestinal obstruction. Clinical Techniques in Small Animal Practice, 17(4)178-183.

MacPhail CM. (2013). Biomaterials, Suturing, and Hemostasis. In: Small Animal Surgery, $4^{\text {th }}$ ed., Fossum TW (ed.), Mosby, St. Louis, pp. 64-83.

Magee WP, Ajkay N, Githae B, Rosenblum RS (2003). Use of octyl-2cyanoacrylate in cleft lip repair. Ann Plast Surg; 50:1-5.

Manning TO, Monteiro-Riviere NA, Bristol DG, Riviere JE (1991). Cutaneous laser-Doppler velocimetry in nine animal species. Am J Vet Res ;52:1960-1964.

Mantis P, Lloyd DH, Pfeiffer D, Stevens K, Auxilia S, Noli C, Abramo F, Miolo A (2007). Veterinary wounds: Assessment of the effect of an aliamide-containing topical gel by evaluation of the reduction of wound volume measurement by high resolution ultrasound biomicroscopy. Wounds 19(5)113-119.

Mantis P, Pfeiffer D, Stevens K, Auxilia S, Noli C, Abramo F (2005). Highresolution ultrasonography of experimentally induced full-thickness canine skin wounds: Efficacy in imaging canine skin and comparison of 2 methods of measuring wound site. Wounds; 17(5): 107-113.

Mantis P, Tontis D, Church D, Lloyd D, Stevens K, Balomenos D, Pagona G. Gouletsou PG, Gianoulopoulos G, Doukas D, Galatos AD, Saridomichelakis (2014). High-frequency ultrasound biomicroscopy of the normal canine haired skin. Veterinary Dermatology 25(3):176-81.

Martin P (1997). Wound healing--aiming for perfect skin regeneration. Science, 276: 75-81.

Matsumoto K, Robb E, Warden G, Nordlund J (1996). Hyperpigmentation of human skin grafted on to athymic nude mice: immunohistochemical study. $\mathrm{Br} \mathrm{J}$ Dermatol, 135: 412-418.

Mattick A (2002). Use of tissue adhesives in the management of paediatric Lacerations. Emerg Med J; 19:382-385.

Maw JL, Quinn JV, Wells GA (1997). A prospective comparison of octyl cyanoacrylate tissue adhesive and sutures for the closure of head and neck incisions. J Otolaryngol; 26:26-30.

McGeady TA, Quinn PJ, FitzPatrick ES, Ryan MT, Cahalan S (2006b). Integumentary system. In: Veterinary Embryology. Blackwell, Oxford, pp 313330. 
Medina dos Santos LR, Freitas CAF, Hojaji FC (1995). Prospective study using skin staplers in head and neck surgery. Am J Surg; 170:451-452.

Miller W, Griffin C, Campell K, (2013a). Structure and function of the skin. In: Muller \& Kirk’s Small Animal Dermatology, $7^{\text {th }}$ ed., Miller WHJr, Griffin CE G, Campell K (eds), Elsevier, St. Louis, pp. 1-56.

Miller W, Griffin C, Campell K, (2013b). Pigmentary abnormalities. In: Muller \& Kirk’s Small Animal Dermatology, $7^{\text {th }}$ ed., Miller WHJr, Griffin CE G, Campell K (eds), Elsevier, St. Louis, pp. 618-629.

Mizrahi B, Stefanescu CF, Yang C, Lawlor MW, Ko D, Langer R, Kohane DS (2011). Elasticity and safety of alkoxyethyl cyanoacrylate tissue adhesives. Acta Biomaterialia 7(8)3150-3157.

Mobley S.R, Hilinski J, Toriumi D.M (2002). Surgical tissue adhesives. Facial Plast Surgery Clin N Am 10 147-154.

Moghimi S, Baygi MHM, Torkaman G (2011). Automatic evaluation of pressure sore status by combining information obtained from high-frequency ultrasound and digital photography. Computers in Biology and Medicine 41, 427-434.

Molea G, Schonauer F, Bifulco G, D' Angelo D (2000). Comparative study on biocompatibility and absorption times of three absorbable monofilament suture materials (Polydioxanone, Poliglecaprone 25, Glycomer 631). Br J Plast Surg, 53: 137-141.

Mondini A, Bianchi L, Zagra L (2012). Wound closure and wound monitoring in total hip arthroplasty. An overview. Hip Int; 22 (Suppl 8): S15-S18.

Monteiro-Riviere NA, Stinson AW, Calhoun HL (1993). Integument. In: Textbook of Veterinary Histology, $4^{\text {th }}$ edn. H-Dieter Dellmann (ed). Lea \& Febiger, Philadelphia, pp 285-312.

Moore DC, Sellers MH, Archer KR, Schwartz HS, Holt GE (2013). Staples equal sutures for skin closure after soft tissue tumor resection. Clin Orthop Relat Res. 471(3): 899-904

Murphy M, Prendergast P, John Rice J (2004). Comparison of clips versus sutures in orthopaedic wound closure. Eur J Orthop Surg Traumatol 14: 16-18.

Nagpal BBM, Kumar MG, Nagi MGS, Singh WCP (2004). Sutureless closure of operative skin wounds. MJAFI; 60: 131-133. 
Newman JT, Morgan SJ, Resende GV, Williams AE, Hammerberg EM, Dayton MR (2011). Modality of wound closure after total knee replacement: are staples as safe as sutures? A retrospective study of 181 patients. Patient Safety in Surgery, 5:26.

Nitsch A, Pabyk A, Honig JF, Verheggen R, Merten HA (2005). Cellular, histomorphologic, and clinical characteristics of a new octyl-2-cyanoacrylate skin adhesive. Aesth. Plast. Surg. 29:53-58.

Nyland TG \& Mattoon JS (2002). Physical principles, instrumentation, and safety of diagnostic ultrasound In: Small Animal Diagnostic Ultrasound. Second Edition, Saunders, Philadelphia, pp.1-18.

Obermair A, Crandon A, Perrin L, Walsh T, Carrazo M, Nicklin J (2007). Randomized trial of skin closure after laparotomy for gynaecological surgery. ANZ Journal of Surgery, 77: 460-463.

O'Brien MG (1998). Principles of oncologic abdominal surgery. ClinicalTechiqques in Small Animal Practice 113(1)42-46.

Olsen LO, Takiwaki H \& Serup J (1995). High-frequency ultrasound characterization of normal skin. Skin thickness and echographic density of 22 anatomical sites. Skin Research and Technology 1: 74-80.

Ong J, Ho KS, Chew MH, Eu KW (2010). Prospective randomised study to evaluate the use of dermabond ProPen (2-octylcyanoacrylate) in the closure of abdominal wounds versus closure with skin staples in patients undergoing elective colectomy. Int J Colorectal Dis 25:899-905.

Orozco-Covarrubias ML, Ruiz-Maldonado R (1999). Surgical facial wounds: simple interrupted percutaneous suture versus running intradermal suture. Dermatol Surg, 25: 109-112.

Osmond MH (1999). Pediatric wound management: the role of tissue adhesives. Pediatric Emergency Care 15(2):137-40.

Othman I (2010). Skin glue improves outcome after excision and primary closure of sacrococcygeal pilonidal disease. Indian J Surg 72(6):470-474.

Papazoglou L G, Tsioli V, Papaioannou N, Georgiadis M, Savvas I, Prassinos N, Kouti V, Bikiaris D, Hadzigiannakis C, Zavros N (2010). Comparison of absorbable and nonabsorbable sutures for intradermal skin closure in cats. Can Vet J; 51:770-772. 
Parell GJ, Becker GD (2003). Comparison of absorbable with nonabsorbable sutures in closure of facial skin wounds. Arch Facial Plast Surg, 5(6) 488-90.

Pavletic MM (2010). Atlas of Small Animal Wound Management and Reconstructive Surgery. $3^{\text {rd }}$ ed. Wiley-Blackwell, Iowa.pp. 17-29.

Perron AD, Garcia JA, Hays EP (2000). The efficacy of cyanoacrylate-derived surgical adhesive for use in the repair of lacerations during competitive athletics. American Journal of Emergency Medicine; 18: 261-3.

Pesic Z, Buric N, Vuckovic I, Petrovic D, Krasic D, Cosic A, Djokic I (2011). Use of 2-octyl-cyanoacrylate in surgical closing of postparotidectomy salivary fistulas. Eur Arch Otorhinolaryngol 268:1691-1694.

Peter Lorenz H and Longaker MT (2008). Wounds: biology, pathology, and management. In: Surgery Basic Science and Clinical Evidence, 2nd ed. Springer New York, pp 191-208.

Pope ER (1993). Skin healing. In: Disease Mechanisms in Small Animal Surgery, $2^{\text {nd }}$ edn. Bojrab MJ (ed). Lea \& Febiger, Philadelphia, pp 151-155.

Postlethwait R, Willigan D, Ulin A (1975). Human tissue reaction to sutures. Ann Surg, 181: 144-150.

Pronio A, Di Filippo A, Narilli P, Caporillli D, Vestri A, Ciamberlano B, Pelle F, Montesani C (2011). Closure of cutaneous incision after thyroid surgery: a comparison between metal clips and cutaneous octyl-2-cyanoacrylate adhesive. A prospective randomized. Eur J Plast Surg 34: 103-110.

Quinn J, Wells G, Sutcliffe T, Jarmuske M (1997). A randomized trial comparing octylcyanoacrylate tissue adhesive and sutures in the management of lacerations. Journal of the American Medical Association; 277(19): 1527-30.

Quinn JV, Drzewiecki A, Li MM, Stiell IG, Sutcliffe T, Elmslie TJ, Wood WE (1993). A randomized, controlled trial comparing a tissue adhesive with suturing in the repair of pediatric facial lacerations. Ann Emerg Med; 22:1130-5.

Raišutis R, Jasiūnienė E, Jasaitienė D, Valiukevičienė S (2010). Investigation of human skin using pulse-echo ultrasonic technique: review and development. ISSN 1392-2114 Ultragarsas (Ultrasound) 65(1) 37-41

Ranaboldo C.J, Rowl Jones D.C. (1992). Closure of laparotomy wounds: skin staples versus sutures Br. J. Surg, 79:1172-1173.

Remedios A (1999). Complications of wound healing. In: BSAVA Manual of Canine and Feline Wound Management and Reconstruction. 
Ribeiro CM, Silva Júnior VA, Silva Neto JC, Vasconcelos BC (2005). Estudo clínico e histopatológico da reação tecidual às suturas interna e externa dos fios monofilamentares de nylon e poliglecaprone 25 em ratos. [Clinical and histopathological study of tissue reactivity to monofilament suture materials: nylon and poliglecaprone 25 in rats.] Acta Cir Bras, 20: 284-291.

Ridgway DM, Mahmood F, Moore L, Bramley D, Moore PJf (2007). A blinded, randomised, controlled trial of stapled versus tissue glue closure of neck surgery incisions. Ann R Coll Surg Engl 89: 242-246.

Rippon M.G, Springett K, Walmsley R, Patrick K, Millson S (1998). Ultrasound assessment of skin and wound tissue comparison with histology. Skin Research and Technology 4: 147-154.

Rivera AE \& Spencer JM (2007). Clinical aspects of full-thickness wound healing. Clinics in Dermatology 25, 39-48.

Robicsek F, Konstantinov I, Hult HI (2001). The father of the surgical stapler. Journal of Medical Biography; 9: 16-19.

Ronis ML, Hardwick JD, Fung R, Dellavecchia M (1984). Review of cyanoacrylate tissue glues with emphasis on their otorhinolaryngological applications. Laryngoscope. 94:210-13.

Rosenzweig LB, Abdelmalek M, Ho J, Hruza GJ (2010). Equal cosmetic outcomes with 5-0 poliglecaprone-25 versus 6-0 polypropylene for superficial closures. Dermatol Surg, 36: 1126-1129.

Rosin D, Rosenthal RJ, Kuriansky J, Brasesco O, Shabtai M, Ayalon A (2004). Closure of laparoscopic trocar site wounds with cyanoacrylate tissue glue: A simple technical solution. Journal of laparoendoscopic and advanced surgical techniques 11(3)157-159.

Roush JK (2003). Biomaterials and Surgical Implants in: Slatter Textbook of Small Animal Surgery, vol, 1 3rd ed, Philadelphia, Saunders, pp.141-148.

Rousseau JA, Girard K, Turcot-Lemay L, Thomas N (2009). A randomized study comparing skin closure in cesarean sections: staples vs subcuticular sutures. Am J Obstet Gynecol; 200:265.e1-265.e4.

Rudolph R, Klein L (1973). Healing processes in skin grafts. Surg Gynecol Obstet. 136(4):641-54. 
Runk A, Allen SW, Mahaffey EA (1999). Tissue reactivity to poliglecaprone 25 in the feline linea alba. Vet Surg, 28: 466-471.

Salthouse T (1983). Tissue response to sutures. In: Biomaterials in Reconstructive Surgery. Rubin R (ed.), Mosby, St. Louis, pp. 131-142.

Sanni A, Dunning J (2007). Staples or sutures for chest and leg wounds following cardiovascular surgery. Interactive CardioVascular and Thoracic Surgery 6:243246.

Satteson ES, Joseph A Molnar JA. Updated: Feb 13, 2015. Materials for Wound Closure. Elston DM ed. https://www.scribd.com/document/259621461/Materials-for-Wound-Closure

Satish L, Kathju S (2010). Cellular and molecular characteristics of scarless versus fibrotic wound healing. Dermatol Res Pract, Article ID 790234, 11 pages. doi:10.1155/2010/790234.

Saxena AK \& Willital GH (1999). Octylcyanoacrylate tissue adhesive in the repair of pediatric extremity lacerations. Am Surg. 65(5):470-2.

Scardino MS, Swaim SF, Sartin EA, Steiss JE, Spano JS, Hoffman CE, Coolman SL, Peppin BL (1998). Evaluation of treatment with a pulsed electromagnetic field on wound healing, clinicopathologic variables, and central nervous system activity of dogs. Am J Vet Res, 59: 1177-1181.

Schmid-Wendtner MH \& Burgdorf W (2005). Ultrasound Scanning in Dermatology. Arch Dermatol; 141:217-224.

Scott DW, Miller WHJr, Griffin CE (2001a). Structure and function of the skin. In: Muller \& Kirk's Small Animal Dermatology, $6^{\text {th }}$ ed. WB Saunders, Philadelphia, pp 1-70.

Shuster M (1989). Comparing skin staples to sutures in an emergency department. Can Fam Physician. 35: 505-509.

Singer AJ \&Thode HC (2004). A review of the literature on octylcyanoacrylate tissue adhesive The American Journal of Surgery 187, 238-248.

Singer AJ, Hollander JE, Valentine SM, Turque TW, McCuskey CF, Quinn JV (1998). Prospective, randomized, controlled trial of tissue adhesive (2octylcyanoacrylate) vs standard wound closure techniques for laceration repair. Acad. Emerg. Med. 5(2):94-99. 
Singer AJ, MD, Quinn JV, Clark RE, \& Hollander JE (2002). Closure of lacerations and incisions with octylcyanoacrylate: A multicenter randomized controlled trial. Surgery 131:270-276.

Singer AJ, Quinn JV, Hollander JE (2008). The cyanoacrylate topical skin adhesives. American Journal of Emergency Medicine 26: 490-496.

Smeac DD, Cassie AB, Chatterjee AK, Mehta S, Haworth JM (1988). Pain quantum and wound healing: a comparison of interrupted inversion PDS and standard nylon sutures in abdominal skin closure. Ann R Coll Surg Engl 70:339_ 342.

Smeak DD (1992). Buried continuous intradermal suture closure. Compend Contin Educ Pract Vet 14:907-919.

Smith TO (2010). Sutures versus staples for skin closure in orthopaedic surgery: meta-analysis. BMJ 340:c1199.

Song T, Wang Y, Li H, Wu D, Yin N (2013). Early cosmetic outcomes with the use of skin adhesives: Meta-analysis of randomized controlled trials. J Plast Reconstr Aesthet Surg. 66(2):292-4.

Stegmaier OC (1982). Use of skin stapler in dermatologic surgery. Am Acad Dermatol. 6(3):305-309.

Stockley I, Elson RA (1987). Skin closure using staples and nylon sutures: a comparison of results. Ann R Coll Surg Engl. 69(2):76-8.

Swaim SF, Henderson RA (1997). Small Animal Wound Management, $2^{\text {nd }}$ ed., Williams \& Wilkins, Baltimore.

Switzer EF, Dinsmore RC, North JH (2003). Subcuticular closure versus Dermabond. A prospective Randomized Trial. The American Surgeon pp. 434436 vol 69.

Sylvestre A, Wilson J, Hare J (2002). A comparison of 2 different suture patterns for skin closure of canine ovariohysterectomy. Can Vet J, 43: 699-702.

\section{Szymanska E, Nowicki A, Mlosek K, Litniewski J, Lewandowski M, Secomski W,} Tymkiewicz R (2000). Skin imaging with high frequency ultrasound preliminary Results. European Journal of Ultrasound 12, 9-16.

Teoh MK, Burd DAR, Bucknall TE (1987). Removal of skin staples in an emergency. Ann R Coll Surg Engl. 69(5): 222-224.

Terhune M (2012). (Medscape) 
Thomas WEG (2005). Sutures and staples. The Medicine Publishing Company Practical Procedures, 40-42.

Tobias KM (2007). Surgical stapling devices in veterinary medicine: A review. Veterinary Surgery; 36:341-349.

Toriumi DM, O'Grady K, Desai D, Bagal A (1998). Use of octyl-2-cyanoacrylate for skin closure in facial plastic surgery. Plast Reconstr Surg; 102:2209-2219.

Toriumi DM, Raslan WF, Friedman M, Tardy ME (1990). Histotoxicity of cyanoacrylate tissue adhesives. Arch Otolaryngol Head Neck Surg 116:546-50.

Toriumi DM, Raslan WF, Friedman M, Tardys ME Jr (1991). Variable histotoxicity of histoacryl when used in a subcutaneous site: an experimental study. Laryngoscope 101(4 Pt 1): 339-43.

Trott AT (1997). Cyanoacrylate tissue adhesives. An advance in wound care. JAMA; 277:1559-60.

Trout NJ (2003). Principles of plastic and reconstructive surgery. In: Slatter Textbook of Small Animal Surgery, vol, 1 3rd ed, Saunders, Philadelphia, pp. 274-291.

Tsioli V, Papazoglou LG, Papaioannou N, Psalla D, Savvas I, Pavlidis L, Karayannopoulpou (2015). Comparison of three skin stretching devices for closure of skin defects on the limbs of dogs. M. J Vet Sci. 16: 99-106.

Turnbull DH, Starkoski BG, Harasiewicz KA, Semple JL, From L, Guta AL, Sauder DN \& Fosters FS (1995). A 40-100 MHz B-scan ultrasound backscatter microscope for skin imaging. Ultrasound in Med. \& Biol. 21(1) 79-88.

United States Surgical Corporation History. http:/www.fundinguniverse.com/ company-histories/united-states-surgical-corporation-history/

Vaillant L (2004). High-Frequency ultrasound of the skin. In: Measuring the Skin. Springer-Verlag, Berlin, pp 204-214.

van den Ende ED, Vriens PW, Allema JH, Breslau PJ (2004). Adhesive bonds or percutaneous absorbable suture for closure of surgical wounds in children. Results of a prospective randomized trial. J Pediatr Surg. 39(8):1249-51.

van Heerden Johan (2005). Comparison of inflammatory response to polyglitone 6211 and poliglecaprone 25 in a rat model. SAMJ, 95: 972-974.

Van Winkle W, Hastings C, Barker E, Hines D, Nichols W (1975). Effect of suture materials on healing skin wounds. Surg Gynecol Obstet, 140: 7-12. 
Vasseur PB, Levy J, Dowd E, Eliot J (1988). Surgical wound infection rates in dogs and cat. Data from a teaching hospital. Vet Surg. Mar-Apr; 17(2):60-4.

Vipond MN, Higgins AF (1991). Subcuticular Prolene or PDS for skin closure? J R Coll Surg Edinb, 36: 97-99.

Wachter D, Brückel A, Stein M, Oertel MF, Christophis P, Böker DK (2010). 2Octyl-cyanoacrylate for wound closure in cervical and lumbar spinal surgery. Neurosurg Rev 33:483-489.

Waller JM \& Maibac HI (2005). Age and skin structure and function, a quantitative approach (I): blood flow, pH, thickness, and ultrasound echogenicity. Skin Research and Technology; 11: 221-235.

Webster RC, McCollough EG, Giandello PR, Smith RC (1985). Skin wound approximation with a new suture material. Arch Otolaryngol, 111: 517-519.

Winkler JT, Swaim SF, Sartin EA, Henderson RA, Welch JA (2002). The effect of a porcine-derived small intestinal submucosa product on wounds with exposed bone in dogs. Vet Surg, 31: 541-551.

Wollina U, Berger M \& Karte K (2001). Calculation of nail plate and nail matrix parameters by $20 \mathrm{MHz}$ ultrasound in healthy volunteers and patients with skin disease. Skin Research and Technology 7: 60-64.

Wortsman X, Gutierrez M, Saavedra T, Honeyman J (2011). The role of ultrasound in rheumatic skin and nail lesions: a multi-specialist approach. Clin Rheumatol 30:739-748.

Wysocki AB (1989). Surgical Wound Healing a Review for perioperative nurses. AORN JOURNAL 49(2) 502-518.

Zanna G, Fondevila D, Ferrer L, Espada Y (2012). Evaluation of ultrasonography for measurement of skin thickness in Shar-Peis; Am J Vet Res. 73(2):220-6. 
Institutional Repository - Library \& Information Centre - University of Thessaly 26/04/2023 13:56:30 EEST - 54.147.182.235 


\section{ADDENDUM la}


Institutional Repository - Library \& Information Centre - University of Thessaly 26/04/2023 13:56:30 EEST - 54.147.182.235 


\section{Beagle Bu1}
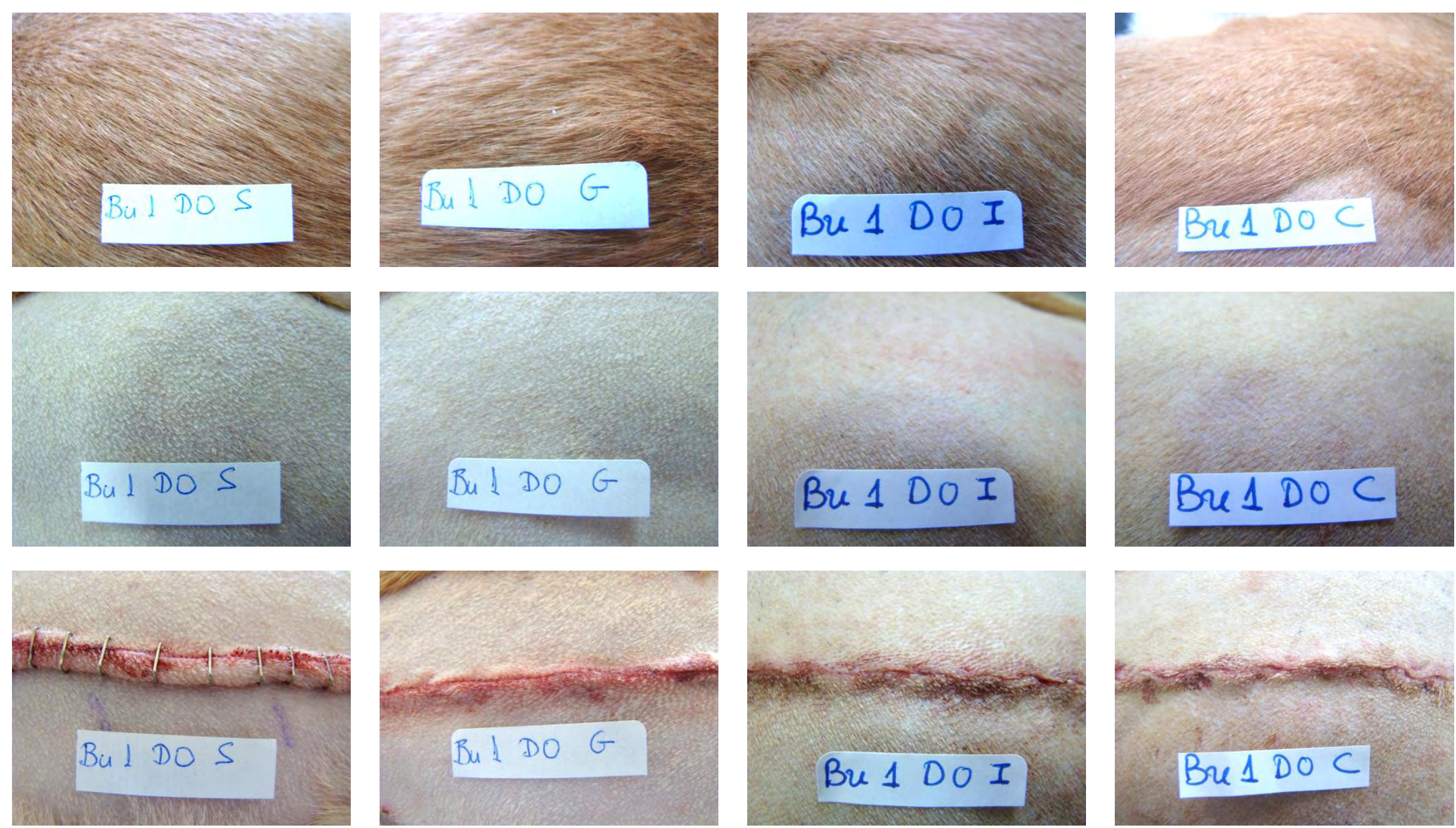


\section{Beagle Bu1}
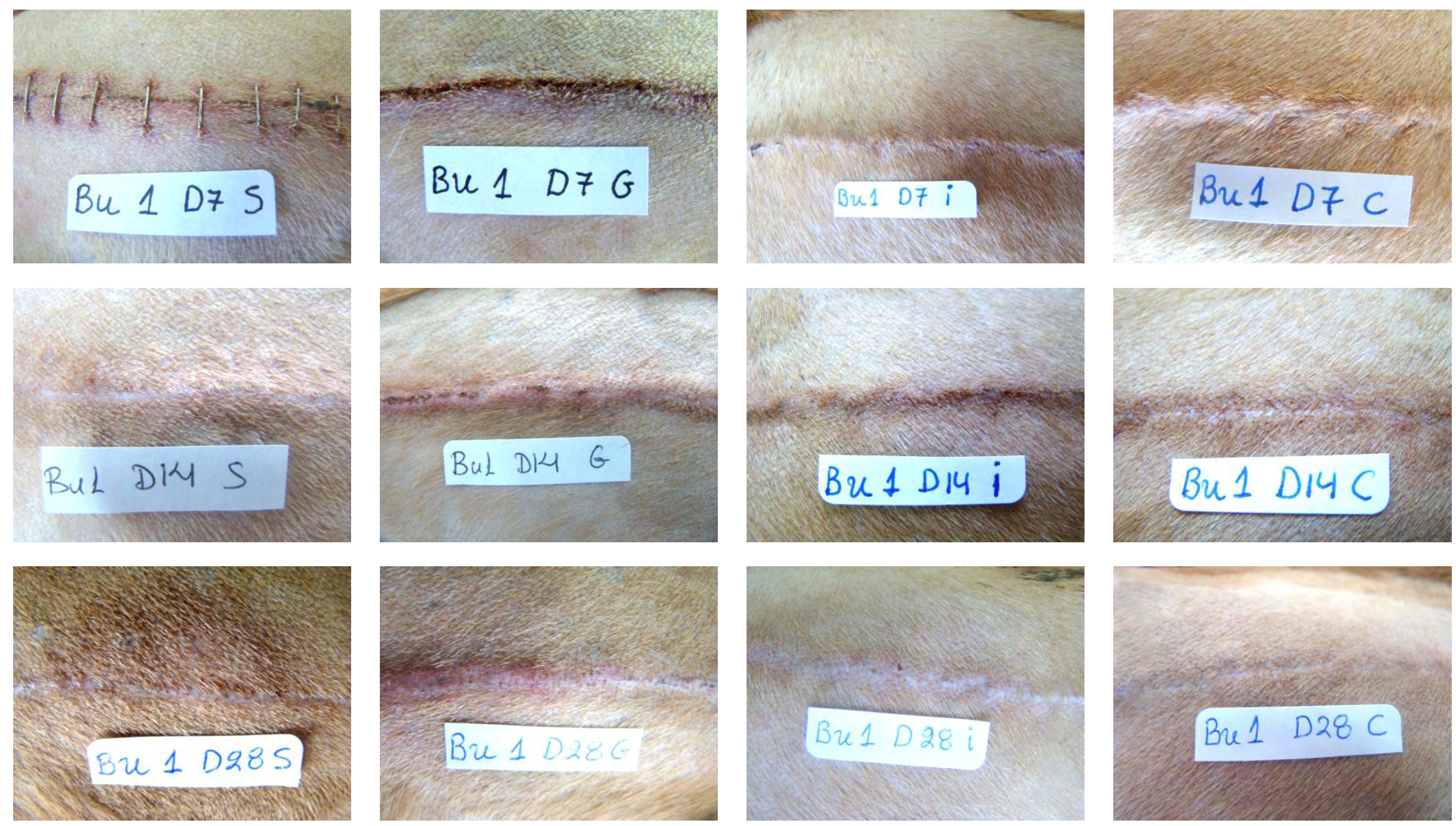


\section{Beagle Bu1}
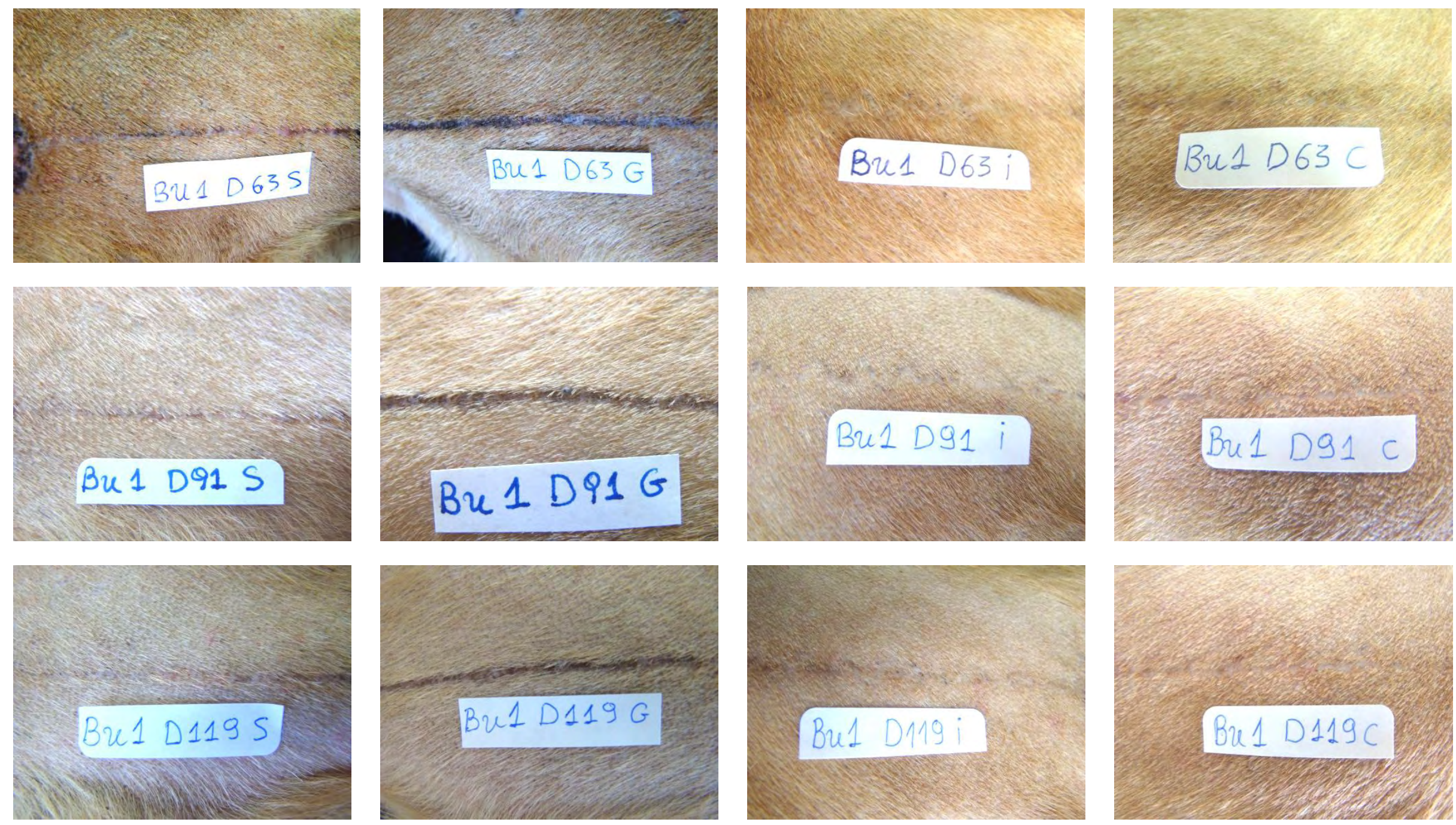


\section{Beagle Bu1}
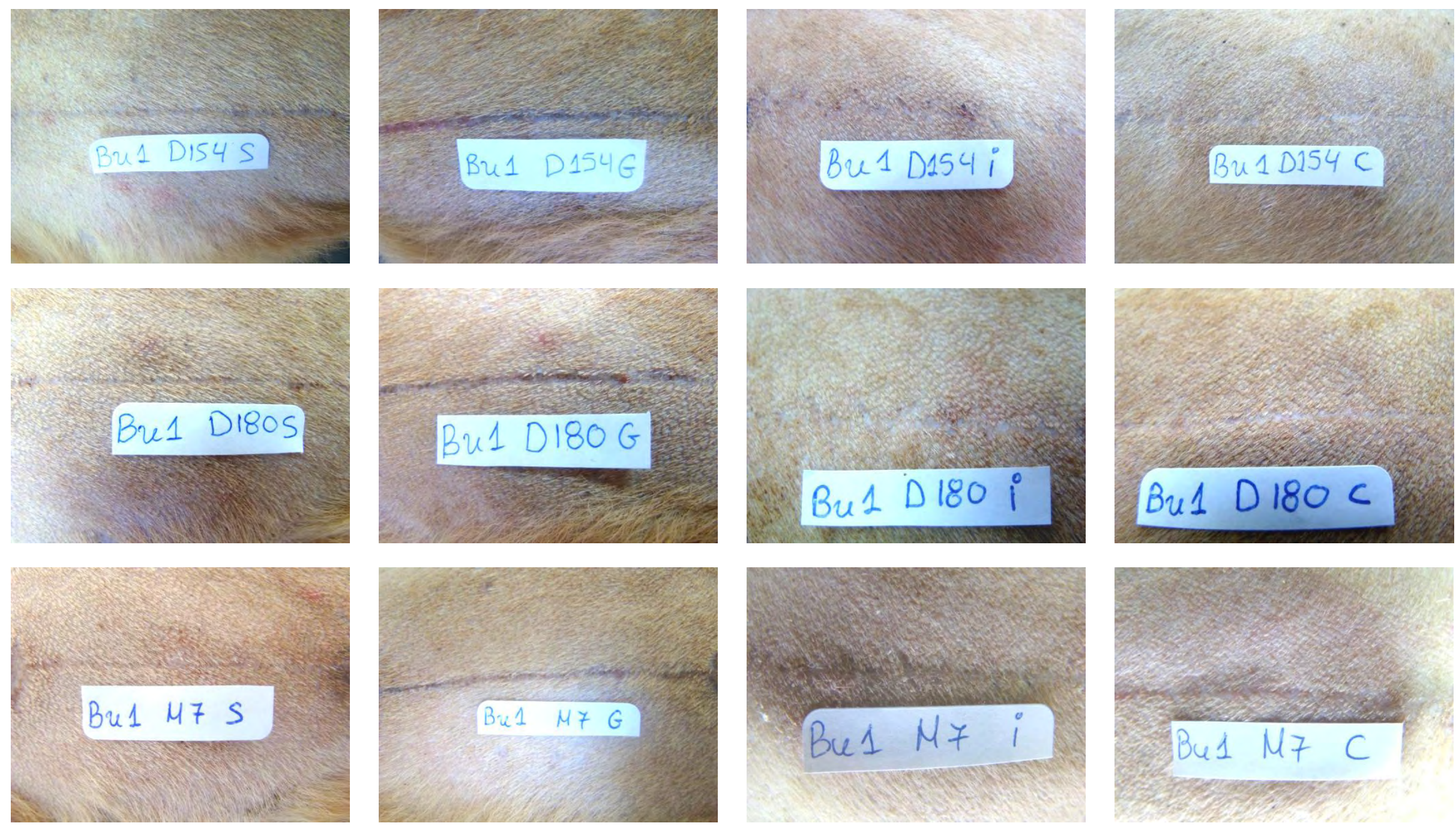


\section{Beagle Bu1}
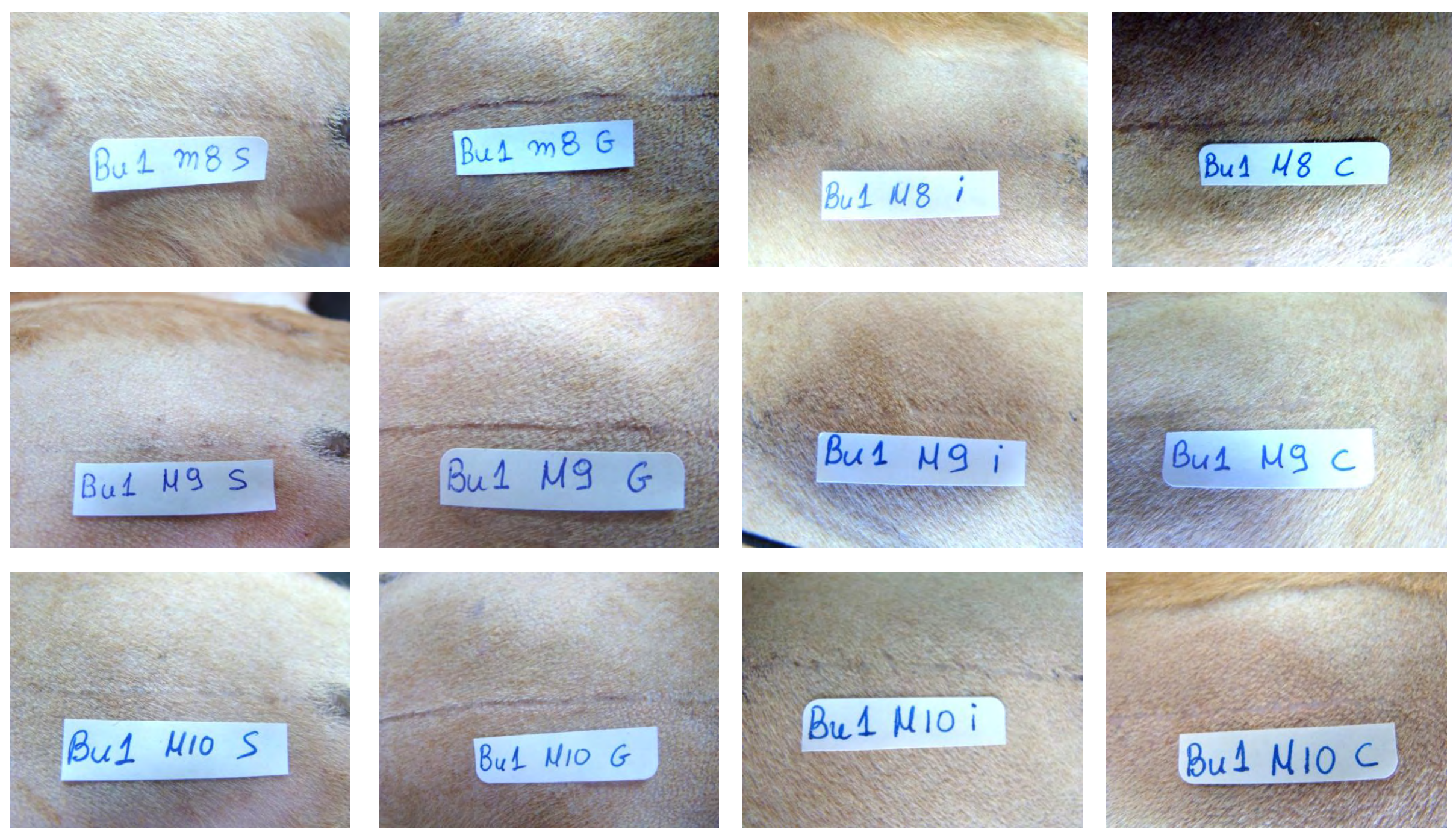


\section{Beagle Bu1}
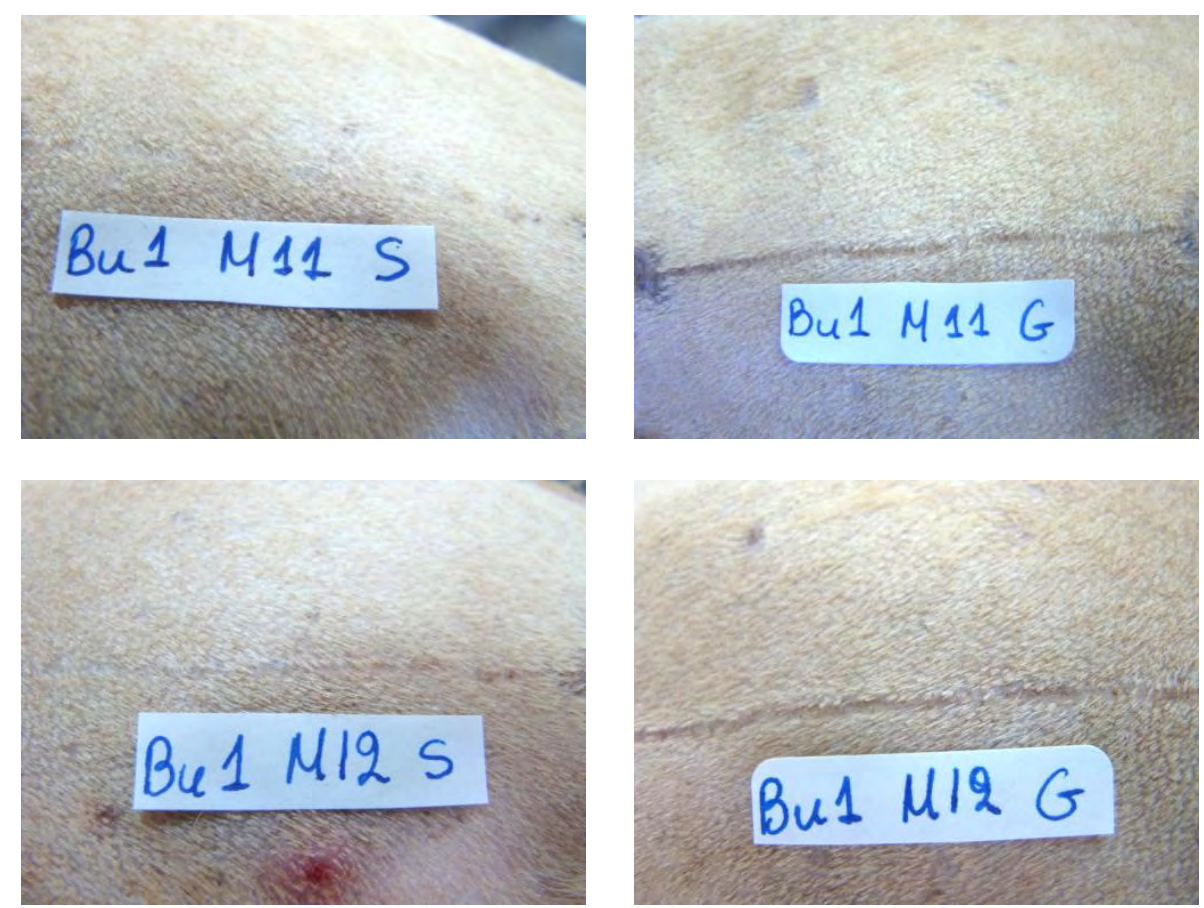

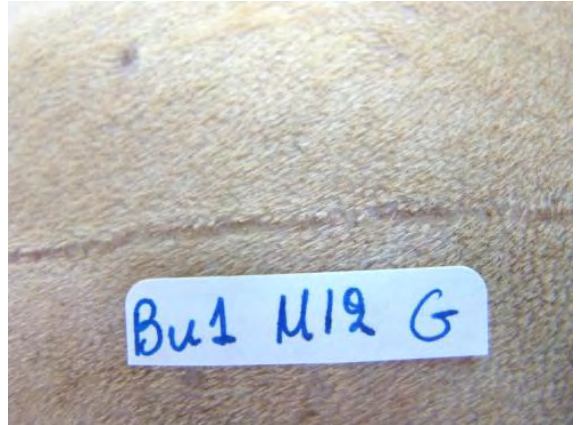

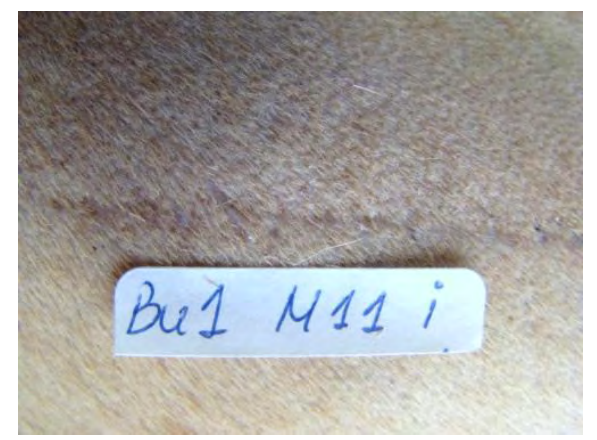
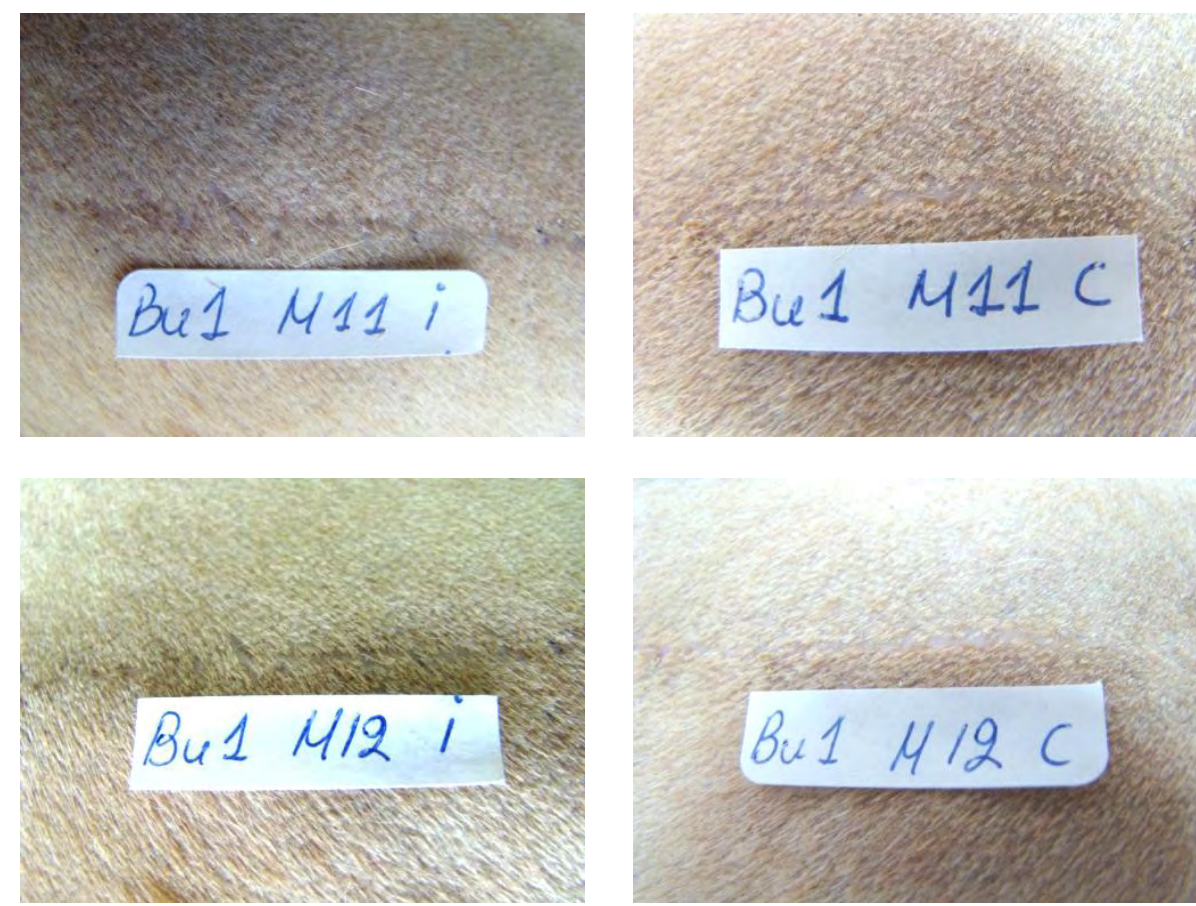

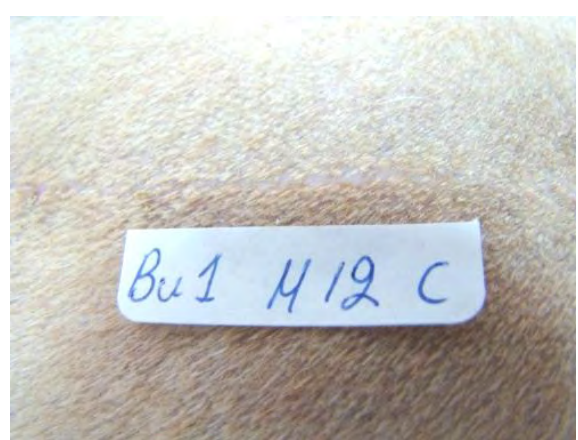




\section{Beagle Bu2}
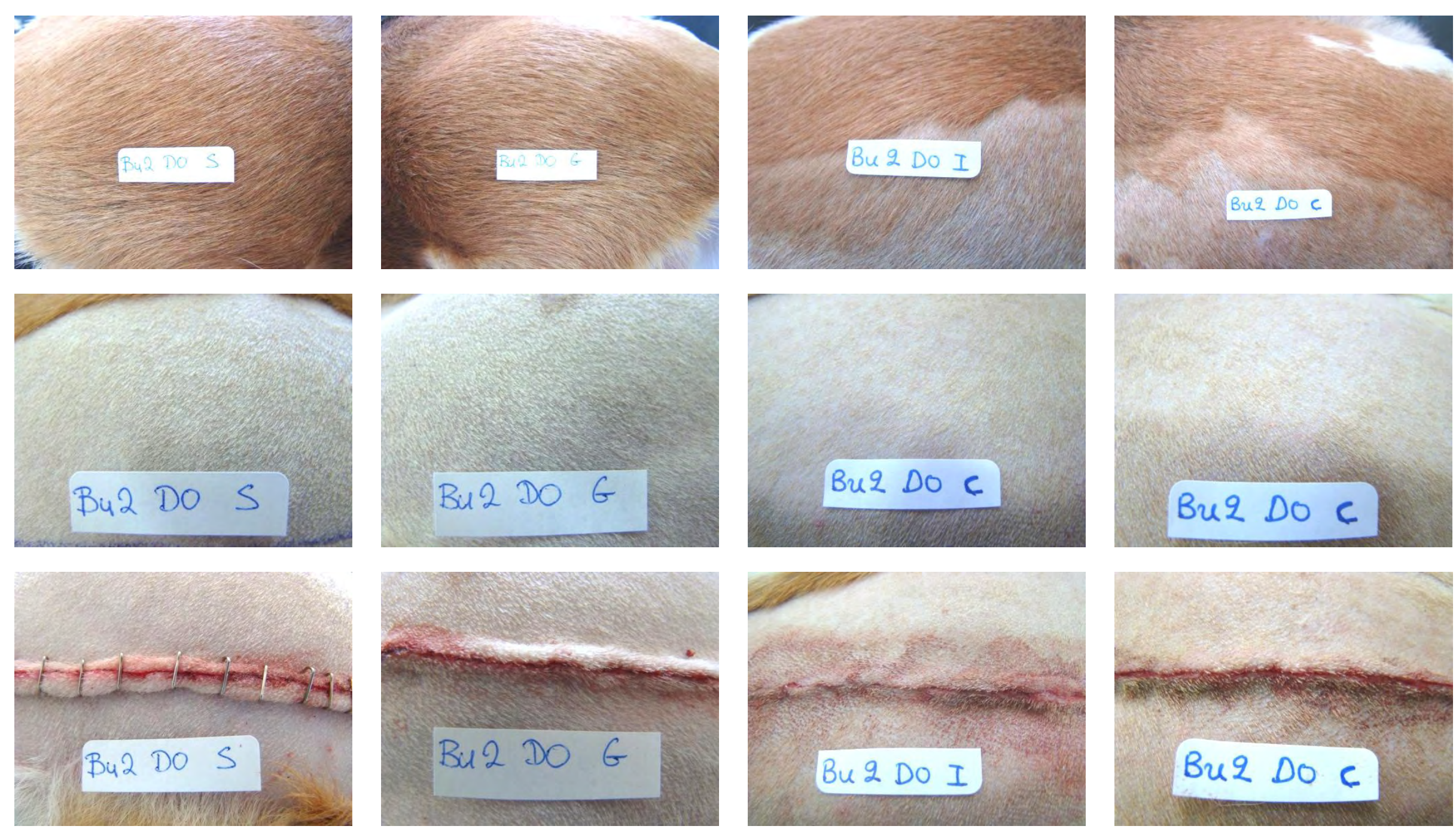


\section{Beagle Bu2}
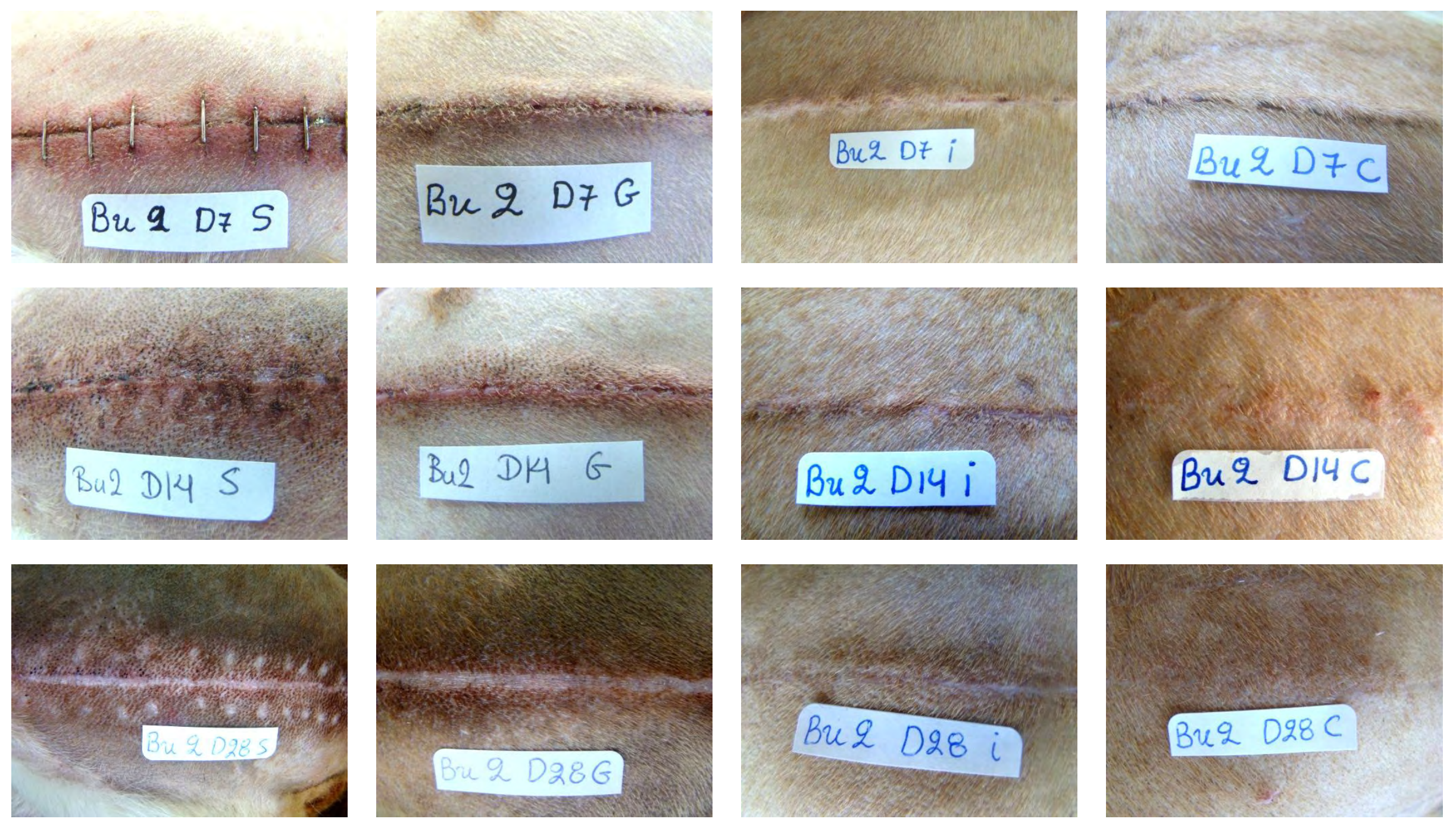


\section{Beagle Bu2}
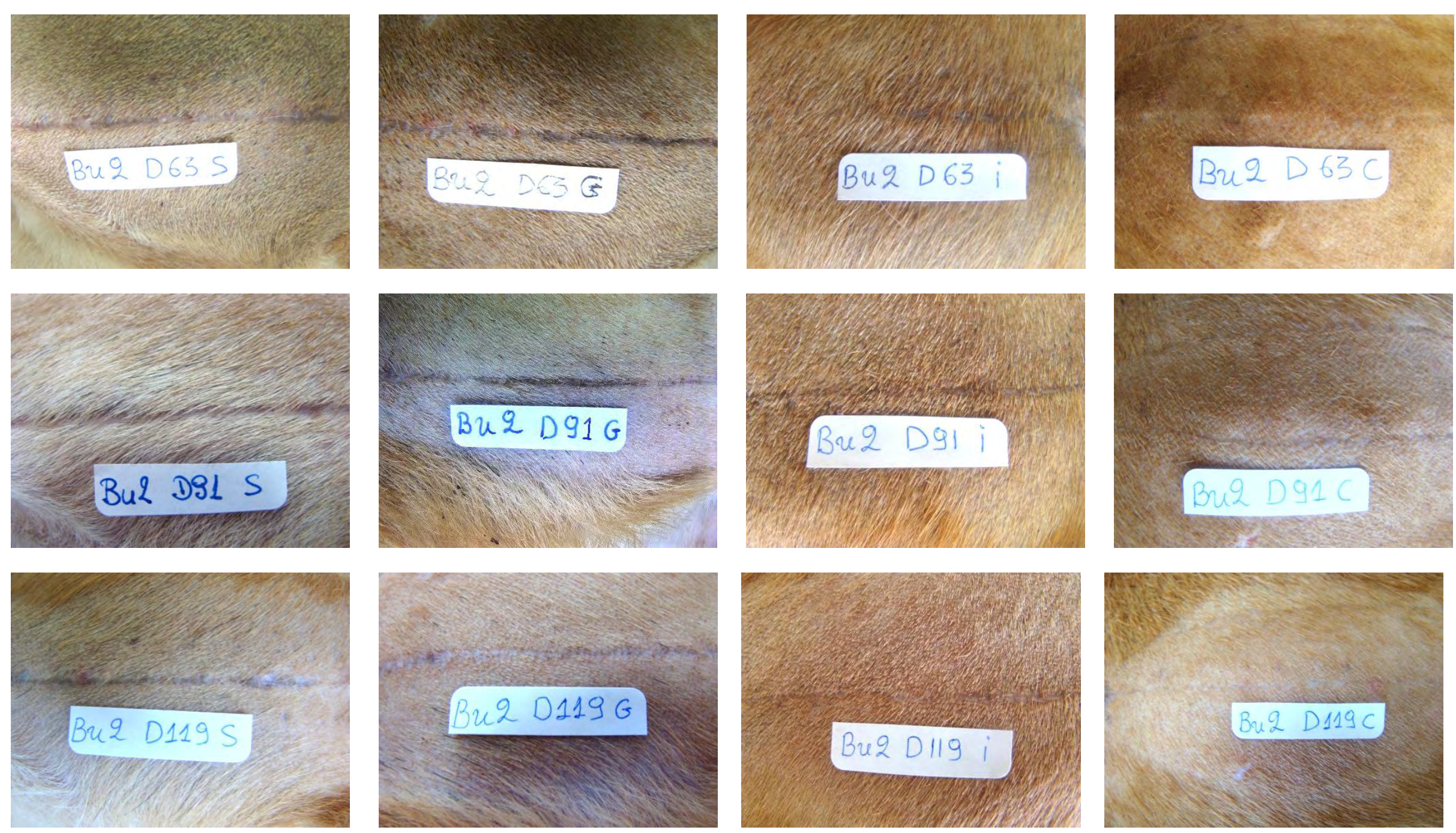


\section{Beagle Bu2}
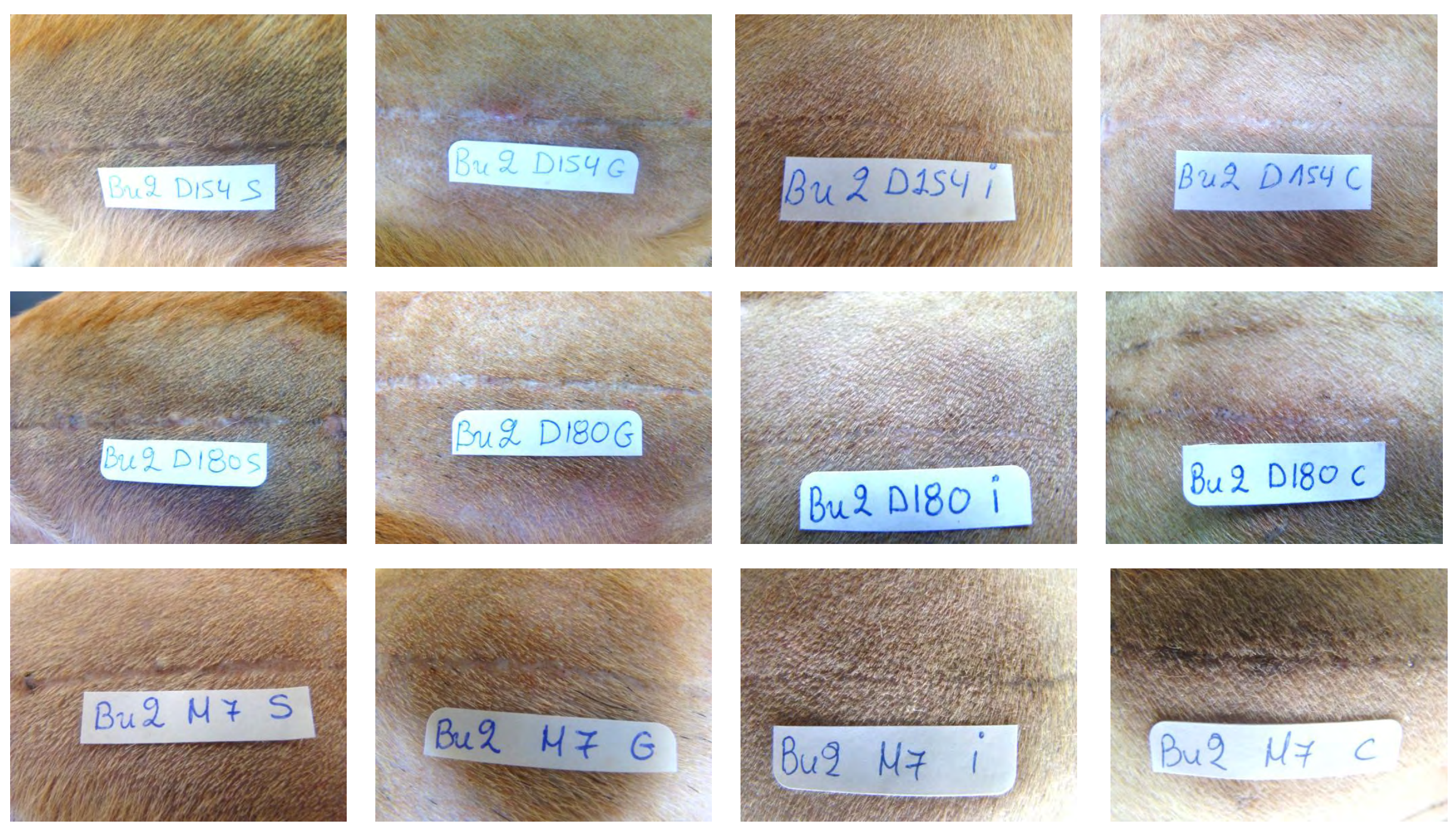


\section{Beagle Bu2}
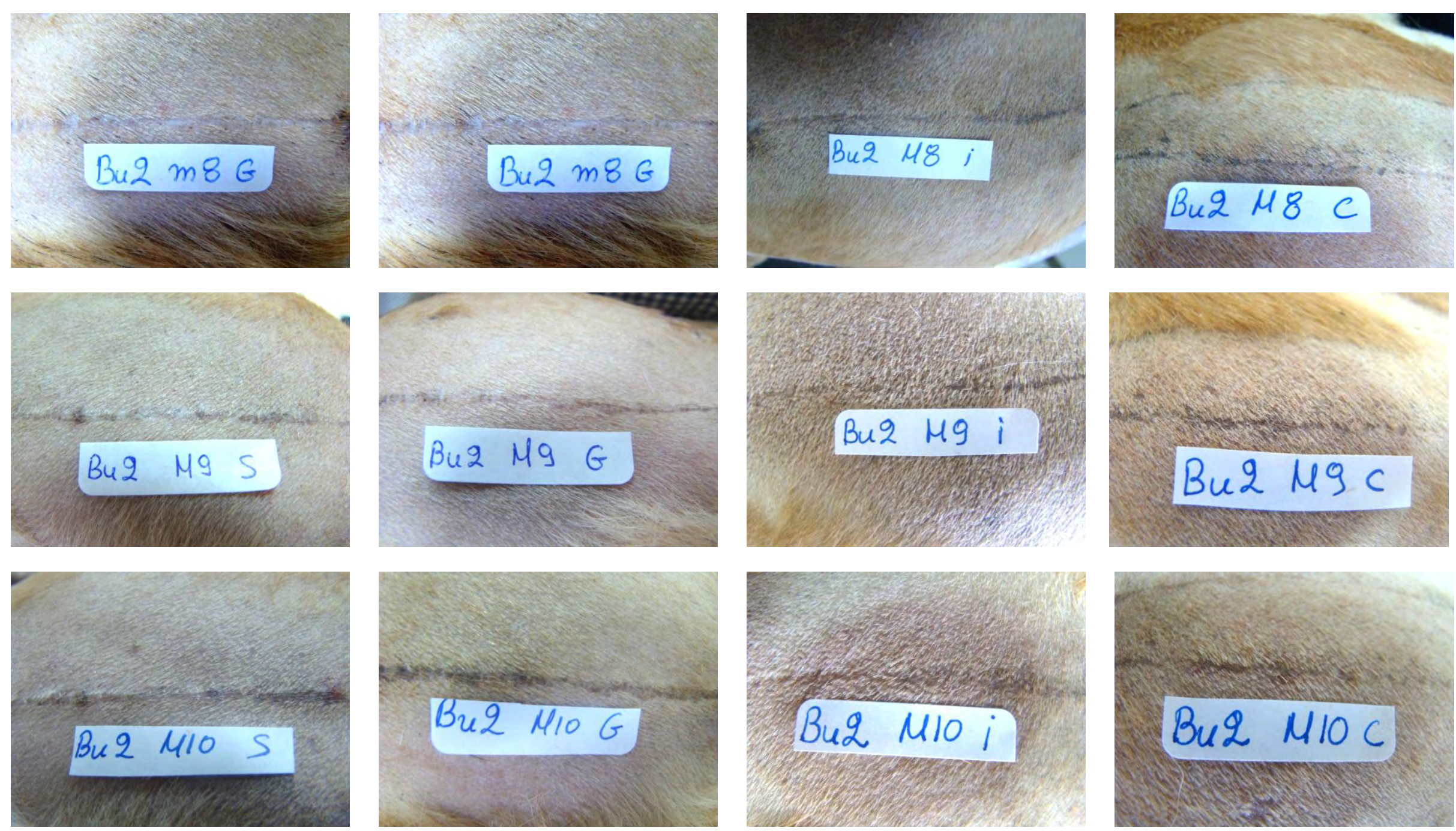


\section{Beagle Bu2}
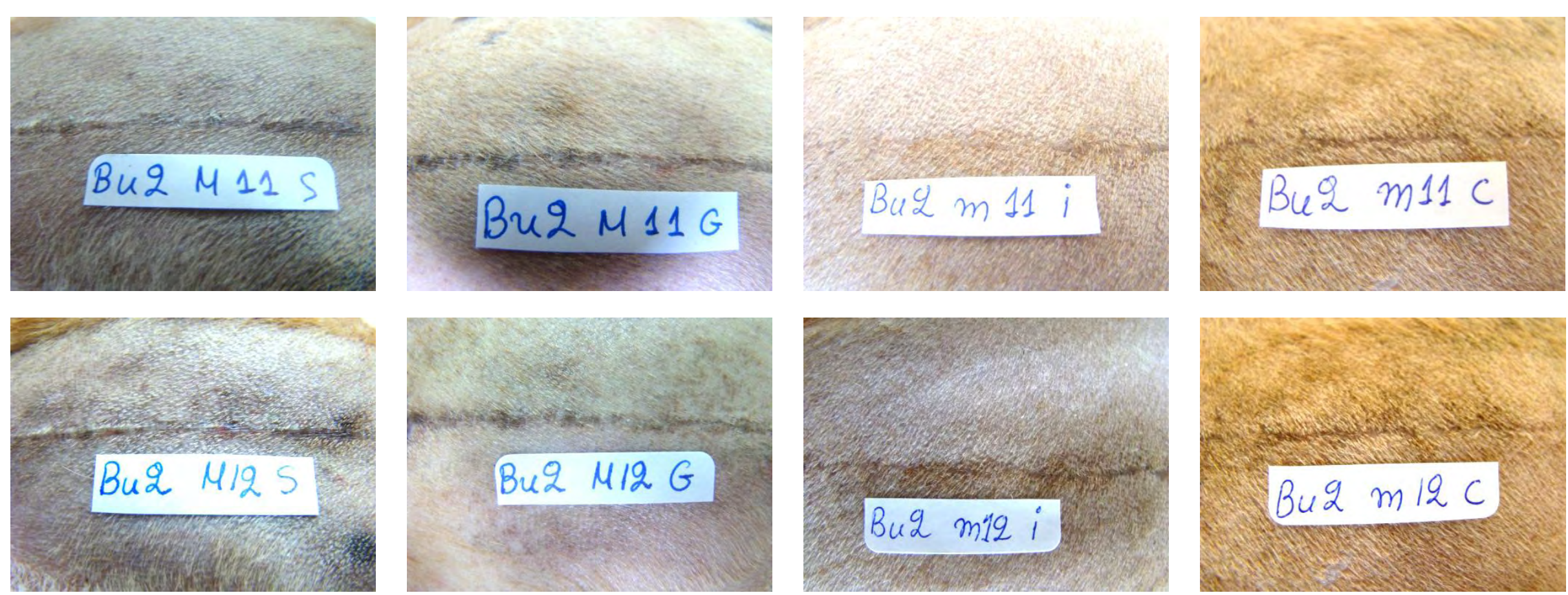
Beagle Bu3
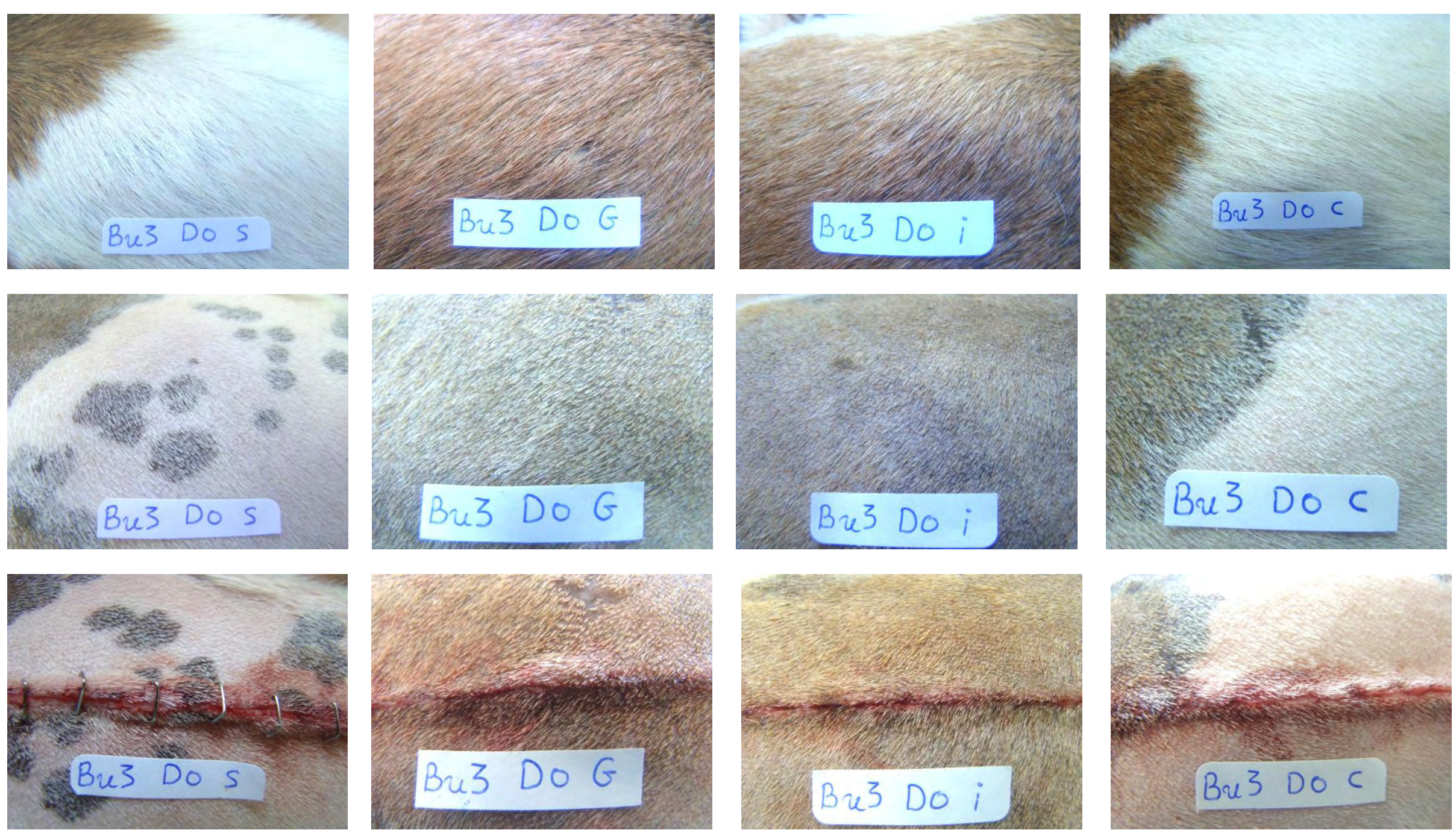


\section{Beagle Bu3}
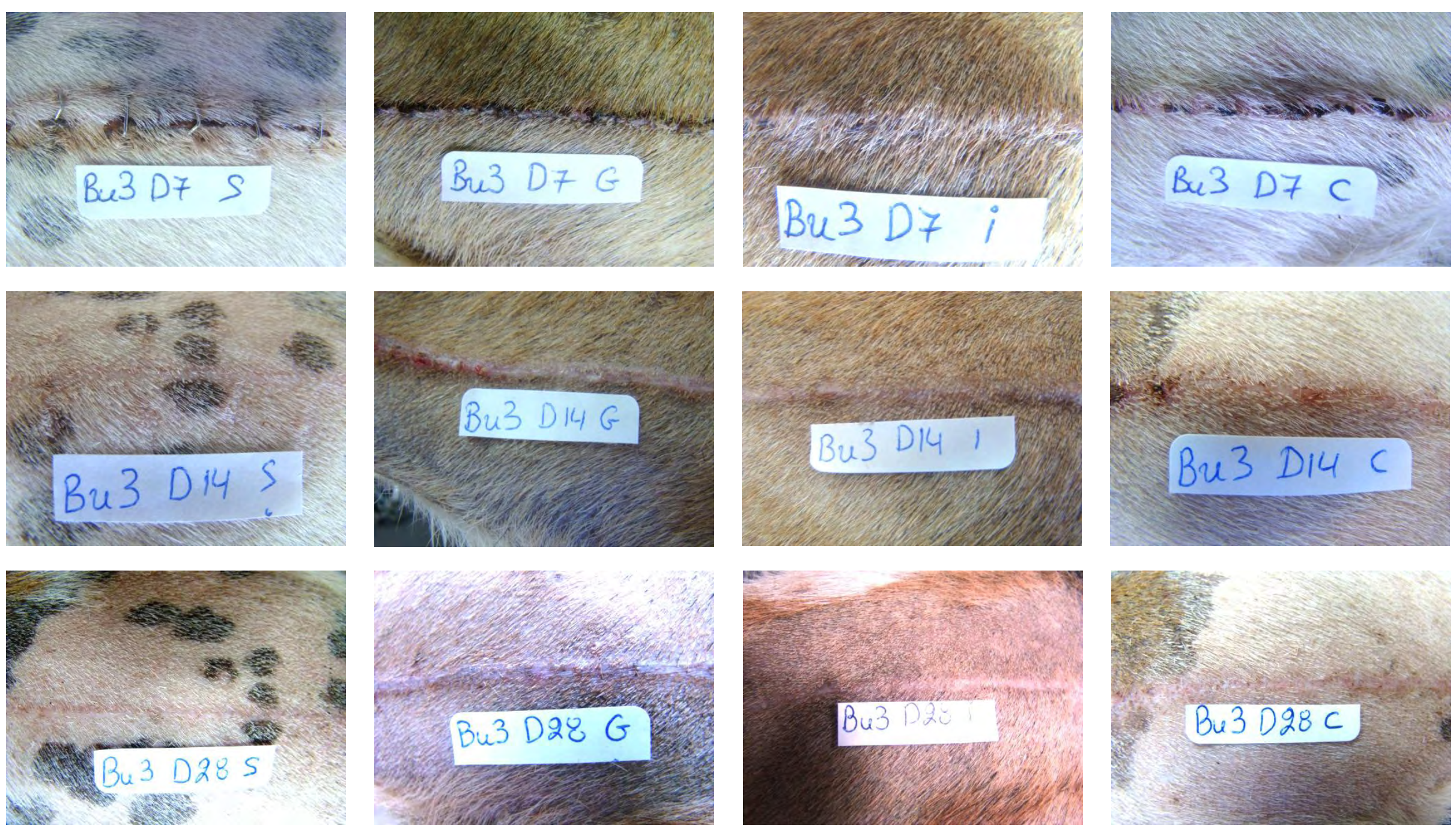
Beagle Bu3
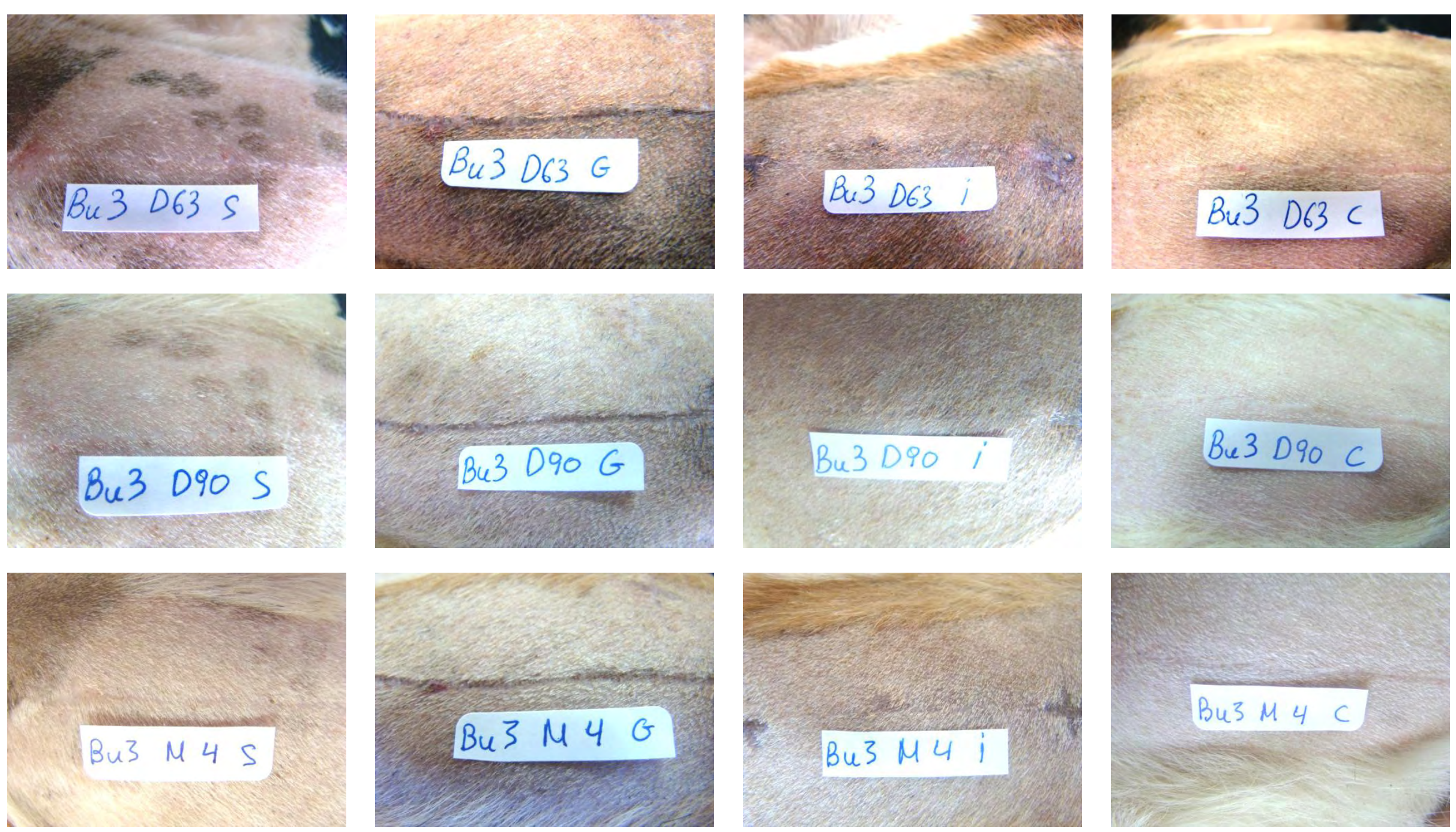


\section{Beagle Bu3}
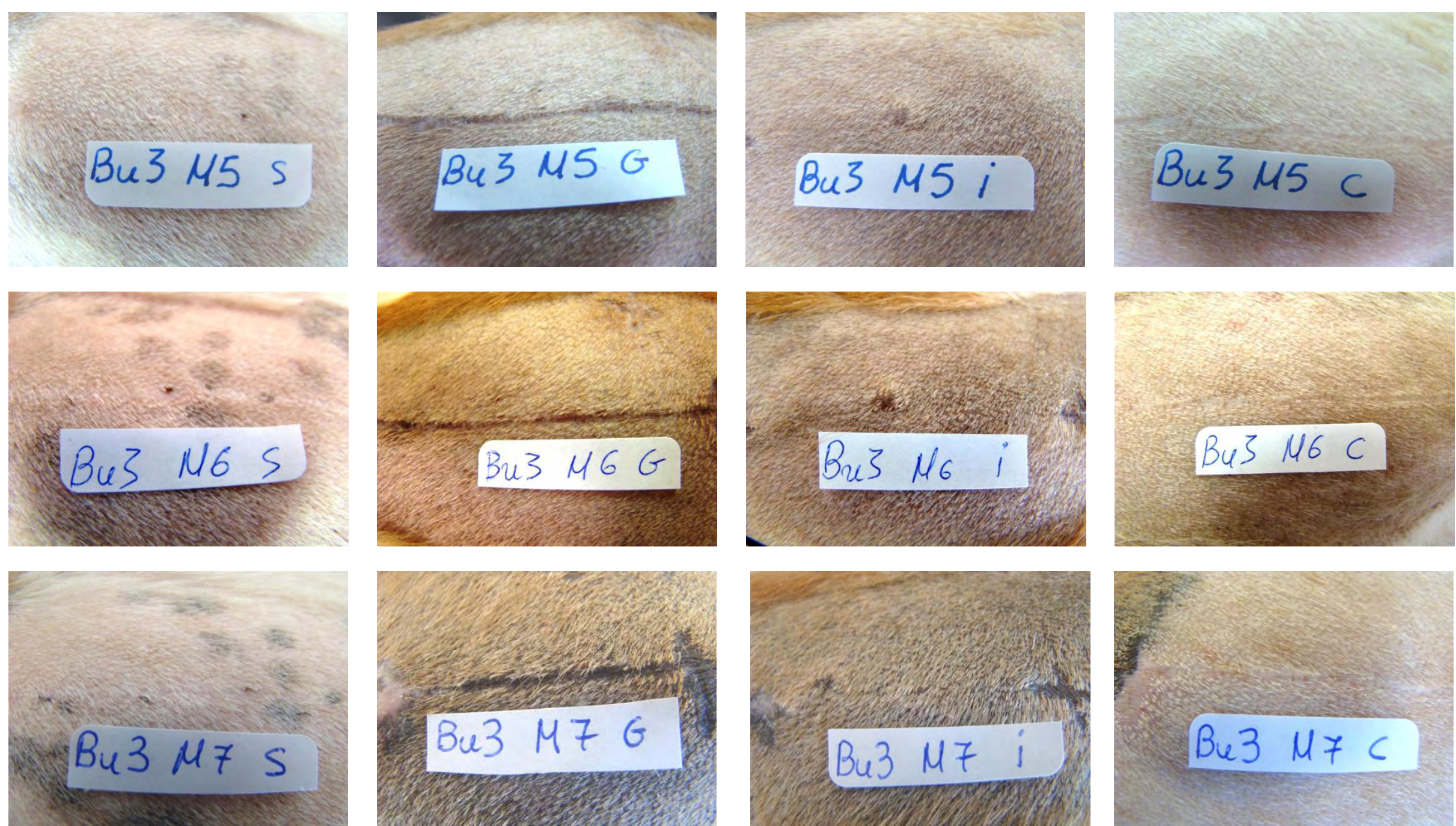
Beagle Bu3
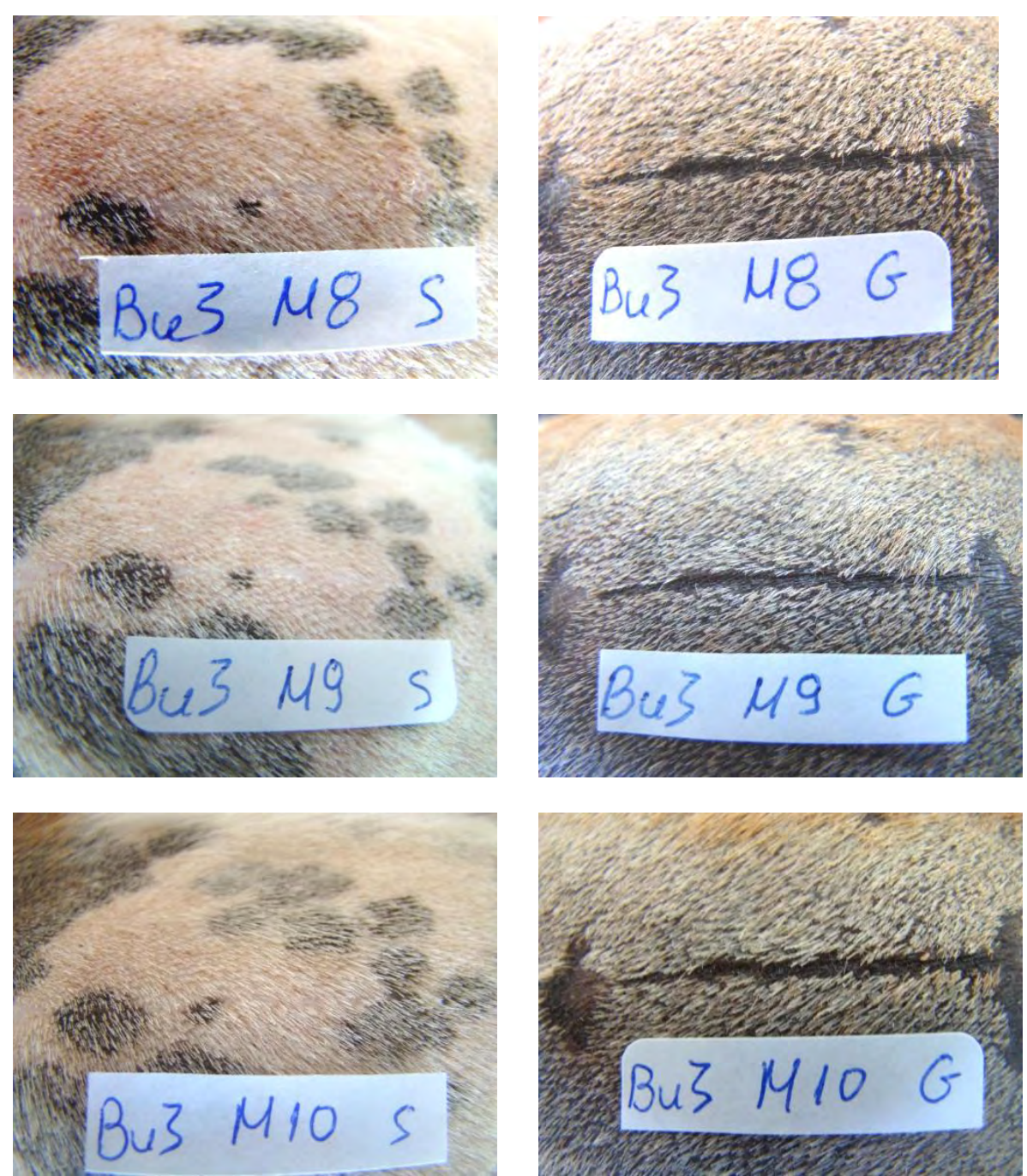
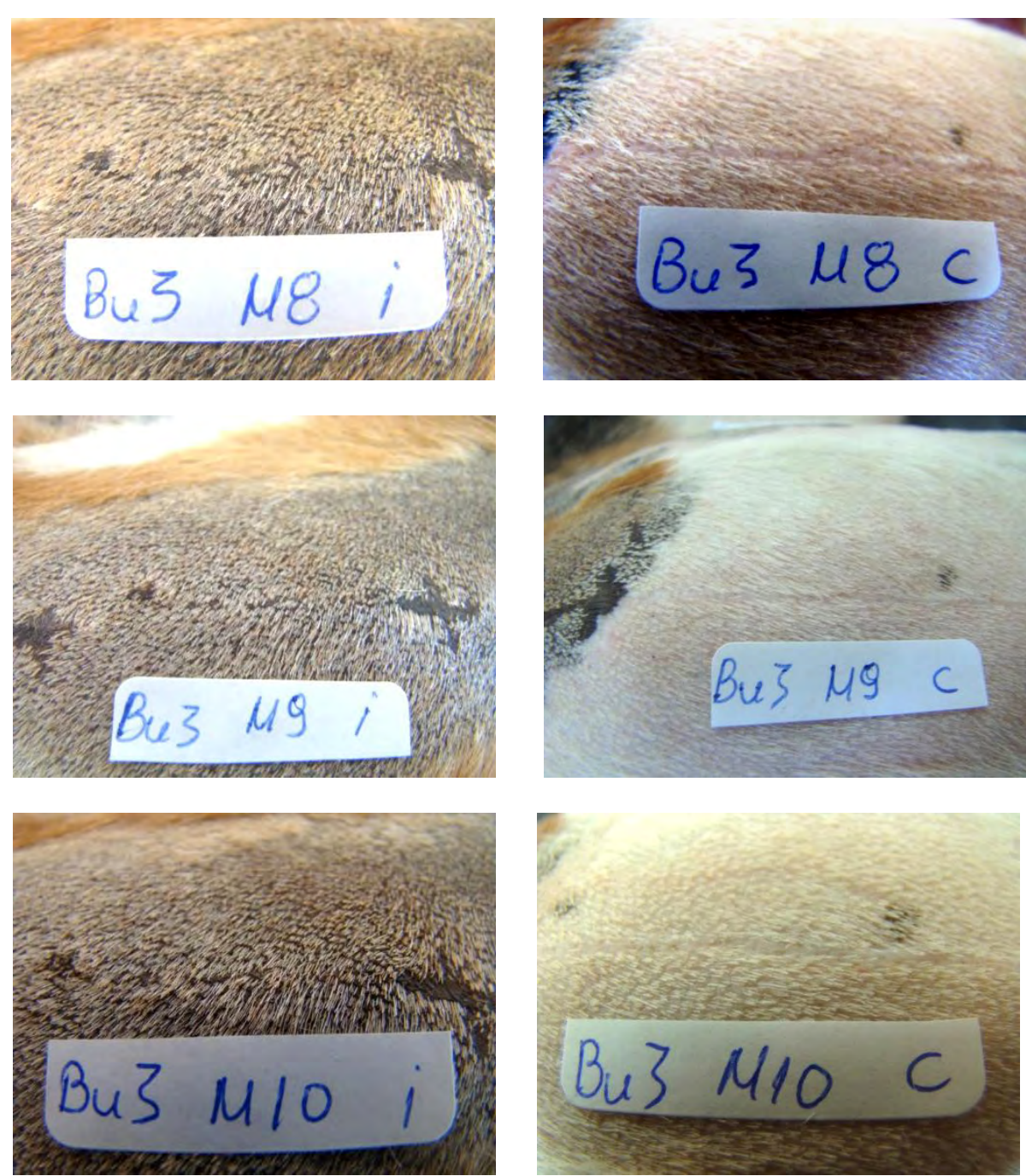


\section{Beagle Bu3}
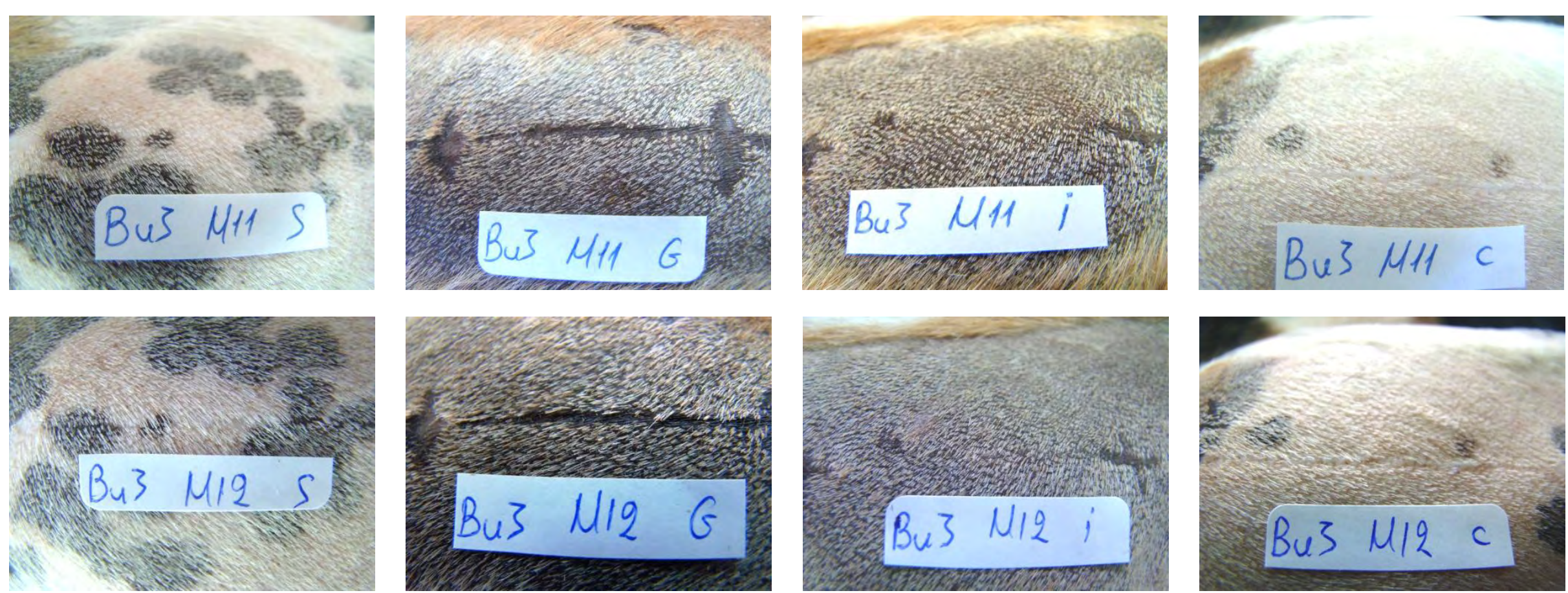
Beagle Bu4
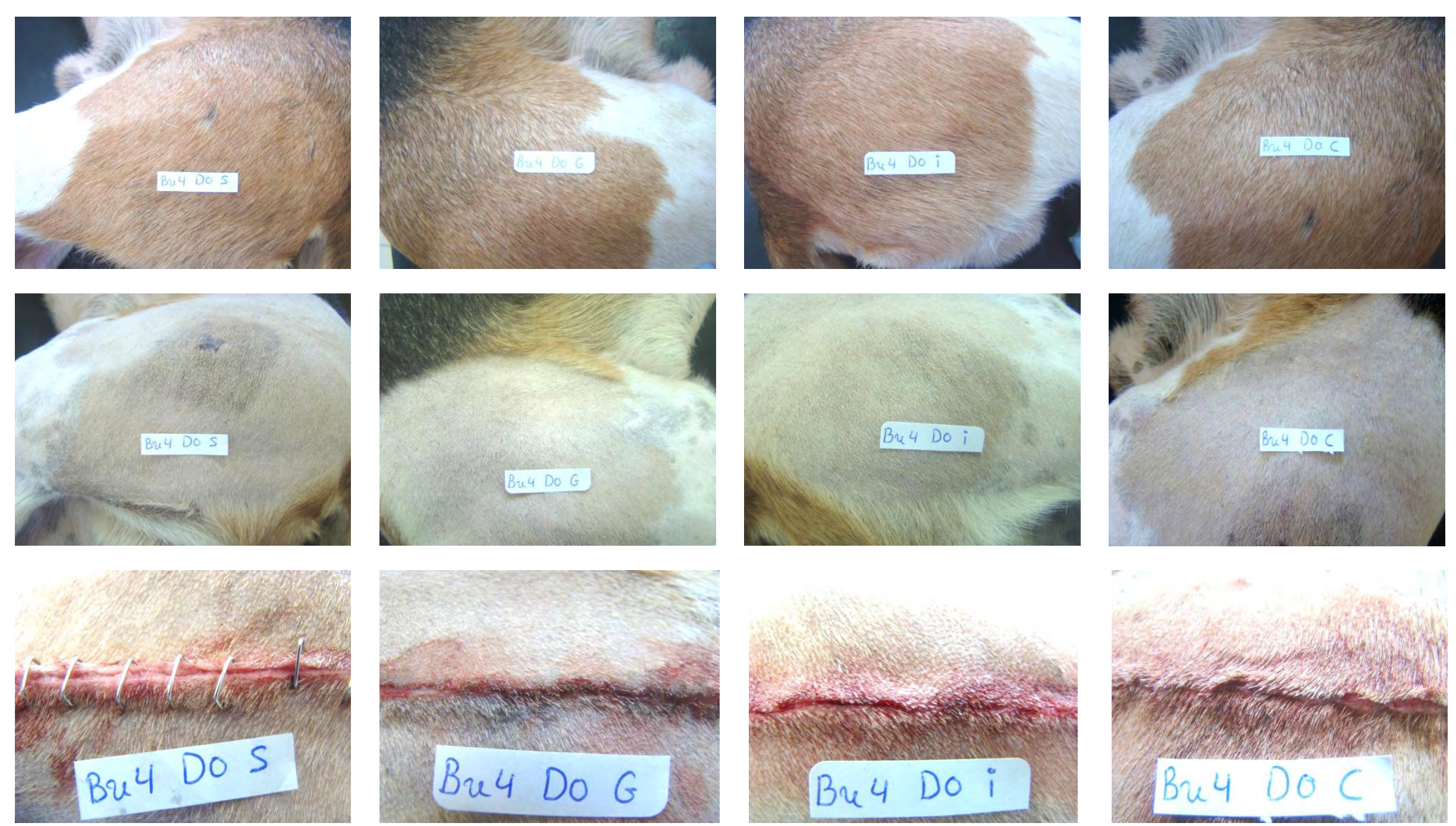


\section{Beagle Bu4}
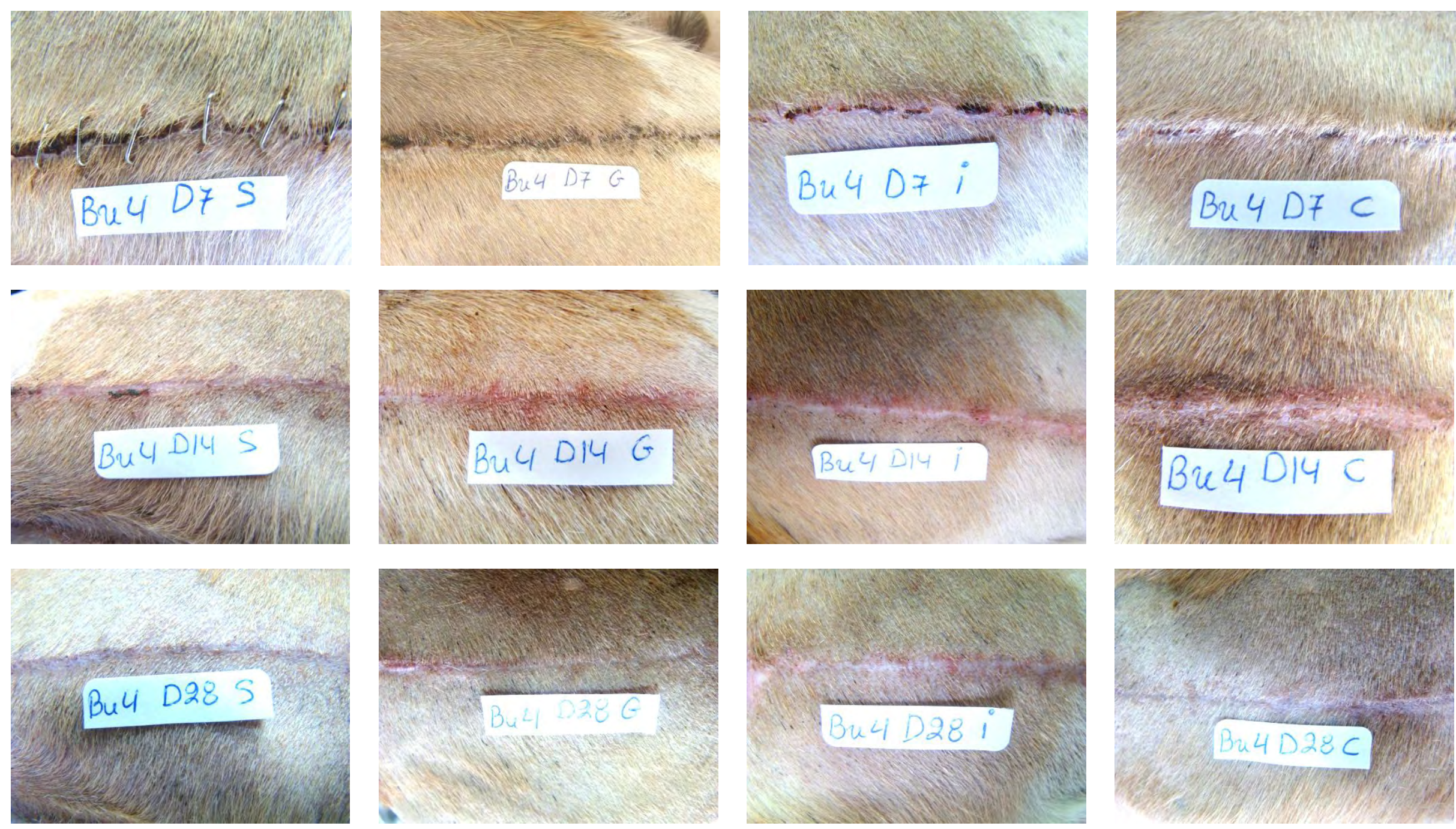


\section{Beagle Bu4}
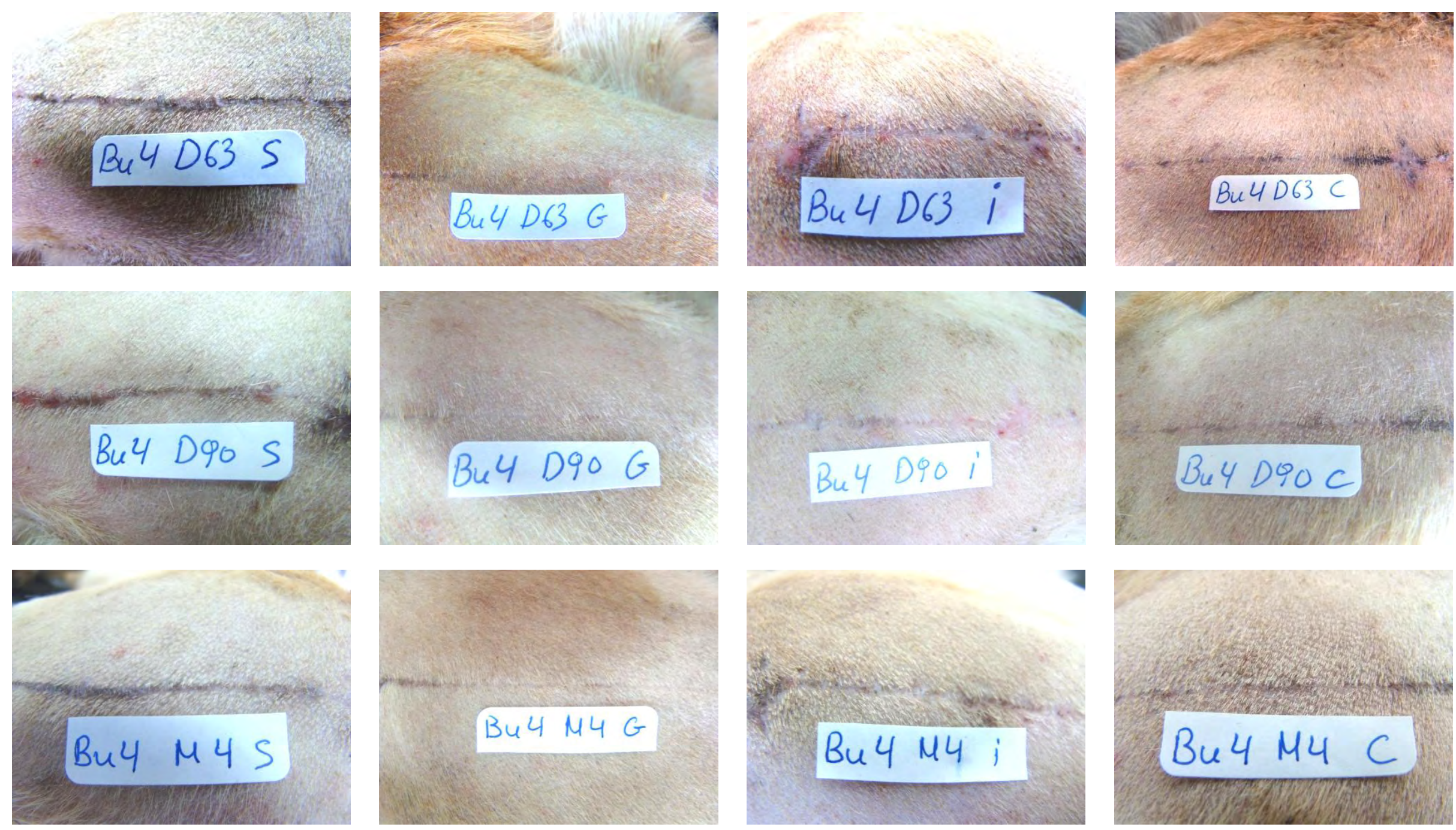


\section{Beagle Bu4}
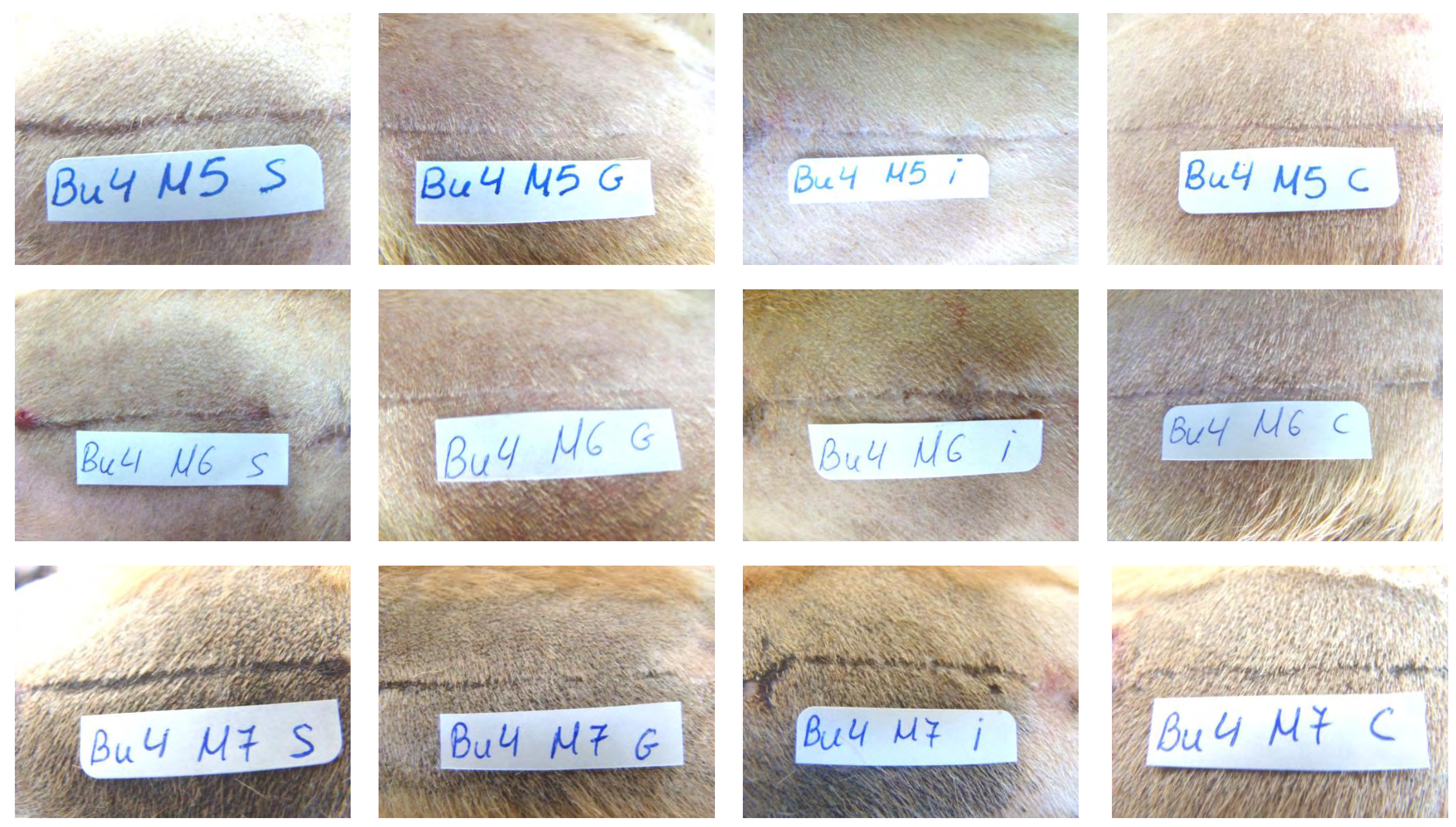


\section{Beagle Bu4}
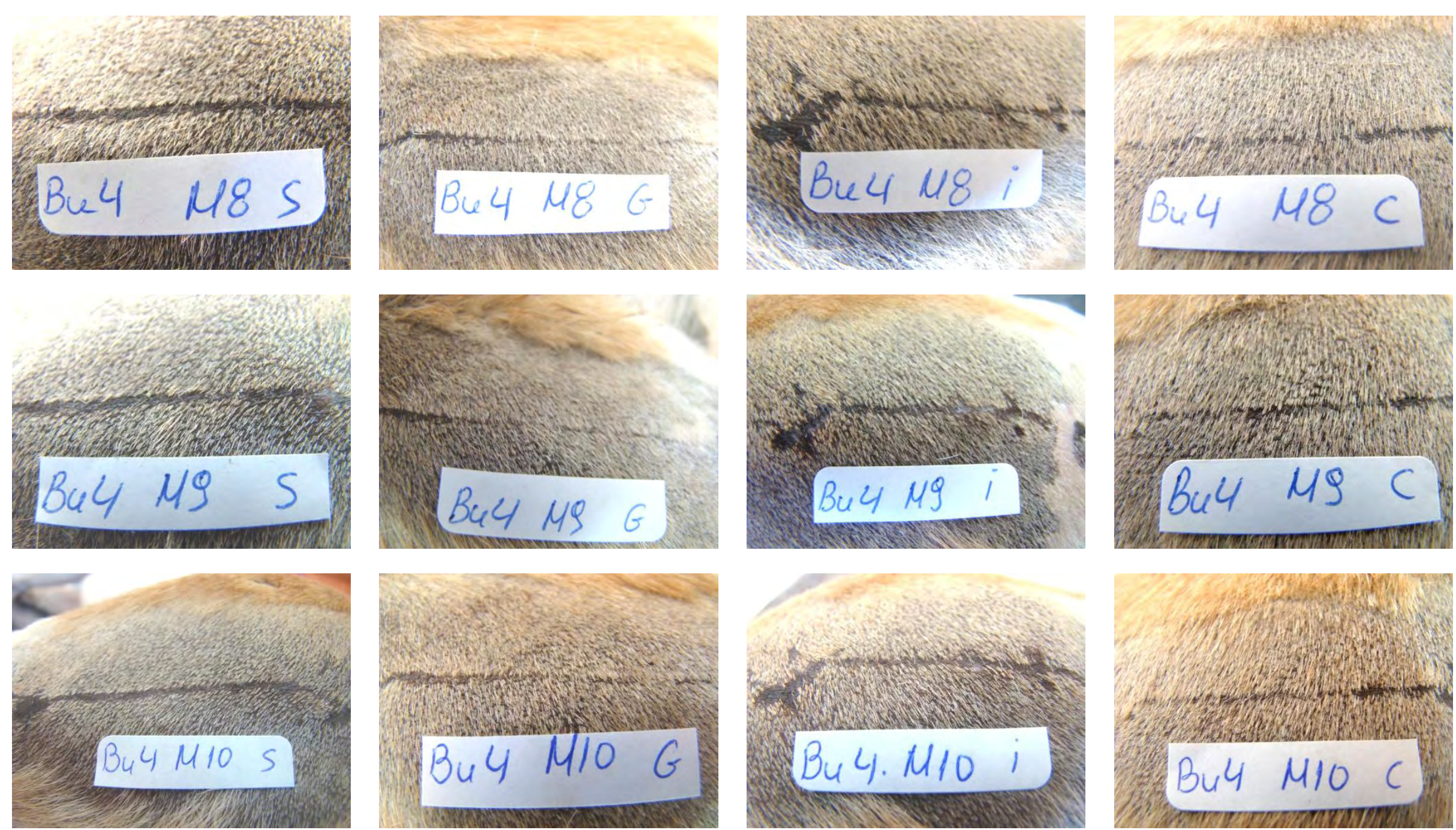


\section{Beagle Bu4}
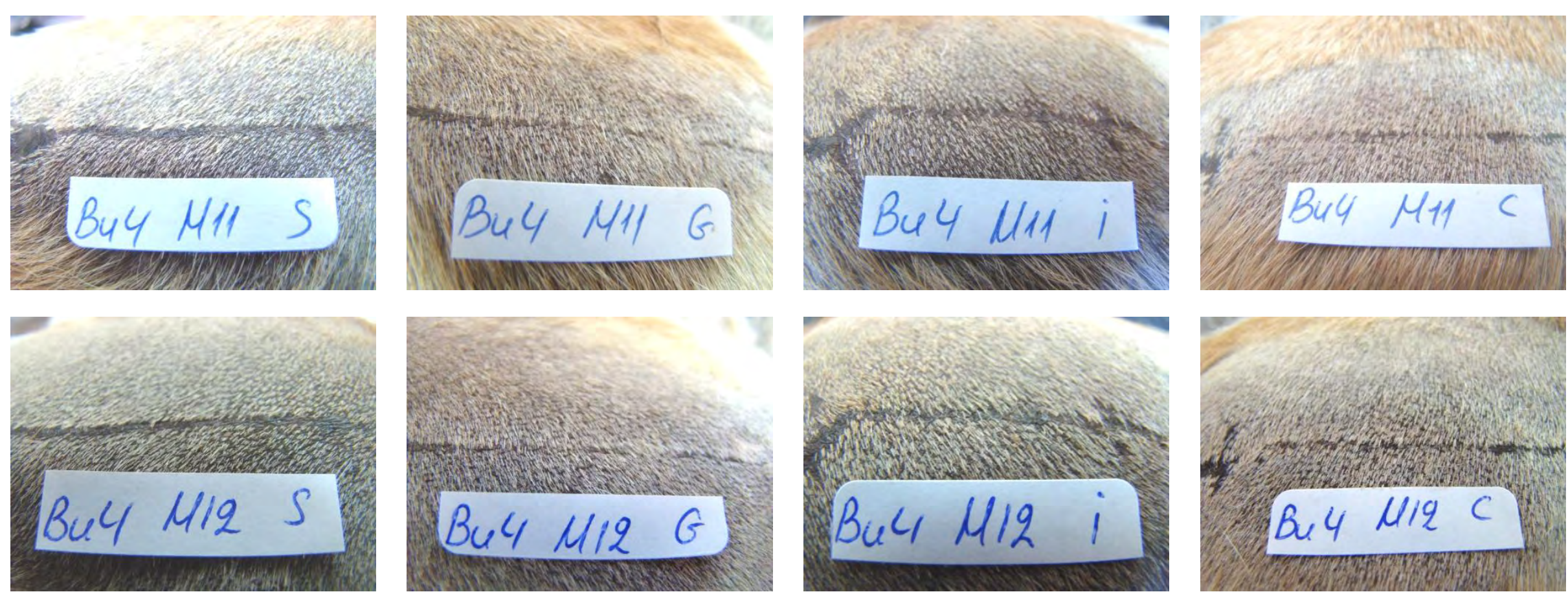
Beagle Bu5
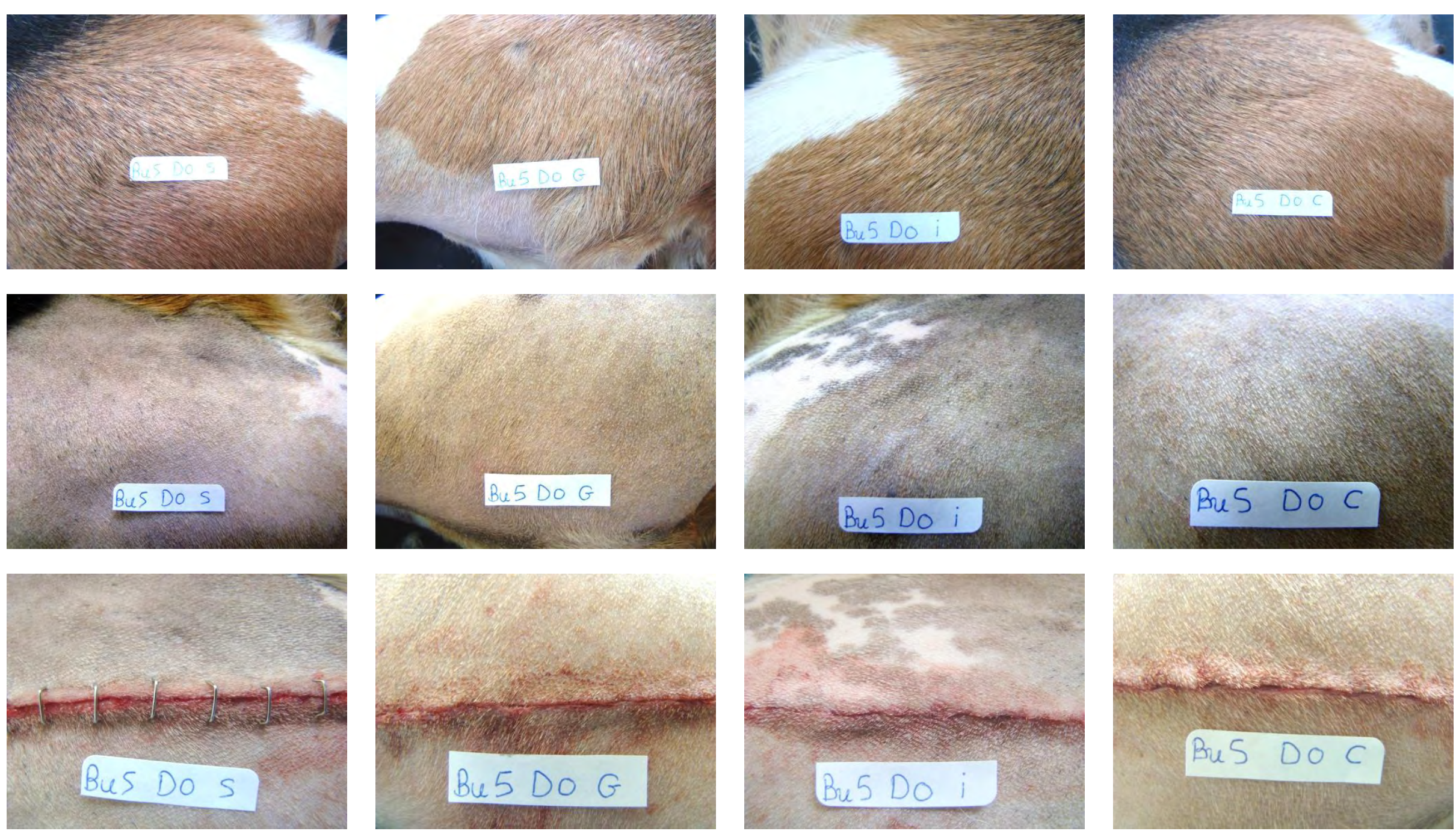


\section{Beagle Bu5}
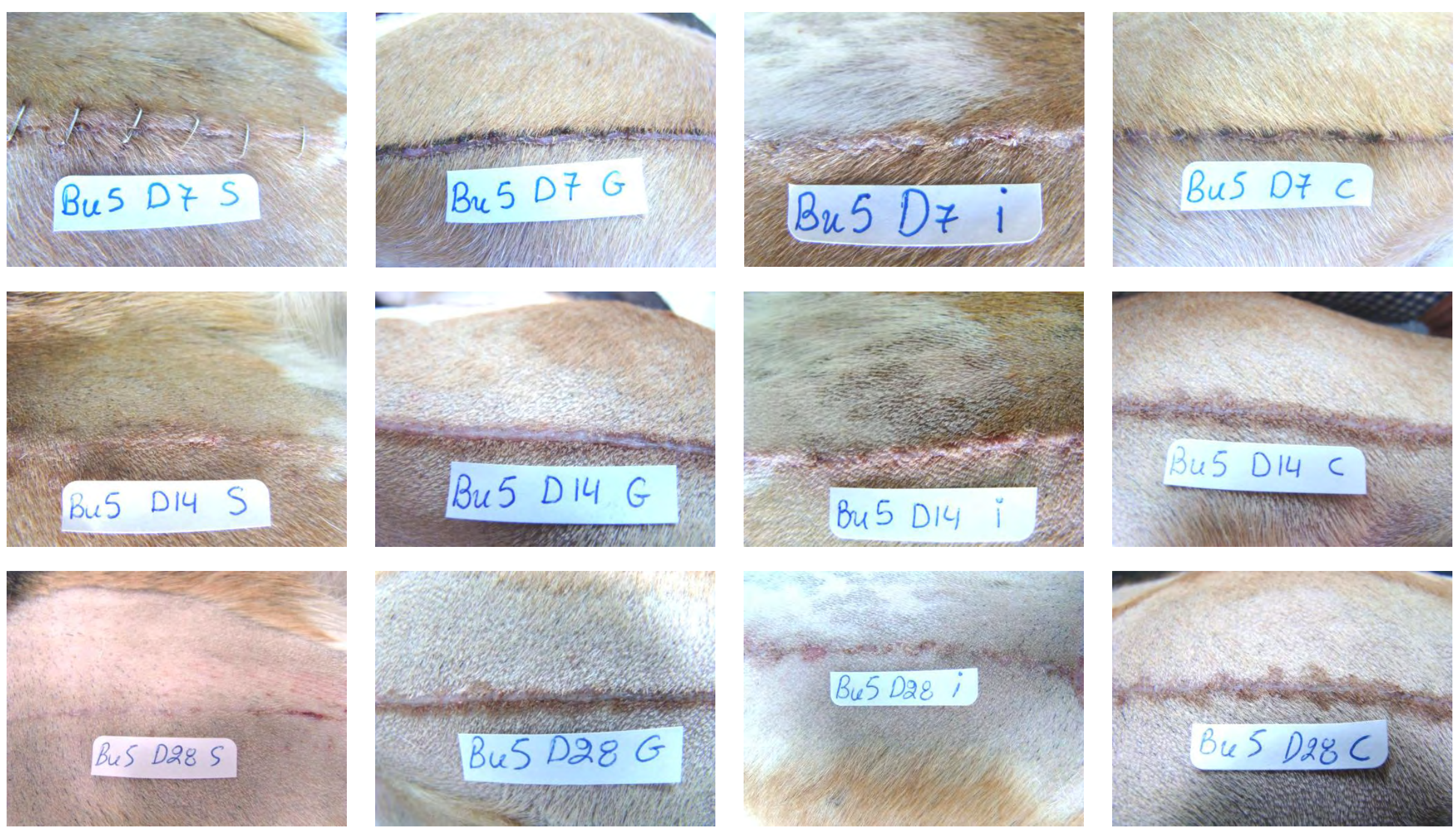
Beagle Bu5
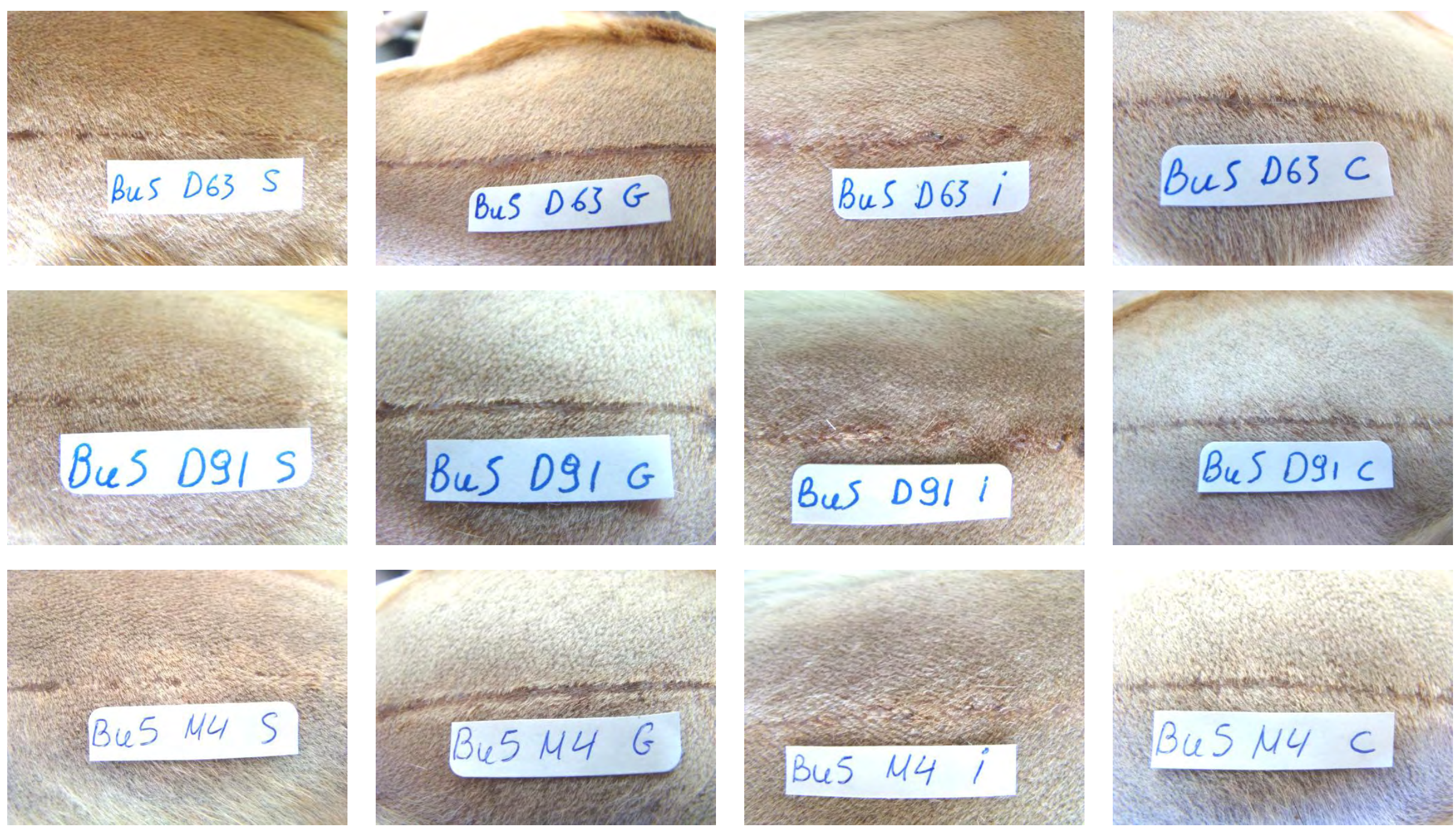


\section{Beagle Bu5}
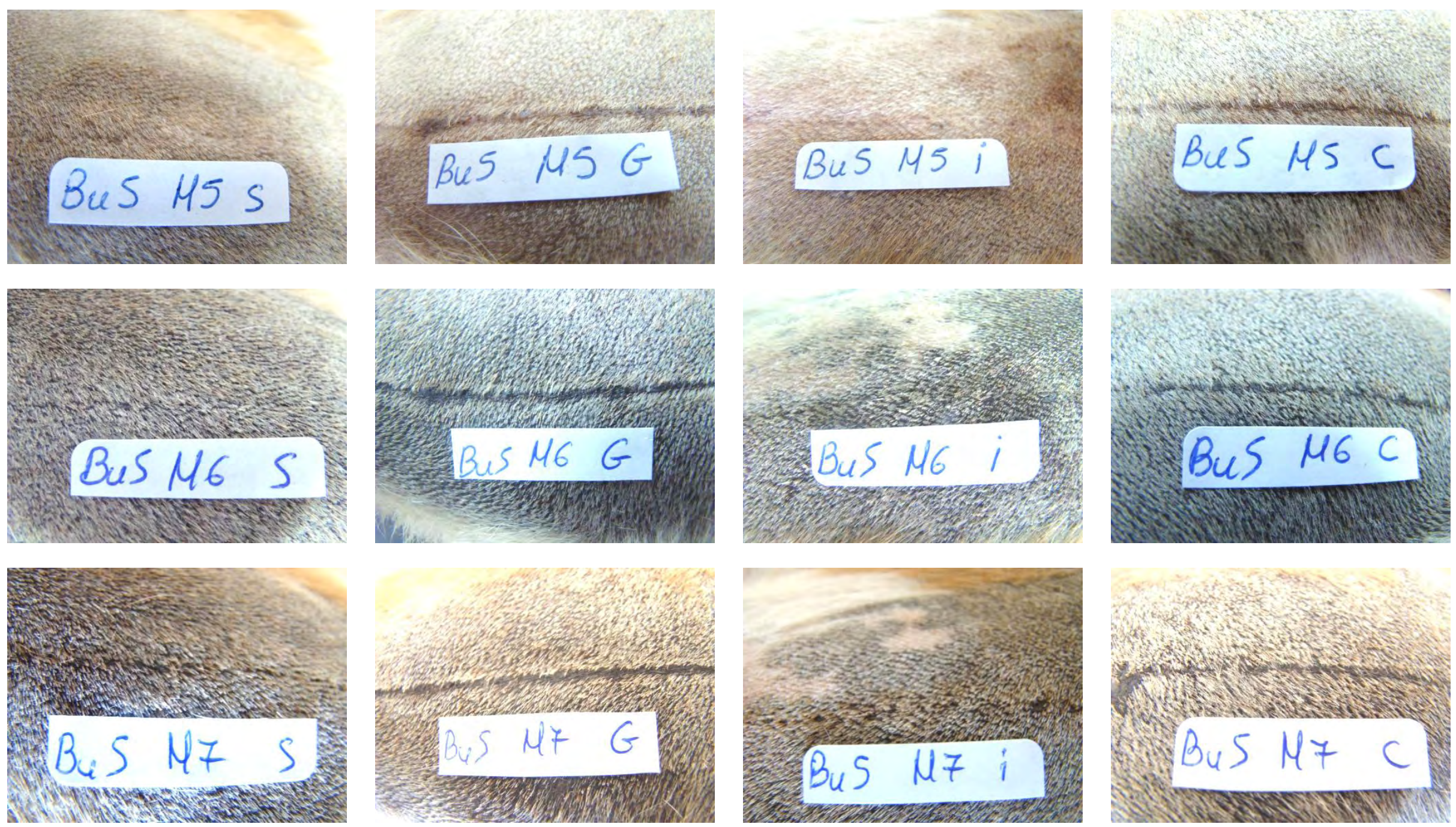


\section{Beagle Bu5}
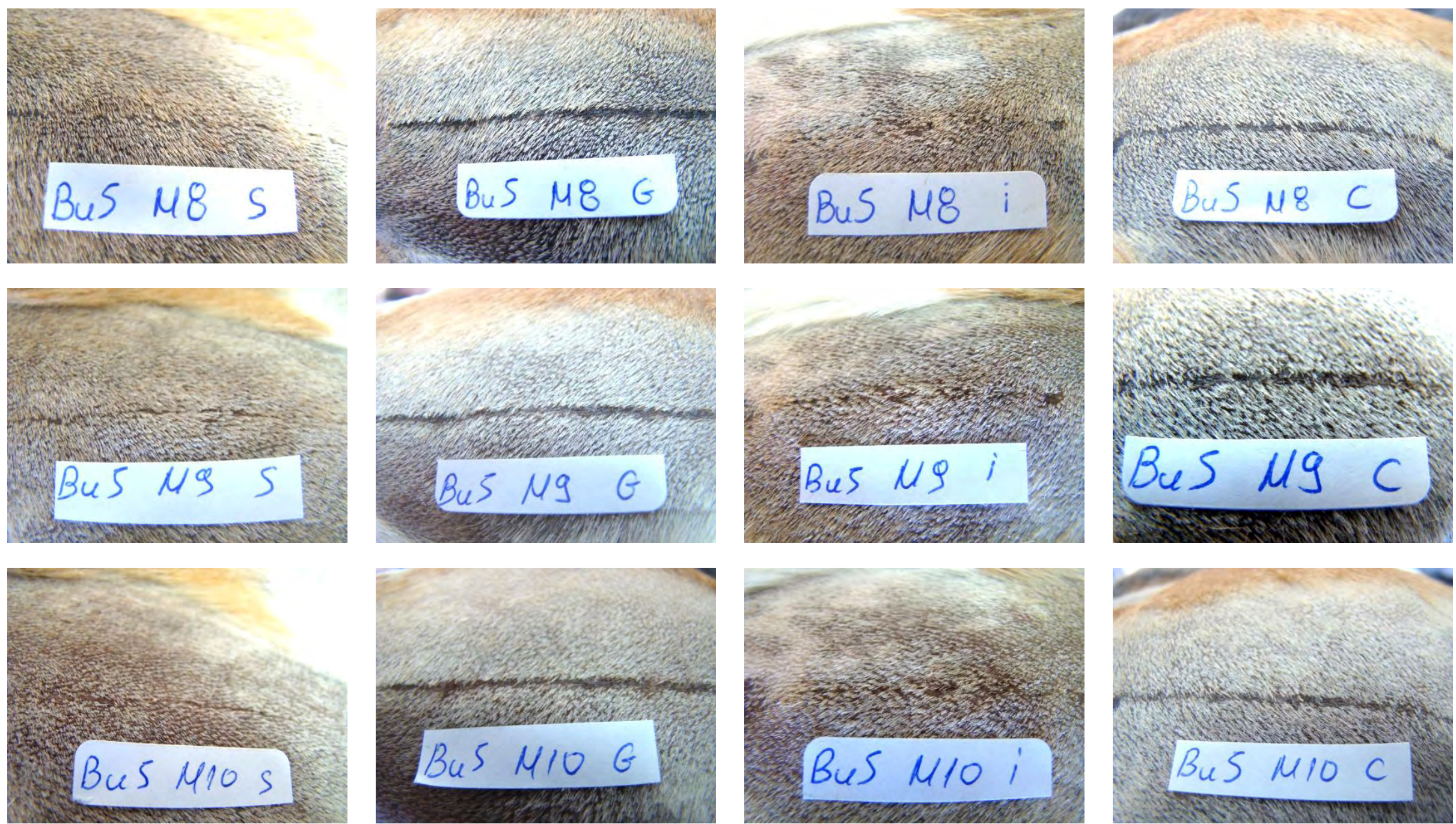


\section{Beagle Bu5}
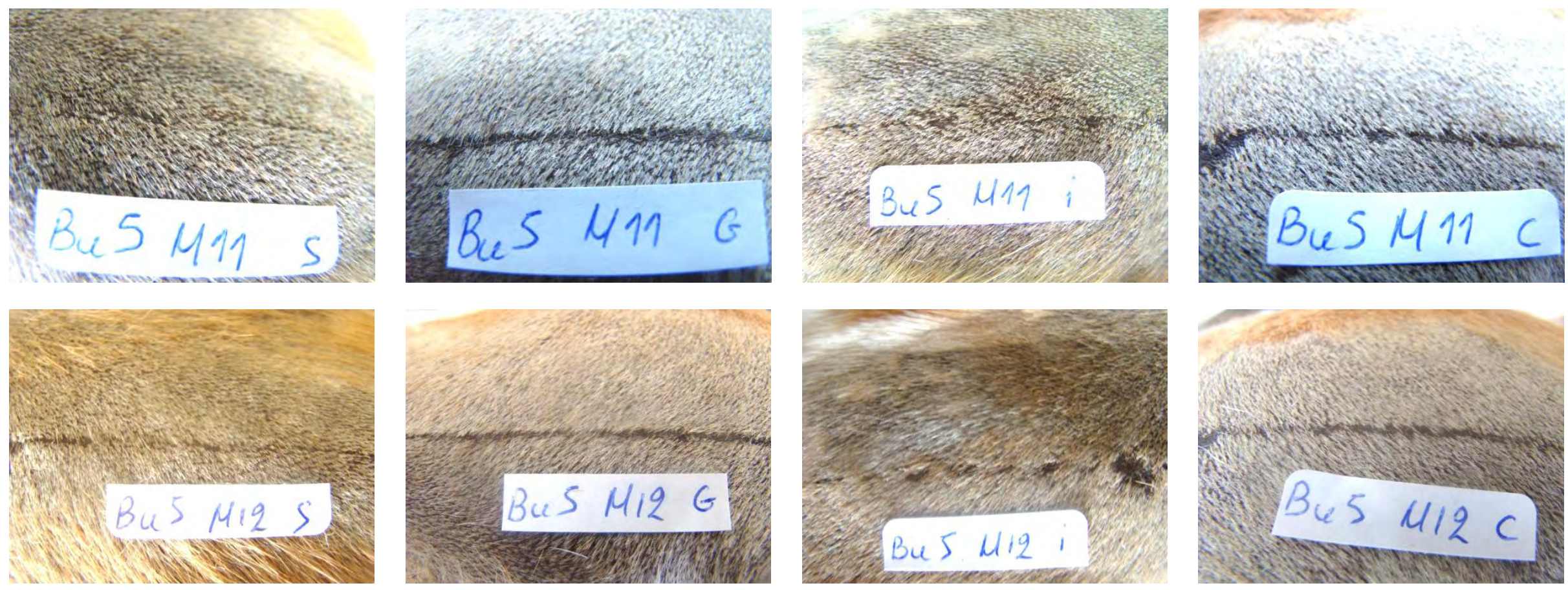


\section{Beagle Bu6}
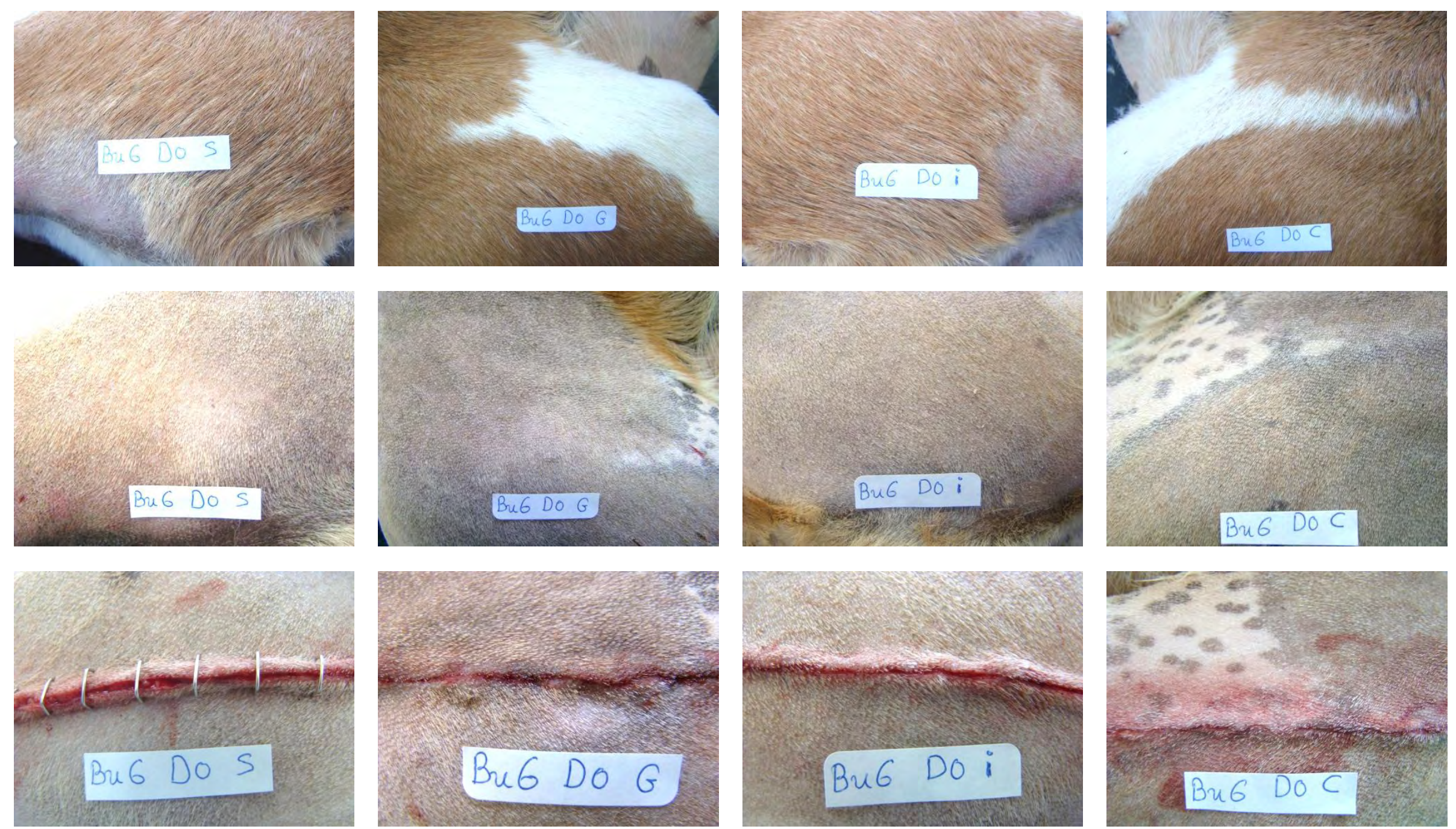


\section{Beagle Bu6}
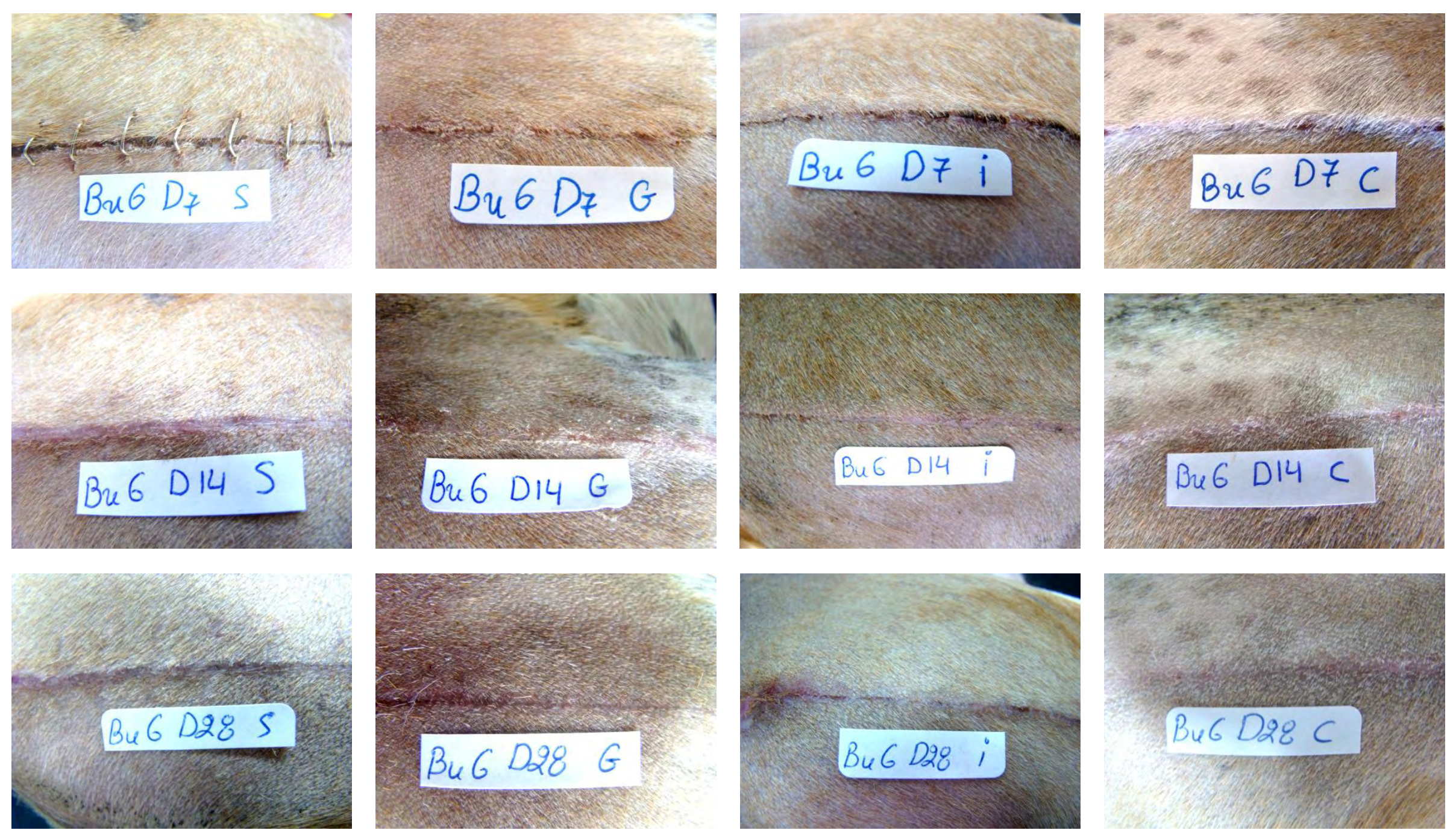


\section{Beagle Bu6}
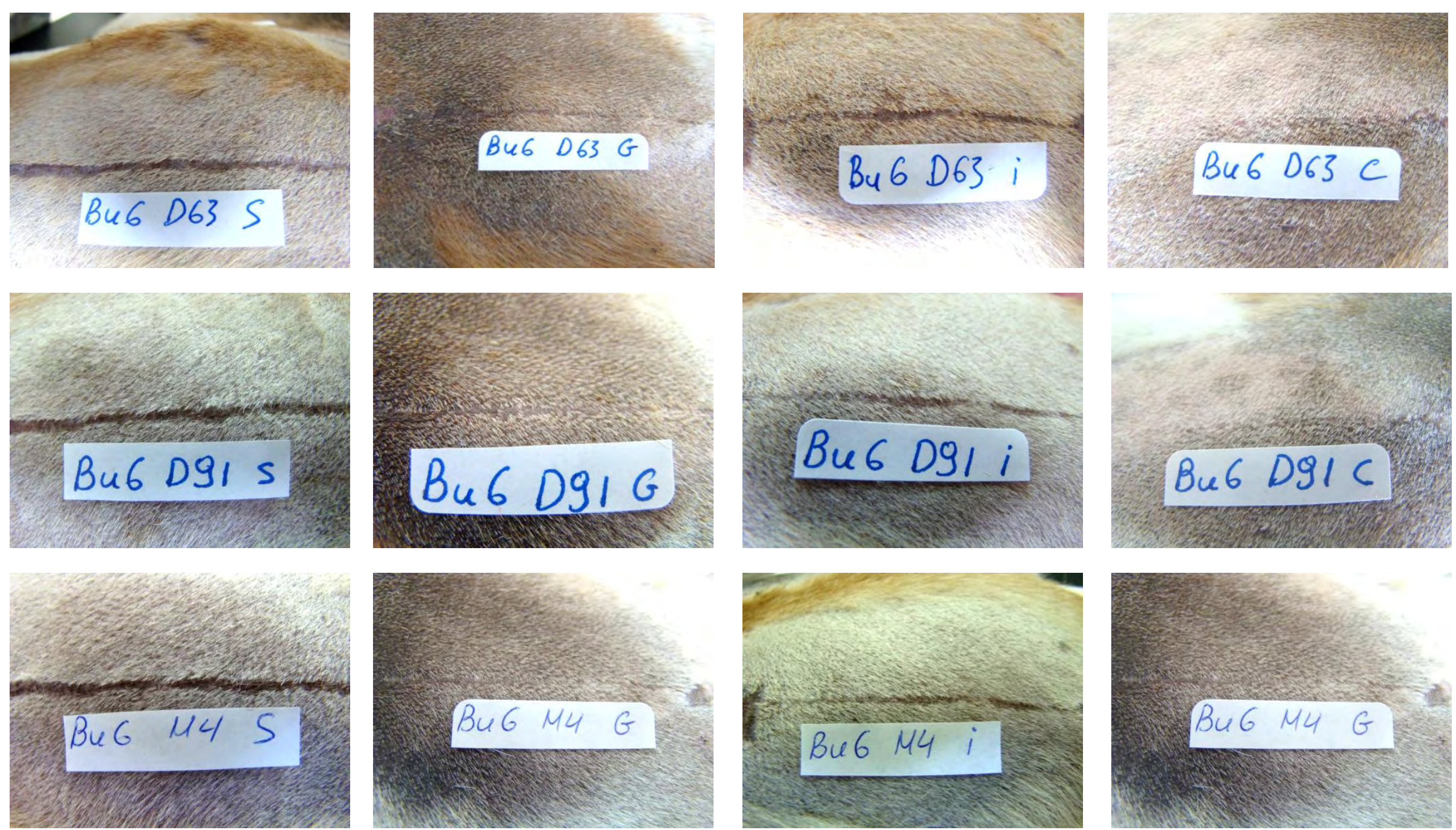
Beagle Bu6
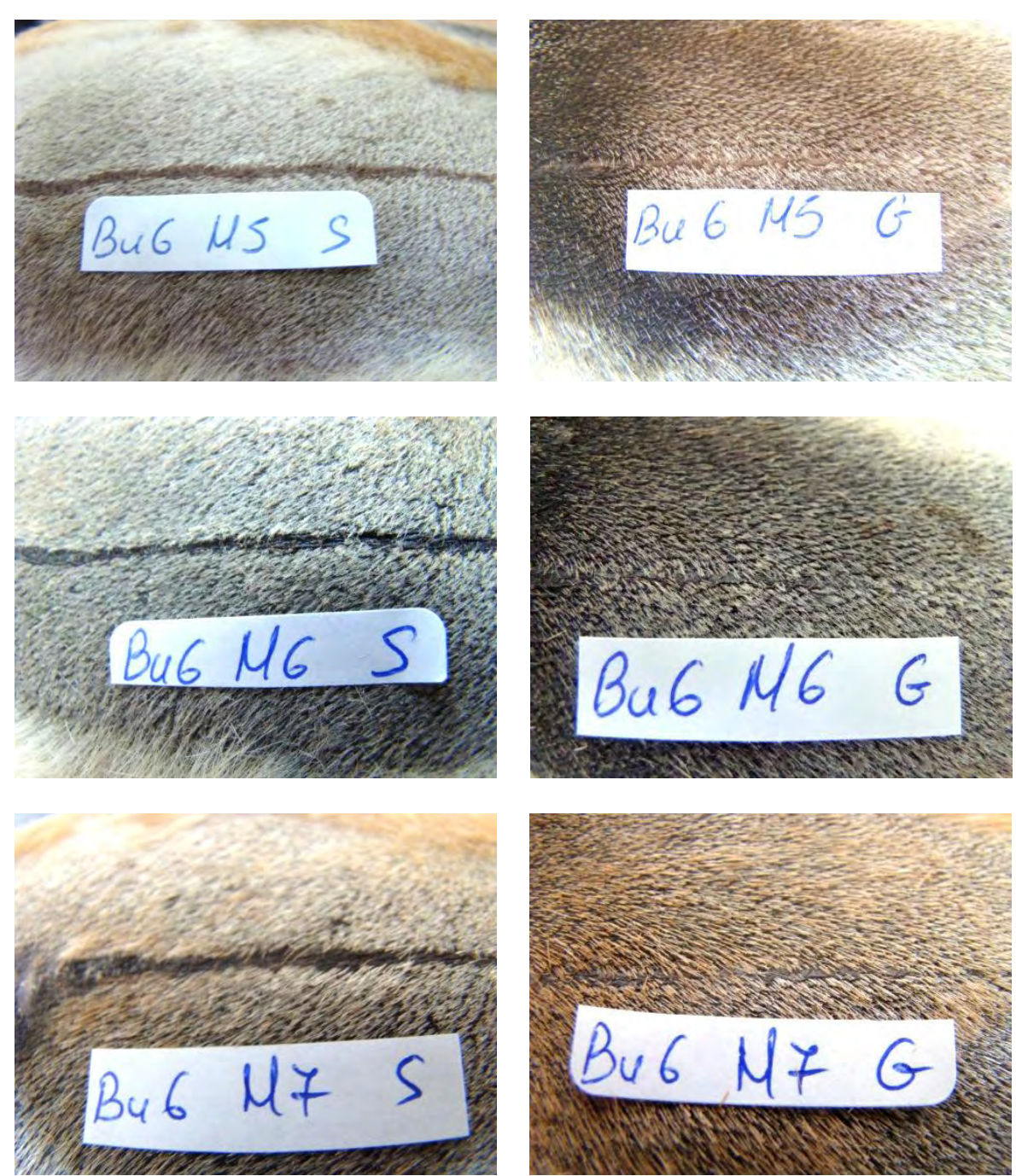
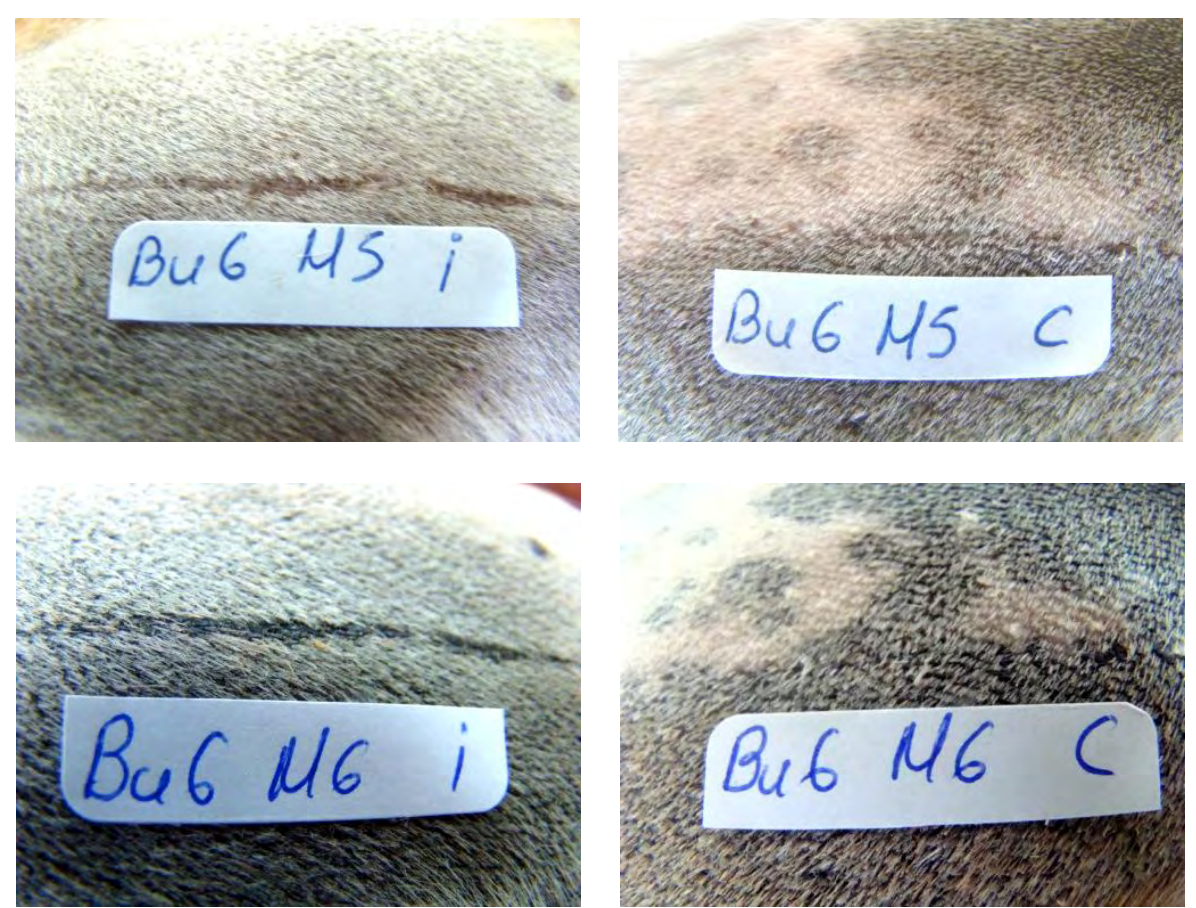

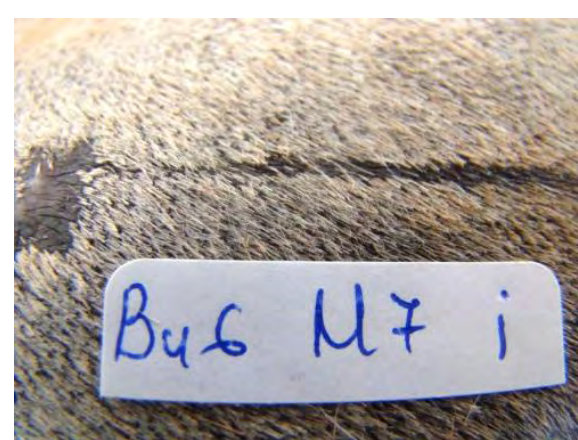

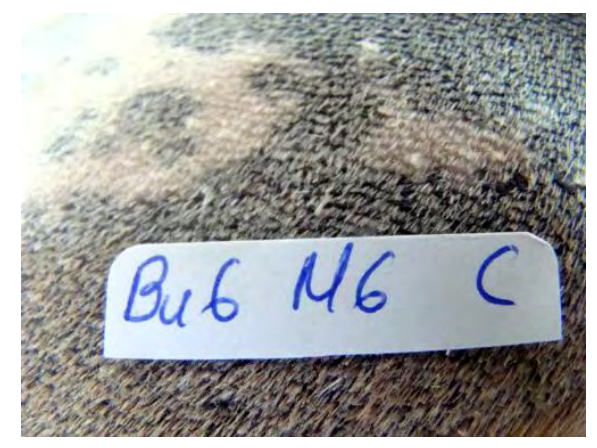

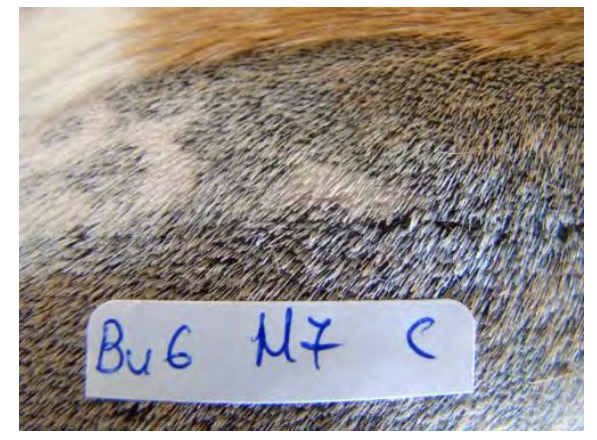


Beagle Bu6
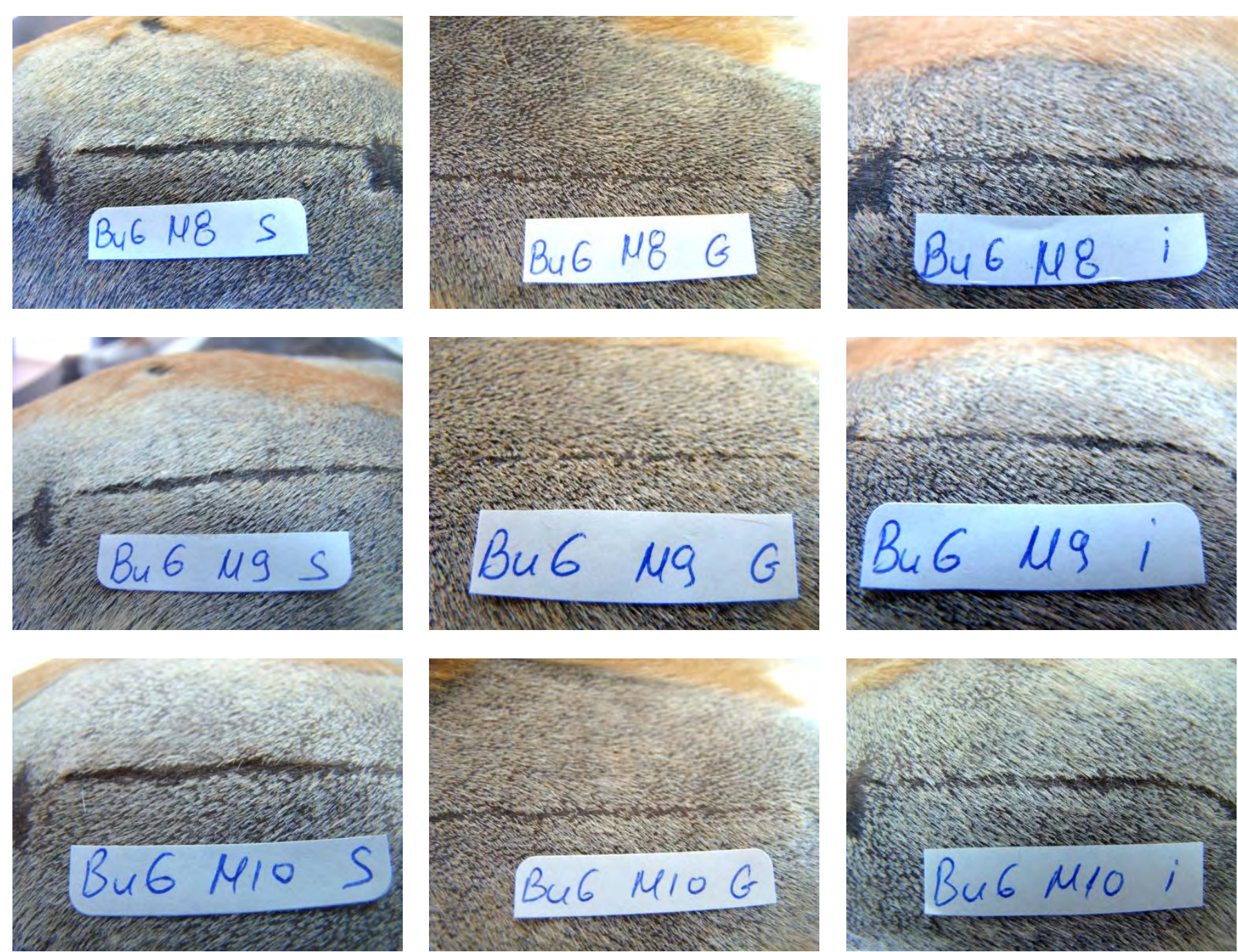
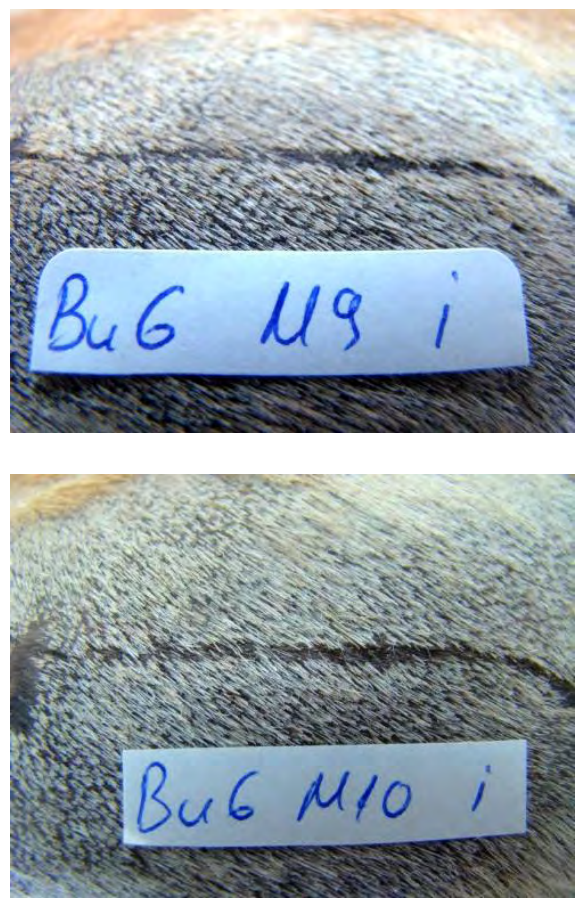
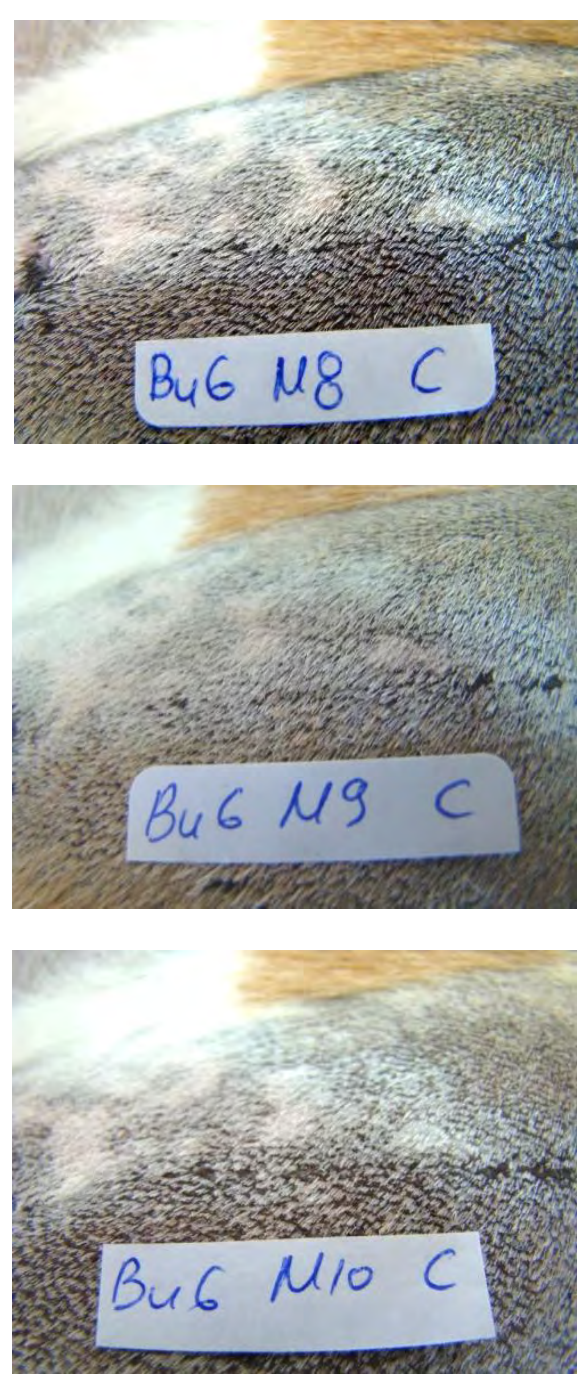


\section{Beagle Bu6}
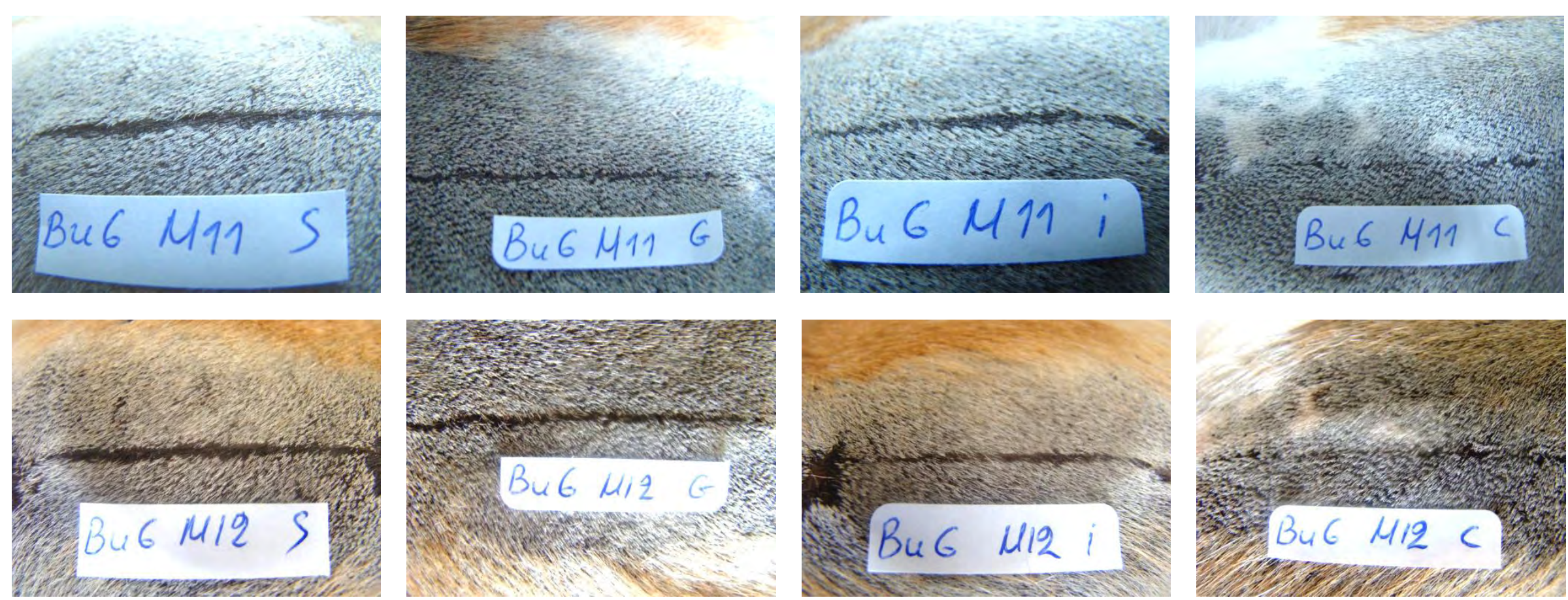
Beagle Bu7
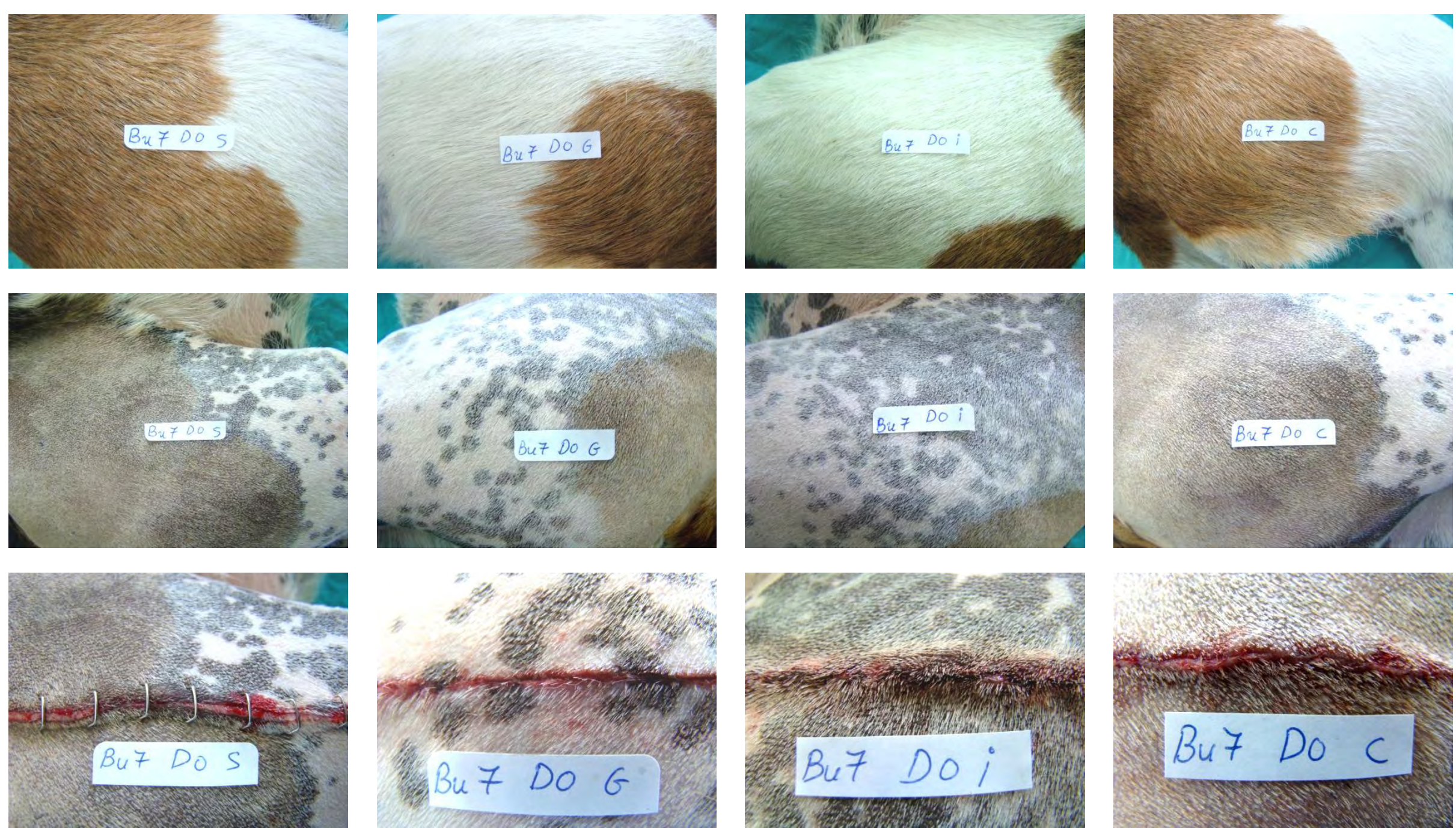

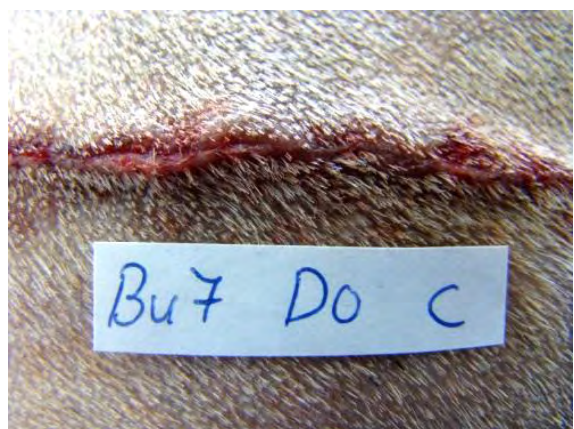




\section{Beagle Bu7}
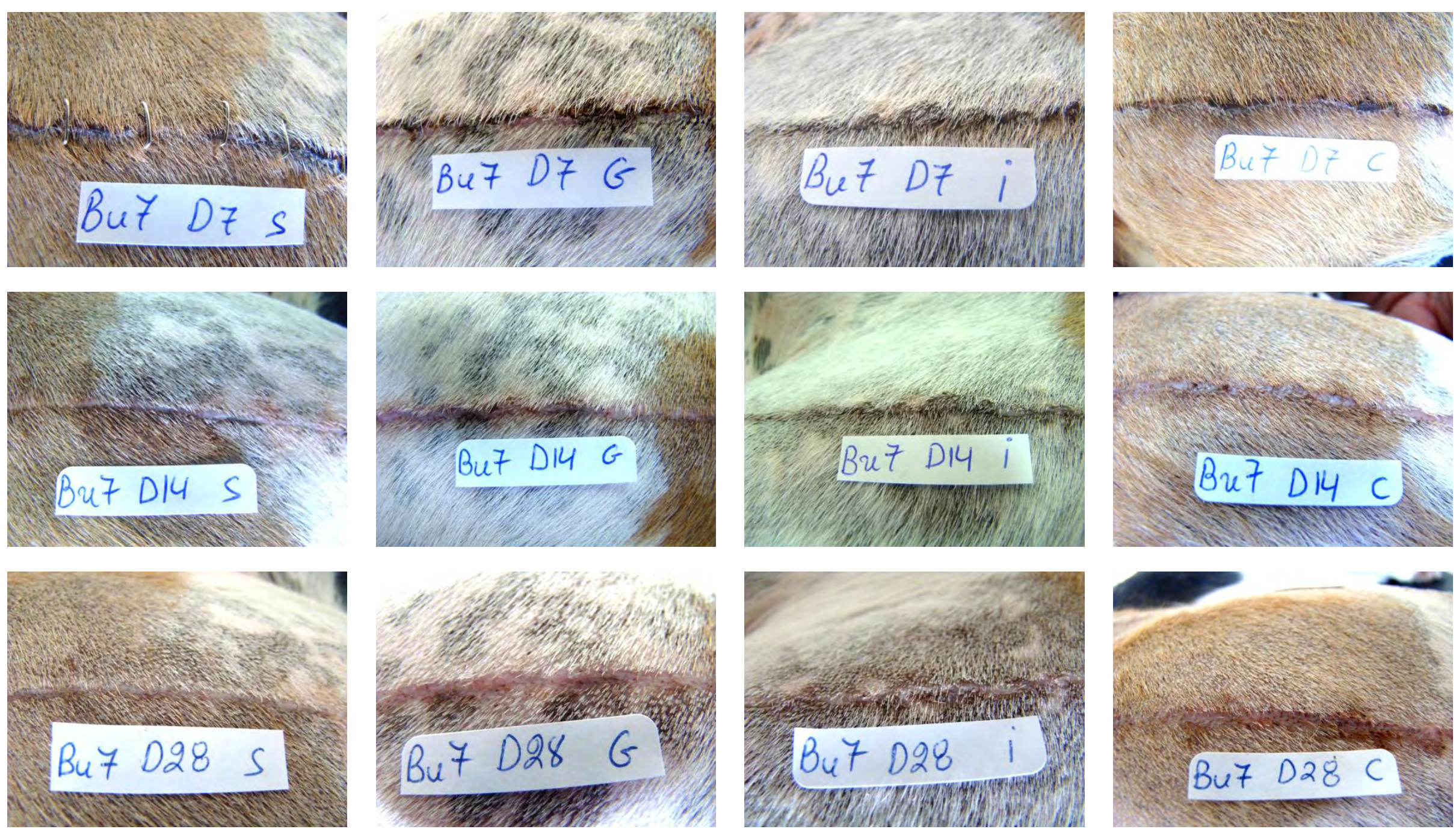
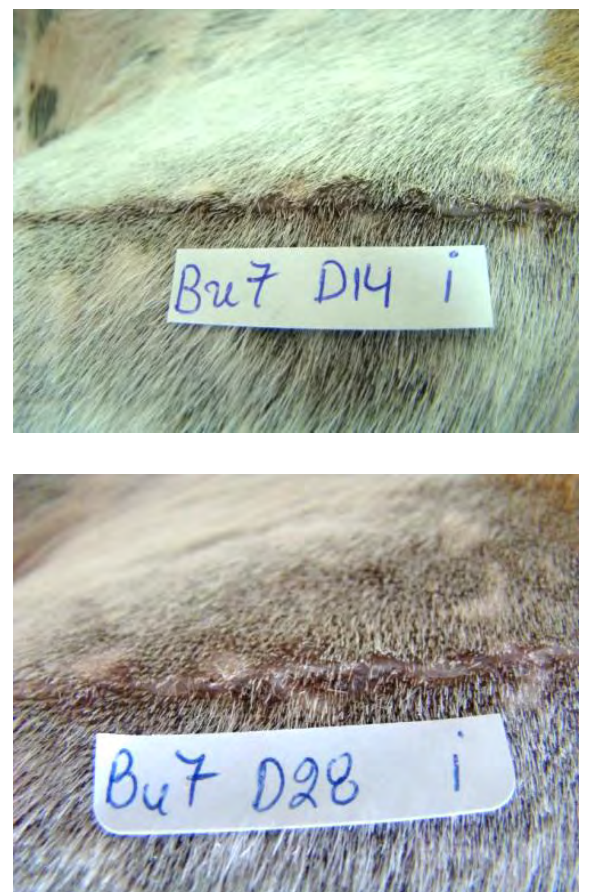
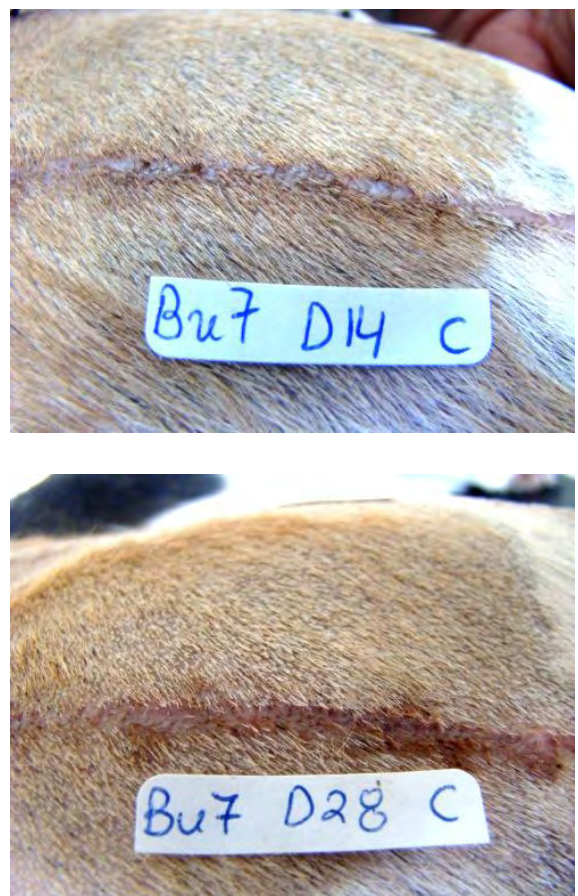
Beagle Bu7
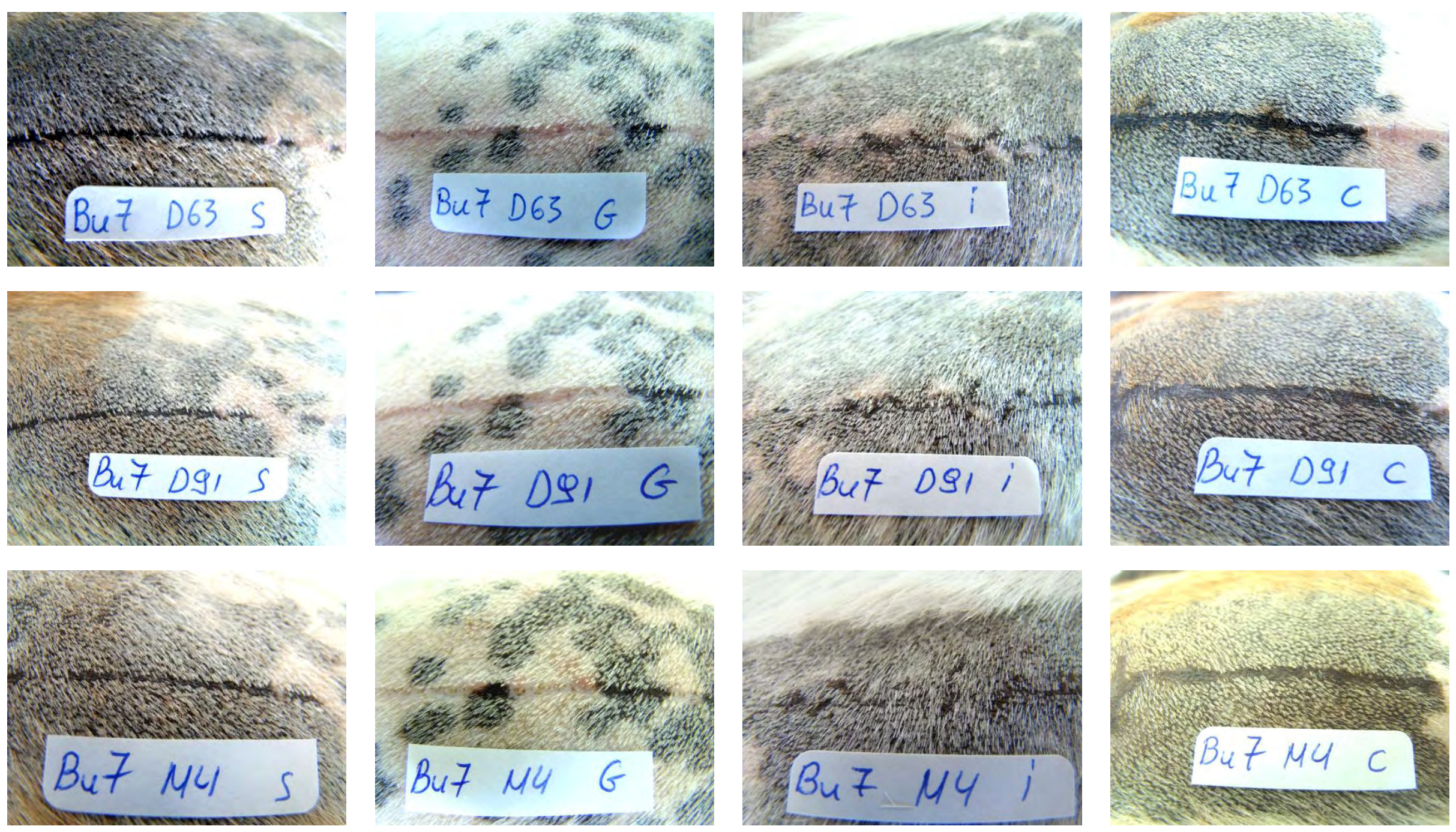


\section{Beagle Bu7}
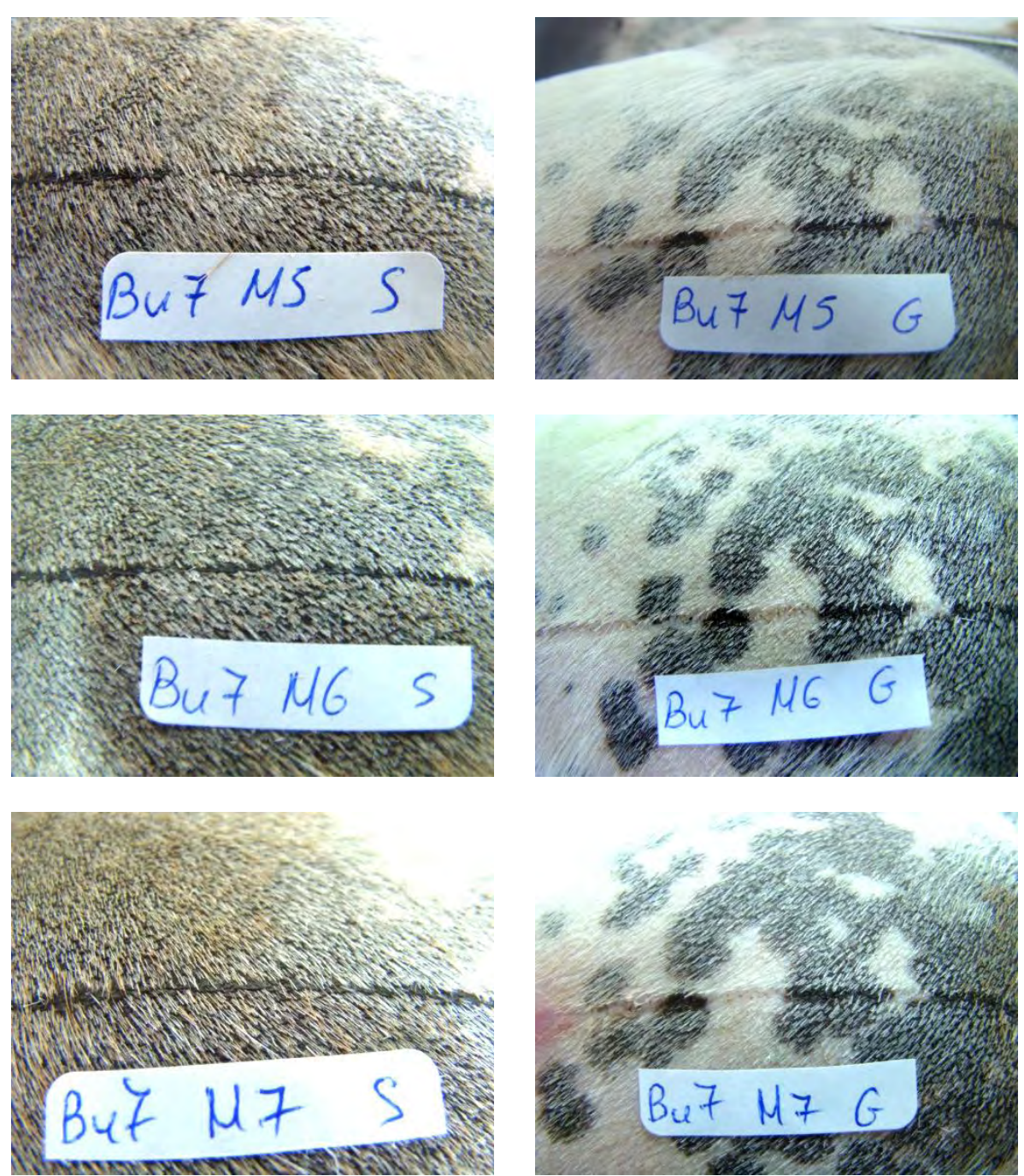
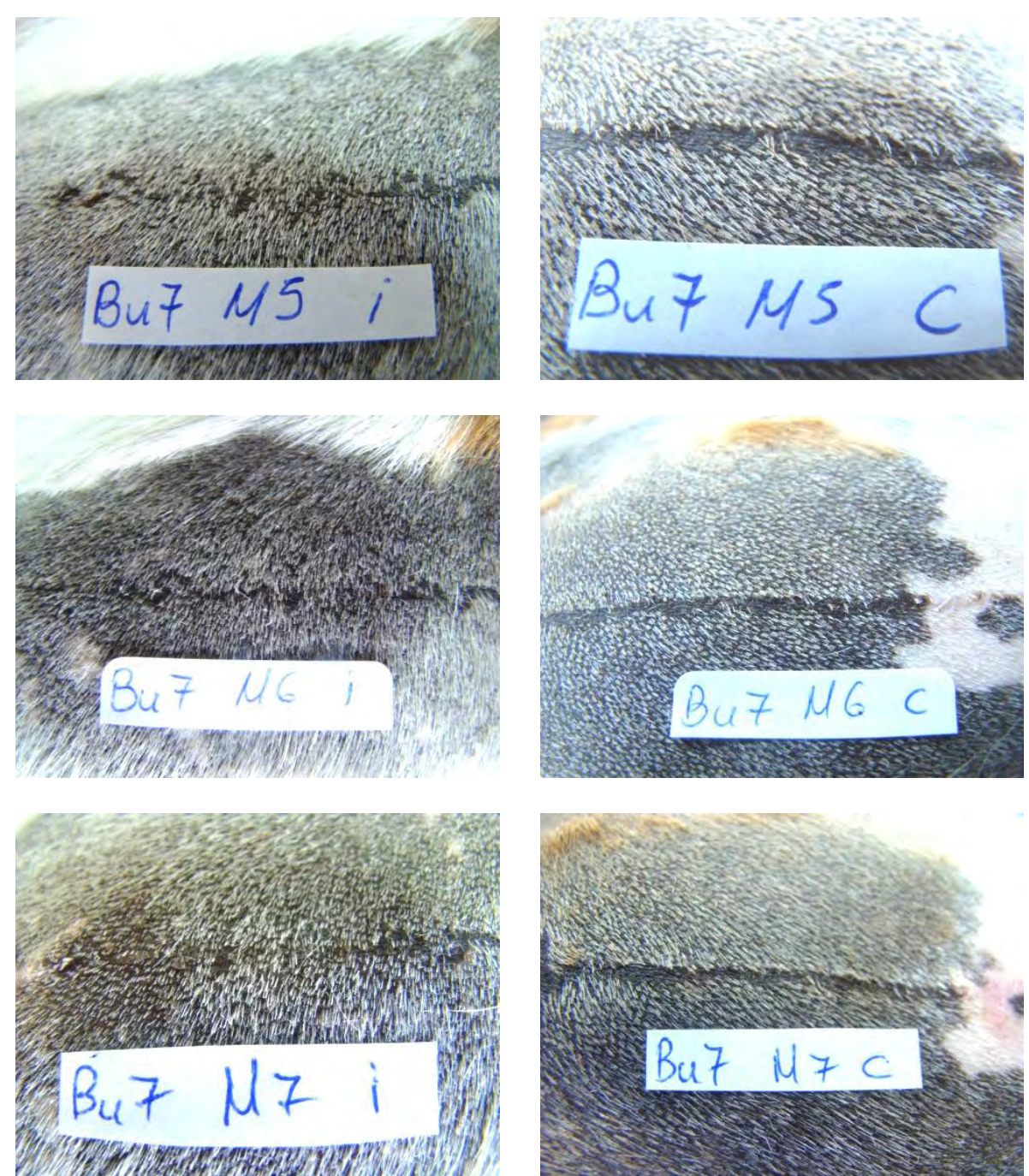
Beagle Bu7
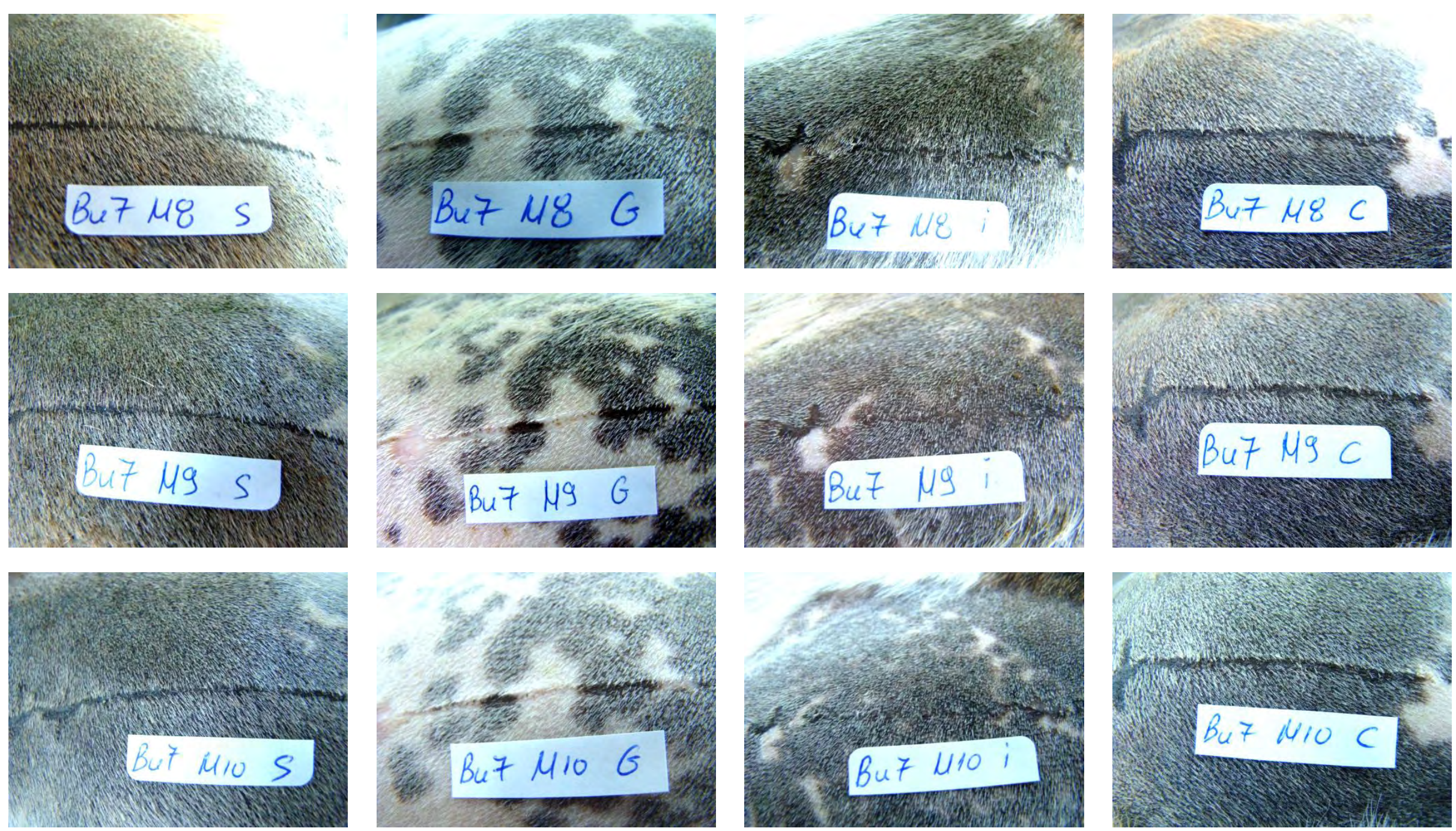

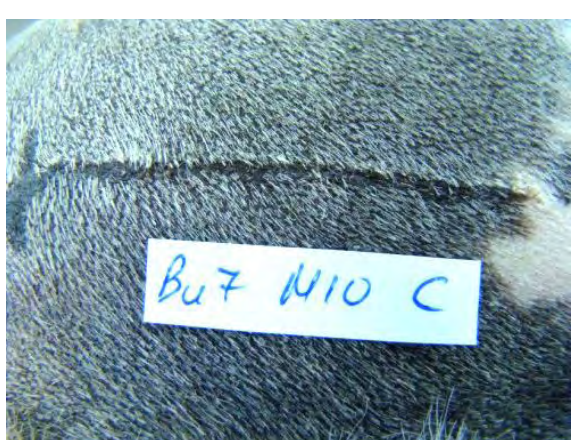


Beagle Bu7
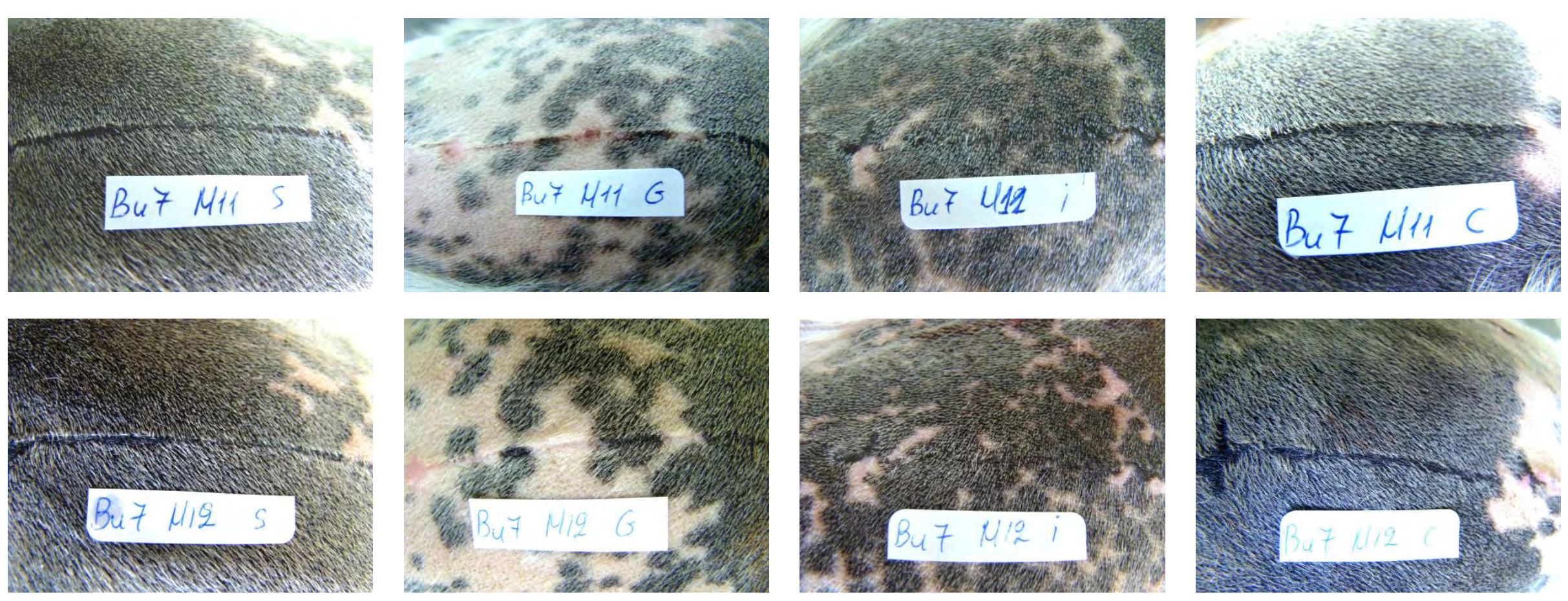
Beagle Bu8
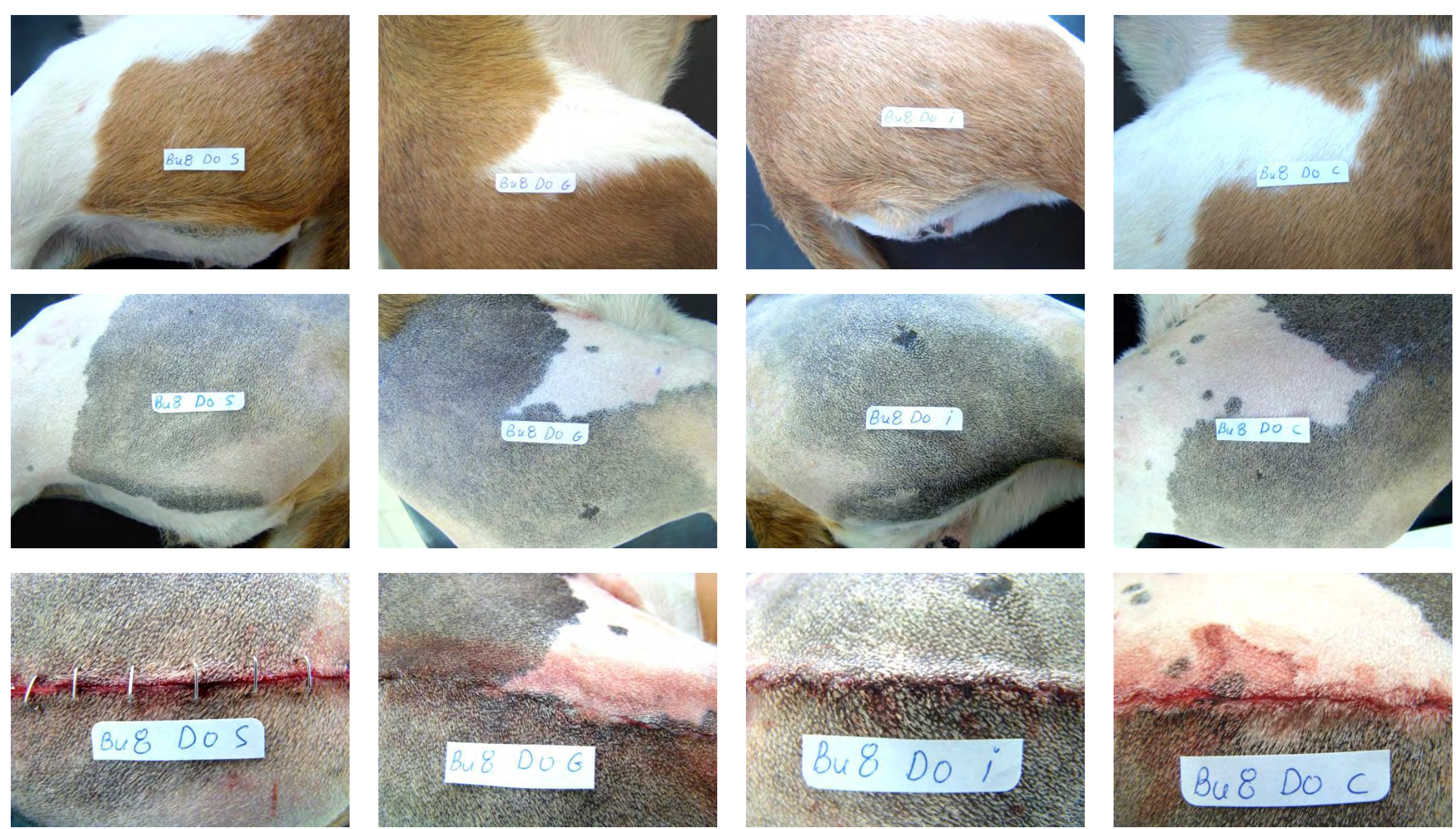


\section{Beagle Bu8}
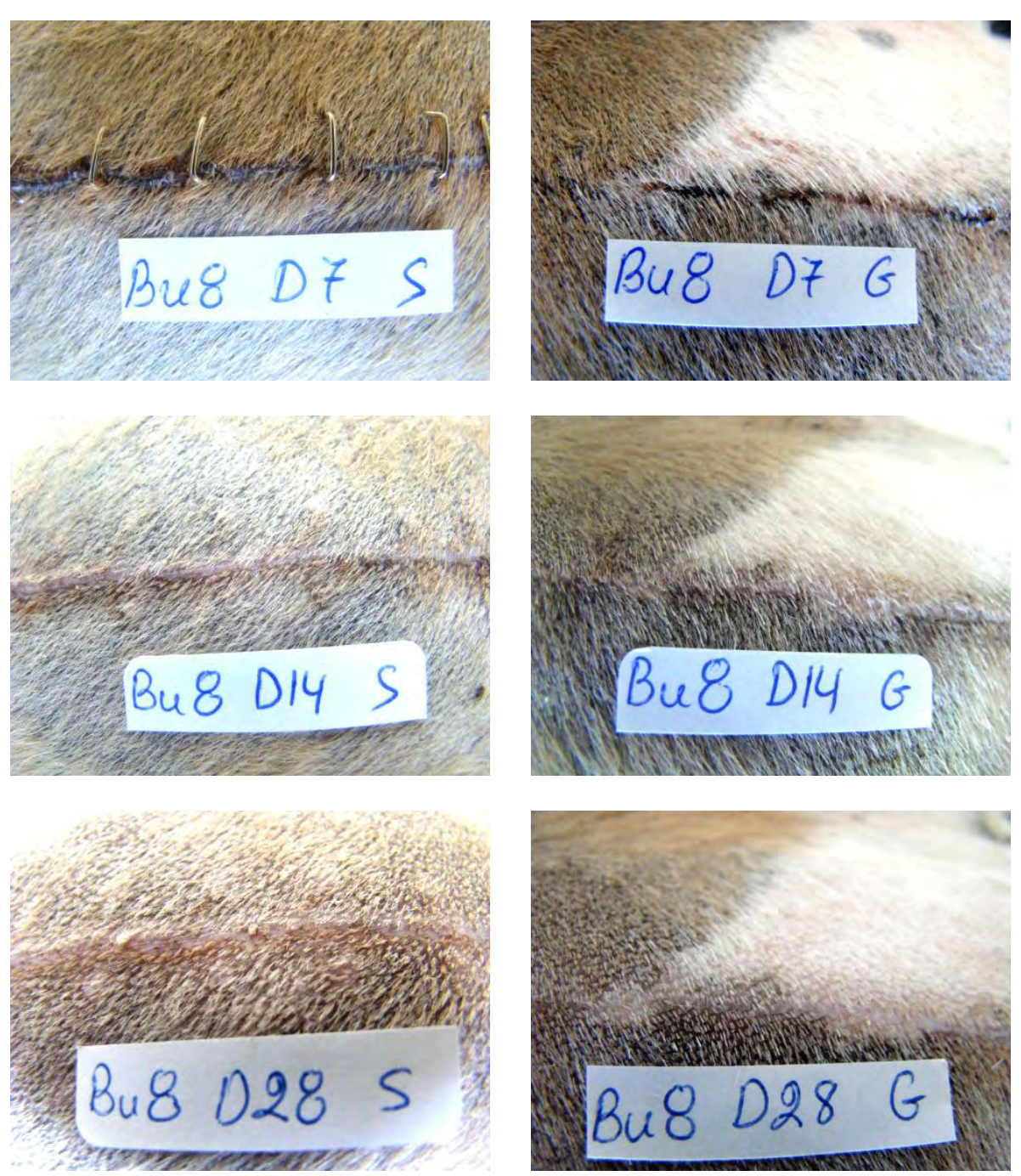
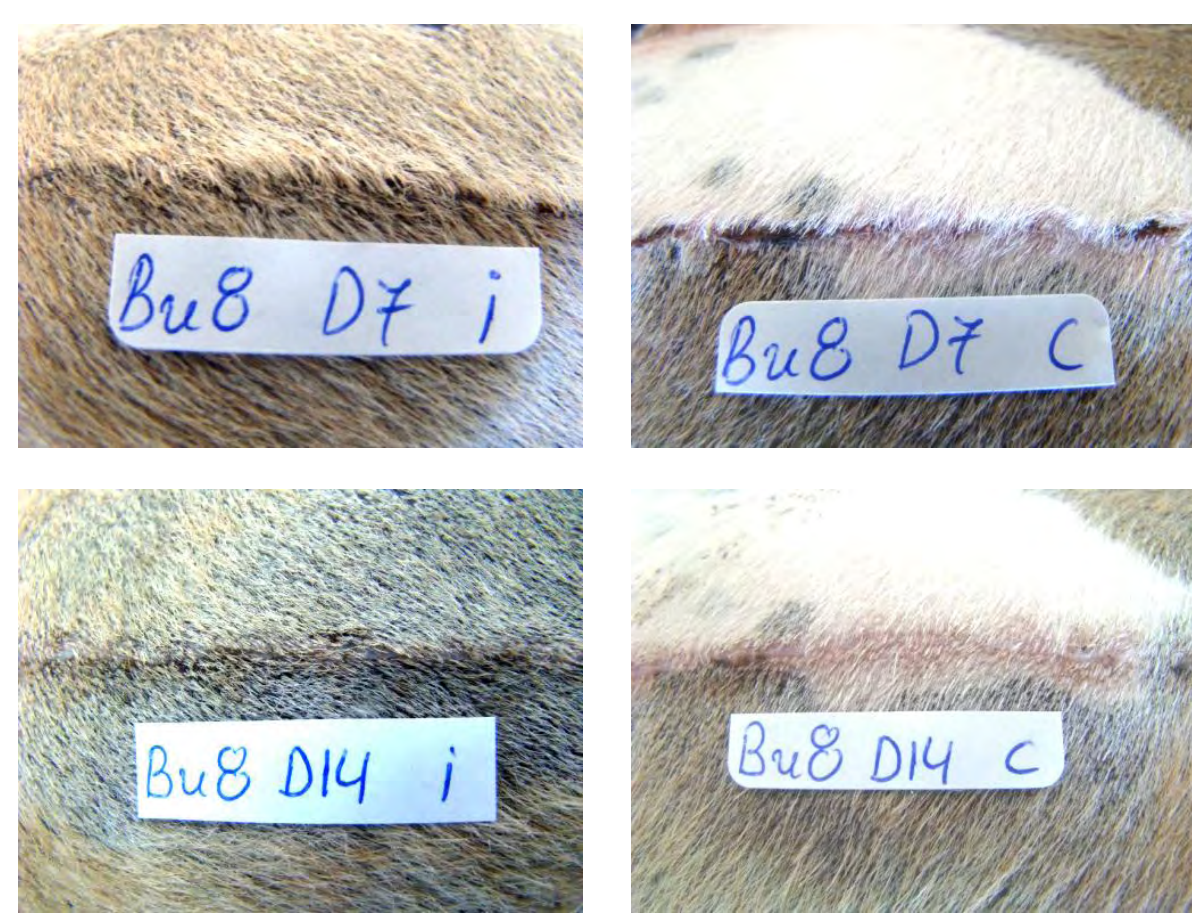
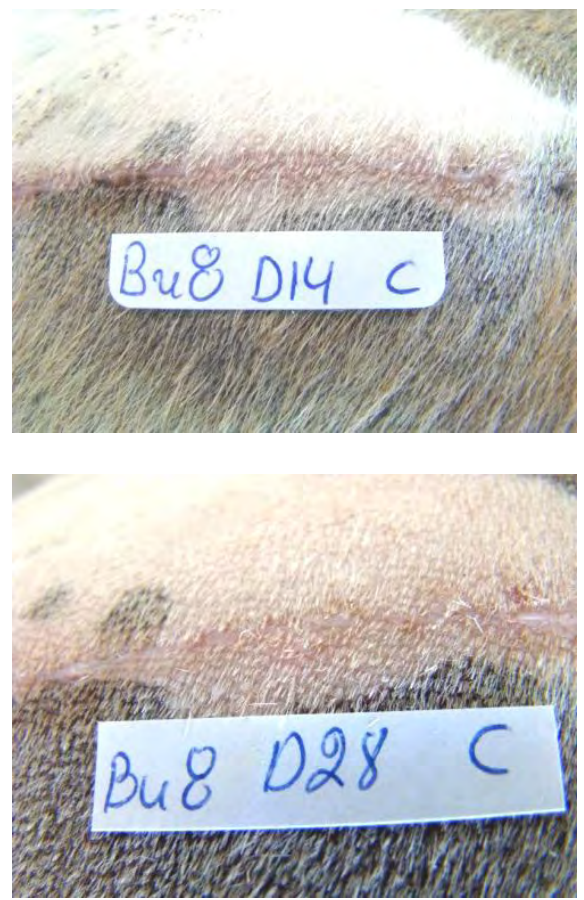
Beagle Bu8
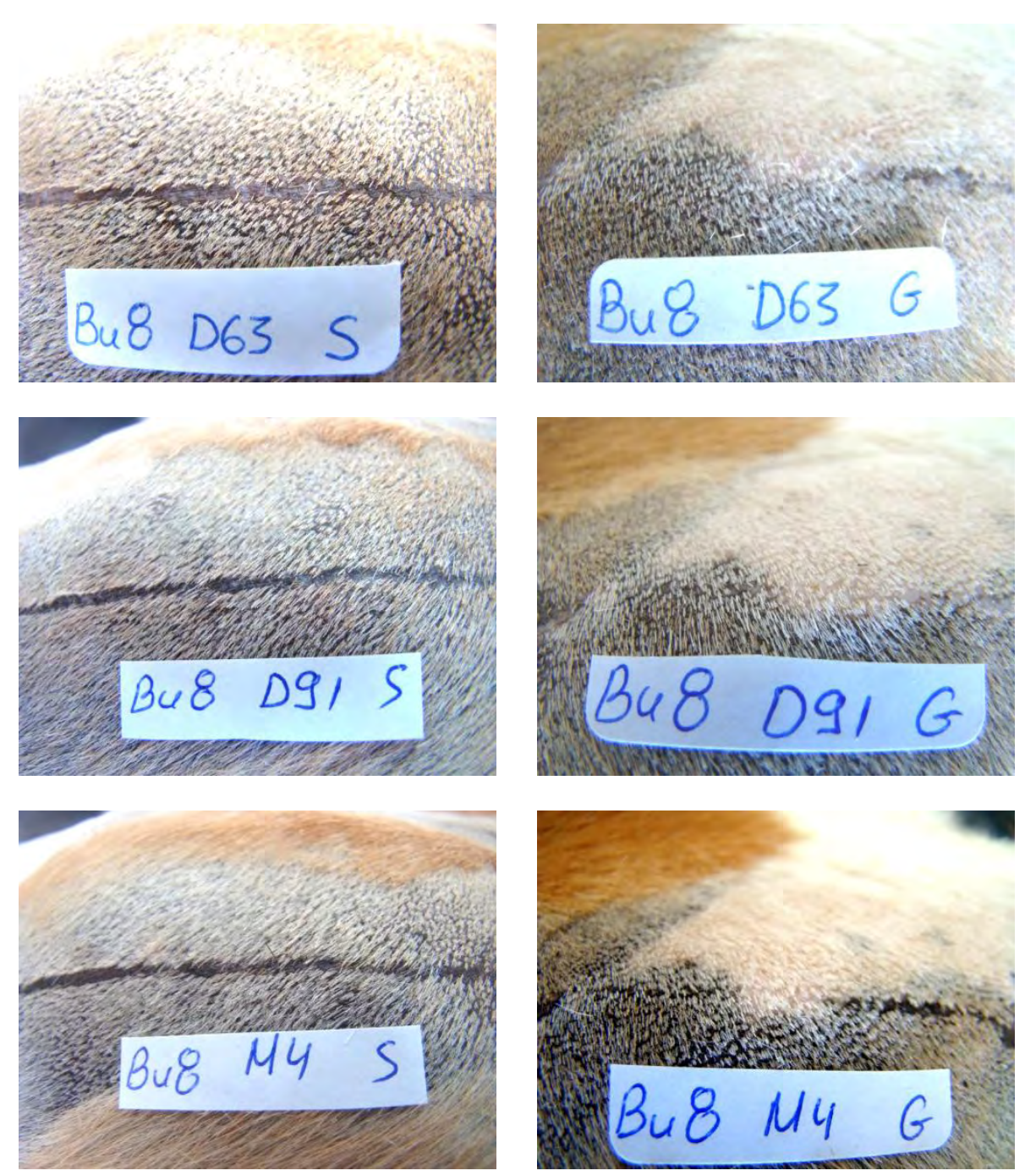
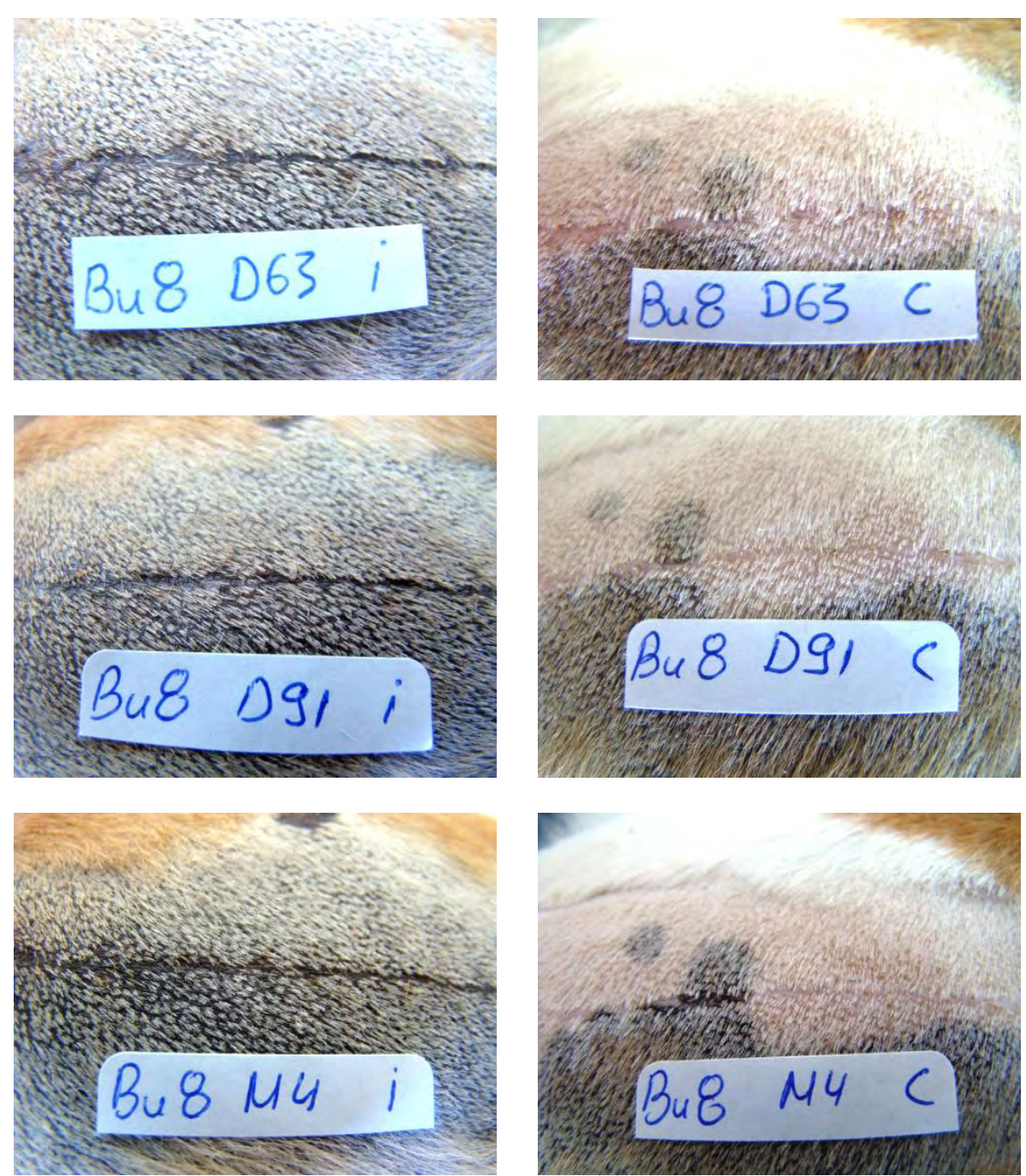


\section{Beagle Bu8}
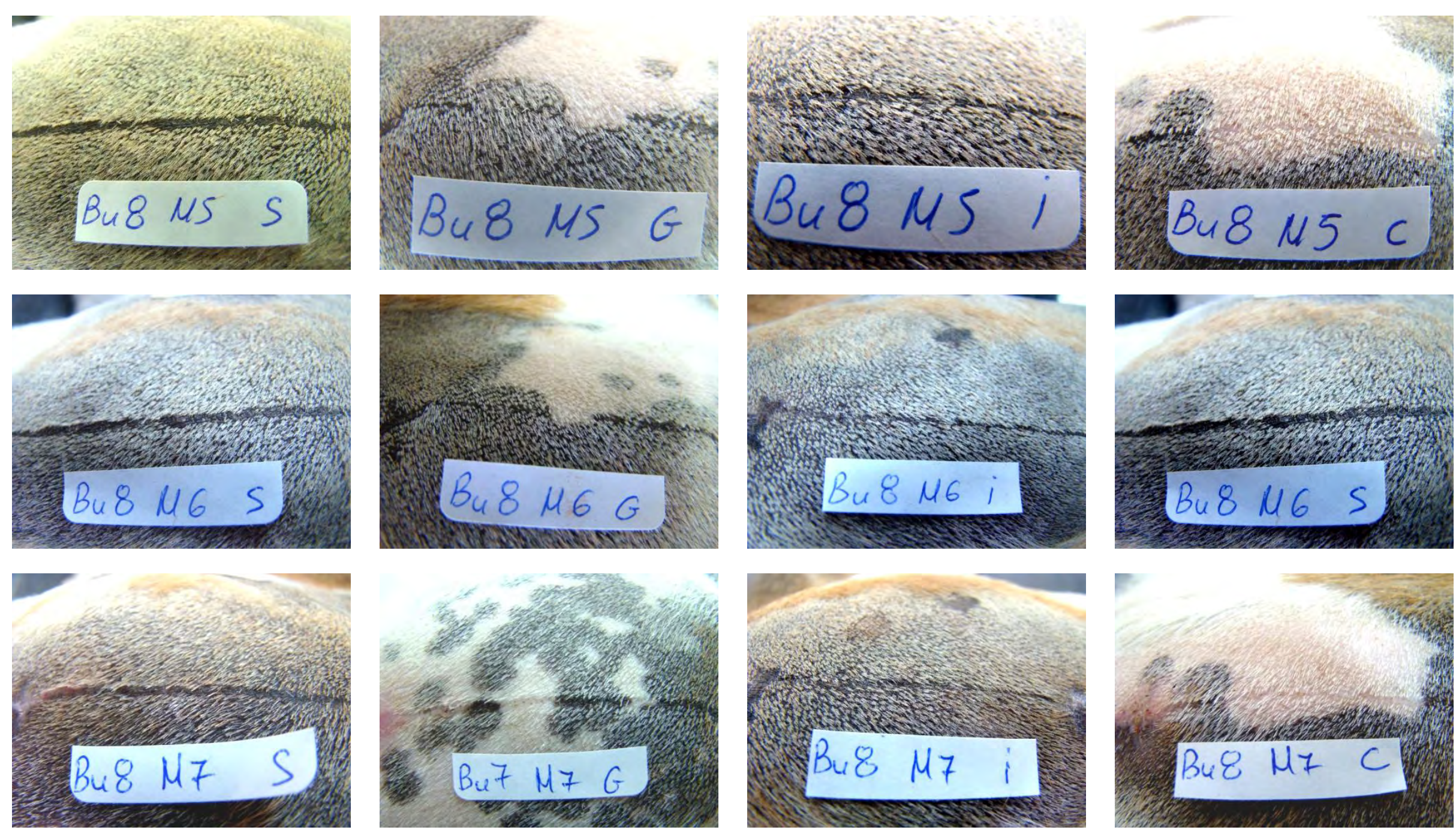


\section{Beagle Bu8}
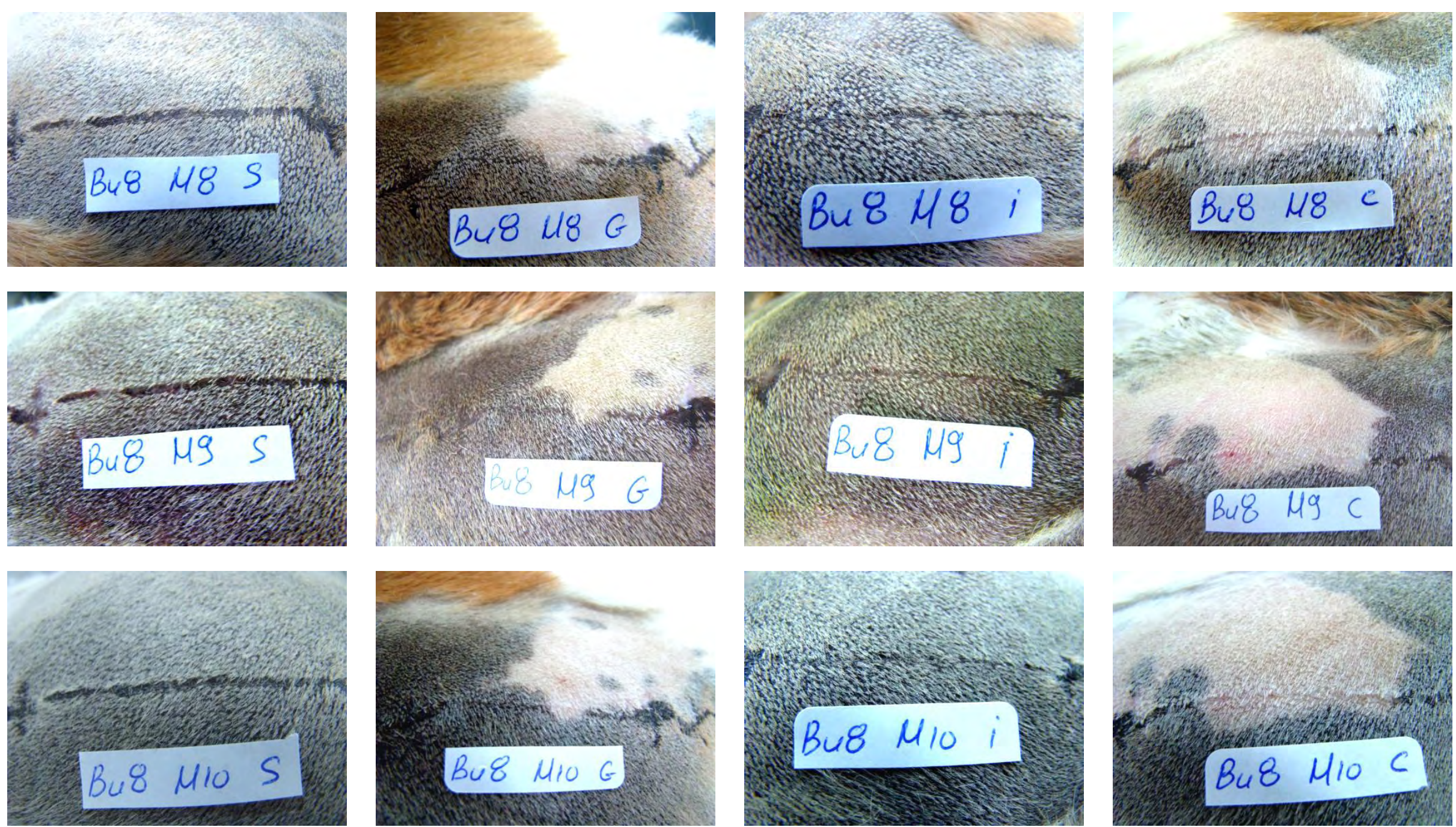


\section{Beagle Bu8}
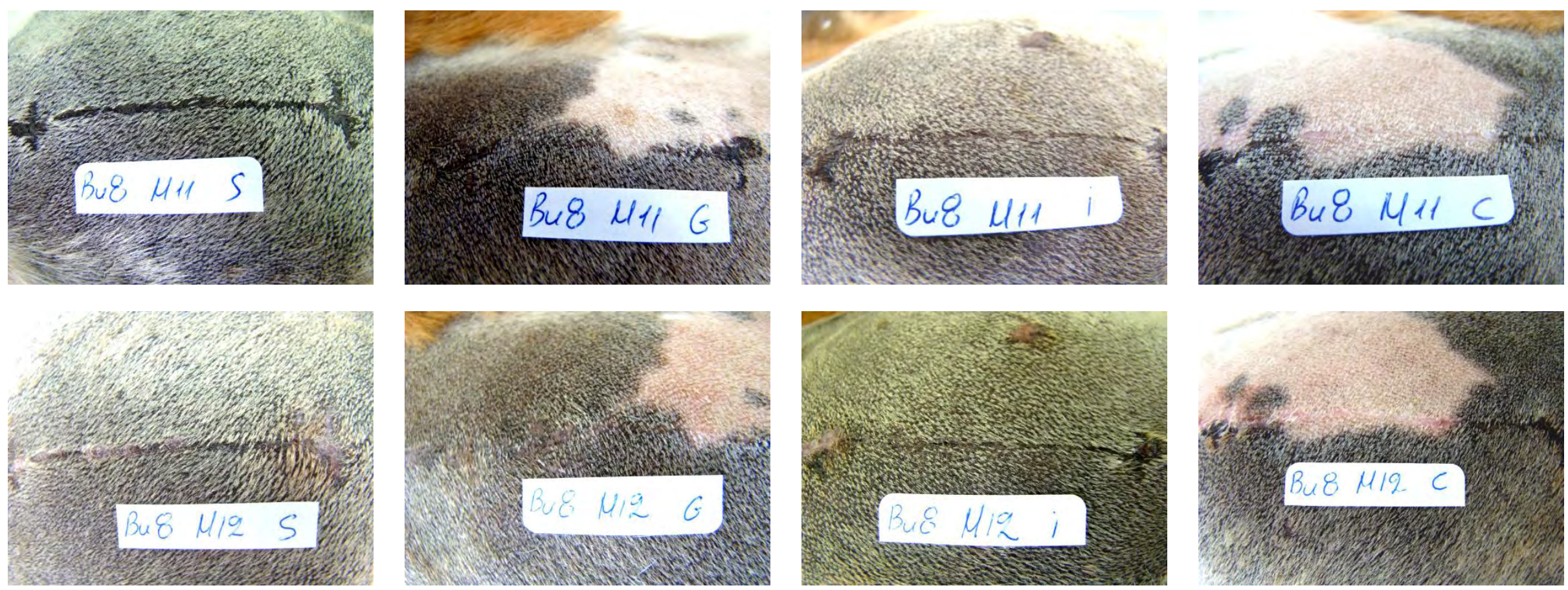


\section{Beagle Bu9}
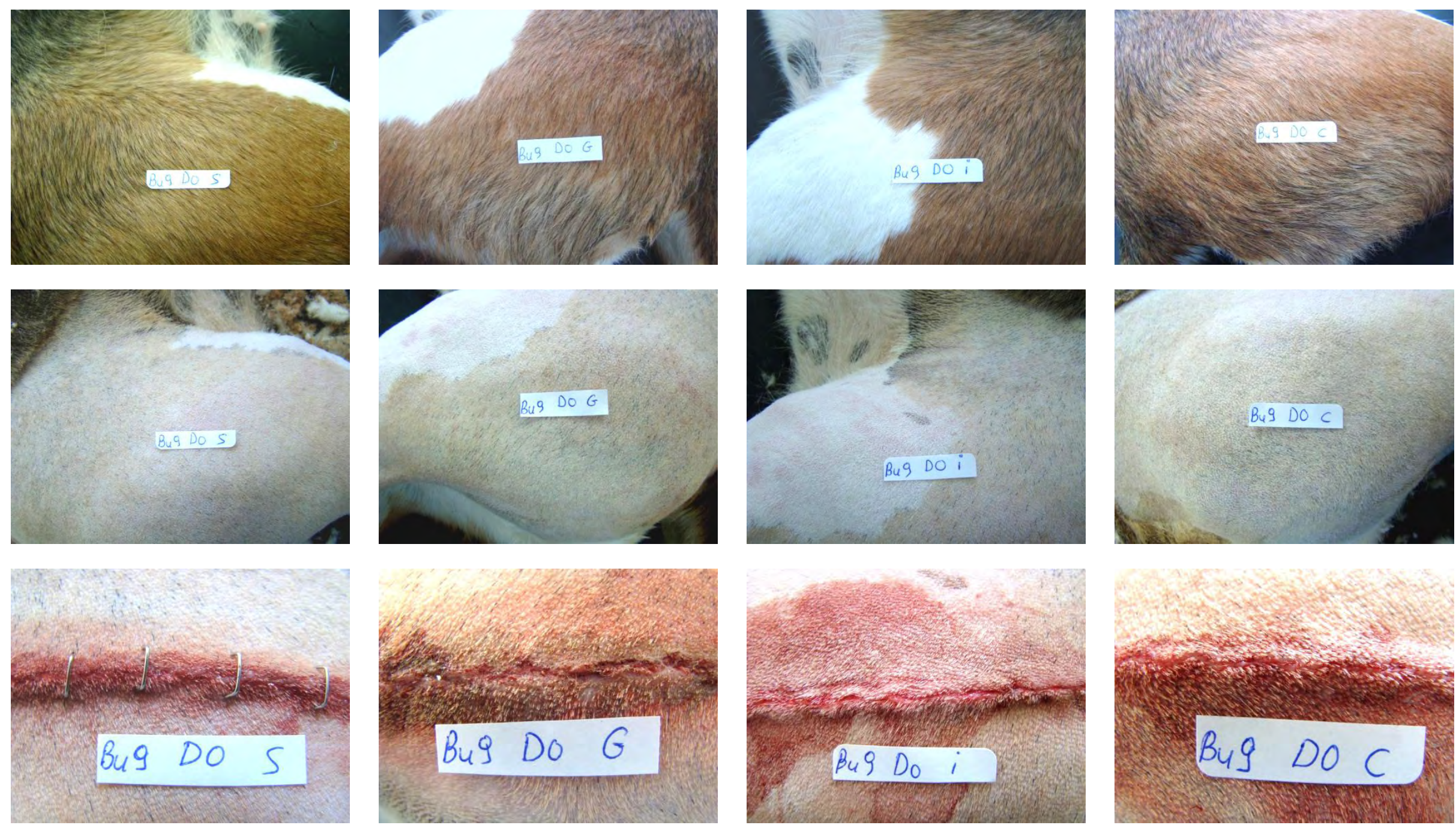


\section{Beagle Bu9}
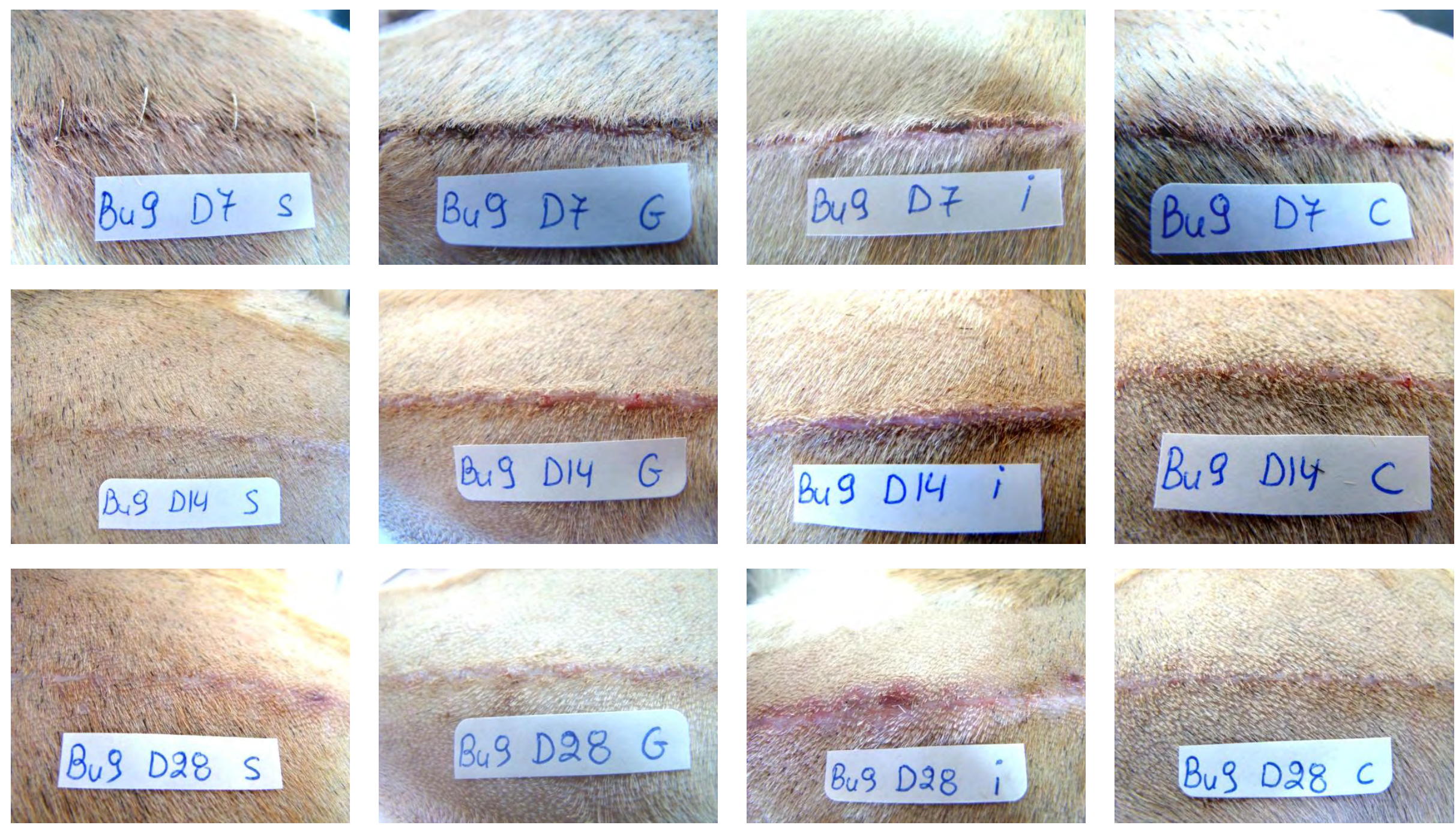


\section{Beagle Bu9}
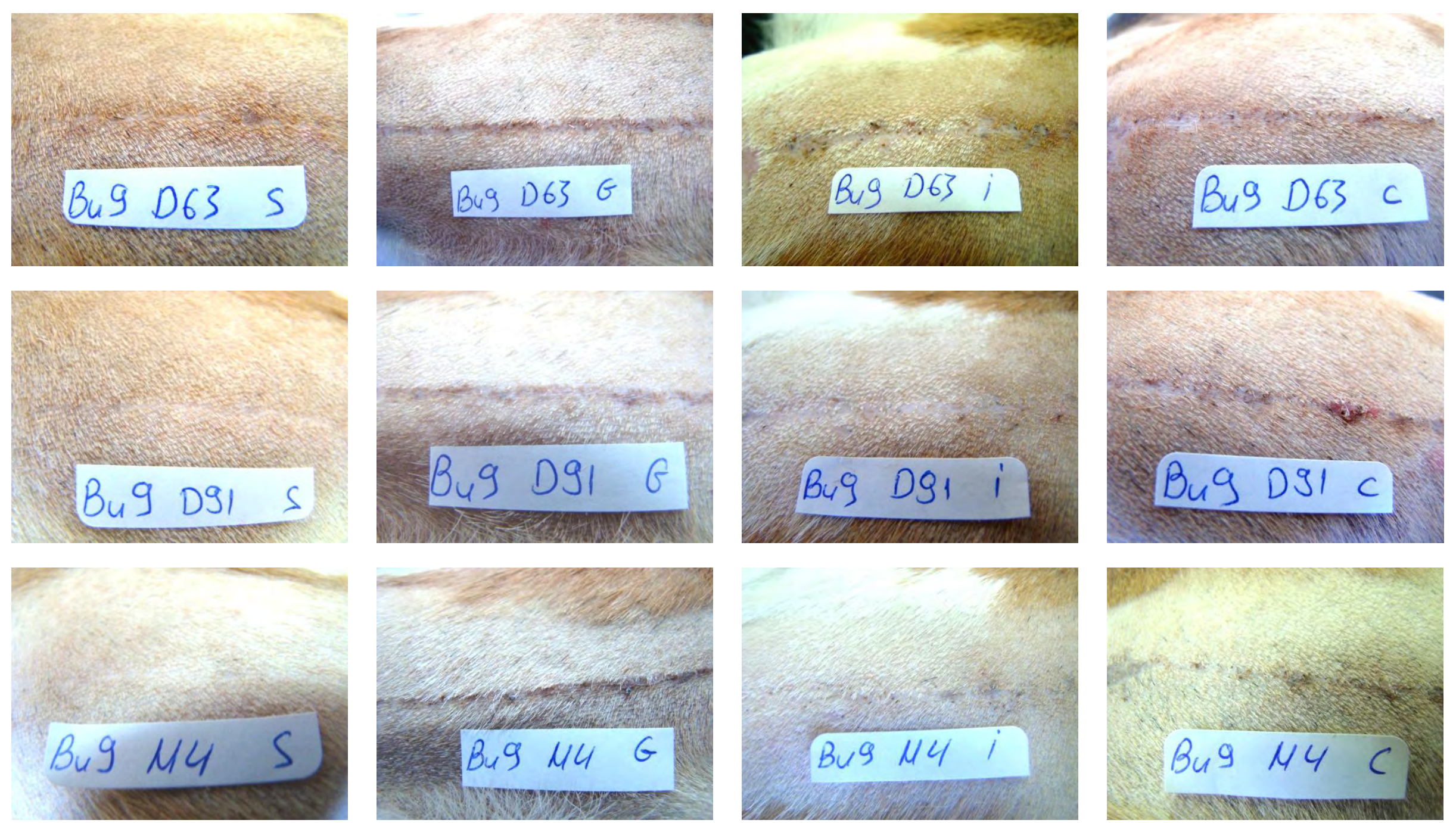


\section{Beagle Bu9}
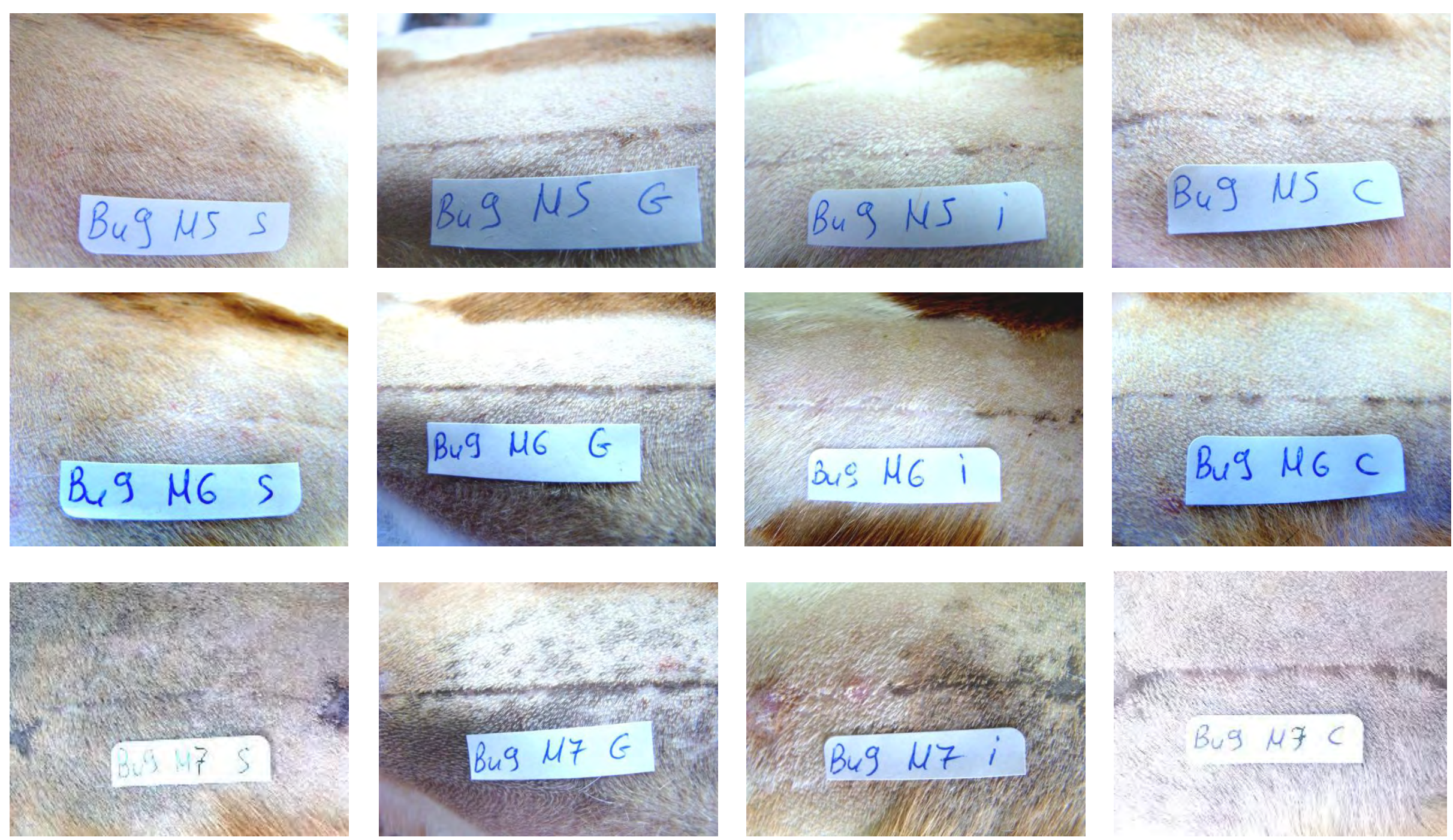


\section{Beagle Bu9}
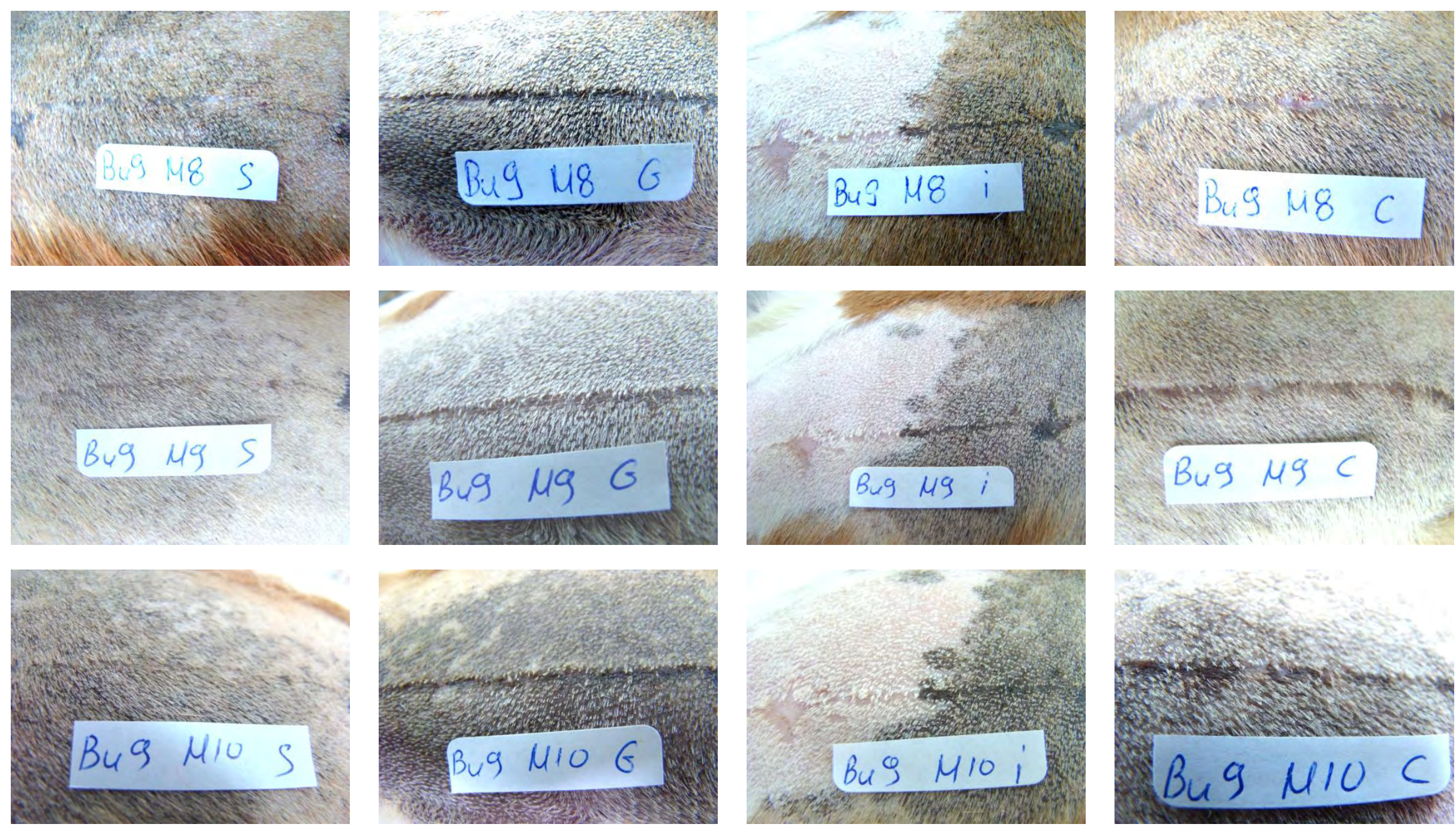


\section{Beagle Bu9}
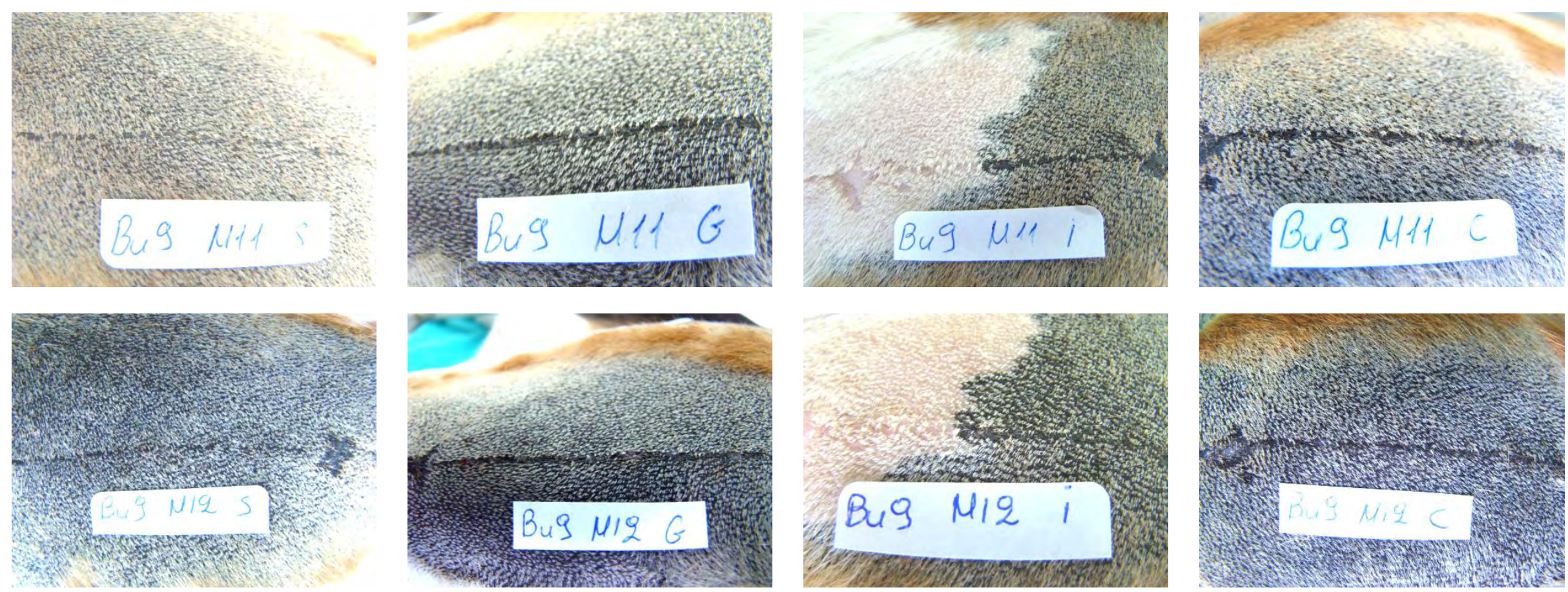


\section{Beagle Bu10}
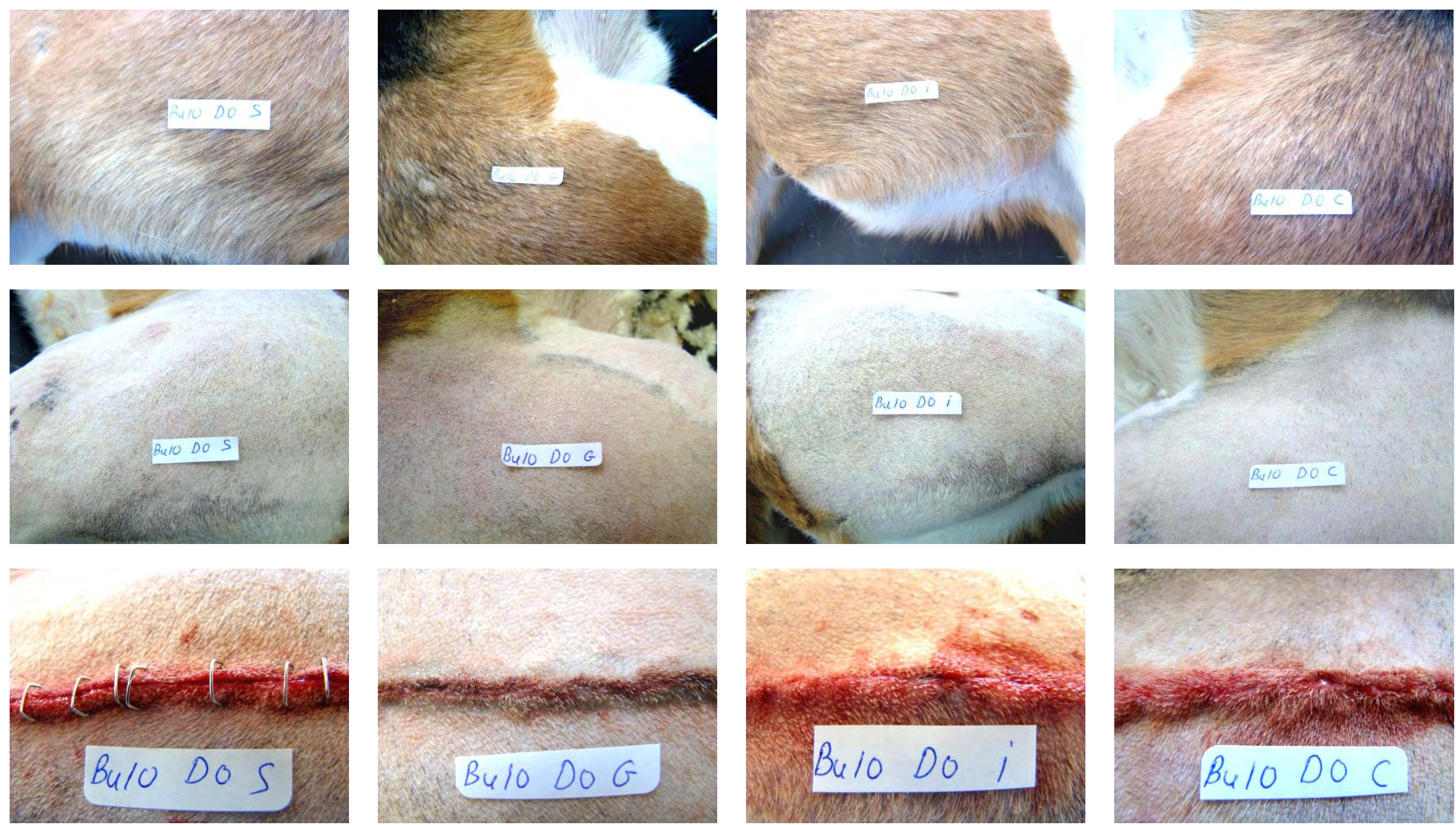


\section{Beagle Bu10}
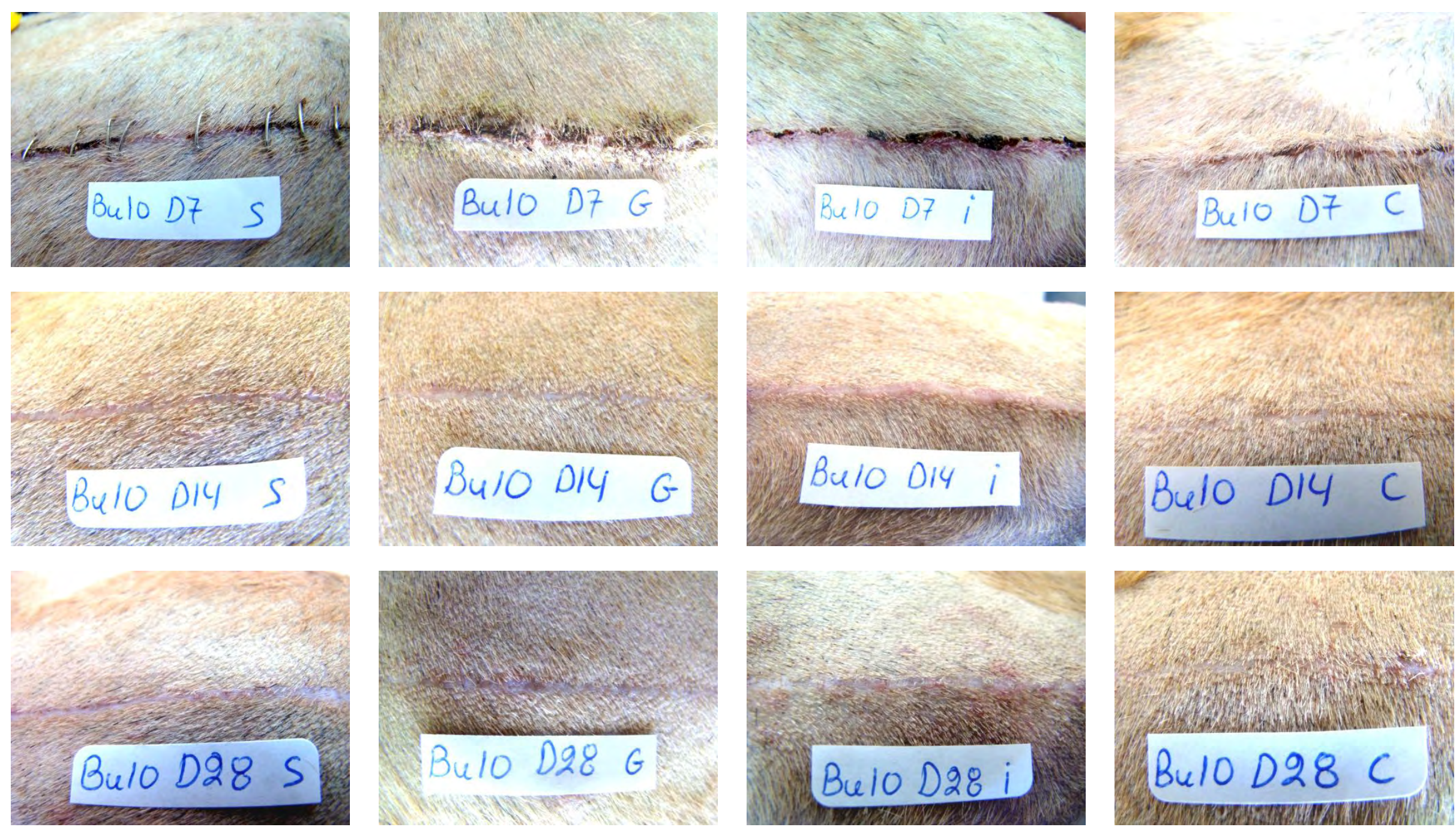


\section{Beagle Bu10}
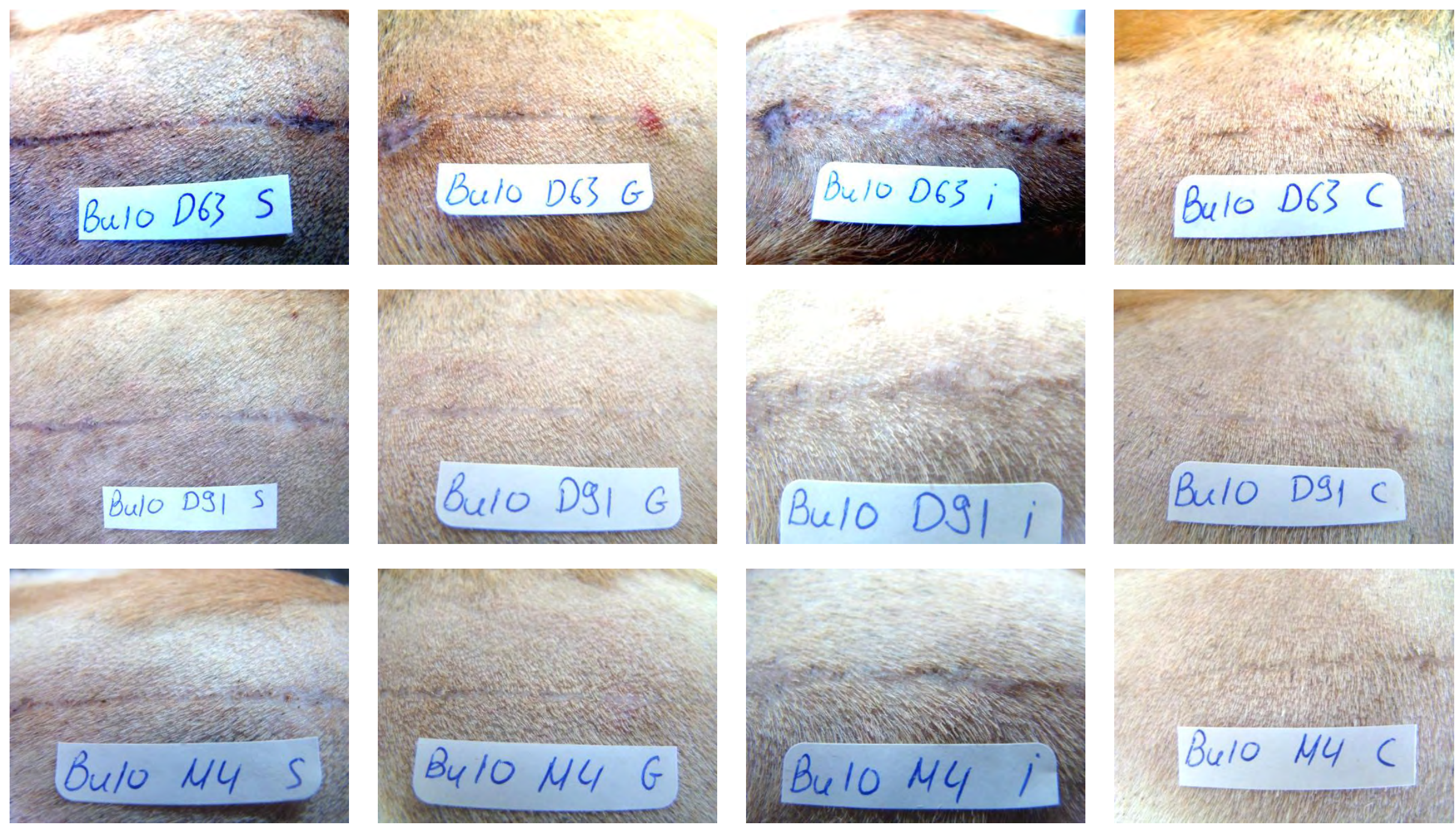


\section{Beagle Bu10}
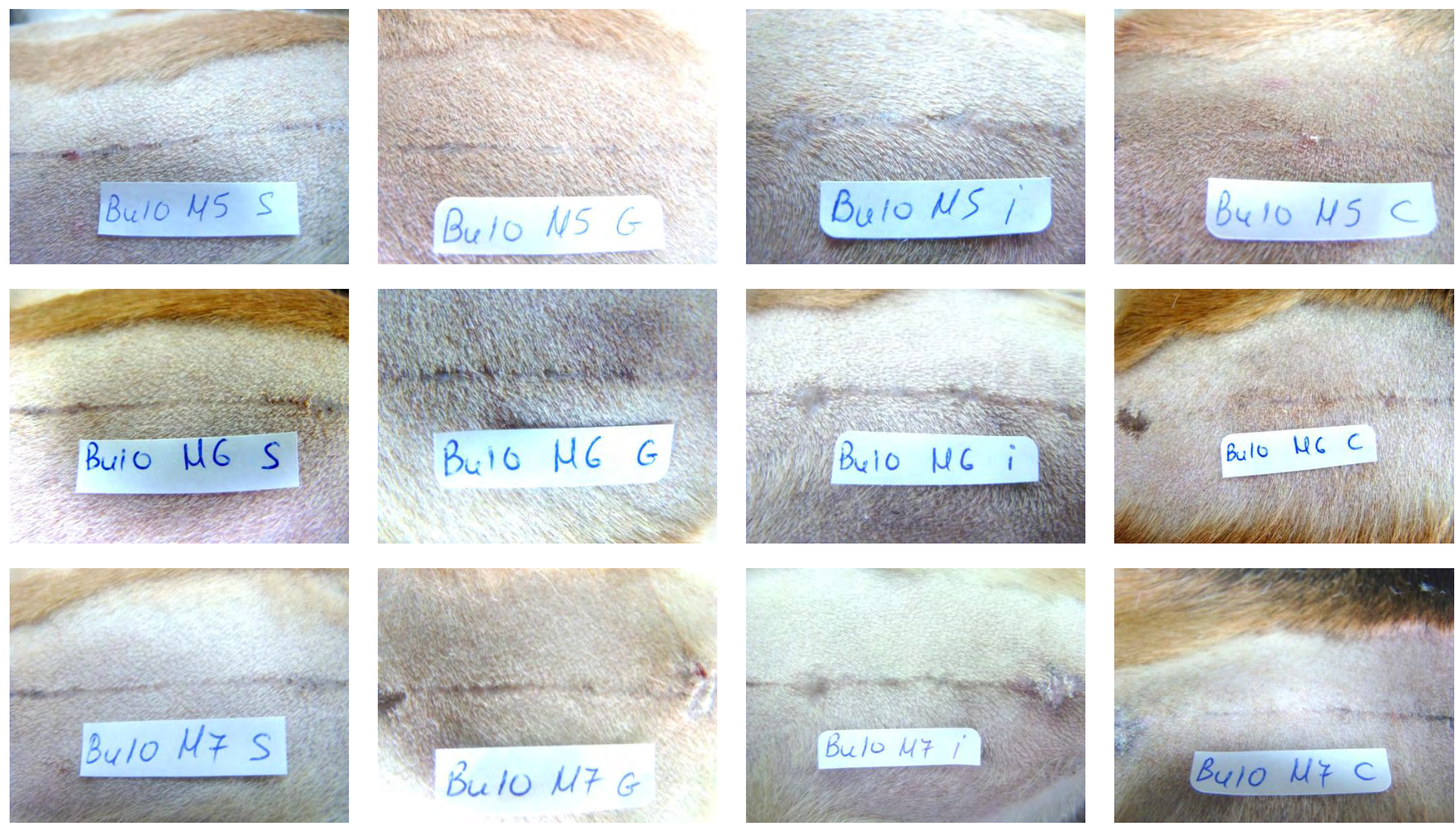


\section{Beagle Bu10}
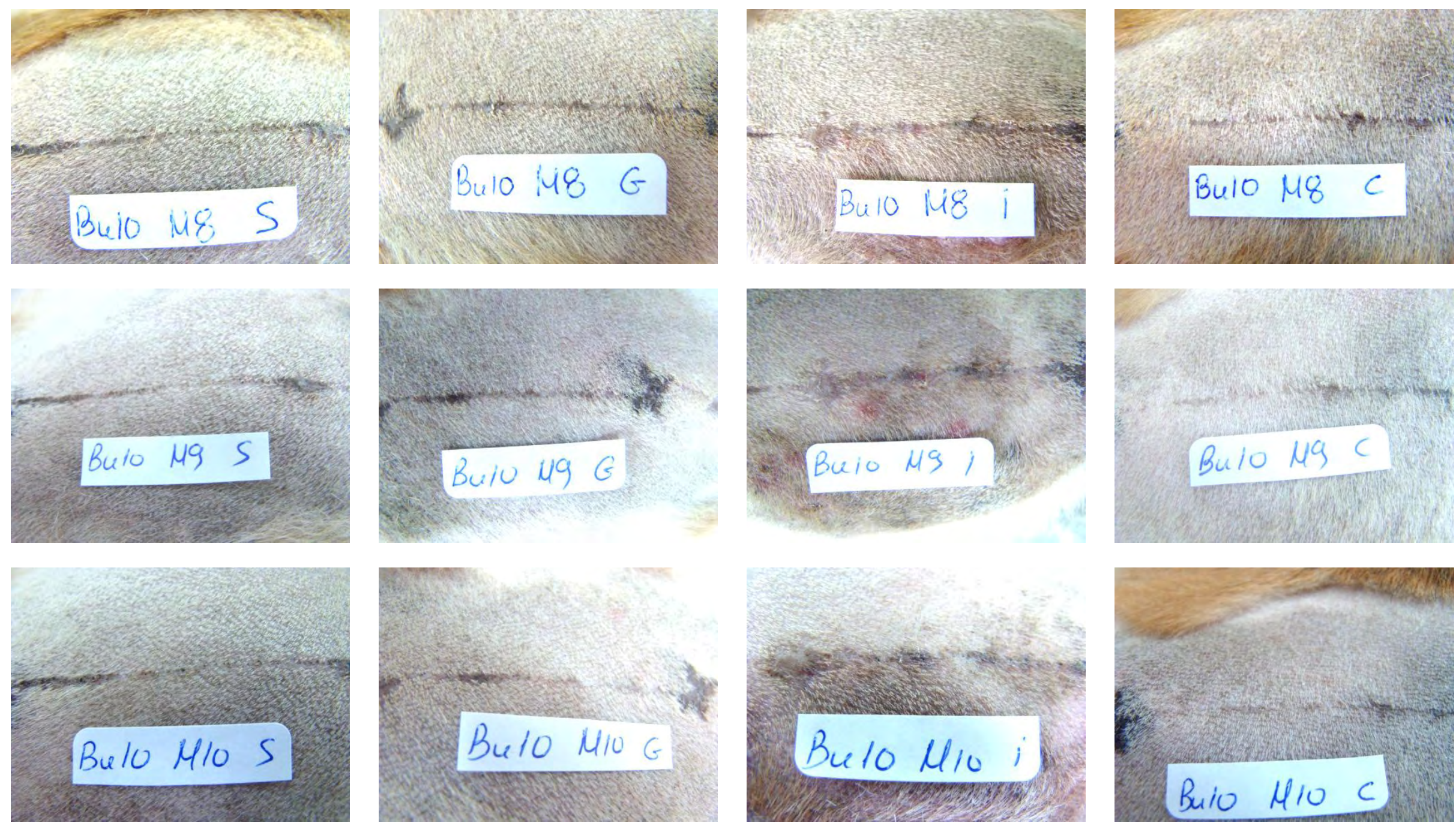


\section{Beagle Bu10}
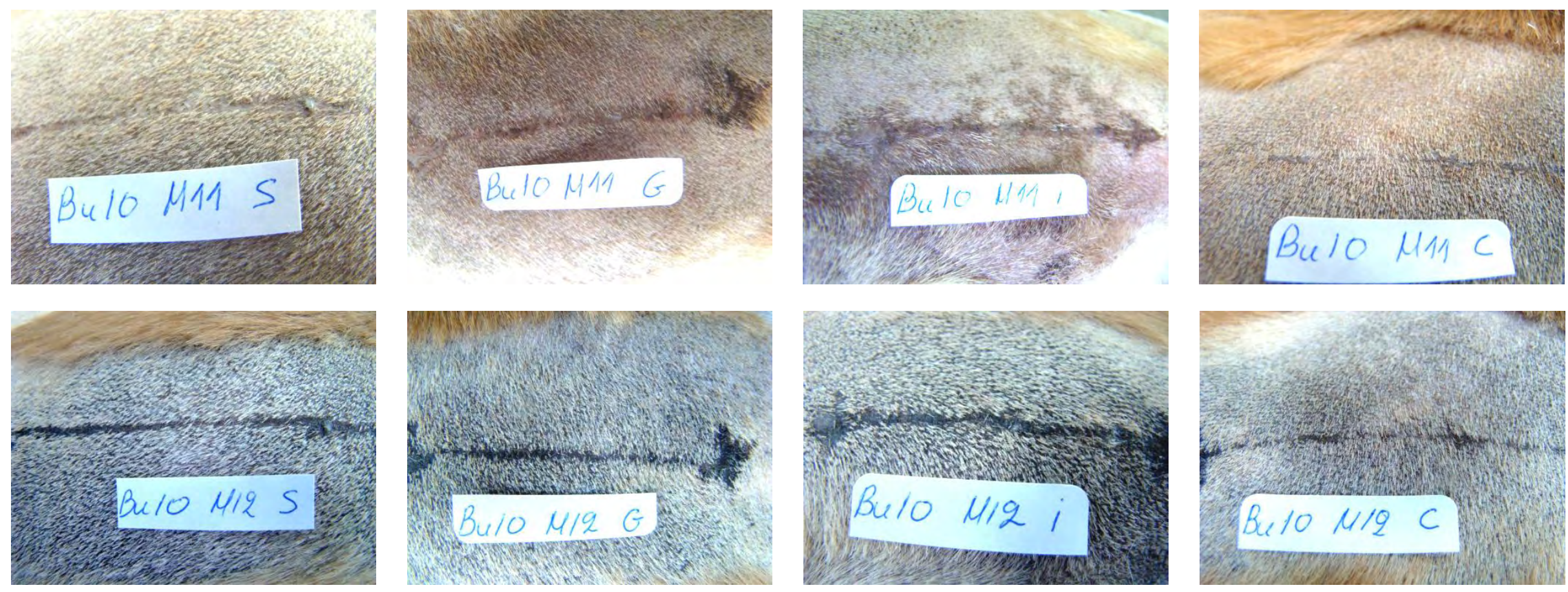


\section{ADDENDUM $\mathbf{l b}$}


Institutional Repository - Library \& Information Centre - University of Thessaly 26/04/2023 13:56:30 EEST - 54.147.182.235 


\section{Day 7}
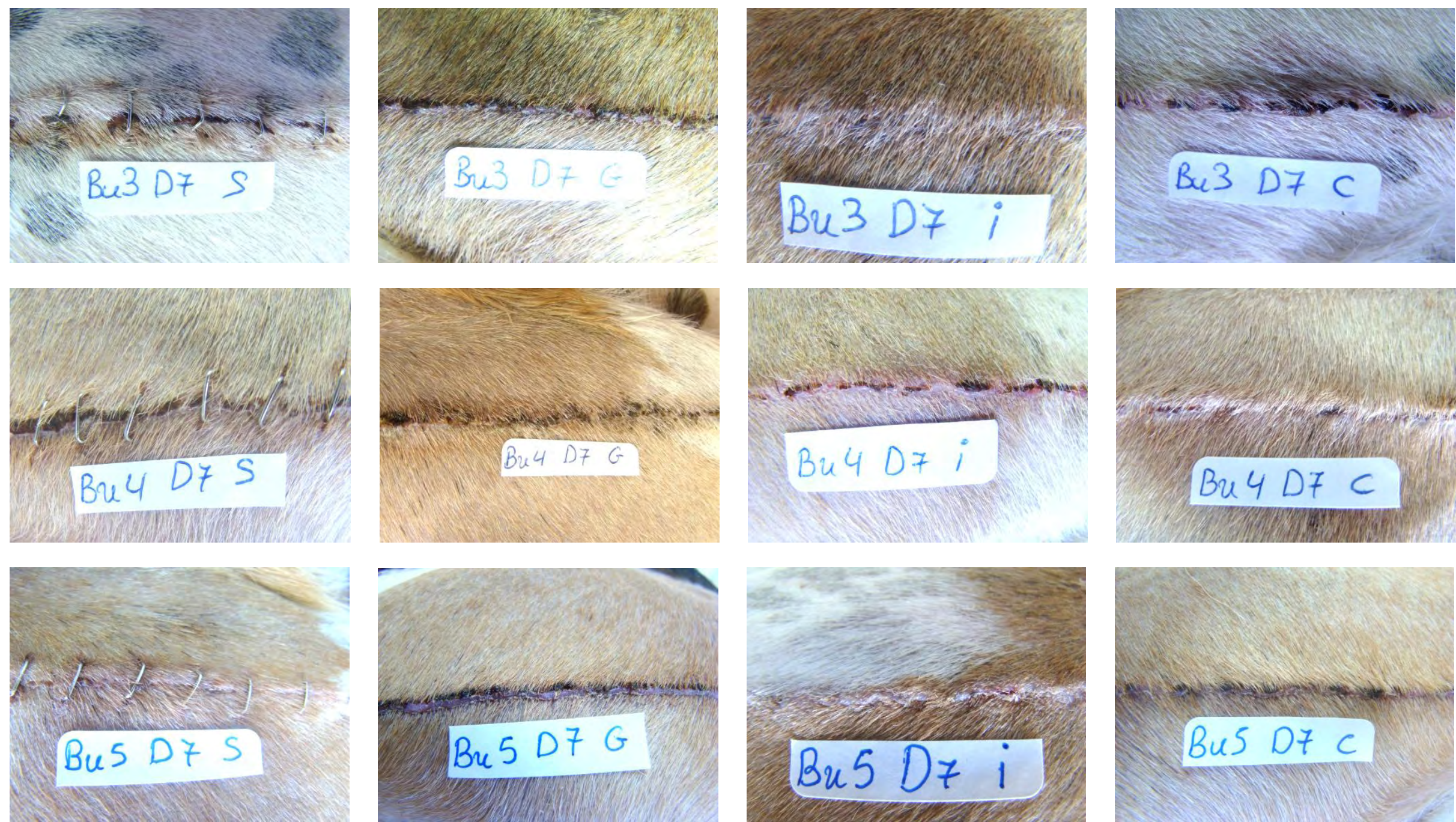

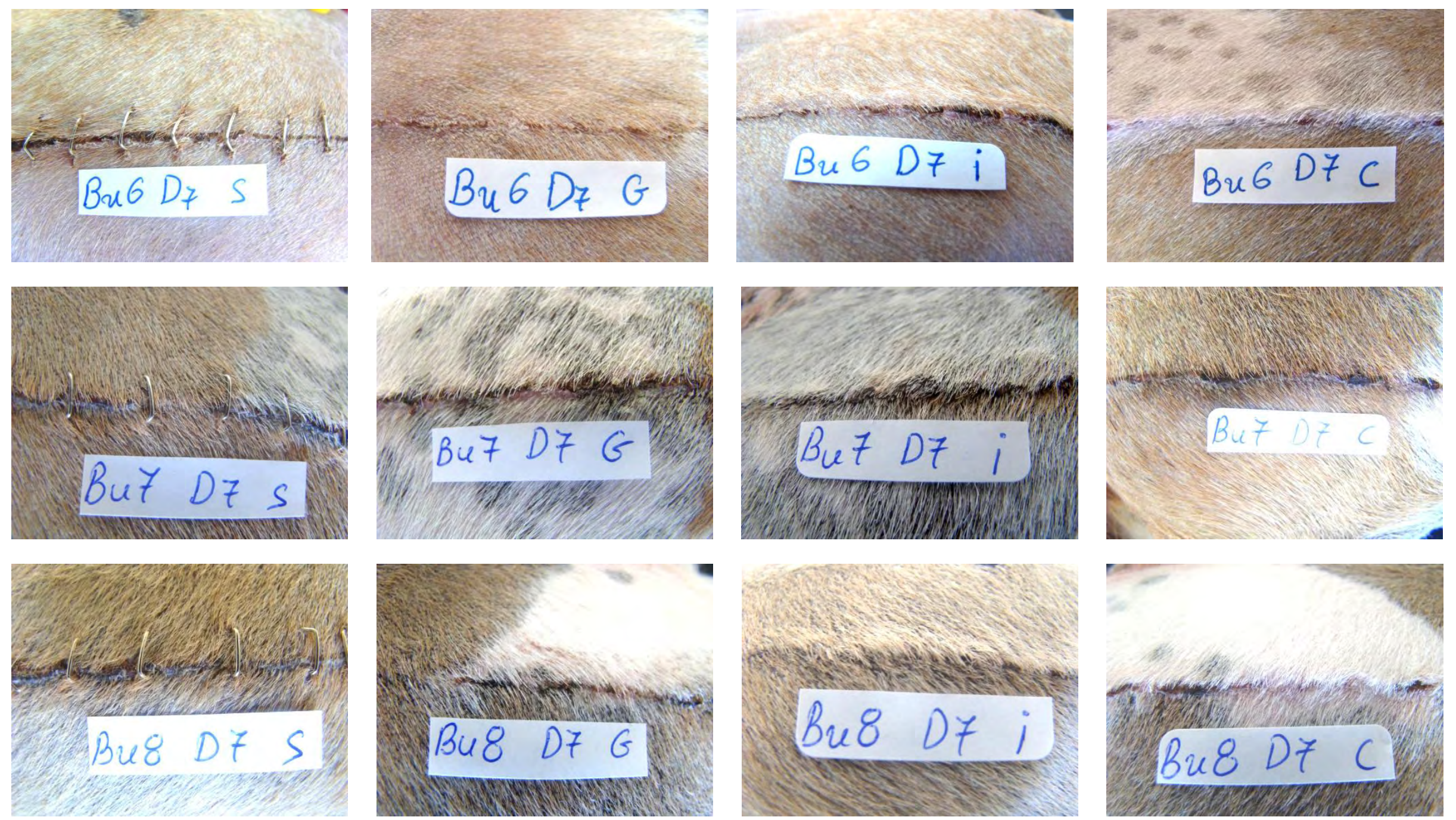

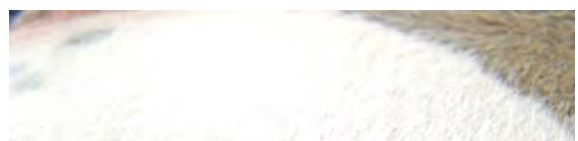

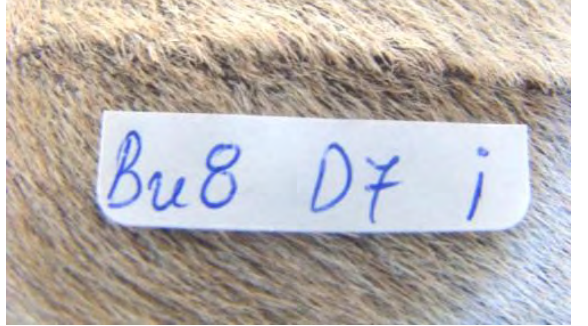



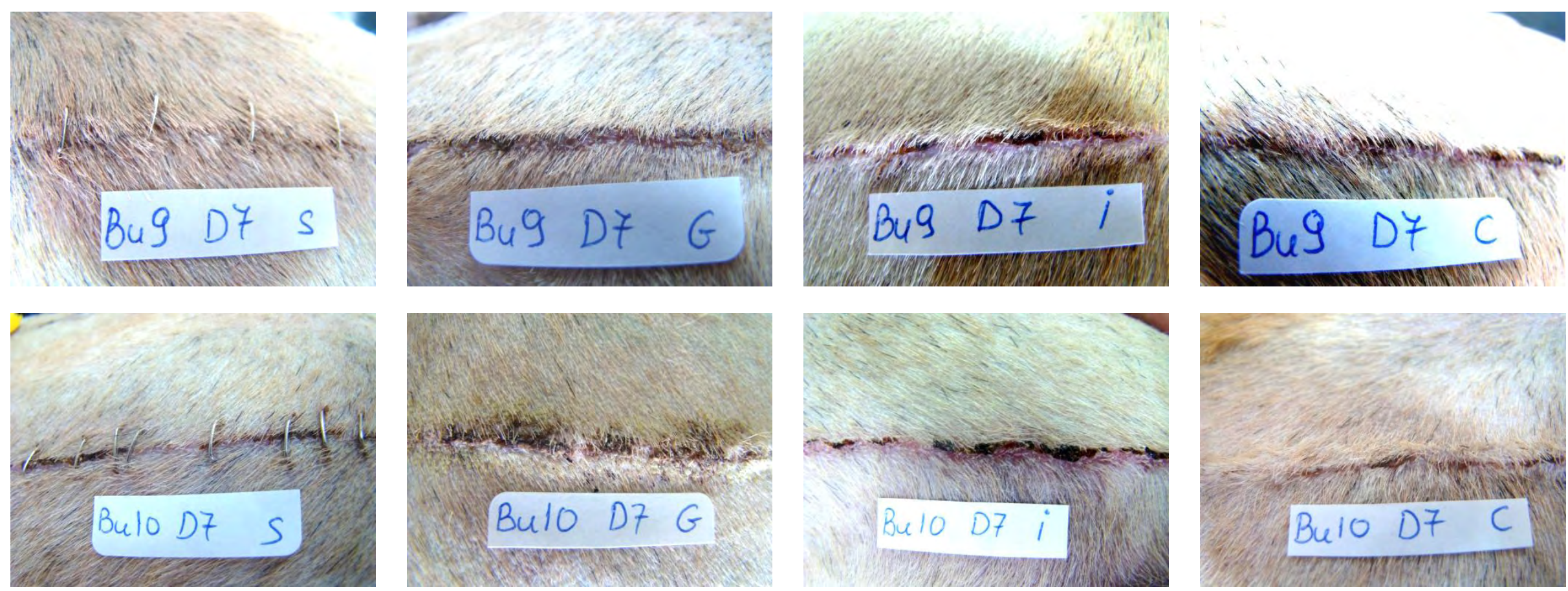


\section{D14}
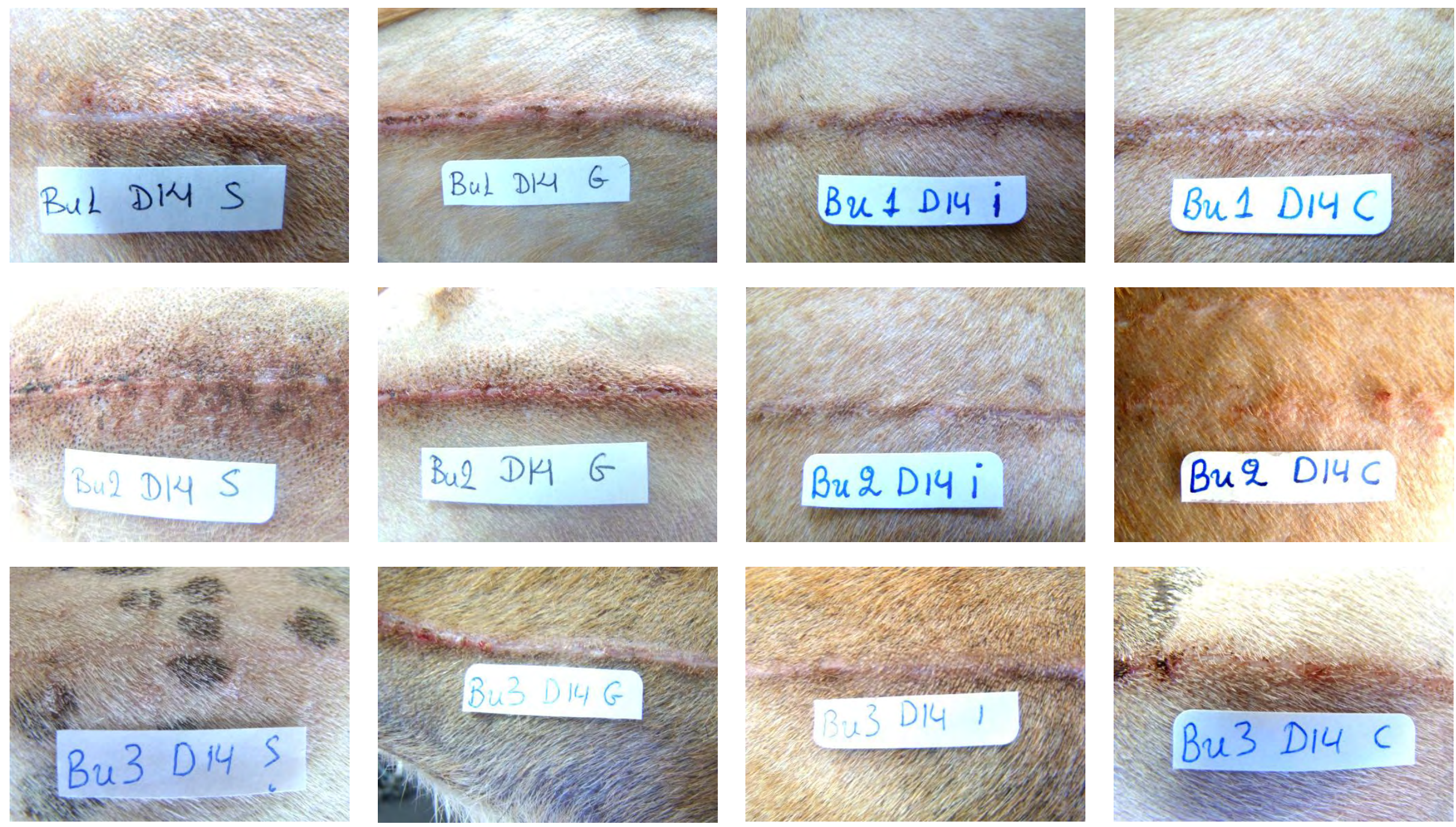

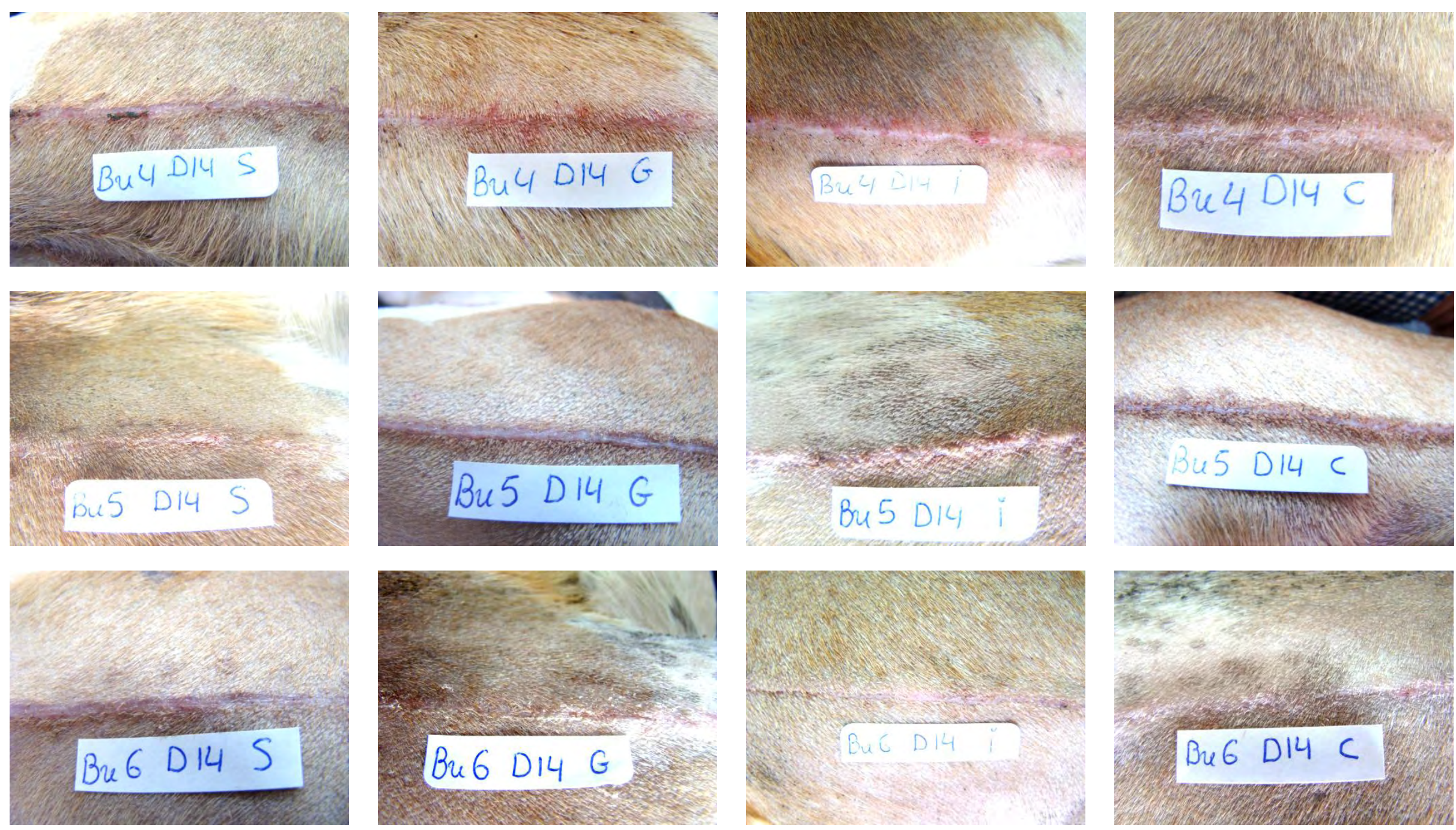

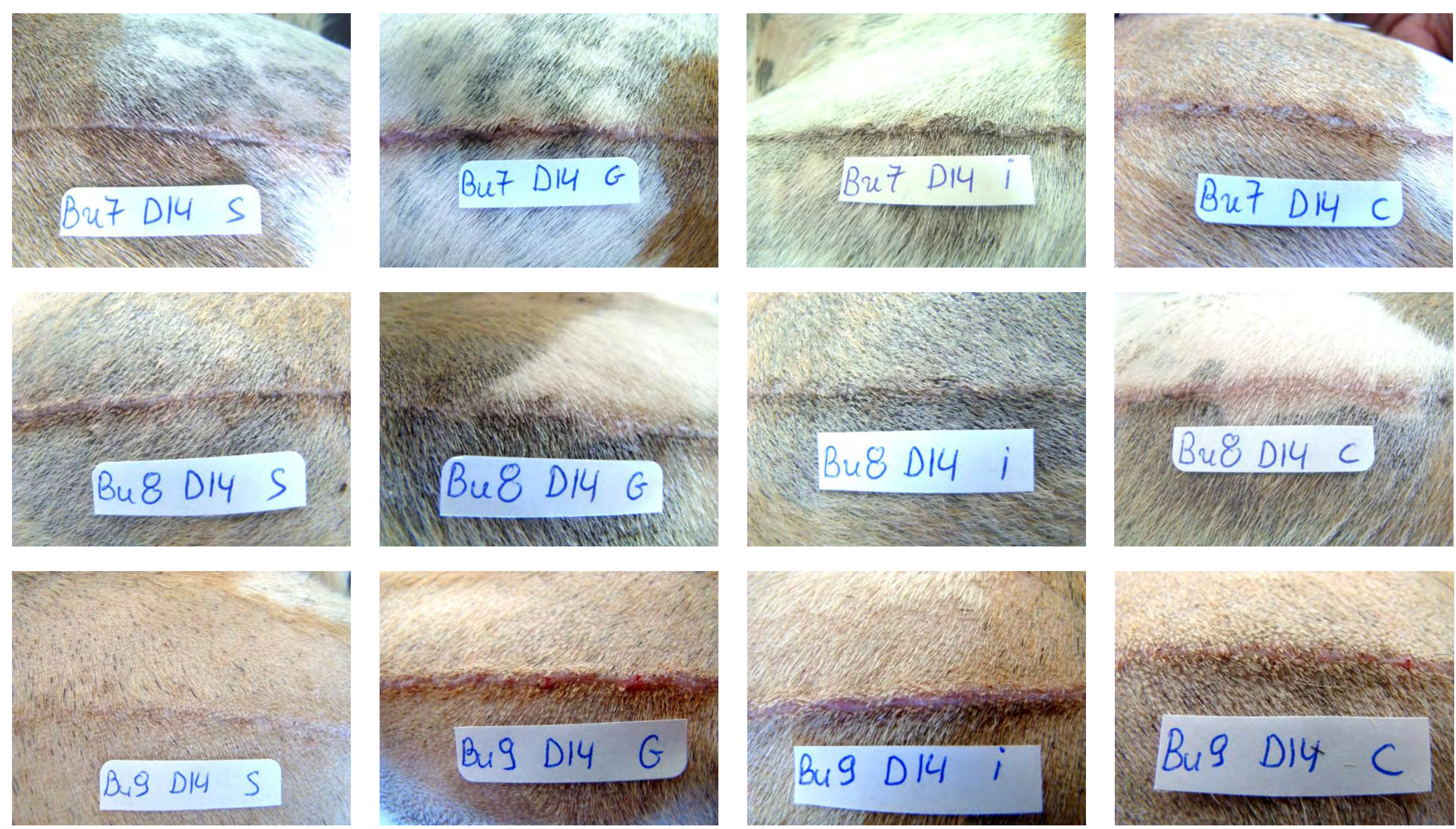

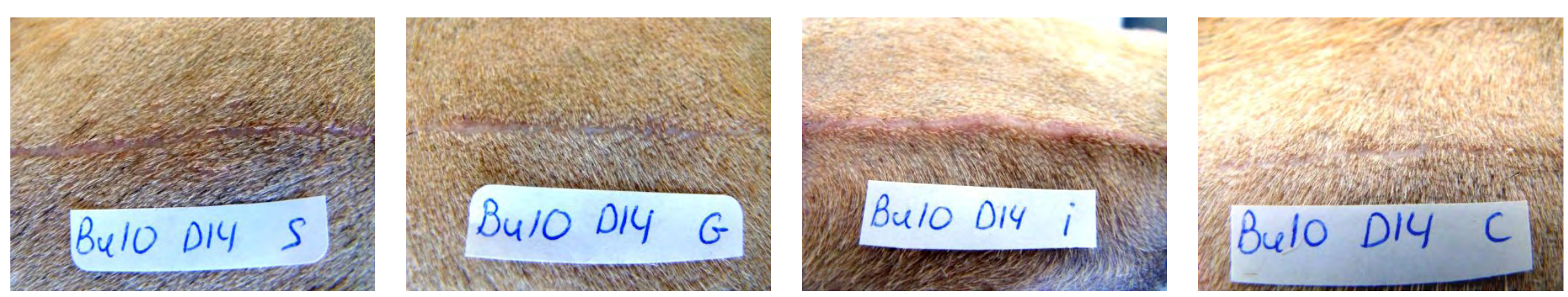


\section{D28}
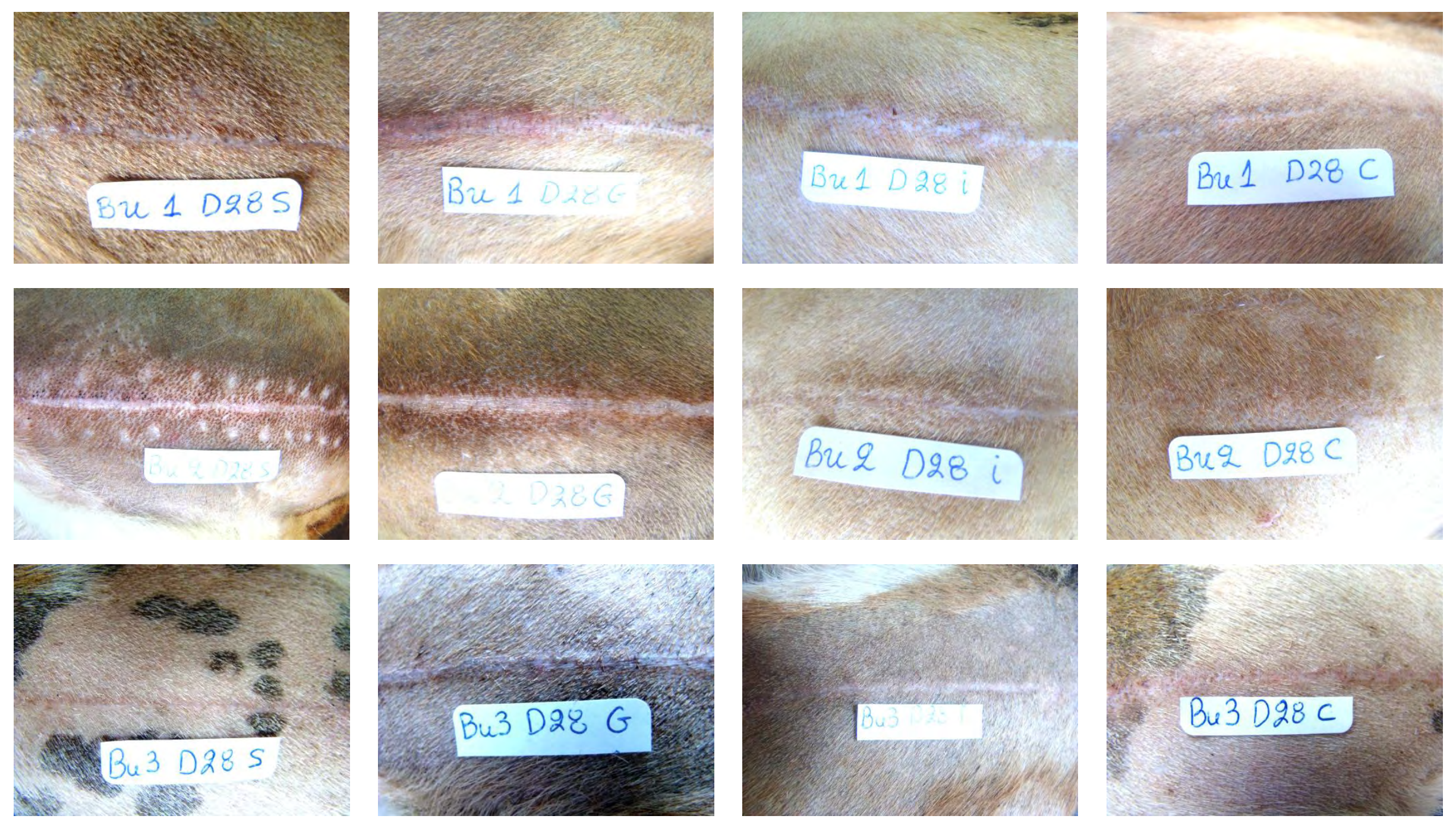

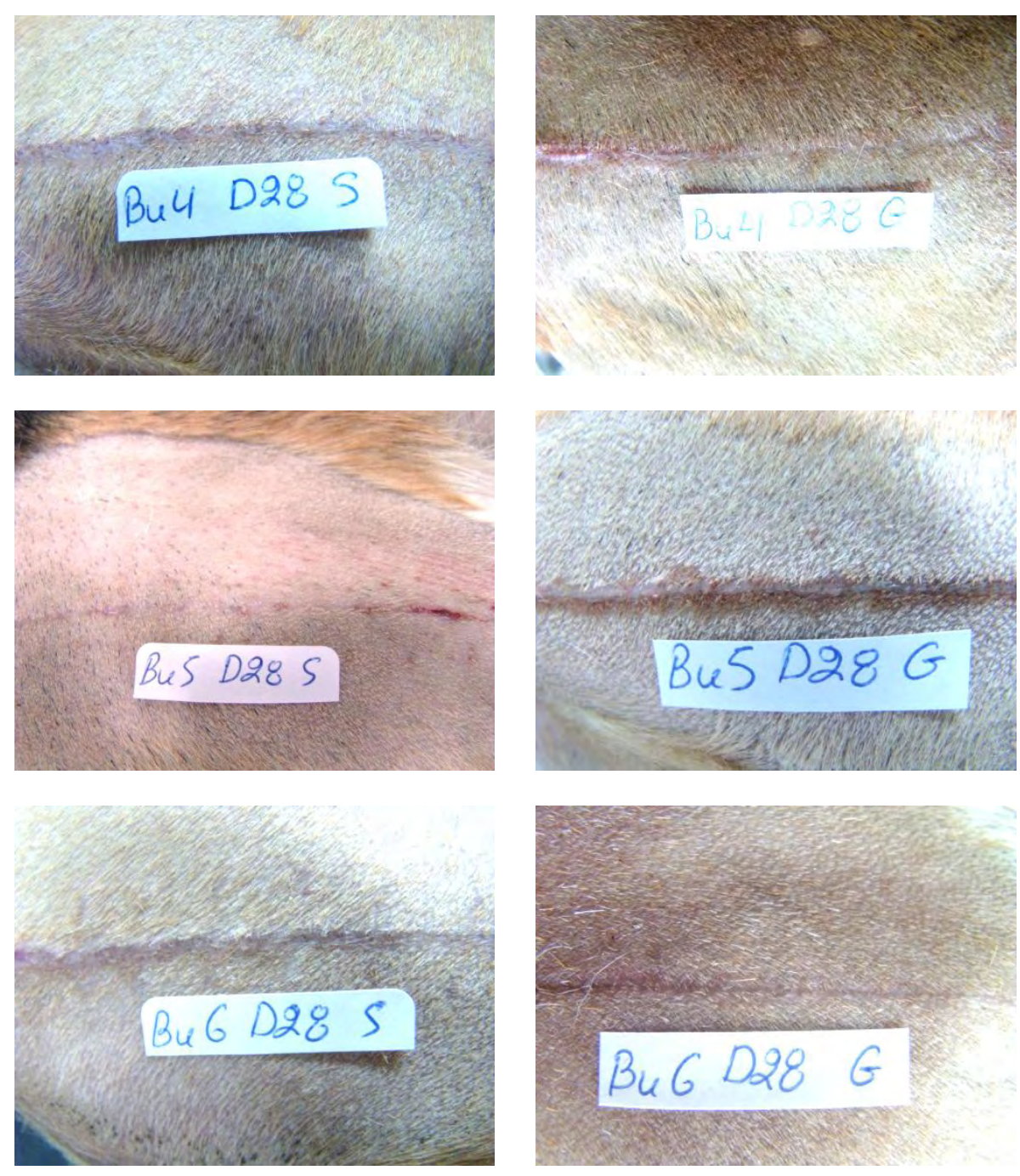
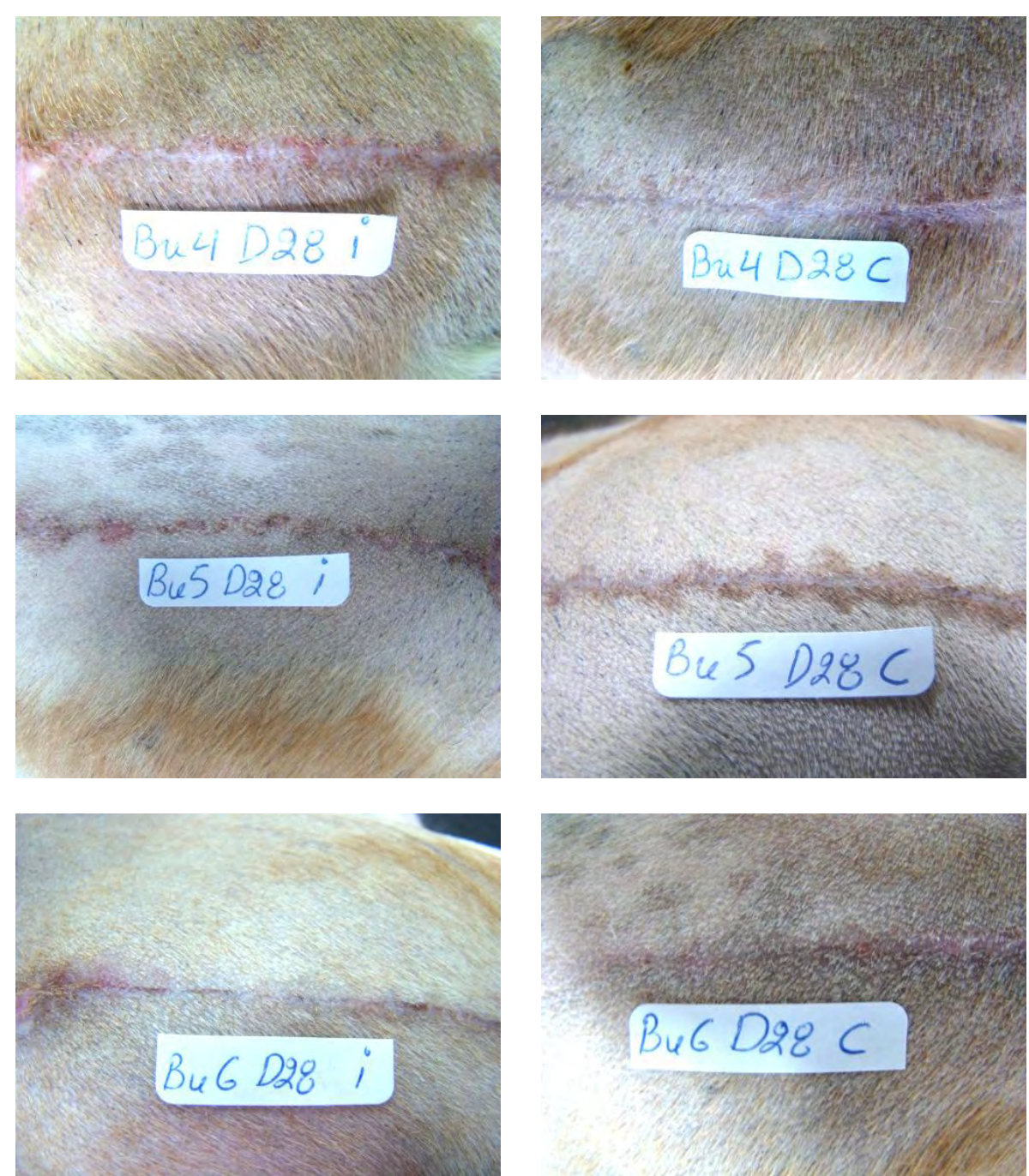

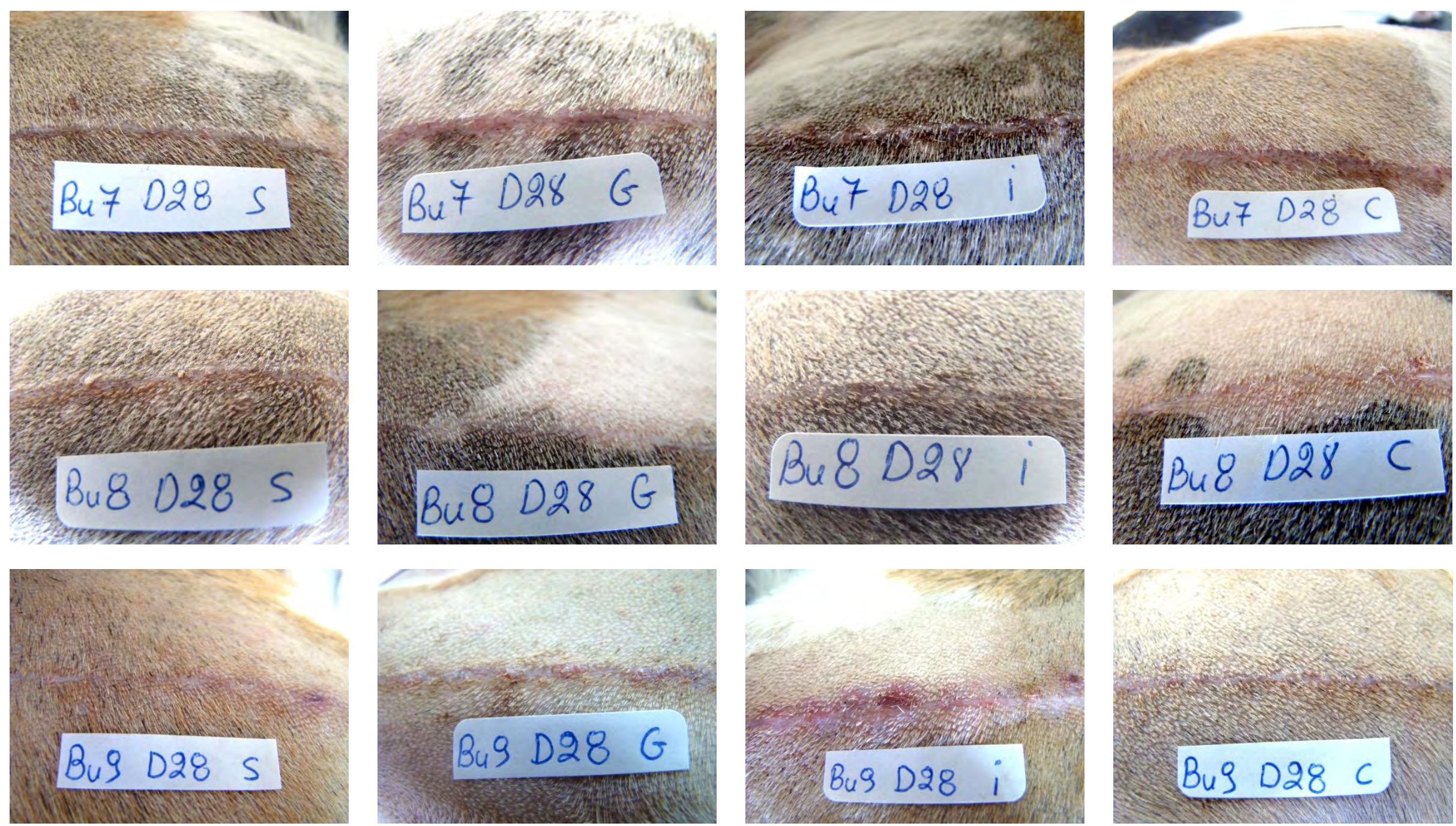

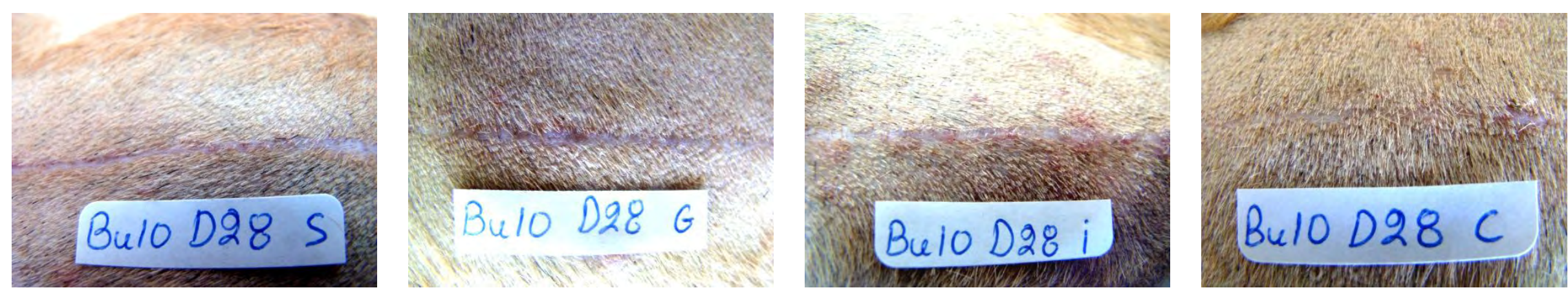


\section{D180}
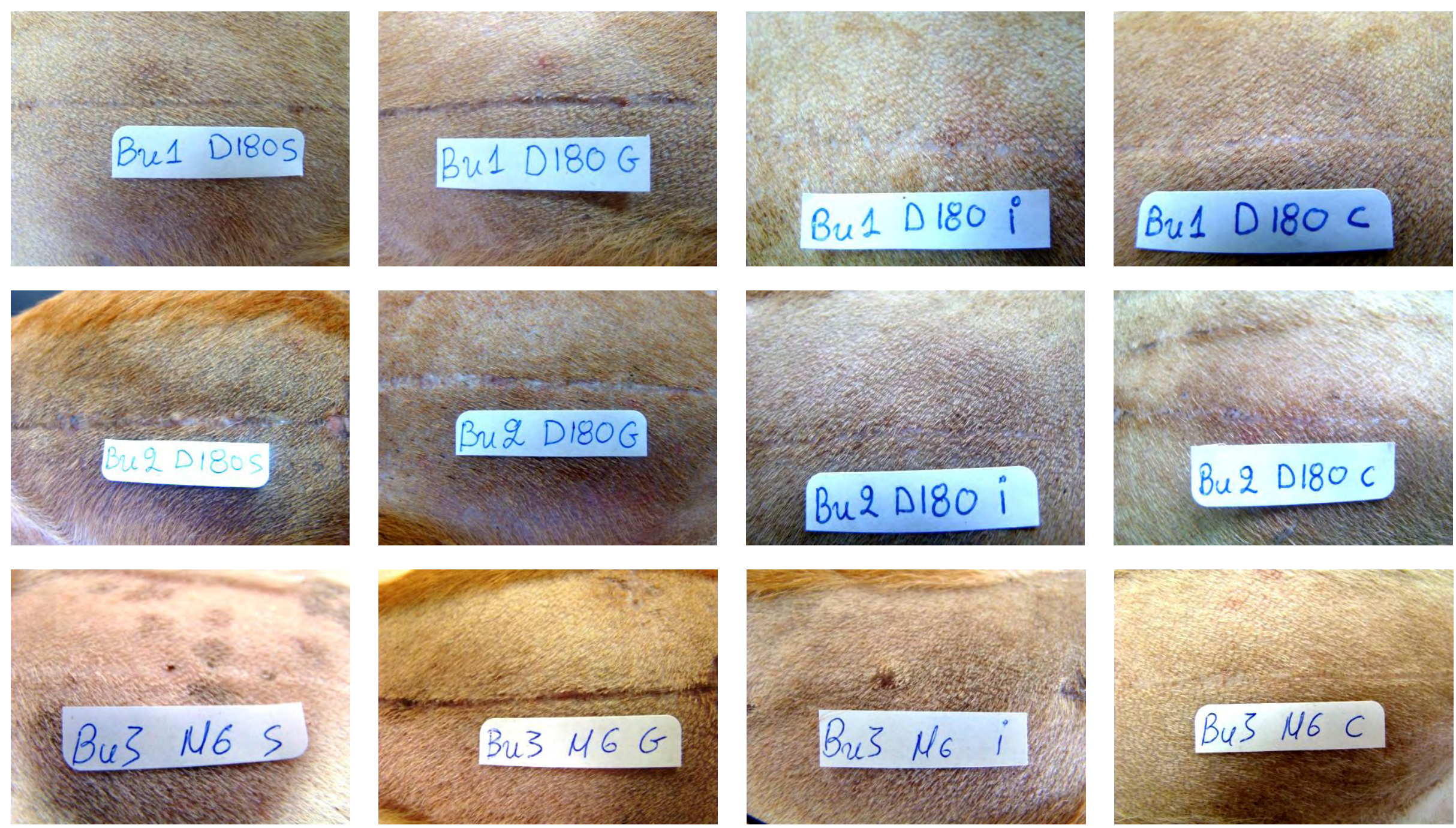

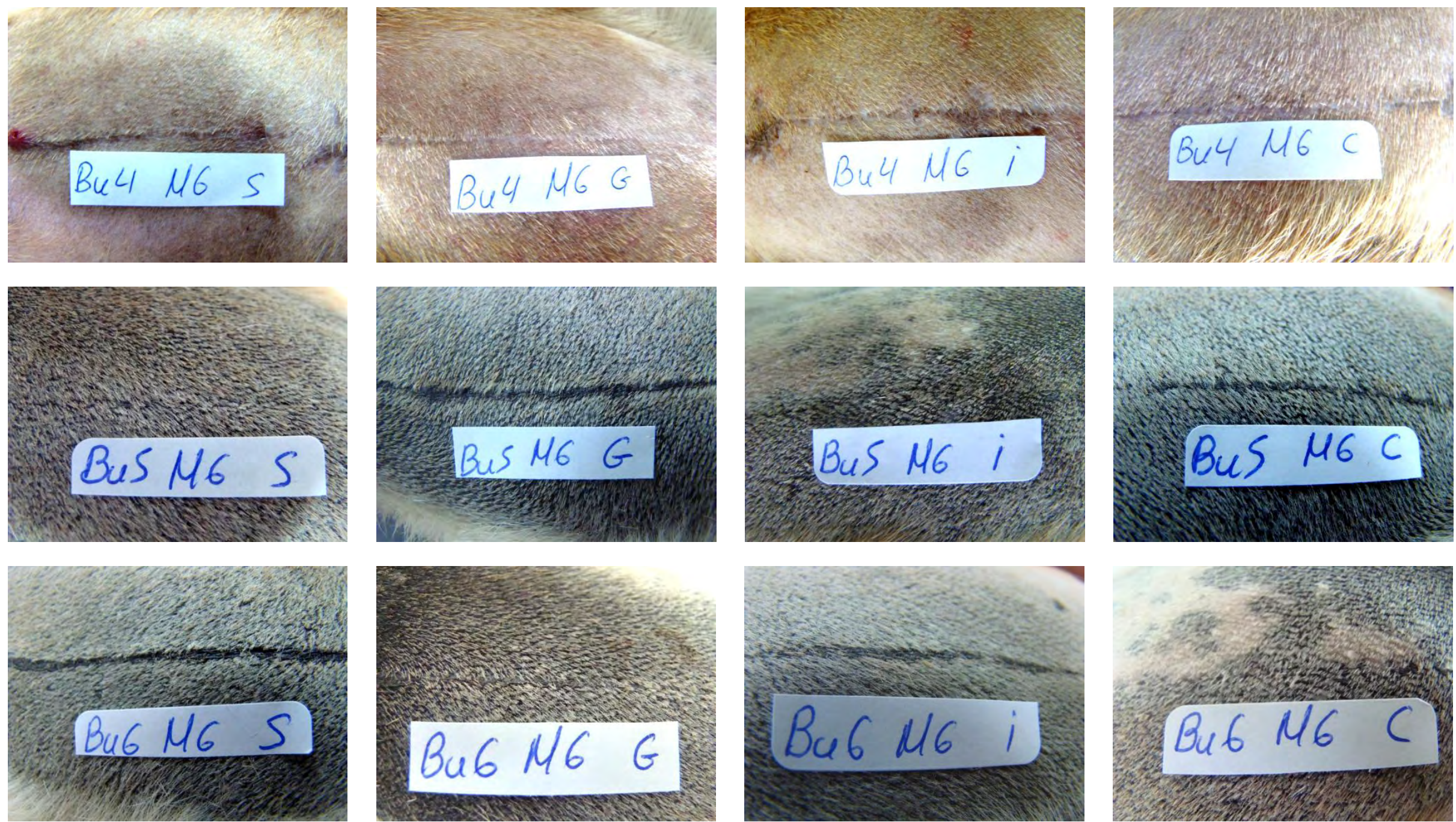

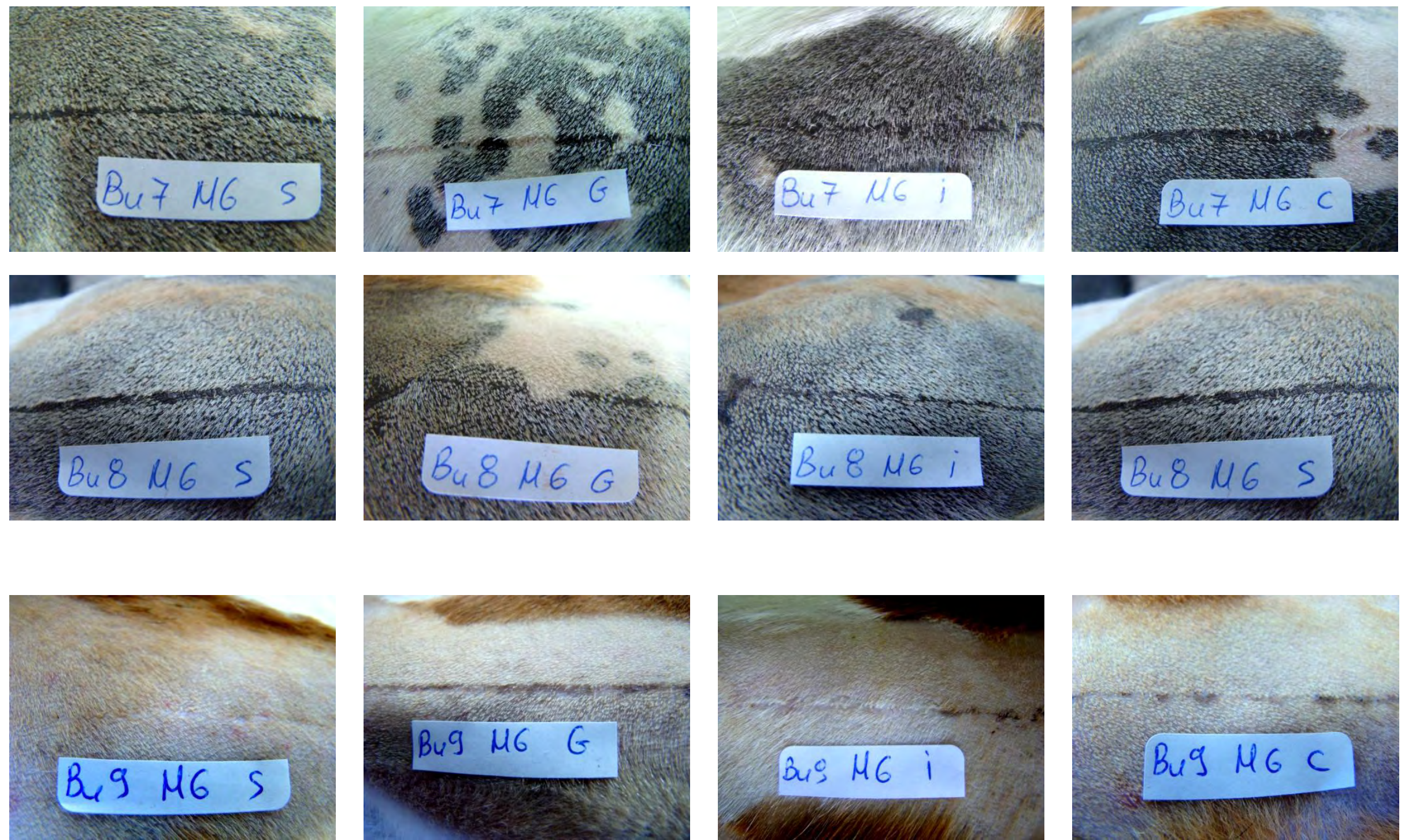

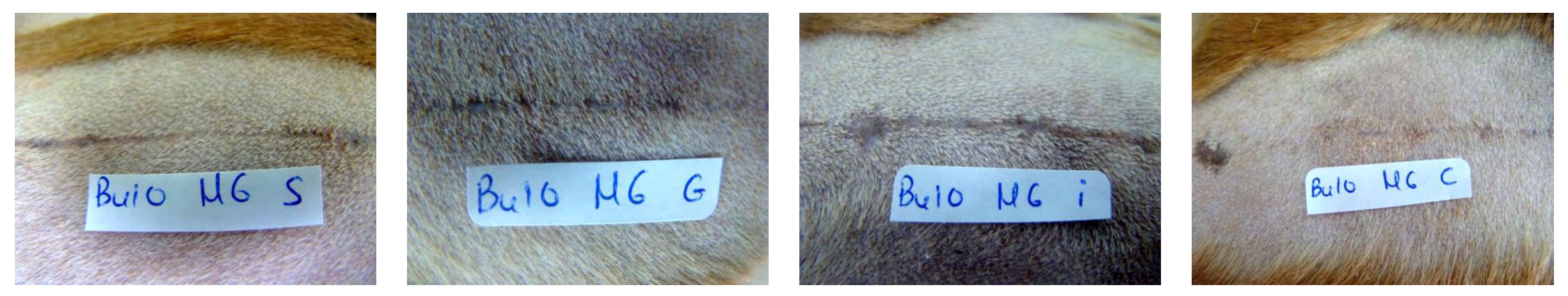


\section{D365}
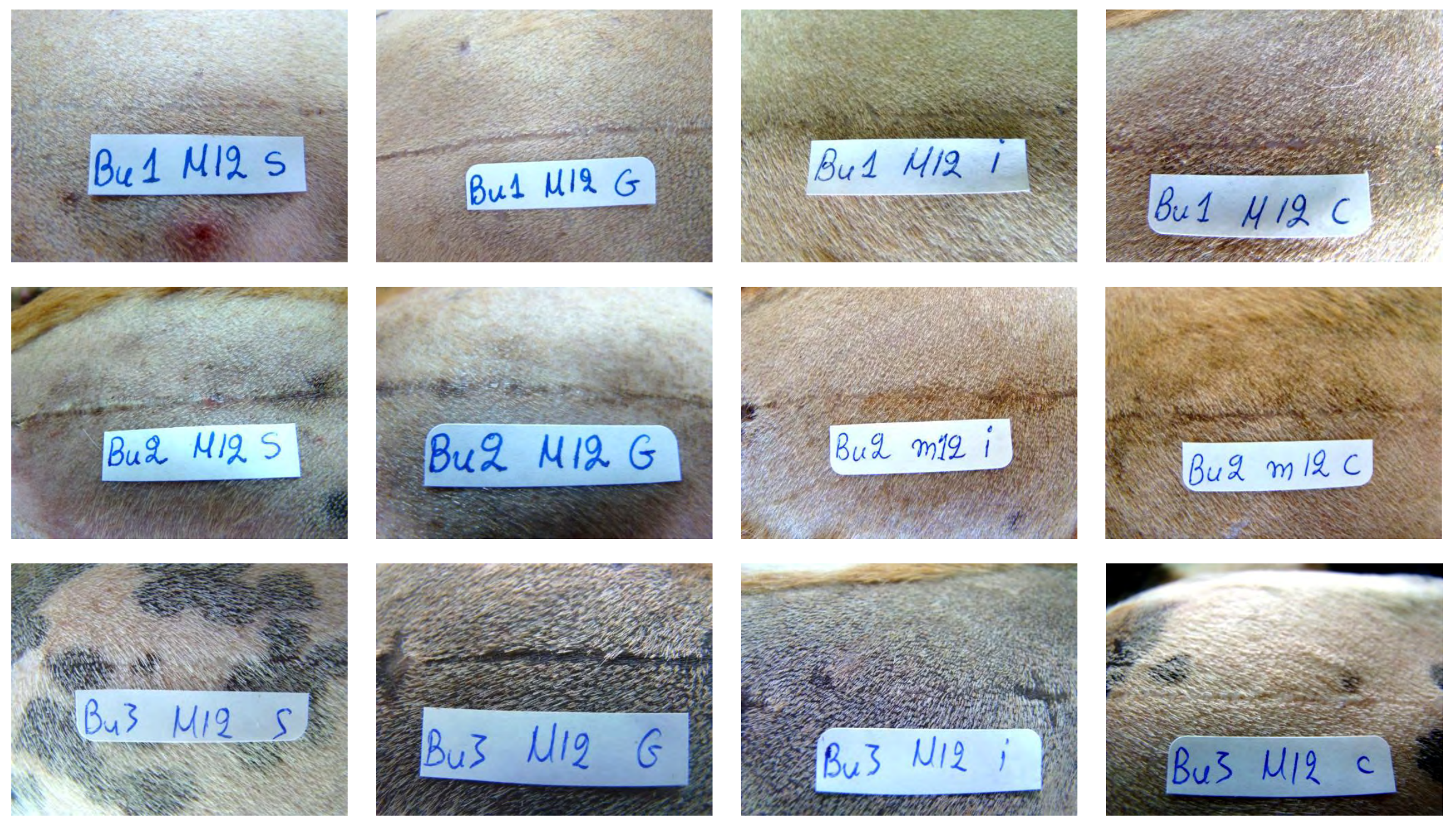

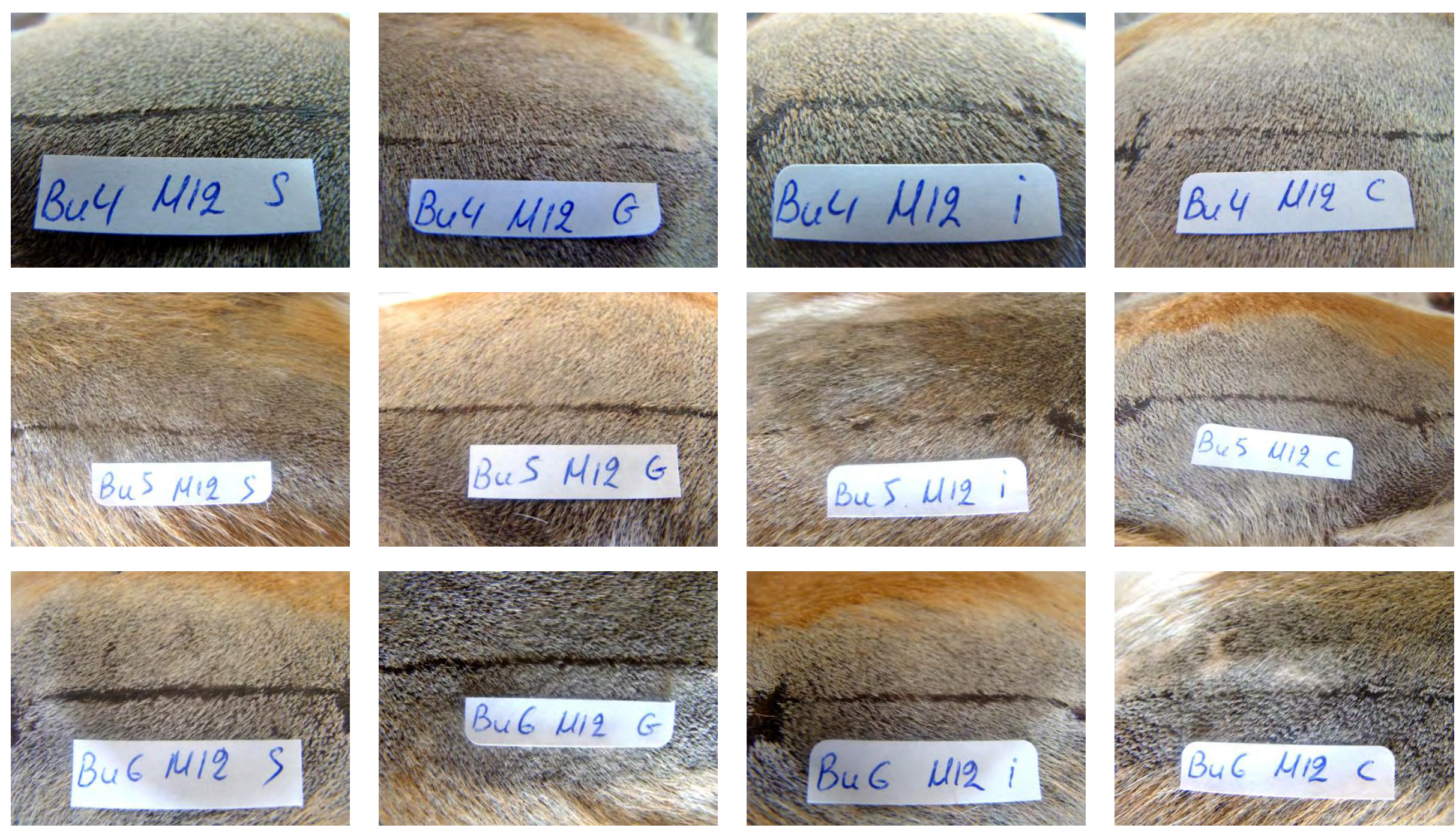

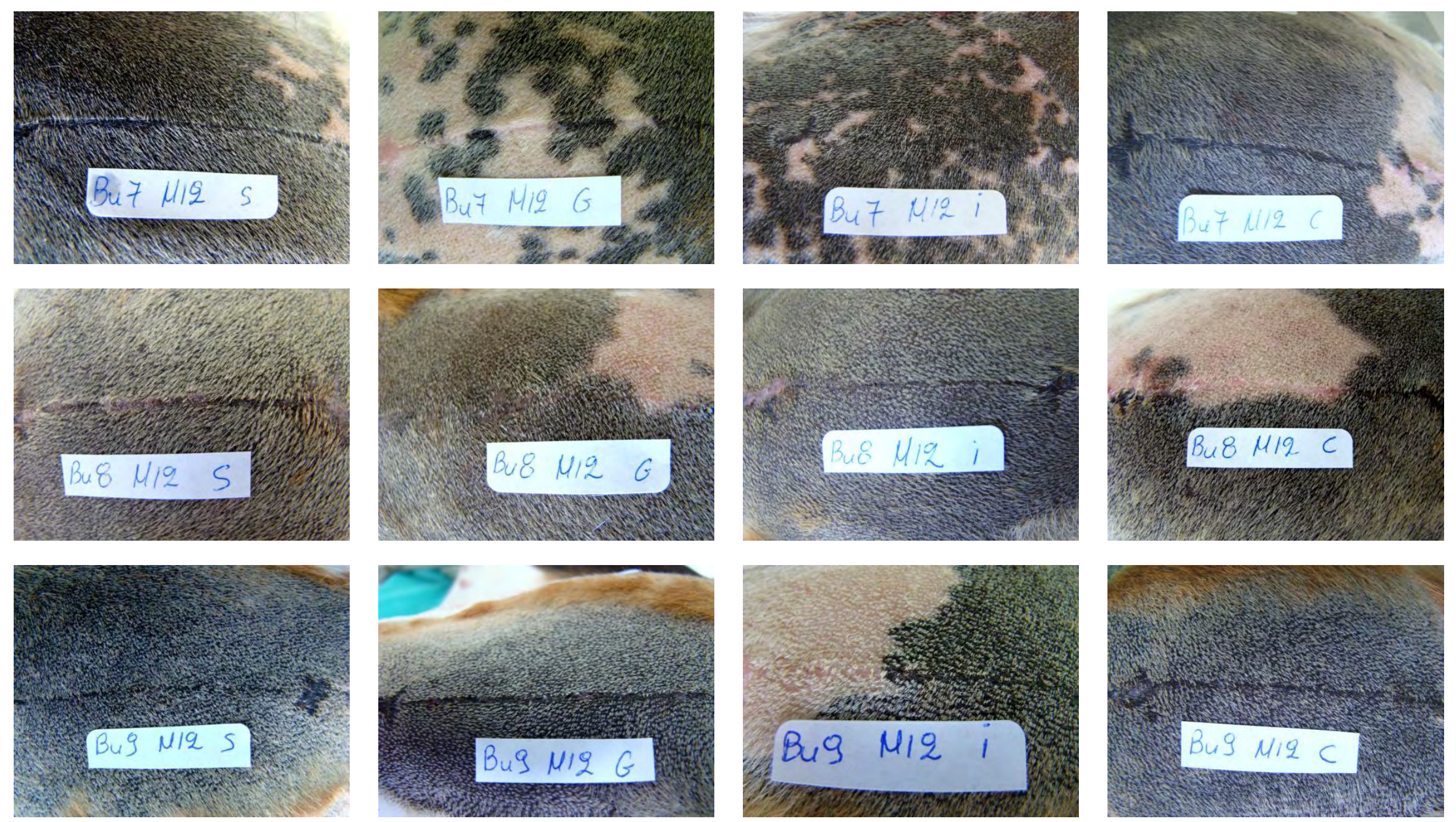

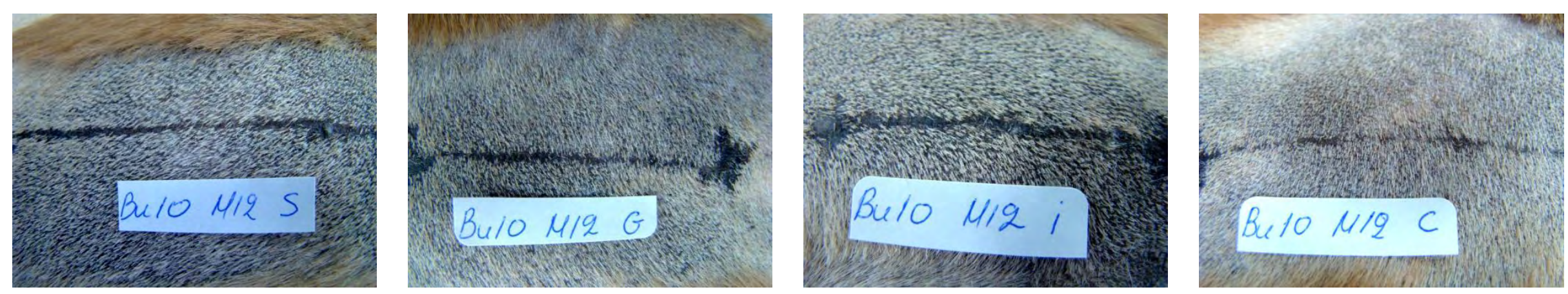
Institutional Repository - Library \& Information Centre - University of Thessaly 26/04/2023 13:56:30 EEST - 54.147.182.235 


\section{ADDENDUM 2}


Institutional Repository - Library \& Information Centre - University of Thessaly 26/04/2023 13:56:30 EEST - 54.147.182.235 
Ultrasonographic scans from Beagle Bu1
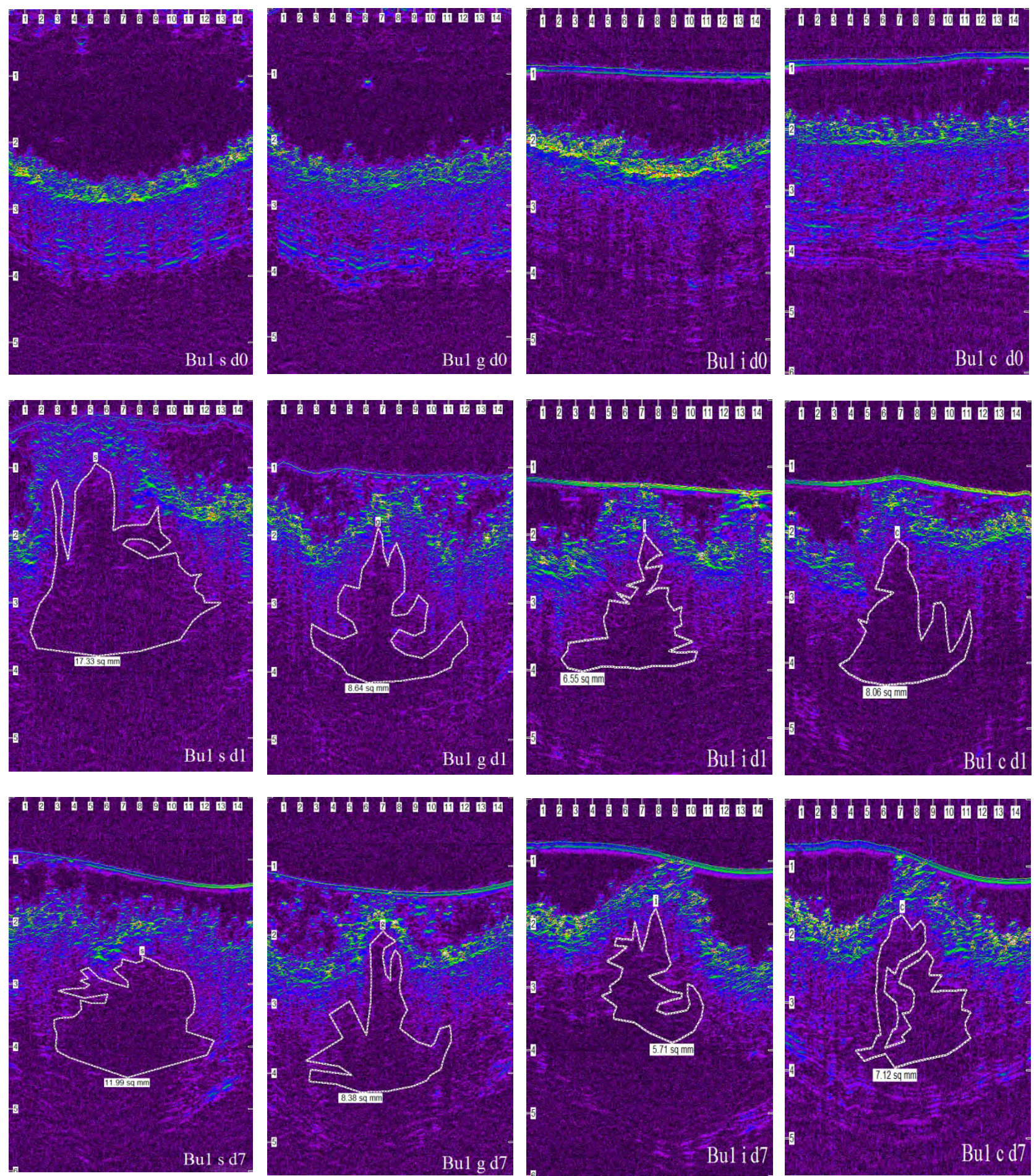

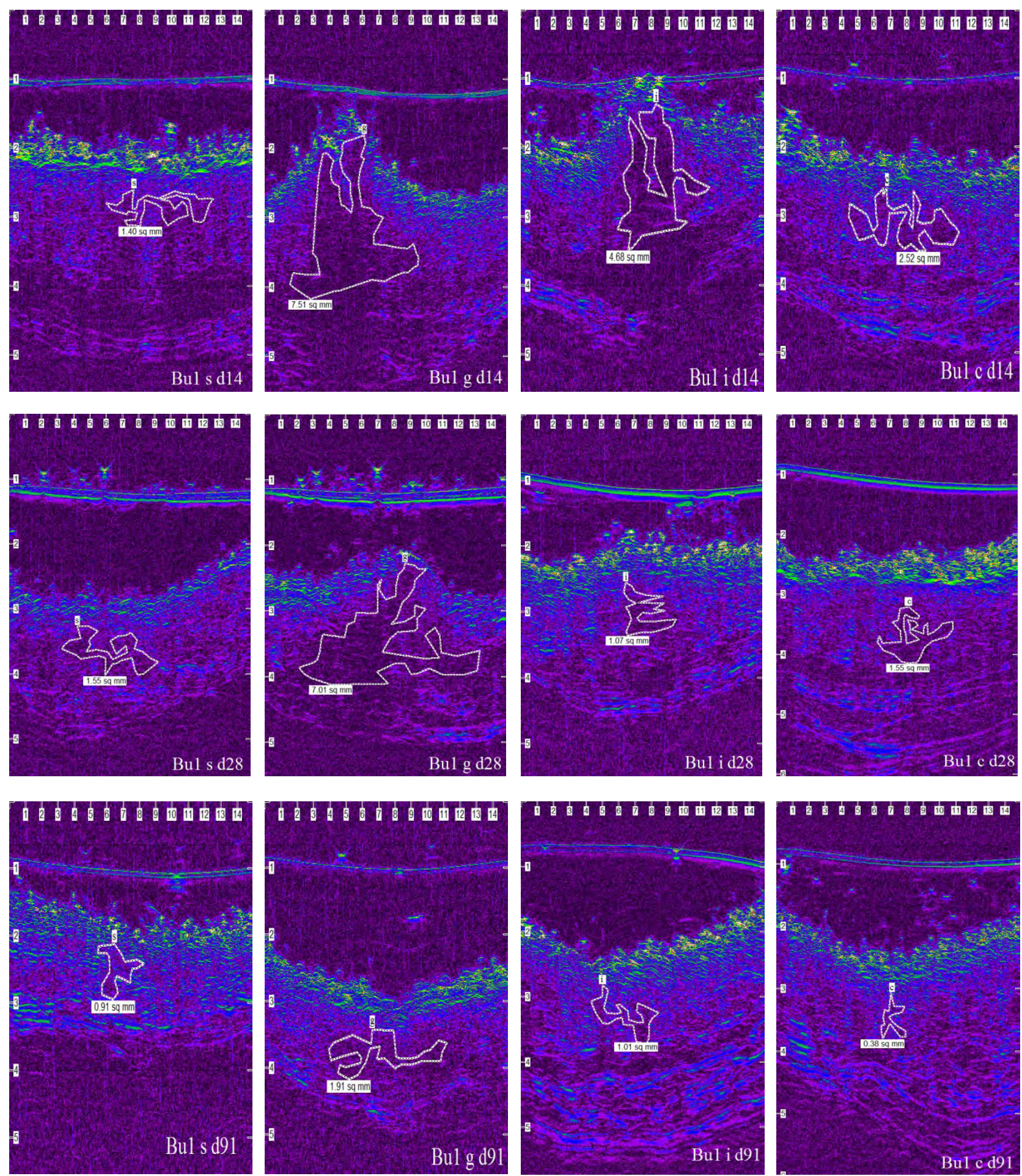

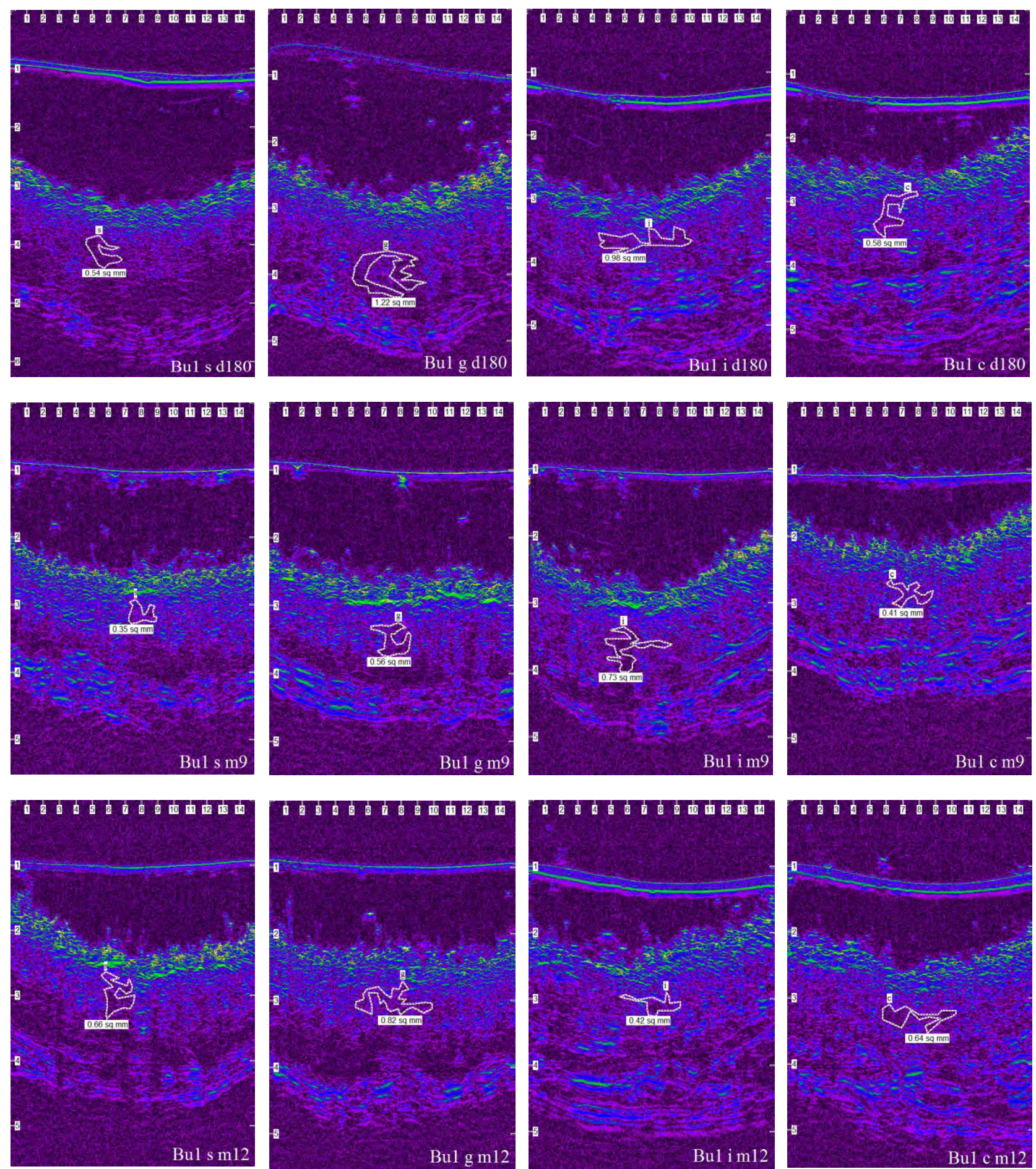